\title{
UTERINE PAPILLARY SEROUS CARCINOMA
}

\section{HANNAH CAMPBELL}

Doctor of Medicine (MD)

The University of Edinburgh 


\section{Declaration of Originality}

This thesis has been composed by me and has neither been presented nor accepted in any previous application for any other degree or professional qualification.

All work presented in this thesis was, unless acknowledged, carried out by myself.

Hannah Campbell 


\section{Table of Contents}

$\begin{array}{ll}\text { Title Page } & 1\end{array}$

Declaration of Originality 2

Table of Contents 3

Acknowledgements 4

Dedication 5

$\begin{array}{ll}\text { Abstract } & 6\end{array}$

Index of Figures $\quad 8$

Index of Tables 11

$\begin{array}{ll}\text { Abbreviations } & 13\end{array}$

$\begin{array}{ll}\text { Chapter } 1 & 15\end{array}$

Chapter 2

$\begin{array}{ll}\text { Chapter } 3 & 58\end{array}$

$\begin{array}{ll}\text { Chapter } 4 & 72\end{array}$

$\begin{array}{ll}\text { Chapter } 5 & 92\end{array}$

$\begin{array}{ll}\text { Chapter } 6 & 113\end{array}$

$\begin{array}{ll}\text { Chapter } 7 & 130\end{array}$

$\begin{array}{ll}\text { Summary of Thesis } & 149\end{array}$

$\begin{array}{ll}\text { References } & 153\end{array}$

Appendix 1 Bound papers arising from study 195

Appendix 2 Primary data for TMA (p53) 196

Appendix 3 Primary data for TMA (ER) 198 


\section{Acknowledgements}

The following people helped me with this work:

Ms Nicola MacWhinnie (BMS), who made the tissue microarrays, cut sections and performed the immunohistochemistry.

Dr A Stillie (Oncology SPR), who reviewed oncology notes for the audit on the pathology and management of uterine papillary serous carcinoma (UPSC) and correlation with outcome (Chapter 4).

Dr D Faratian (Pathology SPR), who helped with the statistical analysis for audit on the pathology and management of uterine papillary serous carcinoma (UPSC) and correlation with outcome (Chapter 4) and contributed to writing the paper that resulted from the study.

Dr A R W Williams (Consultant Histopathologist with a special interest in Gynae pathology) co-reviewed the cases of UPSC and helped identify the areas to be sampled. He also proofread the chapters prior to submission. Most importantly, he acted as my supervisor for the thesis and his continuous support and enthusiasm helped stimulate me to complete the work.

My Parents: John and Maggie Monaghan, who together have shown me that by hard work and dedication, many dreams can be achieved. 


\section{Dedication}

I dedicate this thesis to my husband Stephen, my sons Frederick and David and my daughter Zoe, whose impending birth concentrated my mind. 


\section{Abstract}

Uterine Papillary Serous Carcinoma (UPSC) is an uncommon form of endometrial cancer comprising $10 \%$ of all endometrial carcinomas. It is a highly aggressive tumour and because of its poor prognosis, it accounts for a disproportionately large proportion of deaths from endometrial carcinoma. The reason for its aggressive behaviour is uncertain. The aim of the thesis was to investigate potential mechanisms of invasion of UPSC, using an immunohistochemical approach, using high-grade endometrioid endometrial carcinoma (EEC) as a comparator.

An audit of all cases of UPSC diagnosed in South East of Scotland over a 10 year period confirmed the poor prognosis of UPSC and showed that this was conferred even in those cases where the tumour was composed of 5\% UPSC regardless of the additional tumour type. It also raised awareness of the need for accurate and complete surgical staging. Tissue microarrays (TMAs) were created from the central viable part of the tumour and the invasive edge of UPSC and EEC and were shown to be valid for studying expression of the markers involved in this study. There was intertumoural variation in expression of E- and P-cadherin, CD98, matrix metalloproteases (MMPs) -2 and -9 and ER and PR in both UPSC and EEC. ER status is known to affect expression of E cadherin, $\beta$ catenin, CD98 and MMPs -2 and -9 , and E cadherin levels were decreased and the other protein molecules all showed higher expression in EEC compared to UPSC. The results are consistent with the role that oestrogen plays in the development of EEC.

Intratumoural variation in expression of $\mathrm{E}$ cadherin, $\mathrm{P}$ cadherin, $\beta$ catenin, CD98, Galectin-3, MMPs -2 and -9 was demonstrated, supporting the theory of a subclone of the tumour developing properties necessary for invasion. These data contribute to 
the growing body of literature on UPSC, and address diagnostic and treatment uncertainties for the pathology, surgical and oncological teams.

\section{Ethical Approval}

The Medicine/Oncology II Research Ethics Committee of the Lothian research Ethics Committee gave ethical approval for this study. 


\section{List of Figures}

21A Figure 1.1 Diagram of the cell cycle courtesy of Cotran et al \{Cotran RS, 2006\} Copyright (C) Elsevier 2005.

29A Figure 1.2 Diagram of the Metastatic cascade. \{Cotran RS, 2006\} Copyright (C) Elsevier 2005.

46A Figure 2.1 Papillary Architecture of UPSC.

46A Figure 2.2 Solid Architecture of UPSC.

46B Figure 2.3 Pleomorphic Nuclei and Multinucleation of UPSC.

46B Figure 2.4 Psammoma Bodies of UPSC.

47A Figure 2.5 Classical Gland Formation of EEC.

47A Figure 2.6 Solid Areas of EEC.

47B Figure 2.7 Stratification of Cells of EEC.

47B Figure 2.8 Pleomorphic Nuclei of EEC.

47C Figure 2.9 Circle Marking Site of Core on Whole Section.

49A Figure 2.10 Beecher Instrument courtesy of Skacel et al \{Skacel, 2002\}.

49A Figure 2.11 Tissue Microarray Block.

63A Figure 3.1 Expression of P53 in UPSC Tissue Microarray.

63A Figure 3.2 Expression of ER in UPSC Tissue Microarray.

66A Figure 3.3 Whole Section showing P53 with hole at site of Core Biopsy.

66A Figure 3.4 Whole Section showing ER with hole at site of Core Biopsies.

69A Figure 3.5 Whole Section showing Expression of P53 in Stage 1a UPSC. 
69A Figure 3.6 Whole Section showing Expression of P53 in infiltrating UPSC.

79 Figure 4.1 Overall Survival and Progression-free Survival by Stage.

81 Figure 4.2 Kaplan-Meier curves of Treatment Variables by Overall Survival.

83 Figure 4.3 Percentages of Histological Subtypes in Diagnostic Biopsies Compared to Resection Specimens.

84 Figure 4.4 Overall Survival according to Percentage of UPSC.

104A Figure 5.1 E Cadherin Expression in UPSC.

104A Figure 5.2 P Cadherin Expression in UPSC.

104B Figure $5.3 \beta$ Catenin Expression in UPSC.

105A Figure 5.4 E Cadherin Expression in Normal Endometrial Epithelium.

105A Figure 5.5 E Cadherin Expression in EEC.

105B Figure 5.6 P Cadherin Expression in EEC.

105B Figure 5.7 Nuclear $\beta$ Catenin Expression in EEC.

122A Figure 6.1 Expression of CD98 in EEC.

122A Figure 6.2 Expression of CD98 in UPSC.

122B Figure 6.3 Expression of Gal-3 in EEC.

122B Figure 6.4 Expression of Gal-3 in UPSC.

142A Figure 7.1 Expression of MMP-2 in UPSC.

142A Figure 7.2 Expression of MMP-7 in UPSC. 
142B Figure 7.3 Expression of MMP-9 in UPSC.

144A Figure 7.4 Expression of MMP-2 in EEC.

144A Figure 7.5 Expression of MMP-7 in EEC.

144B Figure 7.6 Expression of MMP-9 in EEC. 


\section{Index of Tables}

44 Table 2.1. FIGO Staging for Carcinoma of the Endometrium.

45 Table 2.2. Demographic Details for UPSC and EEC patients used to create Tissue MicroArrays.

48 Table 2.3. Grading of Endometrial Carcinoma.

51 Table 2.4. Primary Antibodies and Antigen Retrieval Conditions used in Immunohistochemistry.

64 Table 3.1. Technical Results of TMA stained with H\&E, P53 and ER.

67 Table 3.2. Expression of P53 and ER in TMA and Whole Sections.

78 Table 4.1. Progression Free Survival and Overall Survival by Stage

85 Table 4.2. Chemotherapy Regimens by Stage.

103 Table 5.1. Expression of E Cadherin, P Cadherin and $\beta$ Catenin in the Central Part of the Tumour in UPSC and EEC.

104 Table 5.2. Expression of E Cadherin, $\mathrm{P}$ Cadherin and $\beta$ Catenin in the Central Part of the Tumour and at the Invasive Edge.

122 Table 6.1. Expression of Galectin-3 and CD98 in the Viable Central Part of the Tumour in UPSC and EEC.

124 Table 6.2. Expression of Galectin-3 and CD98 in the Central Part of the Tumour and at the Invasive Edge. 
139 Table 7.1. Expression of MMPs-2, -7 and -9 in the Viable Central Part of the Tumour in UPSC and EEC.

141 Table 7.2. Expression of MMPs-2, -7 and -9 in the Viable Central Part of the Tumour and at the Invasive Edge.

142 Table 7.3. Variation in Matrix Metalloproteinase Expression Between Tumour and Stromal cells in UPSC.

144 Table 7.4. Variation in Matrix Metalloproteinase Expression Between Tumour and Stromal cells in EEC. 


\section{Abbreviations}

List of the main abbreviations used in the text:

UPSC Uterine Papillary Serous Carcinoma

EEC Endometrioid Endometrial Carcinoma

TMA Tissue MicroArray

MMP Matrix Metalloprotease

ECIS Endometrial Carcinoma in Situ

EmGD Endometrial Glandular Dysplasia

ER

Estrogen Receptor

PR

Progesterone Receptor

EGFRF

Epidermal Growth Factor Receptor Family

EGFR

Epidermal Growth Factor Receptor

PDGFR Platelet Derived Growth Factor Receptor

KIP Kinase Inhibitor Protein

Cdks

Cyclin Dependent Kinases

E2F

Elongation Factor 2

$\mathrm{Rb}$

Retinoblastoma Protein

TGF- $\beta$

Transforming Growth Factor- $\beta$

$\mathrm{LOH}$

Loss of Heterozygosity

PTEN

Phosphatase and tensin homologue deleted on chromosome 10

OSPC

Ovarian Serous Papillary Carcinoma

ECM

Extracellular Matrix

TIMP

Tissue Inhibitor of MMP 
FIGO International Federation of Gynaecology and Obstetrics

WART Whole Abdominal Radiotherapy

IL-6 Interleukin-6

EDTA Ethylenediamine Tetraacetic Acid

IHC Immunohistochemistry

FISH Fluorescent In Situ Hybridisation

PFS Progression Free Survival

OS Overall Survival

CCEC Clear cell Carcinoma

TAH Total Abdominal hysterectomy

BSO Bilateral Salpingooopherectomy

APC Adenomatous Polyposis Coli

LAT L type Amino Acid Transporter

GAL Galectin 


\section{Chapter 1}

\section{Literature Review of Uterine Papillary Serous Carcinoma.}

\section{Introduction}

This chapter is a literature review of Uterine Papillary Serous Carcinoma (UPSC). Over the past few years there has been growing awareness of the importance of the diagnosis and consequently there has been an increase in the number of studies on UPSC, ranging from audits on presentation of UPSC through molecular studies, methods of diagnosis and numerous different potential treatment modalities.

\subsection{Incidence and Age}

Endometrial carcinoma is the most common pelvic genital malignancy in the western world \{Creasman, 2003\}. The majority of these cancers are of adenocarcinoma type, arising from endometrial epithelium. The most common subtype is endometrioid endometrial carcinoma (EEC), which accounts for about $80 \%$ of endometrial carcinomas \{Burton, 1998; Santin, 2002; Santin, 2003\}. UPSC is a highly aggressive variant of endometrial carcinoma and accounts for approximately $10 \%$ of cases \{Burton, 1998\}, but a much smaller proportion of Stage I endometrial carcinomas \{Hendrickson, 1983;Bancher-Todesca, 1998;Dembo, 1985\}. Patients present later than those with EEC, with a median age of diagnosis of 65 years for UPSC and 60 years for EEC. 


\subsection{Aetiology}

Endometrial carcinomas are often split into 2 groups, determined by their clinical, histopathological and molecular features \{Bokhman, 1983;Sherman, 1992\}. These groups comprise: EEC (Type I) and non-endometrioid endometrial carcinomas (Type II). EEC usually arises from atypical hyperplasia of the endometrium of premenopausal, perimenopausal and postmenopausal women and is associated with nulliparity, obesity, exogenous oestrogen therapy and tamoxifen therapy. In contrast, UPSC usually occurs in elderly, postmenopausal women and is not associated with either atypical hyperplasia or oestrogen status. Indeed, most tumours arise from atrophic endometrium from a precursor lesion, Endometrial Carcinoma in Situ (ECIS), also known as Endometrial Intraepithelial Carcinoma, or Uterine Surface Carcinoma \{Moll, 1996;Zheng, 1998;Spiegel, 1995;Sherman, 1992\}. Zheng et al \{Zheng, 2004\} recently postulated that UPSC and ECIS might develop from dysplastic endometrial glands rather than de novo from normal postmenopausal endometrium. In a small series of cases they found endometrial glandular dysplasia (EmGD) in 17/32 (53\%) uteri with UPSC compared with 1/60 (1.7\%) uteri removed for EEC. EmGD was identified in 12/16 (75\%) ECIS uteri. Areas of both EmGD and ECIS were found in 15/32 (47\%) of the UPSC uteri. Transitions from either EmGD to ECIS or ECIS to UPSC were present in $8 / 32$ (25\%) of the UPSC cases. No transitions from EmGD to UPSC were identified in any hysterectomy specimen. EmGD was frequently found in endometrial polyps. There was no statistically significant difference between EmGD in a polyp (48\%) and EmGD in non-polypoid endometrium (52\%). In the majority of cases, EmGD was multifocal and involved superficial endometrial glands. However, single glandular involvement and surface 
epithelial involvement of the endometrium was also seen. Although this is a small series, it does seem likely that there is some type of "interval" stage from normal endometrium to ECIS. However, further study is required in this area to elucidate the molecular changes occurring at these different stages.

\subsubsection{Association with Breast Carcinoma}

UPSC rarely expresses oestrogen receptors (ER) and progesterone receptors (PR) \{Carcangiu, 1990\}. Whilst some studies have demonstrated association of development of UPSC with tamoxifen therapy \{Magriples, 1993\}, other studies were unable to reproduce these findings, observing a similar rate of type I and type II endometrial carcinoma in breast cancer patients regardless of tamoxifen use \{Barakat, 1994\}. Geisler et al \{Geisler, 2001\} found that patients with UPSC had an increased risk of either synchronous breast cancer or subsequently developing breast cancer. Since UPSC is not a hormonally driven tumour, this raises the question as to why these patients are at risk of developing breast cancer compared to other patients with endometrial cancer. In the study by Geisler et al \{Geisler, 2001\} there was no significant difference in the mean age of patients with UPSC versus EEC, therefore age alone could not account for the difference. A case report and a study of 12 women raised a possible link between carrying the BRCA1-2 mutations and the development of UPSC \{Lavie, 2000;Hornreich, 1999\}. Existing studies show conflicting results as to whether endometrial cancer in general and UPSC in particular have an increased incidence of BRCA 1-2 mutations \{Lavie, 2004; Goshen, 2000;Biron-Shental T, 2006\}. Although all of these studies have been small, with limited power to determine significance, the disparity in results suggests that the 
observed association between UPSC and breast cancer may be due to the presence of mutations in other cancer predisposing genes, such as P53 \{Goshen, 2000\}.

\subsubsection{Association with Lymphoma}

A case report by Chang et al \{Chang, 2004\} described a patient undergoing chemoradiotherapy for Stage IV non-Hodgkin's lymphoma who developed UPSC. They postulated that the depressed host immunity might have had a role in the development of UPSC. However, it is most likely that depression of the immune system plays a small role in the development of UPSC, as otherwise chronically immunosuppressed patients including renal transplant patients and those with HIV/AIDS also should have an increased incidence of UPSC and this has not been noted in the literature. In contrast, a recent paper demonstrated decreasing expression of HLA-DR and increasing expression of CD4 in normal endometrium, ECIS and UPSC respectively \{Tamiolakis, 2005\}. The authors suggested that low HLA-DR caused an "immune attract" mechanism. However, they also concluded that this probably played a minor role in the development of UPSC.

\subsection{Pathogenesis}

The exact pathogenesis of UPSC is unknown. However, recently many molecules including oncogenes and tumour suppressor genes have been demonstrated in association with UPSC. This section gives a brief overview of these molecules and proteins. 


\subsubsection{Oncogenes}

Oncogenes are derived from proto-oncogenes: cellular genes that promote normal growth and differentiation. They may be found on the cell membrane (as growth factor receptors), or in the cytoplasm involved in signal transduction or in the nucleus, where they play a role in the cell cycle.

\subsubsection{Growth Factor Receptors}

C-erbB-2 (also known as HER-2/neu) is a member of the epidermal growth factor receptor family (EGFRF). EGFR proteins function as cell surface receptors and comprise three separate regions; the extracellular ligand binding site, a hydrophobic transmembrane region and a highly conserved intracellular signalling area, which contains tyrosine kinase \{Prenzel, 2001\}. The tyrosine kinase constituent can be phosphorylated to initiate complex intracellular signalling cascades, which in turn control cell cycle, survival, proliferation and gene transcription \{Prenzel, 2001\}. The C-erbB-2 gene can be amplified causing increased responsiveness of the signalling pathways and thus increased cell division and proliferation of damaged cells. Alternatively deletions may occur in the gene resulting in permanent activation of the receptor and increased cell division and proliferation \{Prenzel, 2001; Moscatello, 1995\}. Studies have shown overexpression of C-erbB-2 and amplification of the gene in USPC \{Santin, 2002;Santin, 2003;Santin, 2005;Santin, 2005;Macwhinnie, $2004\}$. 


\subsubsection{Proteins Involved in Signal Transduction}

Platelet derived growth factor receptor (PDGFR) and Abl overexpression has been identified immunohistochemically in primary and recurrent EEC and UPSC \{Slomovitz, 2004\}. Both of these proteins play a part in signal transduction from the cell membrane receptor, through the cytoplasm to the nucleus. When PDGFR is activated, it activates ras, found on the inner surface of the cell membrane, which in turn is transformed into its activated state and thus triggers the MAP kinase pathway, and proliferation. Thus, in UPSC, when PDGFR is overexpressed, the ras pathway is increasingly activated. $\mathrm{Abl}$ is a member of the non-receptor associated tyrosine kinase family and has tyrosine kinase activity, which is regulated by inhibitory domains. The main drawback of immunohistochemical studies is that they can only determine the presence or absence of a protein. However, the study examining the immunohistochemical expression of PDGFR and Abl also demonstrated expression of phosphorylated PDGFR and Abl, suggesting that these proteins are present in UPSC in the activated state \{Slomovitz, 2004\}.

\subsubsection{Cell Cycle Regulators}

The ultimate outcome of all growth promoting stimuli is the entry of quiescent cells into the cell cycle. Therefore genes associated with the cell cycle and in particular with the G1/S interface are frequently altered in cancer. P27 belongs to the kinase inhibitor protein (KIP) family and this protein inhibits the cyclin dependant kinases (cdks) associated with the cyclins E and D \{Lloyd, 1999\}. These particular cyclin kinase complexes regulate cell proliferation by stimulating the phosphorylation of the retinoblastoma protein $(\mathrm{Rb})$, causing the release and activation of elongation 
factor 2 (E2F), a transcriptional factor. E2F facilitates the traverse of the G1/S checkpoint of the cell cycle. Figure 1.1 shows a diagram of the cell cycle courtesy of Cotran et al \{Cotran RS, 2006\} Copyright CC Elsevier 2005.

UPSC displays a high incidence of p27 alterations, suggesting that p27 abnormalities play an important role in the development of UPSC \{Schmitz, 2000\}. Cyclin D1 overexpression has also been noted in a small percentage of UPSC cases $\{$ Schmitz, 2000; Stamenkovic, 2000;Moreno-Bueno, 2004\}. Cyclin E overexpression also occurs in UPSC, and may be associated with gene amplification \{Cassia, 2003\}. High levels of cyclin E, cdk2 and cdk4 correlate with weak or absent ER expression and p16 and p21 expression is associated with low PR expression \{Milde-Langosch K, 2001\}.

Recent studies have demonstrated that loss of cables, a cdk binding protein, is associated with a high incidence of endometrial hyperplasia and endometrial adenocarcinoma. This has been demonstrated not only in low grade EEC, but also in high grade EEC, UPSC and clear cell carcinoma \{DeBernardo, 2005;Zukerberg, 2004\}. Cables plays a role in proliferation and differentiation of the cell and its activity appears to be increased by progesterone and decreased by oestrogen $\{$ Zukerberg, 2004\}.

\subsubsection{Tumour Suppressor Genes}

In normal cells tumour suppressor genes inhibit cell proliferation. Therefore loss of tumour suppressor genes causes an increase in cell proliferation. Tumour suppressor genes may be found on the cell surface, under the plasma membrane, in the cytoskeleton, cytoplasm and in the nucleus. 


\section{The Cell Cycle}

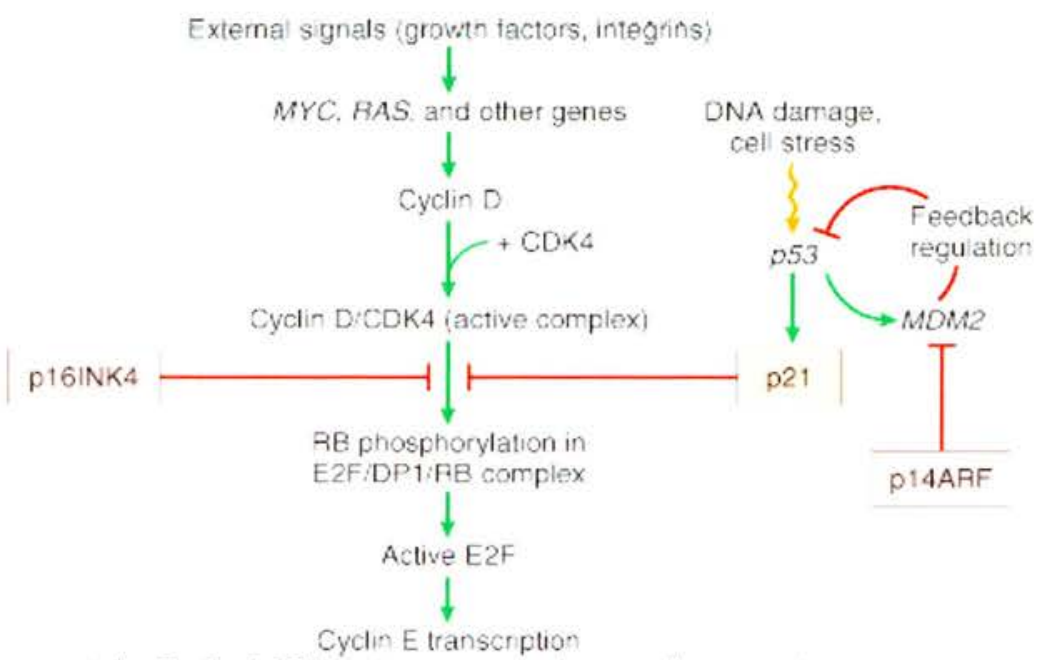

also Cyclin A, DNA poymerases, and many other genes:

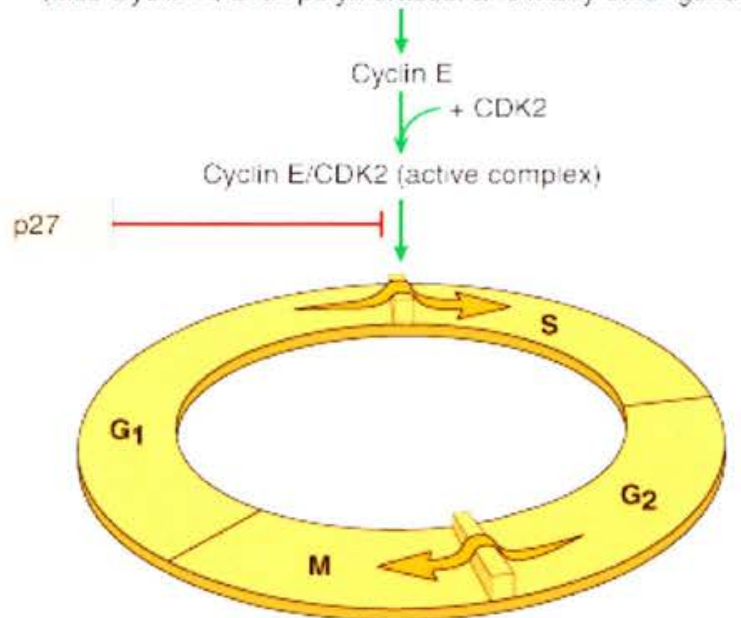

(c) Elsevier 2005 


\subsubsection{Cell Surface}

\subsection{Transforming Growth Factor $\beta$}

Transforming growth factor $\beta$ (TGF- $\beta$ ) is a member of the large family of structurally related cytokines that play an important role in controlling cell proliferation, differentiation, migration and apoptosis \{Derynck, 2001;Siegel, 2003\}. Somatic mutations in the TGF- $\beta$ signalling pathway are associated with loss of proliferative control, malignant progression, invasion and metastasis \{Vogelmann, 2005\}. TGF- $\beta$ binds to specific transmembrane serine/threonine kinase TGF- $\beta$ receptors such as type II (TGF- $\beta$ RII), which in turn transphosphorylates type I receptor (TGF- $\beta$ RI). Receptors convey signals across the plasma membrane through intracellular effectors such as SMAD proteins, which are translocated to the nucleus where they act as transcription factors \{Massague, 2000; Itoh, 2000;Miyazono, $2000\}$. SMAD proteins have been divided into 3 functional classes: receptor regulated (R-SMADS), common mediator (Co-SMAD), and inhibitory (I-SMADs) $\{$ Miyazono, 2000\}. Representatives of the first class are directly phosphorylated by TGF- $\beta$ RI and are involved in the various signalling pathways. SMAD2 and SMAD3 are effectors of TGF- $\beta$ /activin signalling while SMAD1, SMAD5 and SMAD8 are involved in bone morphogenetic protein (BMP) signalling. Co-SMAD is critical for TGF- $\beta$ /activin and BMP signalling cascades. Representatives of the third class of SMADs (SMAD6 and SMAD7) have the ability to inhibit the TGF- $\beta$ signalling pathway $\{$ Shi, 2001;Massague, 2000\}. TGF- $\beta$ can play multiple roles in human tumorigenesis, behaving as a tumour suppressor at early stages and a tumour promoter at late stages of carcinogenesis \{Reiss, 1999;Wong, 2001;Wakefield, 
2002\}. Studies showed TGF- $\beta$ RII protein level was higher and SMAD 2 and SMAD 4 mRNAs were lower in infiltrating endometrial carcinomas compared to Stage Ia EECs \{Piestrzeniewicz-Ulanska, 2004\}. Liu et al \{Liu FS, 2003\} showed that although $\mathrm{LOH}$ of chromosome 18q21 (the location of the SMAD4 gene) was frequent in endometrial carcinomas, immunohistochemistry showed that inactivation of the gene occurred infrequently. Both of these studies examined a mixed group of endometrial tumours and neither attempted to separate expression of type I and type II endometrial carcinomas. Therefore the role of TGF $\beta$ specifically in UPSC is still uncertain.

\subsection{E Cadherin}

E cadherin is the most common cadherin in epithelial cells. It is made from $4.5 \mathrm{~kb}$ mRNA as a $135 \mathrm{kDa}$ precursor polypeptide, which is processed rapidly by proteolytic cleavage to the mature $120 \mathrm{kDa}$ form $\{$ Shore, 1991 \}. The $\mathrm{E}$ cadherin gene $(\mathrm{CDH} 1)$ is found on chromosome 16q22.1 and it is thought to be a tumour suppressor gene, whose loss has been shown to cause tumour invasion and metastasis in various cancer models \{Christofori, 1999\}. In EEC loss of heterozygosity at 16q22 has been related to poor prognosis and $\mathrm{CDH1}$ mutations and promoter hypermethylation have been found in a small percentage of cases \{Kihana, 1996;Risinger, 1994;MorenoBueno, 2003;Saito, 2003\}. In addition to abnormalities in promoters of the E cadherin gene, or the gene itself, the E cadherin-catenin complex can be disturbed by the action of TGF- $\beta$. Vogelmann et al \{Vogelmann, 2005\} demonstrated that TGF- $\beta$ induced destabilisation of the $\mathrm{E}$ cadherin-catenin complex and thus dissociation of $\beta$ catenin from $\alpha$ catenin and the actin cytoskeleton. They also demonstrated that the 
mechanism involved phosphorylation of $\beta$ catenin by PTEN. Signalling through the cadherin-catenin complexes is involved in the regulation of EGFR distribution \{Deugnier, 1999\}. There is decreased expression of $\mathrm{E}$ cadherin in atypical hyperplasia compared to normal endometrium and even less in EEC \{Shih, 2004;Moreno-Bueno, 2003\}. Studies have also shown that loss of E cadherin expression increases with increasing grade of the endometrial carcinoma, such that the most poorly differentiated tumours have the least $\mathrm{E}$ cadherin expression \{Moreno-Bueno, 2003;Shih, 2004;Dvalishvili, 2005\}. Studies have shown conflicting results of $\mathrm{E}$ cadherin loss in type I, compared to type II endometrial carcinomas \{Demopoulos, 1999;Moreno-Bueno, 2003;Shih, 2004\}.

\subsubsection{Nucleus}

Numerous studies of P53 have been performed on endometrial carcinoma. P53 is involved in apoptosis, a complex energy dependent mechanism that eliminates unwanted cells selectively with minimal disturbance to surrounding cells $\{$ Kerr, $1972\}$. No p53 alteration is found in atypical hyperplasia, the precursor of EEC, whereas P53 alteration is common in both ECIS and UPSC \{Zheng, 1998 ;Busmanis, 2005 \}. These data suggest that p53 inactivation may occur at an early stage in the pathogenesis of UPSC. P21 is a downstream effector of p53 mediated G1 arrest after DNA damage and is sometimes used as an indicator of p53 function. Kovalev et al \{Kovalev, 1998\} demonstrated that although $78 \%$ of UPSC cases showed strong nuclear staining for $\mathrm{p} 53$, only $53 \%$ of these tumours had detectable p53 mutations. In addition, $70 \%$ of those tumours with mutated p53 had concomitant lack of p 21 expression consistent with transcriptionally inactive p53, whereas the other tumours 
had p21 staining suggesting p53 independent p21 expression. Of the cases which did not show any p53 mutation, more than half still showed strong positive nuclear staining, but lacked concomitant p21 expression. These findings suggest that nuclear expression of p53 in UPSC can be associated with both mutation dependent and independent type of p53 inactivation. In addition, p53 expression is associated with loss of ER and PR expression \{Moll, 1996\}. A recent paper demonstrated expression of p63 in ECIS and UPSC. P63 is a p53-homologous gene which codes for multiple protein isotypes, including full length forms with p53 like actions and truncated forms with p53 antagonistic actions \{Idrees MT, 2006\}. The authors also demonstrated that p63 expression correlated with p53 and therefore postulated that p63 mutations also occurred early in the development of UPSC.

\subsubsection{Genes that Regulate Apoptosis}

In addition to $\mathrm{p} 53$, genes and gene-protein products such as $\mathrm{Bcl}-2, \mathrm{Bax}$ and $\mathrm{Bcl}-\mathrm{x}$ are involved in the control of apoptosis $\{$ Crescenzi, 2000;Lu, 1996\}. Bcl-2 is a protooncogene, first identified from the t (14-18) translocation, which occurs in follicular lymphoma $\{$ Chen-Levy, 1989$\}$. Bax is a $21-\mathrm{kDa}$ protein that shares homology with Bcl-2 and forms homodimers \{Oltvai, 1993\}. It is thought that Bcl-2 suppresses apoptosis through heterodimerisation with $\mathrm{Bax}$, but that $\mathrm{Bax} / \mathrm{Bax}$ homodimerisation triggers programmed cell death $\{$ Yin, 1994\}. Through an alternate splicing mechanism the $\mathrm{Bcl}-\mathrm{x}$ gene encodes two proteins that have opposing functions \{Boise, 1993\}. Bcl- $\mathrm{x}_{\mathrm{L}}$ is the long form and inhibits apoptosis. The short form, Bcl$\mathrm{x}_{\mathrm{S}}$, triggers apoptosis. Studies have shown that expression of $\mathrm{Bcl}-2$, Bax and $\mathrm{Bcl}-\mathrm{x}_{\mathrm{L}}$ varies between normal endometrium, atypical hyperplasia, EEC, ECIS and UPSC 
\{Bozdogan, 2002;Busmanis, 2005\}. Bcl-2 expression in normal endometrium was significantly less than ECIS or UPSC, but no difference was found between ECIS and UPSC, suggesting that Bcl-2 loss occurs early in the pathogenesis of UPSC \{Busmanis, 2005\}.

\subsubsection{Genes that Regulate DNA Repair}

UPSC less commonly shows microsatellite instability (MSI) compared to EEC \{King, 1995\}. Hereditary Nonpolyposis Colorectal Carcinoma (HNPCC) is an inherited condition, characterised by germ line mutations in the mismatch repair genes MLH1 and MSH2. Abnormal methylation or mutations in these genes lead to MSI. Broaddus et al \{Broaddus, 2006\} showed that in those patients with HNPCC who developed EEC, hypermethylation of MLH1 had occurred leading to MSI. However, in those patients with HNPCC, who went on to develop non-EEC, Mutations of MSH2 were more likely to be found.

\subsubsection{Karyotypic Changes.}

Loss of heterozygosity $(\mathrm{LOH})$ at chromosome $1 \mathrm{p}$ occurs in more than $63 \%$ UPSC, rarely in EEC and not in ovarian serous papillary carcinoma (OSPC). This suggests the presence of a tumour suppressor gene on chromosome 1p, important in UPSC tumorigenesis \{Arlt, 1996;Herzog, 2001\}.

\subsection{Presenting Features}

Most patients with UPSC present in a similar way to those with EEC. The most common presenting symptom is abnormal vaginal bleeding. Abdominal symptoms 
such as bloating, pain or change in weight are occasionally seen and these symptoms are more common in patients presenting at a later stage \{Podratz, 2003\}.

\subsection{Macroscopic appearances}

UPSC has similar macroscopic features to all endometrial carcinomas. It usually forms exophytic lesions within the endometrial cavity, with varying degrees of myometrial invasion. Occasionally UPSC may arise in a polyp or form a polyp. Hui et al \{Hui, 2005\} studied 40 cases of UPSC, where macroscopically, the tumour was confined to the endometrium. They found that UPSC involved an endometrial polyp in 35 cases $(88 \%)$ and in 18 cases $(45 \%)$ extra-uterine tumour was present. In those cases, which were surgically staged as Stage Ia, either confined to a polyp or the endometrium, the overall survival after an average of 26 months follow up was $94 \%$. They did not find any difference in outcome within the Stage Ia category where tumour was either confined to a polyp or was confined to the endometrium. There are no macroscopic features present, which can help differentiate between USPC and any other type of endometrial carcinoma.

\subsection{Microscopic Appearances}

UPSC was first recognised as a clinical variant in 1947 but it was not until 1982 that Hendrickson et al published a full clinical and morphological description of the lesion \{Hendrickson, 1982\}. Their criteria for diagnosis included complex papillary architecture with tufted stratification of the lining epithelium, marked nuclear polymorphism, high nuclear to cytoplasmic ratio, macronuclei and high mitotic rate. Solid areas could also be identified \{Hendrickson, 1982\}. Psammoma bodies can be 
present in up to $33 \%$ of UPSC (See Figures 2.1 to 2.4 in chapter 2). UPSC is often found along with other subtypes of endometrial carcinoma including endometrioid and clear cell variants. Sherman et al \{Sherman, 1992\} suggested that the term UPSC should be used when it comprised at least $25 \%$ of the tumour. However, to date no study has been performed determining the exact amount of UPSC needed to be present to give the poor prognosis associated with "pure" UPSC. Some tumours are diffusely infiltrative, whereas others have a pushing border. The significance of this is uncertain.

\subsubsection{Immunohistochemistry}

Like all endometrial carcinomas, immunohistochemistry (IHC) shows UPSC to express CK7 and focal Ca125. UPSC is associated with overexpression of p53 and IHC for $\mathrm{p} 53$ is sometimes helpful in confirming the diagnosis. However, antibodies to $\mathrm{p} 53$ are not helpful in distinguishing between EEC and UPSC as both carcinomas express p53 to a variable degree, with increasing expression seen in any type of higher grade tumours \{Macwhinnie, 2004;King, 1995\}. GLUT1, a membrane bound facilitative glucose transporter, whose expression is associated with tumour hypoxia, has been demonstrated in ECIS and UPSC \{Idrees MT, 2006\}. Therefore it may have a role in distinguishing ECIS from normal or reactive endometrial epithelium. However, as it has also been demonstrated in atypical endometrial hyperplasia and EEC, it would not be useful in distinguishing UPSC from EEC. Expression of WT1, a tumour suppressor gene, can be used to distinguish OSPC from UPSC as OSPC uniformly expresses nuclear WT1 and UPSC shows either no expression or cytoplasmic expression of WT1 \{Euscher, 2005\}. 


\subsection{Spread}

\subsubsection{Pattern of Spread}

UPSC resembles OSPC in its microscopic appearance and pattern of spread, but has a different molecular pathology \{Hendrickson, 1982;King, 1995;Herzog, 2001 ;Zorn KK, 2005\}. UPSC has a propensity for lymphovascular space involvement, stromal invasion and peritoneal spread $\{\mathrm{Lim}, 2001\}$. It is upstaged at the time of surgery in $60 \%$ of cases \{Gehrig, 2001;Jeffrey, 1986;Kato, 1995;Goff, 1994;Dunton, 1991\}.

\subsubsection{Mechanism of Spread}

Invasion and metastasis involves many complex interactions and processes. Figure

1.2 shows the metastatic cascade, courtesy of Cotran et al \{Cotran RS, 2006\} Copyright (C) Elsevier 2005. 


\section{The Metastatic Cascade}

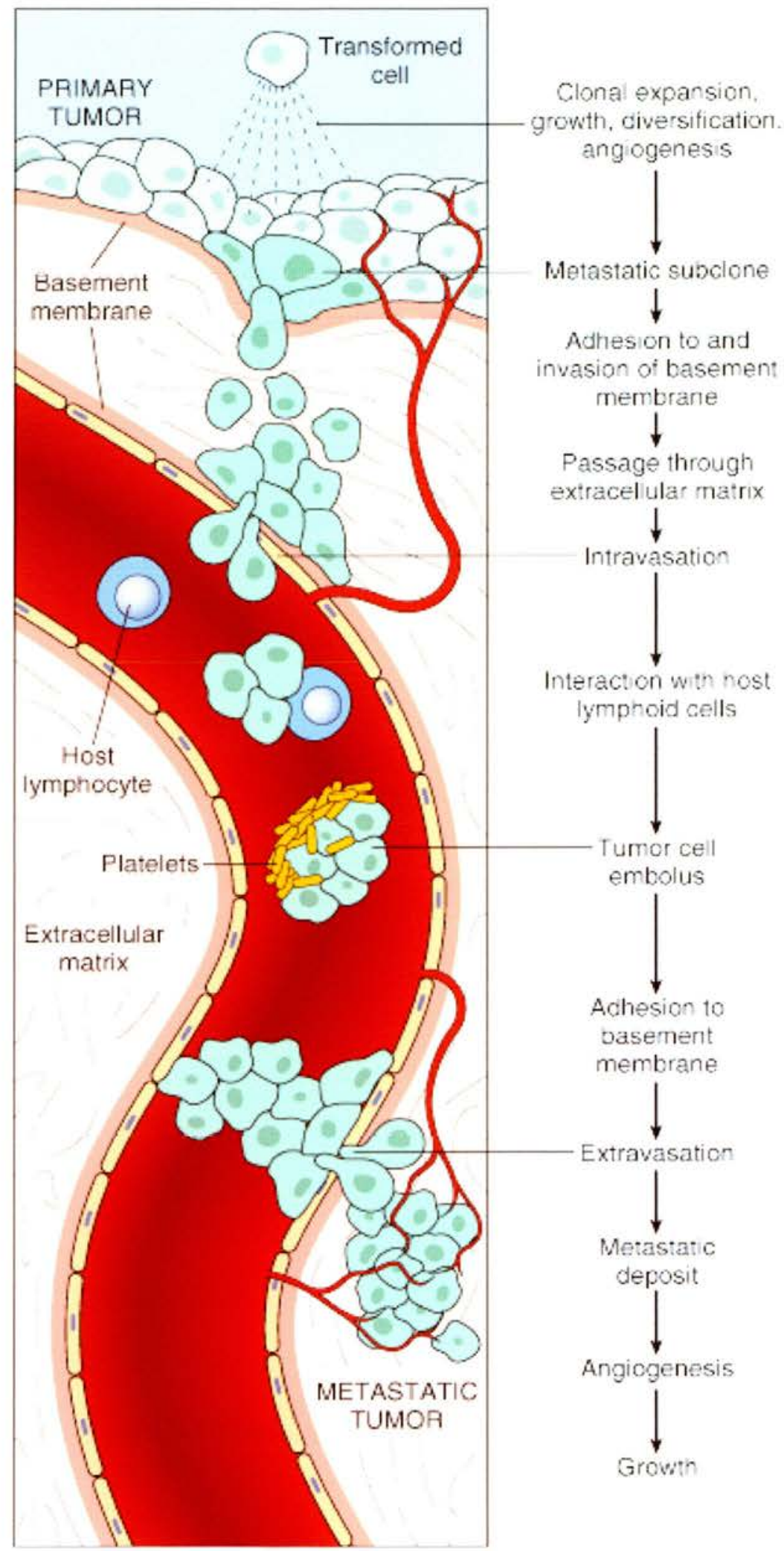

(C) Elsevier 2005 


\subsubsection{Detachment from Adjacent Cells}

The role of E cadherin as a tumour suppressor gene has been previously discussed. This section focuses on its possible role in invasion of tumour cells. Cadherins are a widely distributed family of cell-cell adhesion molecules with varying degrees of tissue specific expression. E cadherin is found in all normal epithelia. As transmembrane cell adhesion molecules involved in cell-cell interactions, cadherin binding is homotypic and calcium dependent \{Nose, 1988;Matsuzaki, 1990\}. Intracellularly $\mathrm{E}$ cadherin is linked to members of the catenin family ( $\beta, \gamma$, or $\alpha$ catenin) through which it is connected to the actin cytoskeleton \{Nathke, 1994;Hinck, 1994;Jou, 1995\}. Cadherin-catenin complexes have roles in intercellular communication and modulation of cell function in both normal and malignant tissues. Failure to assemble the E cadherin-catenin complex or properly connect to the actin cytoskeleton results in loss of cell adhesion.

Decreased E cadherin expression levels have been observed in some "non EECs" \{Moreno-Bueno, 2003\}, and also has been associated with an increased risk of development of distant metastases \{Pijnenborg, 2004\}. However, nuclear localization of $\beta$ catenin (associated with mutations in the $\beta$ catenin gene) and mutations in the adenomatous polyposis coli (APC) gene were not predictive for recurrent disease. The exact role of $\mathrm{E}$ cadherin has not been fully elucidated and more work is needed in this area.

\subsubsection{Attachment to Matrix Components}

To penetrate the surrounding extracellular matrix (ECM), the tumour cells must first adhere to the ECM components. There is considerable evidence that receptor 
mediated attachment of tumour cells to laminin and fibronectin is important for invasion and metastasis \{Price JT, 1997;Ziober BL, 1996\}. Normal epithelial cells express high affinity receptors for basement membrane (BM) laminin that are polarised to their basal surface. In contrast some carcinoma cells have many more receptors and they are distributed all round the cell membrane and there appears to be correlation between the density of receptors and invasion in colon and breast carcinoma $\{$ Ziober BL, 1996\}. In addition to laminin receptors tumour cells also express integrins that can serve as receptors for many components of the ECM including fibronectin, laminin collagen and vitronectin \{Vizirianakis, 2001;Yao, 1996\}. Studies examining in vivo integrin expression in normal endometrium showed that $\beta 1$ integrin expression was mostly seen in endometrial stromal cells \{Castelbaum, 1997\}. In addition, in vitro studies showed that progesterone treatment of oestradiol-primed cells resulted in increased expression of the $\alpha 1 \beta 1$ collagenlaminin receptor and suppression of the $\alpha v \beta 3$ vitronectin receptor \{Castelbaum, $1997\}$. Lessey et al \{Lessey, 1995\} found alteration of integrin expression between benign and malignant endometrial epithelium, with the $\alpha 5 \beta 1$ integrin, most commonly seen on benign endometrial stromal cells, being found in almost $20 \%$ of cases of endometrial carcinoma. They also showed that integrin expression correlated with steroid receptor status, as well as with grade, stage and depth of invasion \{Lessey, 1995;Ziober BL, 1996\}.

\subsubsection{Invasion of Extracellular Matrix}

Degradation of the ECM is an essential element of angiogenesis, cellular invasion and tumour metastasis. Matrix metalloproteinases (MMP), a family of zinc- 
dependent endoproteinases, are widely accepted to play a role in these processes \{Chang, 2001;Vihinen, 2002\}. MMPs are normally expressed at a very low level in adult tissues, except in tissues that undergo remodelling such as cycling endometrium and wound healing. However, loss of control of MMP expression has been implicated in a number of diseases such as rheumatoid arthritis, osteoarthritis, chronic wounds and cancer \{Chang, 2001;Vihinen, 2002;Stamenkovic, 2000;Parks, 1999;John, 2001;Jiang, 2002\}. Based on their structure and substrate specificity MMPs are divided into several groups that include collagenases (MMP-1, MMP-3 and MMP-8), gelatinases (MMP-2 and MMP-9), stromeolysins (MMP-3, MMP-10, MMP-11), matrilysins (MMP-7, MMP-26), metalloelastase (MMP-12) and membrane type matrix metalloproteinases (MT-MMPs) \{Chang, 2001

;Vihinen, 2002;Stamenkovic, 2000;Parks, 1999;John, 2001;Jiang, 2002 \}.

The expression of MMPs is highly regulated at transcription, translation and secretion of latent enzyme, proenzyme activation and inactivation levels. The catalytic activity of MMPs is specifically controlled at least in part by their physiological inhibitors, the tissue inhibitors of MMPs (TIMPs), composed of TIMP1, $-2,-3$ and -4 \{Chang, 2001;Kugler, 1999;Jiang, 2002\}. TIMP-1, -2 and -4 are secreted in soluble forms whereas TIMP-3 is insoluble due to binding to ECM proteins \{Chang, 2001;Vihinen, 2002;Stamenkovic, 2000;Parks, 1999;John, 2001;Kugler, 1999;Jiang, 2002\}. Although TIMP -4 appears to have restricted tissue expression and function, TIMP expression is found in many tissues and is regulated in coordination with MMPs. In addition to regulating MMPs, TIMPs have been demonstrated to have angiogenic and growth factor-like activity \{Parks, 1999;John, 2001;Kugler, 1999;Jiang, 2002;Stetler-Stevenson, 1999\}. 
In the female reproductive tract tissues the expression of several MMPs and TIMPs is associated with menstruation, abnormal uterine bleeding, ovulation, embryo implantation, cervical ripening and parturition \{Martelli, 1993;Rodgers, 1994;Matrisian, 1992\}. Altered expression of MMPs (MMP-1, -2, -3, -7, -8, -9, -13) and TIMPs (TIMP-1, -2 and -3) have been shown to occur in endometrial adenocarcinoma and the results indicate increased expression of MMP-9 and MMP-7 is associated with myometrial invasion and lymph node metastasis respectively \{Yabushita, 2000;Ueno, 1999;Moser, 1999\}. In contrast MMP-2 expression is used to help differentiate high grade from low-grade endometrial carcinoma and MMP-9 in differentiating normal from sarcomatous endometrial stroma \{Iurlaro, 1999; Liokumovich, 1999; Inoue, 1997\}. Park et al \{Park, 2001\} demonstrated that cells from an endometrial adenocarcinoma cell line invaded by recruiting MMP-2 secreted by endometrial stromal cells and this was enhanced by the presence of beta oestradiol.

MMP-26 and TIMP-4 expression is elevated in endometrial carcinomas with the highest expression correlating with deeper myometrial invasion and grade 3 (high grade) tumours. There is no difference in expression between grade 3 EEC and USPC \{Tunuguntla, 2003\}.

\subsection{Treatment}

The most effective treatment for UPSC is surgery, and then depending on the stage of the disease, adjuvant chemotherapy, radiotherapy or both may be given. However, due to the relative rarity of the disease no chemotherapeutic regimes have been fully ratified in patients with UPSC. 


\subsubsection{Surgery}

In 1988 the International Federation of Gynaecology and Obstetrics (FIGO) changed the staging criteria for endometrial carcinoma from a clinical to surgical system. To appropriately stage endometrial carcinoma by the FIGO system, the surgeon must perform a total extrafascial hysterectomy and bilateral salpingo-oophorectomy, obtain peritoneal washings, and sample any suspicious pelvic or para-aortic lymph nodes. Several authors have shown that $40 \%$ to $60 \%$ of clinical Stage I UPSC are upstaged at the time of surgical staging and lesions clinically confined to the endometrium are upstaged in approximately $60 \%$ of cases \{Gehrig, 2001; Jeffrey, 1986;Kato, 1995;Goff, 1994\}. Several studies have shown the presence of lymph node metastases in up to $50 \%$ of cases at the time of surgery \{Huh, 2003;Slomovitz, 2003;Goff, 1994\}. Therefore the use of prognostic factors appropriate for EEC including depth of myometrial invasion and lymph vascular space invasion may not apply to UPSC \{Carcangiu, 1997;Carcangiu, 1992;Goff, 1994;Cirisano, 1999; Geisler, 1999; Gallion, 1989;Lee, 1991\}.

The 1988 FIGO system does not include omental evaluation as part of the surgical staging criteria for endometrial carcinoma. However, this is frequently performed in cases where UPSC has been diagnosed preoperatively as UPSC and OSPC have a similar pattern of intra abdominal spread with a propensity to spread to the upper abdomen. Gehrig et al \{Gehrig, 2003\} performed a study on 52 women with UPSC who had had omentectomy as part of their surgical staging. Thirty-four of the omenta were macroscopically and microscopically benign, 2 contained microscopic metastases and 16 were macroscopically and microscopically involved with tumour. 
The sensitivity of a visually negative omentum was $89 \%$. The authors concluded that, with a microscopic metastasis rate of $4 \%$, surgical sampling does not need to be included in the routine surgical staging of UPSC. However this would mean that 1 in 25 patients with UPSC would be under staged. Full surgical staging does appear to decrease local recurrences but using surgery as the sole therapy in UPSC is not useful due to the high recurrence rates, which mostly occur in the upper abdomen \{Hendrickson, 1982;Jeffrey, 1986;Goff, 1994;Sutton, 1987\}.

\subsubsection{Radiation Therapy}

The current rationale of treatment of endometrial carcinoma is to consider adjuvant pelvic radiotherapy in those patients with high-risk disease endometrial carcinoma (positive retroperitoneal lymph nodes, high histological grade or deep myometrial invasion). This is thought to reduce the risk of local recurrence, but has demonstrated less effect on overall survival as these patients usually die of distant disease \{Gehrig, 2004;Elit, 2002;Bristow, 1999\}. Huh et al \{Huh, 2003\} showed a similar overall survival in those patients with Stage I and II UPSC who were managed conservatively and those who received adjuvant radiotherapy suggesting that adjuvant radiotherapy may not have a role in those patients where the tumour is confined to the uterus. Whole abdominal pelvic irradiation (WART) has not been extensively studied, and consensus has not been reached regarding the benefits of this approach, but it is recommended, following resection due to the risk of upper abdominal dissemination $\{$ Lim, 2001\}. However studies examining the survival of patients treated with post operative WART have shown conflicting results \{Mallipeddi, 1993;Gibbons, 1991;Frank, 1991;Mehta, 2003\}. 


\subsubsection{Hormonal Therapy}

Progesterone therapy may be used empirically to treat metastatic EEC, as this is often a PR and ER positive tumour. However this has not been extensively used for patients with UPSC, as UPSC is usually an ER, and PR negative tumour \{Umpierre, 1994; Carcangiu, 1990\}.

\subsubsection{Systemic Chemotherapy}

Despite the microscopic similarity of UPSC to OSPC, UPSC does not show the same degree of chemosensitivity. However, the similarity of pattern of spread and recurrence to OSPC has led researchers evaluate similar treatments \{Bristow, 1999\}.

\subsubsection{Platinum Based Chemotherapy}

The most extensively evaluated treatment option in patients with UPSC is platinumbased chemotherapy, most commonly using cisplatin. Platinum-based agents act by cross-linking DNA, making it impossible for proliferating cells to replicate their chromosomes for mitosis. The cross-linked DNA activates DNA repair mechanisms, which ultimately induce apoptosis. Response rates associated with cisplatin-based chemotherapy range from $10 \%$ to $33 \%$ \{Price, 1993;Chambers, 1996;Levenback, 1992\}. However the combination of cisplatin, doxorubicin and cyclophosphamide chemotherapy is less effective in UPSC than in ovarian carcinoma \{Price, 1993; Chambers, 1996\}. Doxorubicin is a cytotoxic antibiotic and cyclophosphamide acts by alkylating DNA, thus damaging it and interfering with cell replication. To date, the results of intra-peritoneal cisplatin have failed to show dramatic results \{Chambers, 1996\}. 


\subsubsection{Taxol}

Paclitaxel (Taxol), a member of the taxane group of drugs, is another treatment of ovarian cancer, which has also had some success in the treatment of UPSC. Single agent paclitaxel showed a tumour response in $77 \%$ of patients \{Ramondetta, 2001 \}, and used in a neo-adjuvant fashion with cisplatin produced objective responses in 8 out of 9 patients \{Zanotti, 1999\}.

\subsubsection{Topotecan}

Topotecan is an established treatment in recurrent ovarian cancer, with response rates of $13 \%$ to $33 \%$ \{McGuire, 2000; Swisher, 1997;Bookman, 1998\}. Topotecan acts by blocking the action of topoisomerase I, an enzyme critical for cellular replication and repair. A pilot study assessing a 5-day regimen of topotecan as first line therapy in 15 women with UPSC found that 12 patients received topotecan as first-line therapy and 3 patients received it as second line treatment after failure of platinum therapy \{Gore, 2002\}. At median follow up of 13 months, 11 of the 12 patients who received first line topotecan $(93 \%)$ remained in remission. Of the 3 patients receiving second line topotecan, 2 died and the other patient remained disease free at the end of the trial.

\subsubsection{Herceptin}

Several studies have showed amplification of the HER-2/neu (c-erbB2) oncogene in UPSC \{Macwhinnie, 2004;Santin, 2003;Santin, 2002;Santin, 2005\}. In addition, Santin et al \{Santin, 2005\} demonstrated that those UPSC patients with c-erbB2 amplification had a poorer prognosis than those without c-erbB2 amplification. On the basis of these findings as well as those of previous reports showing a positive in vivo correlation between the efficacy of Herceptin ${ }^{R}$ therapy and the level of HER2/neu overexpression by tumour cells, Herceptin ${ }^{R}$ therapy may be a novel strategy in 
a significant number of patients with chemotherapy resistant recurrent or metastatic UPSC \{Santin, 2002\}.

\subsubsection{Imatinib Mesylate}

Imatinib mesylate is a tyrosine kinase inhibitor that specifically targets c-kit, Abl and PDGFR. It has been shown to be effective in the treatment of patients with chronic myeloid leukaemia and gastrointestinal stromal tumours. These cancers are characterized by activating mutations of $\mathrm{Abl}$ and c-kit tyrosine kinases respectively. As both Abl and PDGFR are expressed in primary and recurrent UPSC, imatinib mesylate may be a useful treatment for these patients \{Slomovitz, 2004\}.

\subsubsection{Summary}

The benefit to patients from such multimodality therapy remains uncertain because no significant improvement after adjuvant therapy has been noted in the last decade \{Bancher-Todesca, 1998; Tay, 1999\}. However, there is clearly a need to continue investigating possible treatments due to the dismal prognosis of UPSC.

\subsection{Prognosis}

The outcome of endometrial carcinoma is influenced by the initial stage, and grade of the tumour. While early stage endometrial carcinoma is associated with relatively good cure rates, advanced stage disease is characterized by a poorer prognosis. Overall survival for women with surgically staged endometrial carcinoma ranges from $70 \%$ to $90 \%$ for those with Stages I and II disease and $5 \%$ to $60 \%$ for those with III and IV disease. However, when grouped by histological type, overall survival for women with EEC is $76 \%$ while survival for women with UPSC is much worse, ranging from 0 to $45 \%$ \{Zanotti, 1999;Price, 1993; Gitsch, 1995;Creasman, 
2003; Chambers, 1987;Carcangiu, 1992;Abeler, 1990\}. Approximately $75 \%$ of women with EEC present with disease clinically confined to the uterus (Stage I), whereas $37-66 \%$ of women with UPSC tend to present with more advanced stage disease \{Creasman, 2003; Gitsch, 1995\}. Most series report survival rates of 35\%$50 \%$ for Stages I and II and $0-15 \%$ for Stages III and IV UPSC \{Jeffrey, 1986;Dunton, 1991;Gallion, 1989;Sutton, 1987;Abeler, 1990;Piura, 1998;Bristow, 2001\}. Despite the best treatment attempts, relapse rates are reported to be as high as $50 \%$ to $80 \%$ \{Christman, 1987; Chambers, 1987;Ramondetta, 2001;Hendrickson, $1982\}$.

Many studies have been performed in the attempt to identify factors that might be useful in predicting outcome of these patients. Population based data from the American National Cancer institute found that although the incidence of endometrial cancer was higher in white patients, black patients actually had a worse outcome \{Hicks, 1998\}. Santin et al \{Santin, 2005\}, found that earlier deaths were seen in those UPSC patients that overexpressed c-erbB2, black patients and those over 65 years of age at presentation. However, multivariate Cox regression showed that although short survival was significantly associated with c-erbB2 expression it was not associated with either race or age, suggesting that the c-erbB2 amplification caused much of the race disparity in survival in the patient population.

High serum levels of interleukin-6 (IL-6) are associated with, and expressed by, UPSC \{Bellone, 2005;Santin, 2005\}. IL-6 is a pleiotropic cytokine which is involved in the immune system, acute phase responses and has various effects on haematopoiesis \{Hirano, 1998\}. IL-6 regulates cell growth of a variety of human cancers and inhibits the antitumour effects of the immune system. High serum levels 
of IL-6 correlate with shorter survival in patients with renal cell, prostatic and ovarian carcinoma \{Blay, 1992;Nakashima, 2000;Scambia, 1995;Bachelot, 2003\}.

The kallikrein family is large and its members are all proteases, which encode for trypsin-like or chymotrypsin-like serine proteases. Kallikrein -6 and -10 have been detected both in vivo and in vitro in UPSC \{Santin, 2006;Santin, 2005\}. Kallikrein 10 is implicated in the growth and invasion of breast, ovarian and prostate cancer \{Diamandis, 2002; Goyal J, 1998\}. Kallikrein-6, -10 and IL-6 may be useful in monitoring early disease recurrence and response to therapy.

Busmanis et al \{Busmanis, 2005\} did not find that p53 was a useful predictor for either survival time or disease stage. However, there is evidence that $\mathrm{p} 73$ expression profiles and p53 status are useful in differentiating between patients with good and poor prognosis. Despite P73 having significant homology to p53, it is not thought to be a tumour suppressor gene. The TP73 gene has $5 \mathrm{~N}$-terminal isoforms including TAp73, DeltaNp73 and DeltaN'p73. Delta Np73 derives from an alternative promoter in intron 3 and lacks the transactivation domain of full length Tap73. It counteracts transactivation function, apoptosis and growth suppression mediated by wild-type p53 and TAp73 and confers drug resistance to wild type p53 harbouring tumour cells. Conversely down regulation of endogenous DeltaNp73 levels inhibits its suppressive action and enhances p53- and Tap73-mediated apoptosis \{Zaika AI, 2002\}. Using a support vector machine algorithm Becker et al \{Becker K, 2006\} demonstrated that TAp73, DeltaNp73 and DeltaN'p73 were significantly upregulated in all gynaecological tumours. 


\subsection{Summary}

Uterine papillary serous carcinoma is a highly aggressive variant of endometrial carcinoma and accounts for approximately $10 \%$ of cases, but a much smaller proportion of Stage I endometrial carcinomas. It has a grim prognosis. This chapter has described what is currently known about UPSC and has focussed on the aetiology, pathogenesis and treatment of this aggressive cancer. Recently there have been several reviews of series of UPSC patients, predominantly focussing on the molecular pathology of UPSC or current treatment strategies, and all suggesting the value of a larger scale clinical trial \{Slomovitz, 2003;Ramondetta, 2001;Zanotti, 1999;McGuire, 2000;Swisher, 1997\}. To date there is no clinically proven treatment strategy for UPSC, and patients with this tumour receive ad hoc therapy, which has no proof of efficacy. A large scale clinical trial is important because all previous studies have been limited by the relative rarity of the disease; therefore the small numbers of patients seen in all centres and also the small numbers involved in these reviews have limited the power of the studies. 


\section{Chapter 2}

\section{Materials and Methods for Chapters 5, 6 and 7.}

\subsection{Tissues}

\subsubsection{Identification of Cases}

Suitable cases were identified in three ways. Firstly, a search of the APEX computer system was made from 1992 to 2004 . The codes used to identify suitable cases were T-84000 (endometrium), T-82920 (Total hysterectomy), T-82000 (Uterus), T-82900 (Uterus and cervix) and M-80103 (carcinoma), M-83803 (endometrioid carcinoma) and M-82603 (papillary carcinoma). The data that was recovered also included the patient's name, date of birth and the numbers accorded to the specimens by the Department of Pathology. Secondly a separate search was performed on the Department of Oncology Gynaecology database from 1994 to 2003, where the search parameter was any patients in whom a diagnosis of uterine papillary serous carcinoma (UPSC) had been made. This data was predominantly used for the audit into the diagnosis and management of UPSC. Lastly the paper records of the weekly regional Gynae-oncology multidisciplinary meeting were examined and those cases that on review were described as either UPSC or Grade 3 endometrioid endometrial carcinoma (EEC) were selected. The results from all three searches were combined. For the purposes of the audit on the diagnosis and management of UPSC, all of the cases from 1994 to 2003, selected by the oncology database were used. For the creation of the tissue microarrays (TMAs), all cases where blocks and remaining tissue were available were used. No patients or cases were excluded for any other reason. 


\subsubsection{Patient Demographics}

\subsubsection{Uterine Papillary Serous Carcinoma}

The demographic details for those patients selected for the audit on the diagnosis and management of UPSC are described in Chapter 4. Seventy-eight patients were identified for the TMAs. Their demographics are described here. The median age of the patients was 68 years (range 49-89). Nine patients (12\%) had a past medical history of breast cancer. No patients developed breast cancer after the time of diagnosis of UPSC. The FIGO staging system \{Creasman, 1990\} was used in describing the stage of disease. Table 2.1 shows the FIGO staging system. The numbers and percentages of each patient with each stage of disease are presented in Table 2.2. Hui et al \{Hui, 2005\} showed that UPSC confined to either an endometrial polyp or the endometrium had good prognosis. However, as only 4 of our UPSC cases were Stage Ia, these were not treated as a separate group.

\subsubsection{Endometrioid Endometrial Carcinoma}

Twenty patients with FIGO grade 3 EEC were identified. The median age of the patients was 66 years (range 46-85 years). No patients had either a past history of breast carcinoma, or developed it subsequently. The numbers and percentages of each patient with each stage of disease are presented in Table 2.2. 
Table 2.1 FIGO Staging for Carcinoma of the Endometrium

\begin{tabular}{|c|c|}
\hline Stage I & Tumour confined to uterine corpus \\
\hline $\begin{array}{l}\text { IA } \\
\text { IB } \\
\text { IC }\end{array}$ & $\begin{array}{l}\text { Limited to endometrium } \\
\text { Invasion less than half of myometrium } \\
\text { Invasion more than half of } \\
\text { myometrium }\end{array}$ \\
\hline Stage II & Tumour involves cervix \\
\hline IIB & $\begin{array}{l}\text { Endocervical glandular (mucosal) } \\
\text { involvement only } \\
\text { Invasion into cervical stroma }\end{array}$ \\
\hline Stage III & \\
\hline $\begin{array}{l}\text { IIIB } \\
\text { IIIC }\end{array}$ & $\begin{array}{l}\text { Tumour invades serosal of corpus uteri } \\
\text { and/or adnexae and/or positive } \\
\text { cytological findings } \\
\text { Vaginal metastases } \\
\text { Metastases to pelvic and/or paraaortic } \\
\text { lymph nodes }\end{array}$ \\
\hline Stage IV & \\
\hline IV A & $\begin{array}{l}\text { Tumour invades bladder mucosa } \\
\text { and/or bowel mucosa } \\
\text { Distant metastases, including intra- } \\
\text { abdominal metastasis and/or inguinal } \\
\text { lymph nodes }\end{array}$ \\
\hline
\end{tabular}


Table 2.2 Demographic Details of Patients Used to Create Tissue Microarrays.

\begin{tabular}{|c|c|c|}
\hline & UPSC & EEC \\
\hline $\begin{array}{l}\text { Median Age } \\
\text { (Range) }\end{array}$ & $\begin{array}{l}68 \text { years } \\
(49-89) \text { years }\end{array}$ & $\begin{array}{l}66 \text { years } \\
(45-85) \text { years }\end{array}$ \\
\hline $\begin{array}{l}\text { History of Breast } \\
\text { Carcinoma } \\
\text { (Patients) }\end{array}$ & 9 & 0 \\
\hline Stage I & $29(37 \%)$ & $14(70 \%)$ \\
\hline IA & 4 & 0 \\
\hline IB & 17 & 9 \\
\hline IC & 8 & 5 \\
\hline Stage II & $21(27 \%)$ & $1(5 \%)$ \\
\hline IIA & 10 & 0 \\
\hline IIB & 11 & 1 \\
\hline Stage III & $17(22 \%)$ & $4(20 \%)$ \\
\hline IIIA & 12 & 4 \\
\hline IIIB & 2 & 0 \\
\hline IIIC & 3 & 0 \\
\hline Stage IV & $11(14 \%)$ & $1(5 \%)$ \\
\hline Total Patients & 78 & 20 \\
\hline
\end{tabular}




\subsubsection{Tissue Samples}

Archival tissue samples were selected from the Royal Infirmary of Edinburgh Department of Pathology, the slides were reviewed by HM and ARWW (a subspecialist gynaecological pathologist), the diagnosis of UPSC or EEC was confirmed and the appropriate paraffin blocks were selected. All tissue had been routinely fixed in $4 \%$ buffered formaldehyde and processed into paraffin blocks. The diagnostic features used to classify cases as UPSC and EEC are well described and are as follows $\{$ Robboy $\}$ :

\subsubsection{Uterine Papillary Serous Carcinoma}

At low power UPSC shows either papillary architecture or solid areas where the papillae are composed of broad fibrovascular cores with secondary or tertiary papillary processes and with prominent dropping off of cells into the lumen. The cells are generally rounded and the nuclei are pleomorphic with occasional multinucleation. In addition the nuclei tend to be centrally placed rather than lying adjacent to the basement membrane. Mitoses and foci of necrosis are common. Psammoma bodies are found in some cases (Figures 2.1 to 2.4). 
Figure 2.1

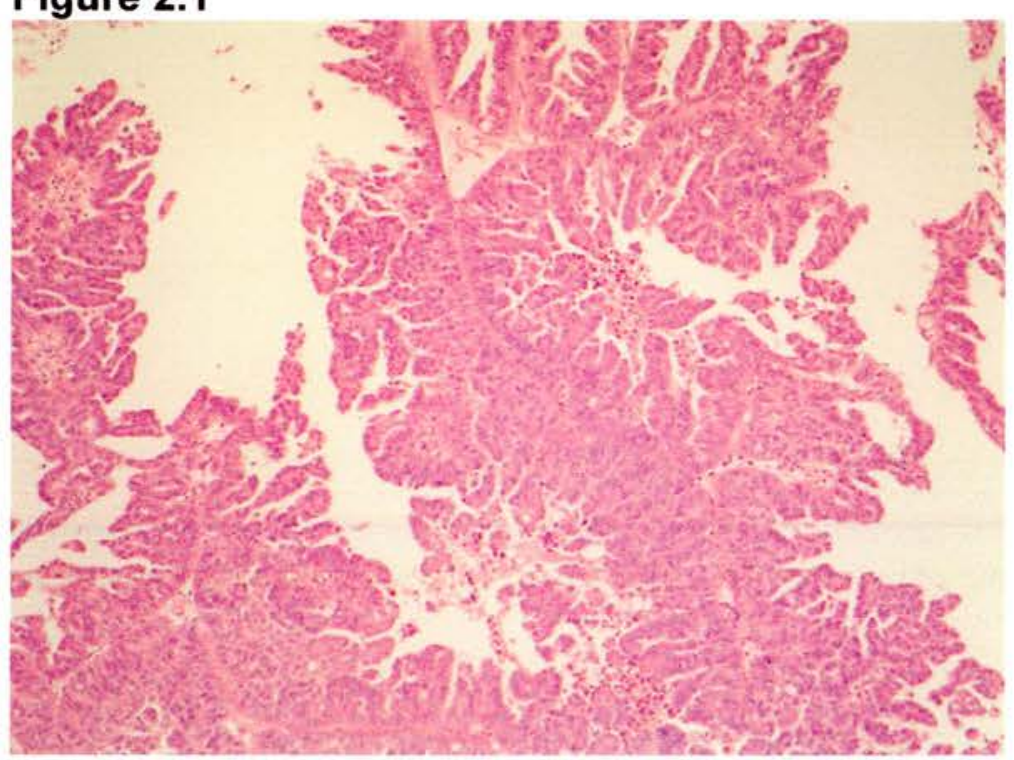

Figure 2.2



Figure 2.1. Papillary Architecture of UPSC (H\&E x10).

Figure 2.2. Solid Architecture of UPSC (H\&E x10). 
Figure 2.3

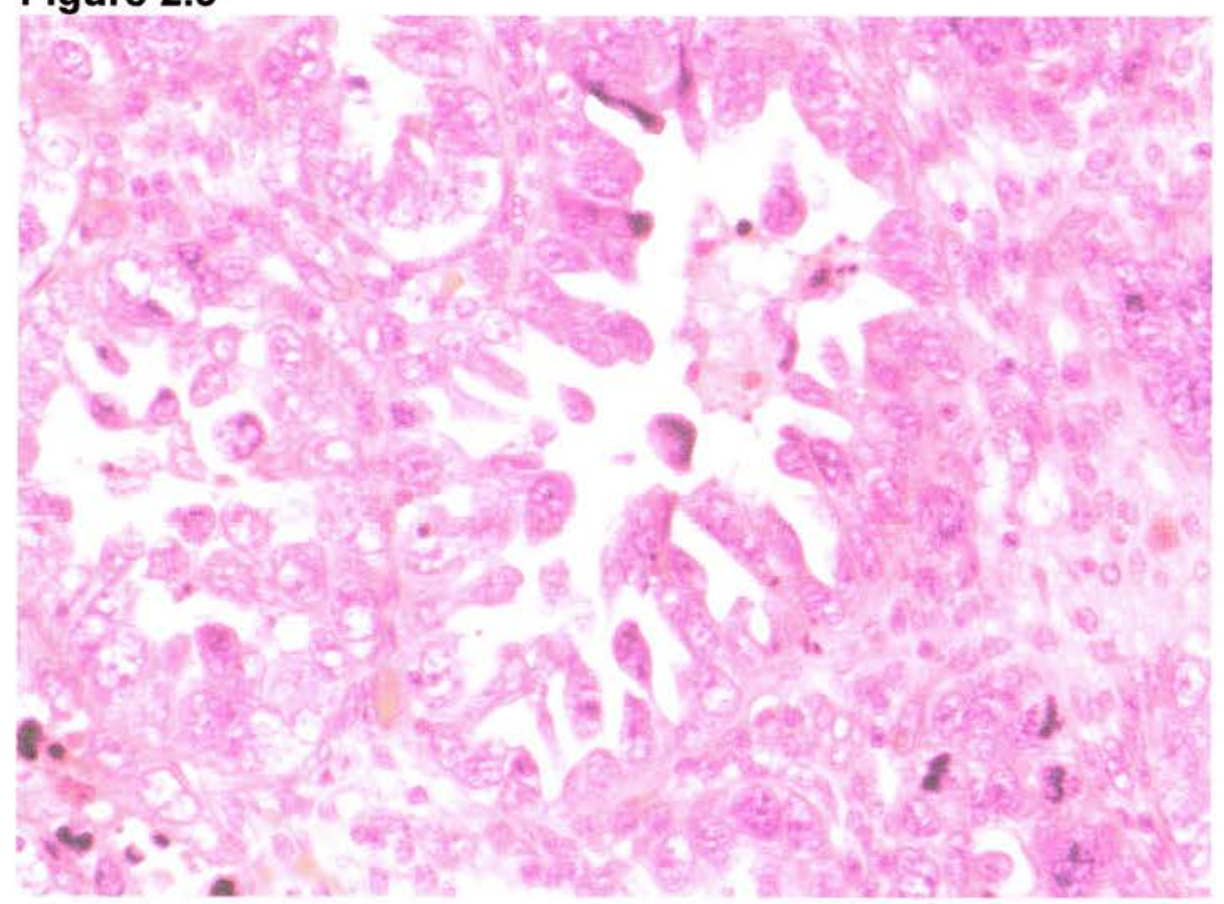

Figure 2.4

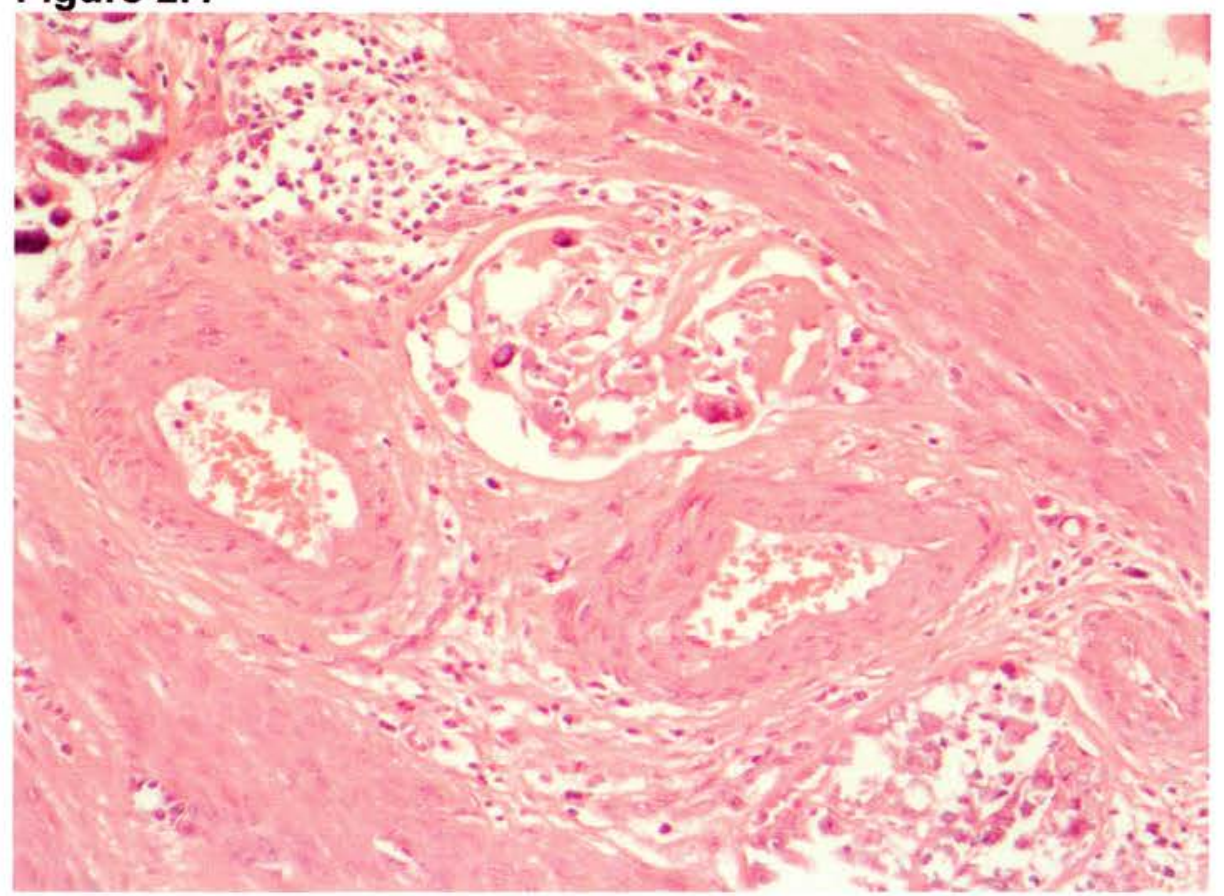

Figure 2.3. Pleomorphic Nuclei forming a papillary pattern in UPSC (H\&Ex40).

Figure 2.4 Psammoma Bodies Lying within Tumour Adjacent to Myometrial Arteries (H\&Ex20). 


\subsubsection{Endometrioid Endometrial Carcinoma}

EEC can range from low to high grade. The well-differentiated (Grade 1) carcinomas can be difficult to distinguish from atypical complex hyperplasia. EEC typically has at least a focus of glandular differentiation lined by stratified columnar cells, with oval nuclei arranged at right angles to the basement membrane. As the carcinoma becomes more poorly differentiated (Grade 3), the prominent gland formation decreases and solid areas and sheets of cells are more apparent \{Tavassoli, 2006\} (Figures 2.5 to 2.8 ). EEC is typically divided into three grades, namely; Grade 1, Grade 2 and Grade 3, which are also referred to as well-, moderately- and poorlydifferentiated respectively. The classification of grading developed by the World Health Organisation \{Scully\} is used and Table 2.3 shows the grading of EEC with architecture being the most important determinant. Significant nuclear atypia, described as round nuclei, variation in shape and size, variation in staining, hyperchromasia, coarsely clumped chromatin, prominent nucleoli, frequent mitoses and abnormal mitoses, will increase the grade by one, irrespective of the architectural features. Only cases of Grade 3 (high grade) EEC were selected for comparison with UPSC for this study.

At least one area from the viable central part and invasive edge of the tumour were then marked by a circle (Figure 2.9). 
Figure 2.5

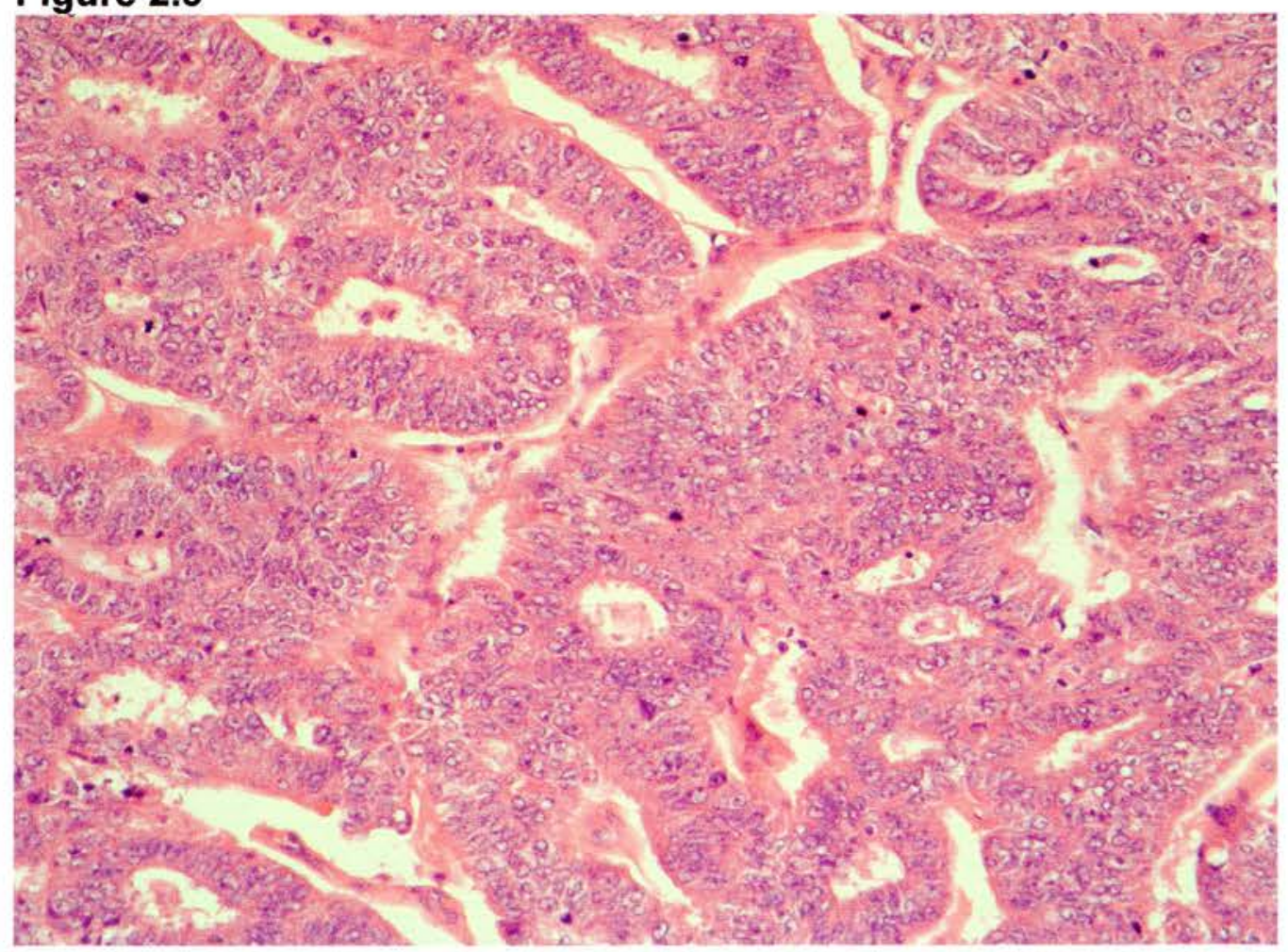

\section{Figure 2.6}

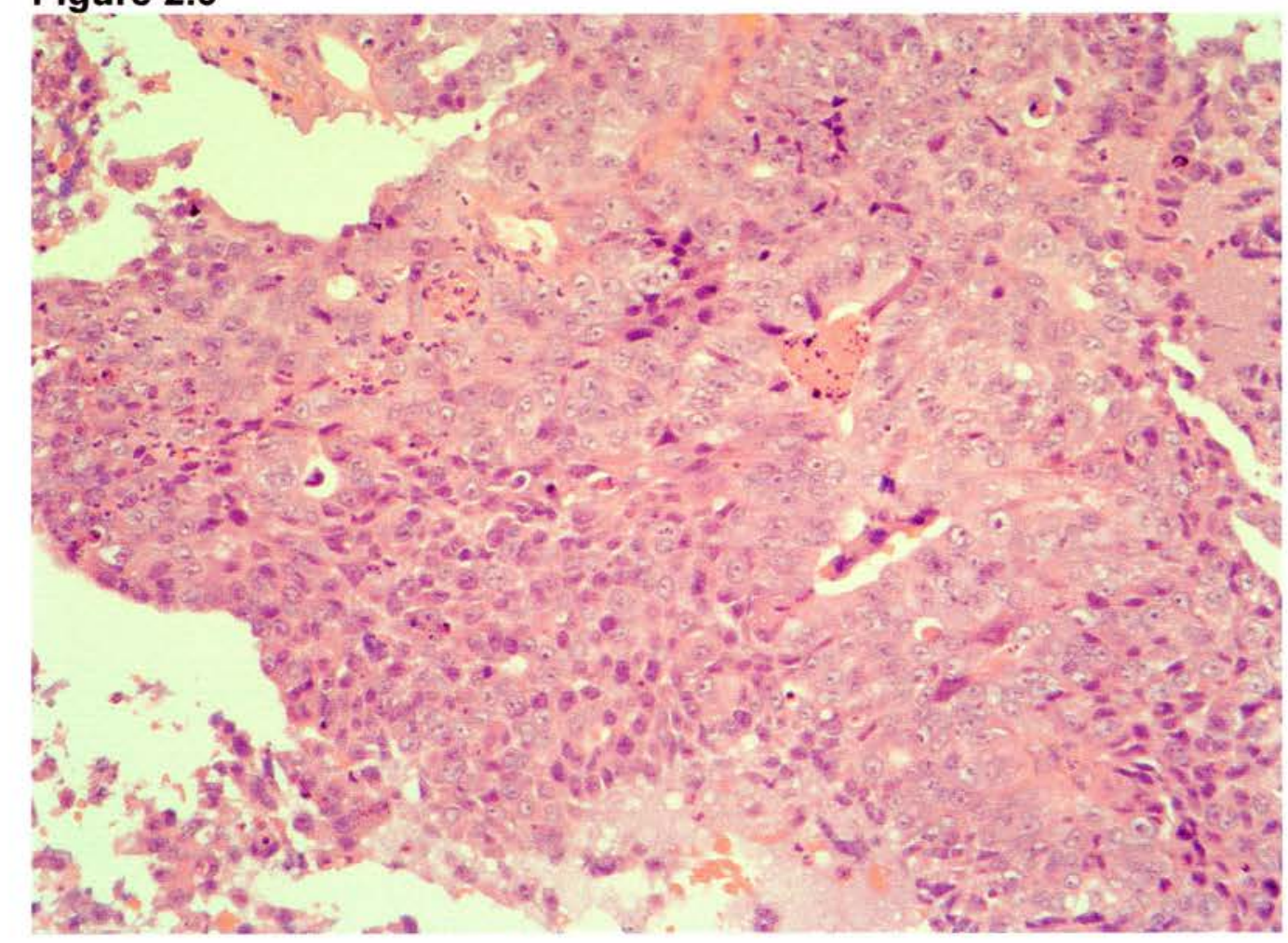

Figure 2.5. Classical Gland Formation of EEC (H\&E x20).

Figure 2.6. Solid Architecture of EEC (H\&E x10). 


\section{Figure 2.7}

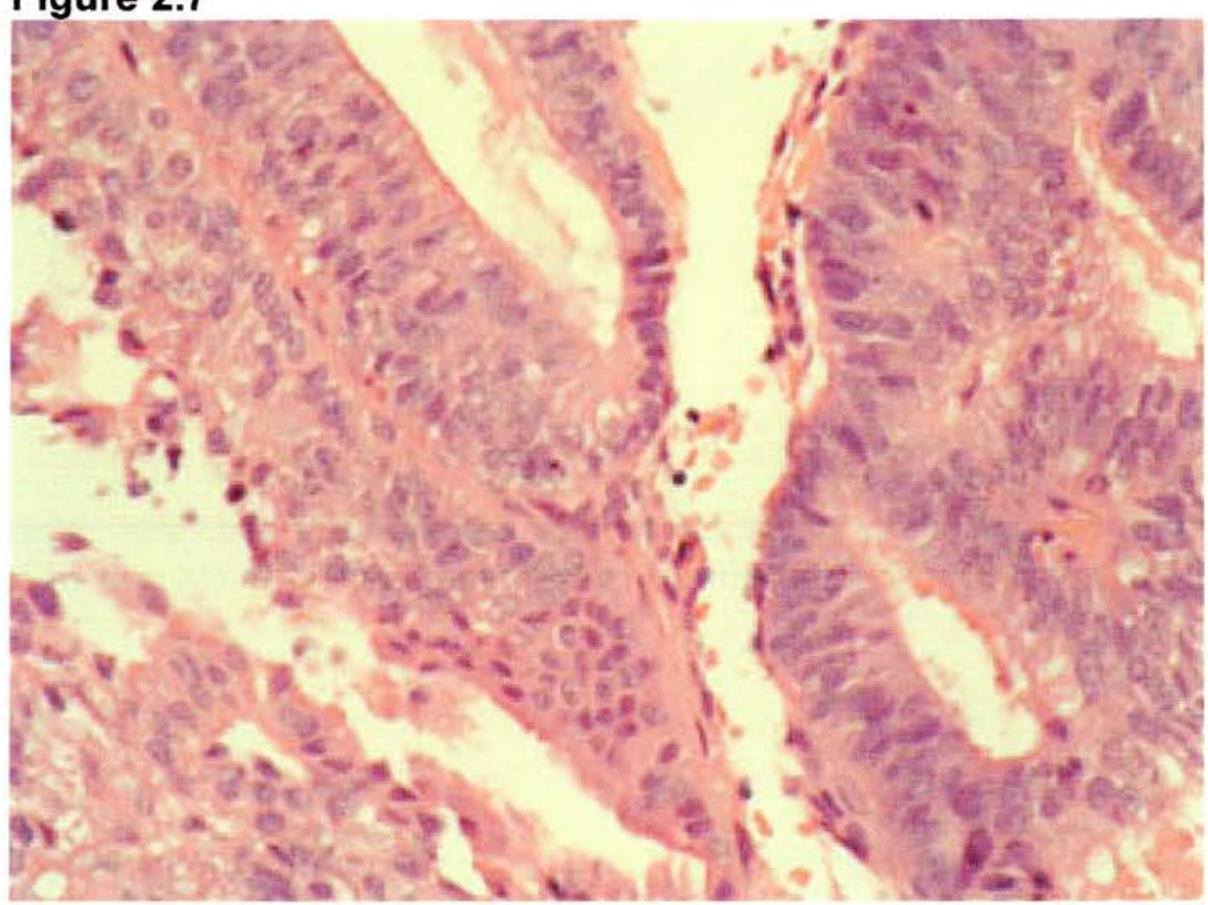

\section{Figure 2.8}

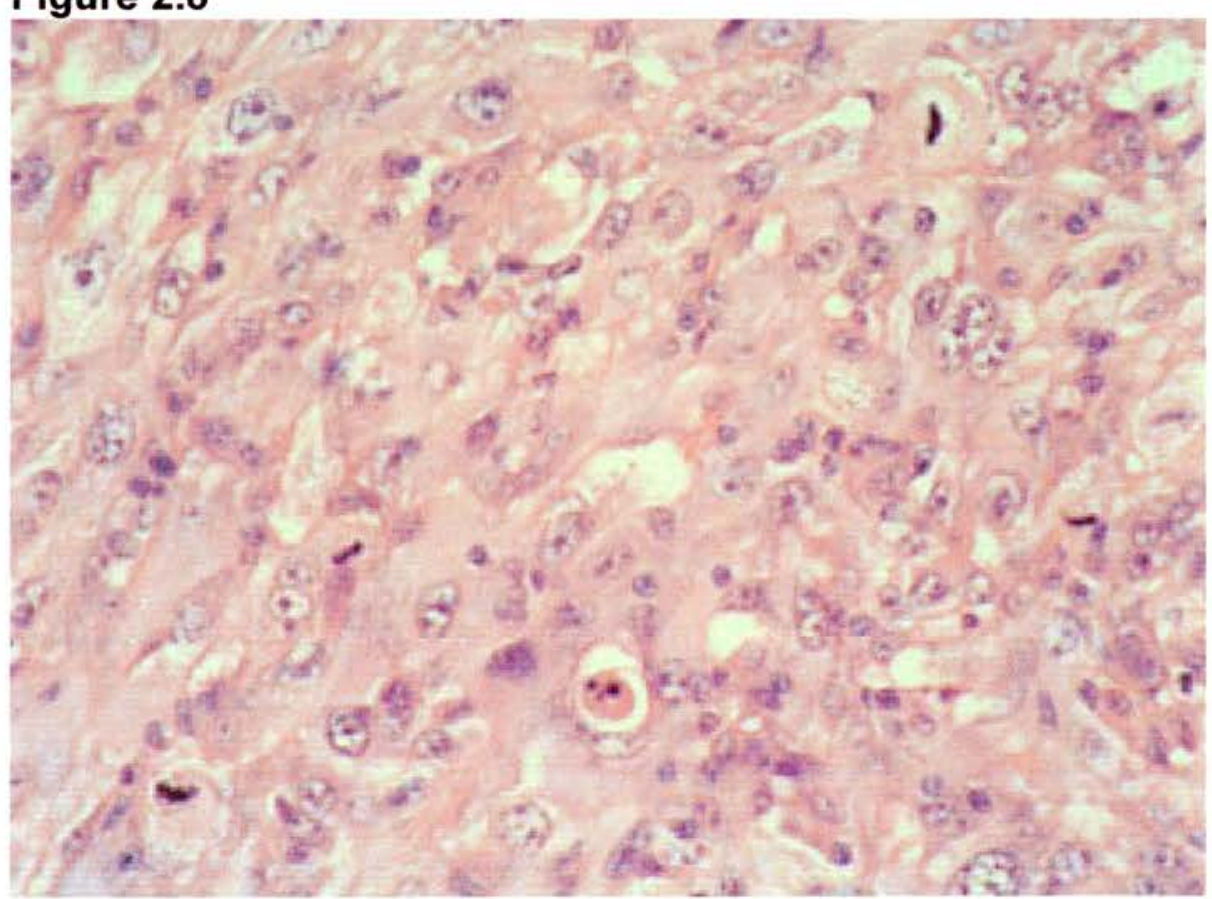

Figure 2.7. Stratification of cells in glands in Grade 1 EEC (H\&E x20).

Figure 2.8. Pleomorphic nuclei in Grade 3 EEC (H\&E x10). 
Figure 2.9

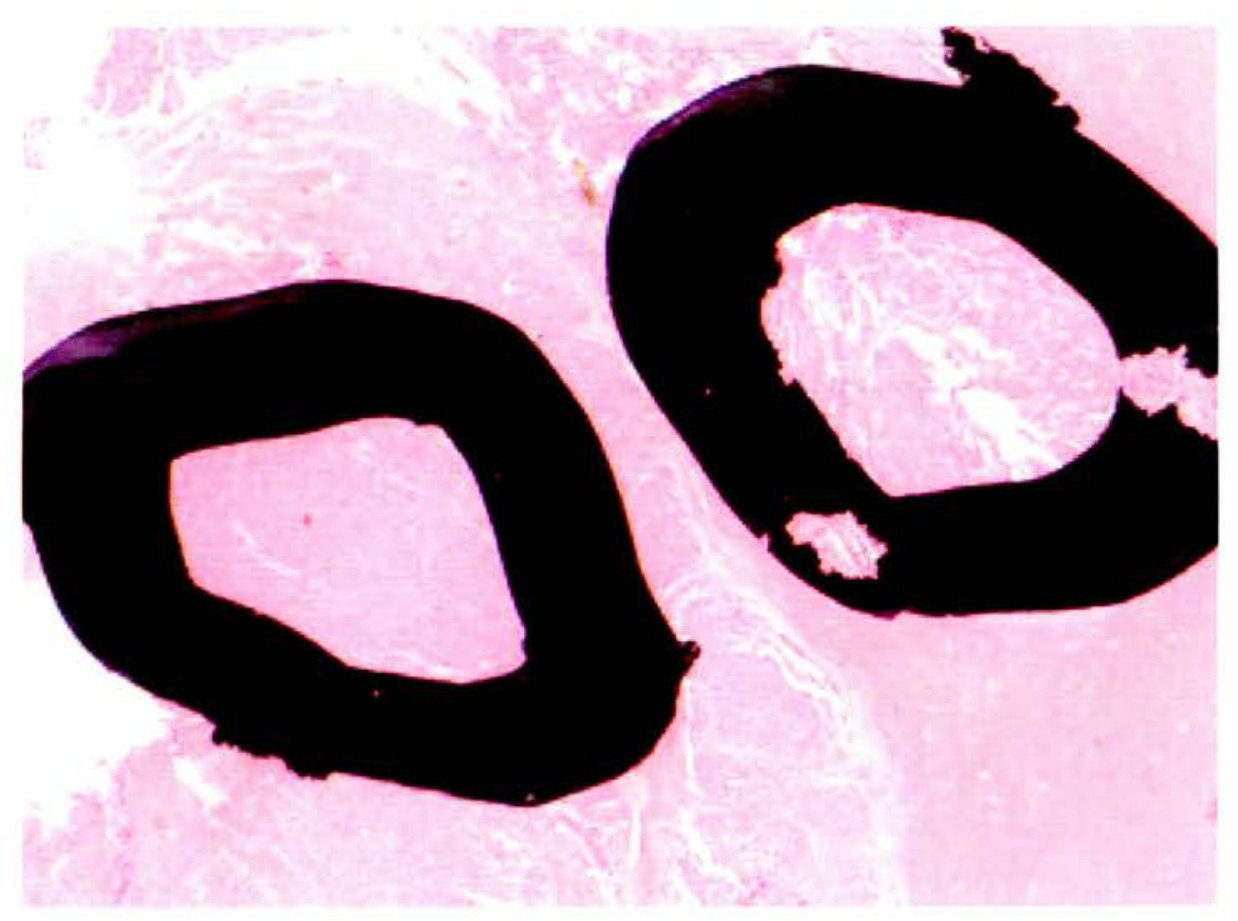

Figure 2.9. Circles showing area to be sampled by Beecher Instrument. At least three cores can be taken from within each circle (Magnificationx4). 
Table 2.3 Grading of Endometrial Carcinoma.

\begin{tabular}{|l|l|l|l|}
\hline & Grade 1 & Grade 2 & Grade 3 \\
& (Well & (Moderately & (Poorly \\
& Differentiated) & Differentiated) & Differentiated) \\
\hline Percentage of & $>95 \%$ & $>50 \%$ & $<50 \%$ \\
Glands & & & \\
\hline Percentage of & $<5 \%$ & $<50 \%$ & $>50 \%$ \\
Solid Growth & & & \\
\hline Nuclear atypia & \multicolumn{2}{|l}{ Increase grade by 1. } \\
\hline
\end{tabular}




\subsection{Tissue Microarray}

A tissue microarray (TMA) instrument by Beecher Instruments was selected (Figure 2.10). In order to construct the microarray, empty paraffin blocks of a depth $5-10 \mathrm{~mm}$ were produced. Cores of wax, $0.8 \mathrm{~mm}$ diameter were extracted from the empty blocks and replaced with cores of $0.6 \mathrm{~mm}$ diameter taken from the tissue blocks at sites corresponding to the previously selected areas on the H\&E slides (Figure 2.11). Two cores were taken from each area marked on the slide, so that at least two cores were taken from each case. The cores were punched at $1 \mathrm{~mm}$ intervals, at least 2 cores per case, and the numbers of cores taken from each case ranged from 2 to 10 . A grid system with each core having a coordinate reference ( $\mathrm{X}$ axis, $\mathrm{Y}$ axis) was used to allow cross-reference between core location and parent case.

Once the TMAs were complete the blocks were sealed in a $60^{\circ} \mathrm{C}$ oven for 10 minutes. 


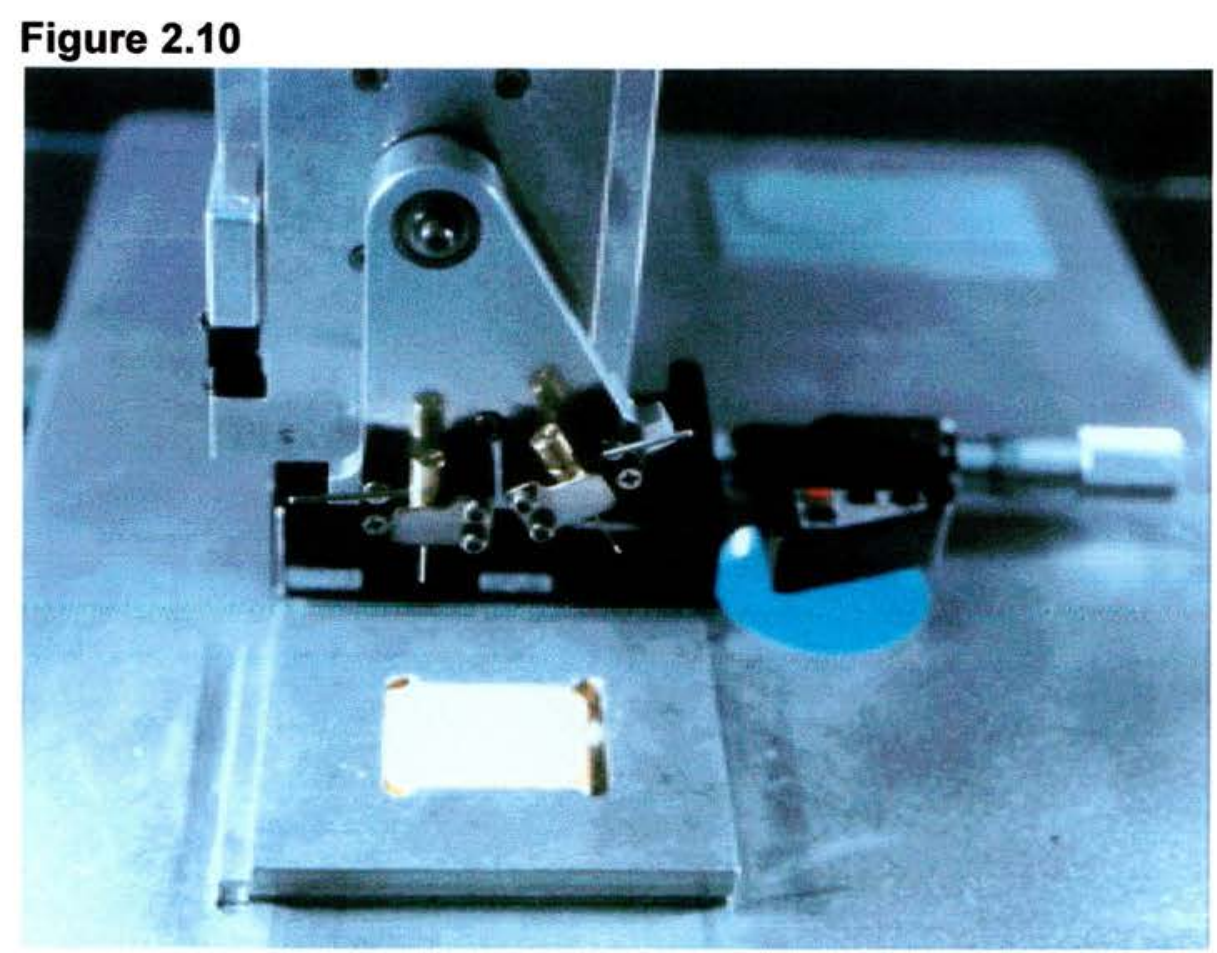

Figure 2.11

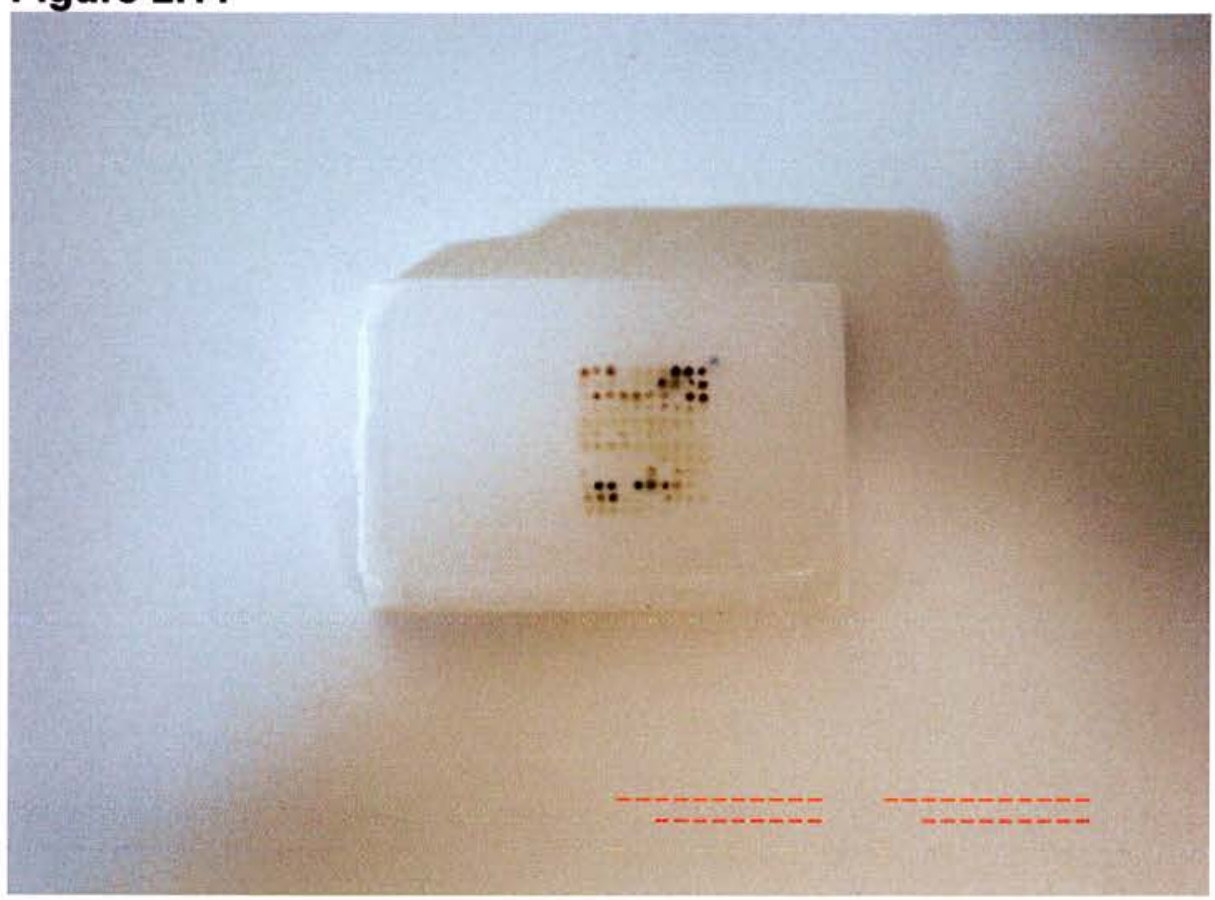

Figure 2.10. Beecher instrument used to create Tissue Microarrays courtesy of Skacel et al.

Figure 2.11. Tissue Microarray Block used for EEC cases. 


\subsection{Immunohistochemistry}

From the TMA blocks, sections were cut at $3 \mu \mathrm{m}$ thickness and mounted on positively charged capillary action slides (Dako, Copenhagen, Denmark) and incubated at $60{ }^{\circ} \mathrm{C}$ overnight. The slides were dewaxed and rehydrated prior to antigen retrieval. Table 2.4 shows a summary of the details of the antibodies used.

\subsubsection{P53}

Antigen retrieval was performed by immersing the test slides and a case of breast carcinoma (as the positive control) in $0.01 \mathrm{M}$ ethylenediamine tetraacetic acid (EDTA), PH8.0 and microwaving at high power for 15 minutes. The primary antibody was the D-07 clone (Dako, Denmark) and was used at dilution 1:50. Negative controls were obtained by omitting the primary antibody. All slides were stained on the Dako TechMate ${ }^{\mathrm{TM}} 500$ plus, using the Dako ChemMate ${ }^{\mathrm{TM}}$ EnVision $^{\mathrm{TM}}$ Detection kit and the standard operating procedure for routine immunohistochemistry.

\subsubsection{ER}

Antigen retrieval was performed by immersing the test slides along with a slide of breast carcinoma (as the positive control) in 0.01M EDTA, PH8.0 and microwaving at high power for 15 minutes. The primary monoclonal antibody was the $6 \mathrm{~F} 11 / 2$ clone (Novocastra, UK) and was used at dilution 1:200. Negative controls were obtained by omitting the primary antibody. All slides were stained on the on the Dako TechMate ${ }^{\mathrm{TM}} 500$ plus, using the menapath supersensitive detection kit polymer HRP and the standard operating procedure for routine immunohistochemistry. 
Table 2.4. Primary Antibodies and Antigen Retrieval Conditions used in

IHC.

\begin{tabular}{|c|c|c|c|c|c|}
\hline Antigen & Manufacturer & Clone & Dilution & $\begin{array}{l}\text { Antigen } \\
\text { Retrieval }\end{array}$ & Control \\
\hline P53 & Dako, Denmark & D-07 & $1: 50$ & $\begin{array}{l}\text { EDTA } \\
\text { Microwave }\end{array}$ & Breast Carcinoma \\
\hline ER & $\begin{array}{l}\text { Novocastra } \\
\text { Laboratories, UK }\end{array}$ & $6 \mathrm{~F} 11 / 2$ & $1: 200$ & $\begin{array}{l}\text { EDTA } \\
\text { Microwave }\end{array}$ & Breast Carcinoma \\
\hline MMP-2 & $\begin{array}{l}\text { Novocastra } \\
\text { Laboratories, UK }\end{array}$ & 17B11 & $1: 40$ & $\begin{array}{l}\text { EDTA } \\
\text { Microwave }\end{array}$ & Appendix \\
\hline MMP-7 & $\begin{array}{l}\text { Chemicon } \\
\text { International, } \\
\text { UK }\end{array}$ & 1D1 & $1: 25$ & None & Breast Carcinoma \\
\hline MMP-9 & $\begin{array}{l}\text { Novocastra } \\
\text { Laboratories, UK }\end{array}$ & $15 \mathrm{~W} 2$ & $1: 80$ & $\begin{array}{l}\text { EDTA } \\
\text { Pressure } \\
\text { Cooker }\end{array}$ & Kidney \\
\hline PR & Dako, Denmark & PgR 636 & $1: 100$ & $\begin{array}{l}\text { EDTA } \\
\text { Microwave }\end{array}$ & Breast Carcinoma \\
\hline Galectin 3 & $\begin{array}{l}\text { Novocastra } \\
\text { Laboratories, UK }\end{array}$ & $9 \mathrm{C} 4$ & $1: 150$ & $\begin{array}{l}\text { EDTA } \\
\text { Microwave }\end{array}$ & $\begin{array}{l}\text { Papillary Carcinoma } \\
\text { of Thyroid }\end{array}$ \\
\hline CD98 & Santa Cruz & $\begin{array}{l}\text { Polyclonal } \\
\text { IgG }\end{array}$ & $1: 200$ & $\begin{array}{l}\text { Pressure } \\
\text { cooker } \\
\text { Microwave }\end{array}$ & Tonsil \\
\hline E Cadherin & Dako, Denmark & $\mathrm{NCH}-38$ & $1: 25$ & $\begin{array}{l}\text { EDTA } \\
\text { Pressure } \\
\text { Cooker }\end{array}$ & Tonsil \\
\hline P Cadherin & $\begin{array}{l}\text { Novocastra } \\
\text { Laboratories, UK }\end{array}$ & $56 \mathrm{Cl}$ & $1: 100$ & $\begin{array}{l}\text { EDTA } \\
\text { Microwave }\end{array}$ & Placenta \\
\hline$\beta$ Catenin & Dako, Denmark & $\beta$ catenin-1 & $1: 50$ & $\begin{array}{l}\text { EDTA } \\
\text { Microwave }\end{array}$ & Tonsil \\
\hline
\end{tabular}

\subsubsection{MMP-2}

Antigen retrieval was performed by immersing the test slides along with a section of normal appendix (as the positive control) in 0.01M EDTA, PH8.0 and microwaving at high power for 15 minutes. The primary monoclonal antibody was the $17 \mathrm{~B} 11$ 
clone (Novocastra Laboratories, UK) and was used at dilution 1:40. Negative controls were obtained by omitting the primary antibody.

All slides were stained on the Dako TechMate ${ }^{\mathrm{TM}} 500$ plus, using the Dako ChemMate $^{\mathrm{TM}}$ EnVision ${ }^{\mathrm{TM}}$ Detection kit and the standard operating procedure for routine immunohistochemistry.

\subsubsection{MMP-7}

No antigen retrieval was performed. A slide of breast carcinoma was used as the positive control. The primary monoclonal antibody was the clone 1D2 (Chemicon International, UK) and was used at dilution 1:25. Negative controls were obtained by omitting the primary antibody. All slides were stained on the Dako TechMate ${ }^{\mathrm{TM}} 500$ plus, using the Dako ChemMate ${ }^{\mathrm{TM}}$ EnVision $^{\mathrm{TM}}$ Detection kit and the standard operating procedure for routine immunohistochemistry.

\subsubsection{MMP-9}

Antigen retrieval was performed by immersing the test slides along with a slide of kidney (as the positive control) in 0.01M EDTA, PH8.0 and pressure-cooking for 7 minutes. The primary monoclonal antibody was the clone $15 \mathrm{~W} 2$ (Novocastra Laboratories, UK) and was used at dilution 1:80. Negative controls were obtained by omitting the primary antibody. All slides were stained on the Dako TechMate ${ }^{\mathrm{TM}} 500$ plus, using the Dako ChemMate ${ }^{\mathrm{TM}}$ EnVision $^{\mathrm{TM}}$ Detection kit and the standard operating procedure for routine immunohistochemistry. 


\subsubsection{PR}

Antigen retrieval was performed by immersing the test slides along with a slide of breast carcinoma (as the positive control) in 0.01M EDTA, PH8.0 and microwaving at high power for 15 minutes. The primary monoclonal antibody was the PgR 636 clone (Dako, Denmark) and was used at dilution 1:100. Negative controls were obtained by omitting the primary antibody. All slides were stained on the Dako TechMate $^{\mathrm{TM}} 500$ plus, using the Dako ChemMate ${ }^{\mathrm{TM}}$ EnVision $^{\mathrm{TM}}$ Detection kit and the standard operating procedure for routine immunohistochemistry.

\subsubsection{Galectin-3}

Antigen retrieval was performed by immersing the test slides along with a slide of papillary carcinoma of the thyroid (as the positive control) in 0.01M EDTA, PH8.0 and microwaving at high power for 15 minutes. The primary monoclonal antibody was the 9C4 clone (Novocastra, UK) and was used at dilution 1:150. Negative controls were obtained by omitting the primary antibody. All slides were stained on the Dako TechMate ${ }^{\mathrm{TM}} 500$ plus, using the Dako ChemMate ${ }^{\mathrm{TM}}$ EnVision ${ }^{\mathrm{TM}}$ Detection kit and the standard operating procedure for routine immunohistochemistry.

\subsubsection{CD98}

Antigen retrieval was performed by immersing the test slides along with a slide of tonsil (as the positive control) in PC citrate and microwaving at high power for 2.5 minutes. The primary polyclonal IgG antibody was the clone C-20 (Santa Cruz) and was used at dilution 1:200. A rabbit antigoat secondary antibody was used. Negative controls were obtained by omitting the primary antibody. All slides were stained on 
the Dako TechMate ${ }^{\mathrm{TM}} 500$ plus, using the $\mathrm{ABC}$-vector kit and the standard operating procedure for routine immunohistochemistry.

\subsubsection{E Cadherin}

Antigen retrieval was performed by immersing the test slides along with a slide of tonsil (as the positive control) in $0.01 \mathrm{M}$ EDTA, PH8.0 and microwaving at high power for 2.5 minutes. The primary monoclonal antibody was the NCH-38 clone (Dako, Denmark) and was used at dilution 1:25. Negative controls were obtained by omitting the primary antibody. All slides were stained on the Dako TechMate ${ }^{\mathrm{TM}} 500$ plus, using the Dako ChemMate ${ }^{\mathrm{TM}}$ EnVision $^{\mathrm{TM}}$ Detection kit and the standard operating procedure for routine immunohistochemistry.

\subsubsection{P Cadherin}

Antigen retrieval was performed by immersing the test slides along with a slide of placenta (as the positive control) in 0.01M EDTA, PH8.0 and microwaving at high power for 15 minutes. The primary monoclonal antibody was the $56 \mathrm{C} 1$ clone (Novocastra, UK) and was used at dilution 1:100. Negative controls were obtained by omitting the primary antibody. All slides were stained on the Dako TechMate ${ }^{\mathrm{TM}}$ 500 plus, using the Dako ChemMate ${ }^{\mathrm{TM}}$ EnVision ${ }^{\mathrm{TM}}$ Detection kit and the standard operating procedure for routine immunohistochemistry.

\subsubsection{1 $\beta$ Catenin}

Antigen retrieval was performed by immersing the test slides along with a slide of tonsil (as the positive control) in 0.01M EDTA, PH8.0 and microwaving at high 
power for 15 minutes. The primary monoclonal antibody was the $\beta$ catenin- 1 clone (Dako, Denmark) and was used at dilution 1:50. Negative controls were obtained by omitting the primary antibody. All slides were stained on the Dako TechMate ${ }^{\mathrm{TM}} 500$ plus, using the Dako ChemMate ${ }^{\mathrm{TM}}$ EnVision $^{\mathrm{TM}}$ Detection kit and the standard operating procedure for routine immunohistochemistry.

\subsection{Scoring}

To score P53, a similar scoring system to that used by Leversha et al was used $\{$ Leversha, 2003\}. As all cores showed homogenous staining, the intensity was scored. The intensity was classified into $0=$ no staining, $1=$ weak staining, $2=$ moderate staining and $3=$ strong staining. The positive control was used as a comparator for strong staining. As the staining pattern is known to be homogenous, the maximum score of P53 was used in calculations.

ER and PR were scored using a histoscore used in assessing expression in breast carcinoma cases. This was created by adding the intensity of staining (where $0=$ no staining, 1 = weak staining, $2=$ moderate staining and $3=$ strong staining) to the percentage of cells stained (where $0=$ no cells staining, $1=1-9 \%$ of cells staining, 2 $=10-33 \%$ cells staining, $3=33 \%-66 \%$ cells staining, $4=67-99 \%$ cells staining and $5=100 \%$ cells staining). This gave a range of scores from 0 to 8 . As both ER and PR show heterogeneous staining and both had a wide range of possible scores, the average score of the informative punch biopsies was used.

The scores for MMP-2, MMP-7, MMP-9, Galectin-3, CD98, E cadherin, P cadherin and $\beta$ catenin were assessed using a histoscore. This was calculated by multiplying the intensity of staining ( $1=$ weak, $2=$ moderate and $3=$ strong) by the percentage of 
cells showing positive staining $(1=<20 \%, 2=20-80 \%$ and $3=>80 \%)$, thus giving a score between 1 and 9. To minimise erroneous results, the average score between the sample cores' score was used in calculations.

As there is some evidence that the site of staining of Galectin-3 changes according to action of Galectin-3, the site of staining, either cytoplasmic, nuclear or cytoplasmic and nuclear was noted.

As we were expecting a decrease in E cadherin expression compared to normal endometrium, the fragments of normal endometrium within the TMAs were used as a positive control, as well as the section of tonsil. All the fragments of endometrium and epithelium in the section of tonsil showed strong, universal expression, with an overall score of 9 .

To ensure the scoring was consistent, the TMAs stained for $\mathrm{E}$ cadherin, $\mathrm{P}$ cadherin, $\beta$ catenin and ER were rescored 6 months after the initial scoring. Kappa statistics were applied to the repeat scores and showed good correlation with the results with a score of 0.63

\subsection{Statistical Analysis}

All statistical analysis was performed using SPSS 13.0 software (SPSS Inc, Chicago).

\subsubsection{Tissue Microarray}

The mean scores of the representative cores of tumour assessed for each case of USPC and EEC were calculated. The results from the main part of the tumour were used in calculations to determine if there were any differences in expression of 
antibodies between tumour types. A paired T-test was performed for the parametric results and a Mann Whitney $U$ test was performed for the non-parametric results. As multiple comparisons were being made, there is a risk that this could be interpreted as "a fishing expedition" and erroneous significant differences may be detected. Therefore a Bonferroni correction was made. This is a statistical adjustment of the $p$ value for multiple comparisons, decreasing the liklehood of erroneous false positive findings. Therefore, for Chapters 5 and7, a p value of less than 0.017 was considered significant and for Chapter 6 , a p value of less than 0.025 considered significant.

The Wilcoxon matched-pairs signed-ranks test was used to estimate relationship between staining patterns of the different antibodies used between the main part of the tumour and at the invasive edge. A p value of less than 0.05 was considered significant. The Pearson pairwise correlation coefficient was used to determine any relationship between the antibody expressions.

\subsubsection{Audit of the Pathology and Management of Uterine Papillary Serous Carcinoma (UPSC) and Correlation with Outcome (Chapter 4)}

The Spearman's Rank test was used to assess the correlation of the initial diagnostic biopsy with the definitive hysterectomy histology percentage histological subtype. Overall survival and progression free survival were estimated on the basis of KaplanMeier curves and the log-rank test used as a univariate analysis of prognostic factors. A p value of less than 0.05 was considered statistically significant, and variables that had a p value of less than 0.05 were entered into a Cox regression model. 


\section{Chapter 3}

\section{Validation of Tissue Microarray of Endometrial Carcinoma}

\subsection{Introduction}

Battifora et al \{Battifora, 1986\} introduced the multitumour tissue block in 1986 and Kononen et al \{Kononen, 1998\} refined this technique to develop the tissue microarray (TMA) in 1998. Since then the TMA has been widely used in retrospective analysis of large numbers of formalin fixed paraffin embedded tumours. The use of TMAs allows screening of up to several hundred tumours for immunohistochemistry (IHC) or fluorescent in situ hybridisation (FISH) on a single block, with a minimum outlay of reagents. As all the cores are of the same size and because all the cores are present on limited numbers of slides they can be treated identically with regards to antigen retrieval and staining thus reducing the experimental errors and discrepancies produced during analysis.

A potential shortcoming of TMA is sampling bias, whereby lack of tumour representation in the cores selected may give erroneous results, due to heterogeneity of tumours. To address this issue, the sampling of multiple cores is recommended from each case. However, there is uncertainty as to the optimal number of cores that need to be taken to give a result that is representative of the tumour as a whole. As this is likely to vary with different tumour types, it is advisable to perform a validation study from tumours arising in specific organs. Validation studies have been performed and published on a range of tumours including those from the breast, prostate, gastrointestinal tract and ovary \{Gillett, 2000;Mousses, 2002;Gulmann, 2003; Rosen, 2004\}, and have recommended between 2 and 6 cores be taken from 
each case. No tissue validation study on endometrial carcinoma has yet been published in the literature, therefore a validation study was performed to determine whether TMAs could be used in place of whole sections for IHC and, in addition, to calculate the number of cores needed from each case to ensure adequate representation of the tumour. 


\subsection{Materials and Methods}

\subsubsection{Tissues}

Archival tissue samples of 78 cases of Uterine Papillary Serous Carcinoma (UPSC) were selected from the Royal Infirmary of Edinburgh Department of Pathology, the slides were reviewed by myself and Dr A Williams (a subspecialist gynaecological pathologist), the diagnosis of UPSC was confirmed, using the criteria described in "Materials and Methods", and the appropriate blocks were selected. The H\&E sections then had at least one area from the centre of the tumour marked on the slide by the pathologist. All tissue had been routinely fixed in $4 \%$ buffered formaldehyde and processed into paraffin blocks. Forty-three of the 78 tissue blocks used in the TMA also had immunohistochemistry (IHC) performed on whole-tissue sections for comparison of the immunohistochemical staining patterns.

\subsubsection{Tissue Microarray}

The TMA was created using the technique as described in "Material and Methods". Once the TMA was made and sealed, sections were cut, with the most superficial section being stained with Haematoxylin and Eosin (H\&E) and the deepest being used for IHC for Oestrogen Receptor (ER) and P53.

\subsubsection{Immunohistochemistry}

Both ER status and P53 expression has been well documented in UPSC \{Demopoulos, 1999;Vasil'eva, 2005;Wu, 2003\}. ER expression is known to be lower in UPSC than in endometrioid endometrial carcinoma (EEC) and P53 expression is increased in UPSC compared with EEC \{Demopoulos, 1999\}. In addition ER expression tends to be heterogeneous whereas P53 expression tends to be homogenous. Therefore antibodies to ER and P53 were used to validate the TMA, 
using the techniques described in "Materials and Methods". Expression of both antibodies was assessed using the systems described in "Materials and Methods". 


\subsection{Results}

\subsubsection{Technical Efficiency of Tissue Microarray Construction}

Technical issues, including poor sealing may result in poor section quality and misalignment of cores. Misalignment of cores can result in higher cores being cut through and disappearing before other cores placed lower in the block emerge.

The H\&E, P53 and ER sections were screened and the presence of tumour was checked at each level. Table 3.1 shows a summary of the efficiency of the sections taken from the TMA. Fourteen percent of cores on the H\&E section did not show tumour and were not informative. Of these, 8 cores showed blood only, 19 showed myometrium only and 19 cores were missing. This resulted in 2 tumours not being represented by the TMA. A similar result was seen with the P53 slide with 8 cores showing blood only, 19 showed myometrium only and 23 cores were missing. This resulted in 4 cases not being represented by the TMA. Six of the ER cores showed blood only, 17 showed myometrium only and 22 cores were missing. This resulted in 8 cases not being represented.

\subsubsection{Technical Aspects of TMA Immunohistochemistry}

The percentage of informative cores was $86 \%, 85 \%$ and $86 \%$ for H\&E, P53 and ER respectively. Although there was no significant increase in core loss as the sections were taken through the TMA block, the number of cases not represented increased through the TMA. This appeared to be due to different cores cutting out the tumour at deeper levels resulting in tumour loss. Appendices 2 and 3 show the individual results of each core compared to the whole sections. 
All P53 staining was nuclear and appeared homogeneous (Figure 3.1). Table 3.2 shows the results of the sections stained with P53. Of the 74 cases with informative cores stained for P53, 54 showed complete agreement of scores between cores. In 14 cases there was a difference in score of 1,3 cases had a difference in score of 2 and one case showed a difference of 3 in the scoring (where 1 punch was negative, 3 showed a score of 2 and 4 showed a score of 3 ). Counting the score $+/-1$ as concordance, this gave an overall concordance of $92 \%$ between cores. When the scores were compressed into positive and negative the overall concordance between cores was $93 \%$.

All ER staining was nuclear and in some cases appeared heterogeneous (Figure 3.2). Table 3.2 shows the results of the ER IHC. Of the 70 cases stained with ER with informative punches, 41 either had the same score or a difference of 1 between all punches in the same case, 15 cases had a difference of 2,6 cases had a difference of 6 and 9 cases had a difference in scores of more than 4. This gave an absolute concordance of $59 \%$. When the ER scores were compressed into positive (greater than 4) or negative (less than 4), 58 cases had the same score and in 13 cases, the variation in scores between punches ranged from positive to negative. This gave an overall concordance of $82 \%$. 
Figure 3.1

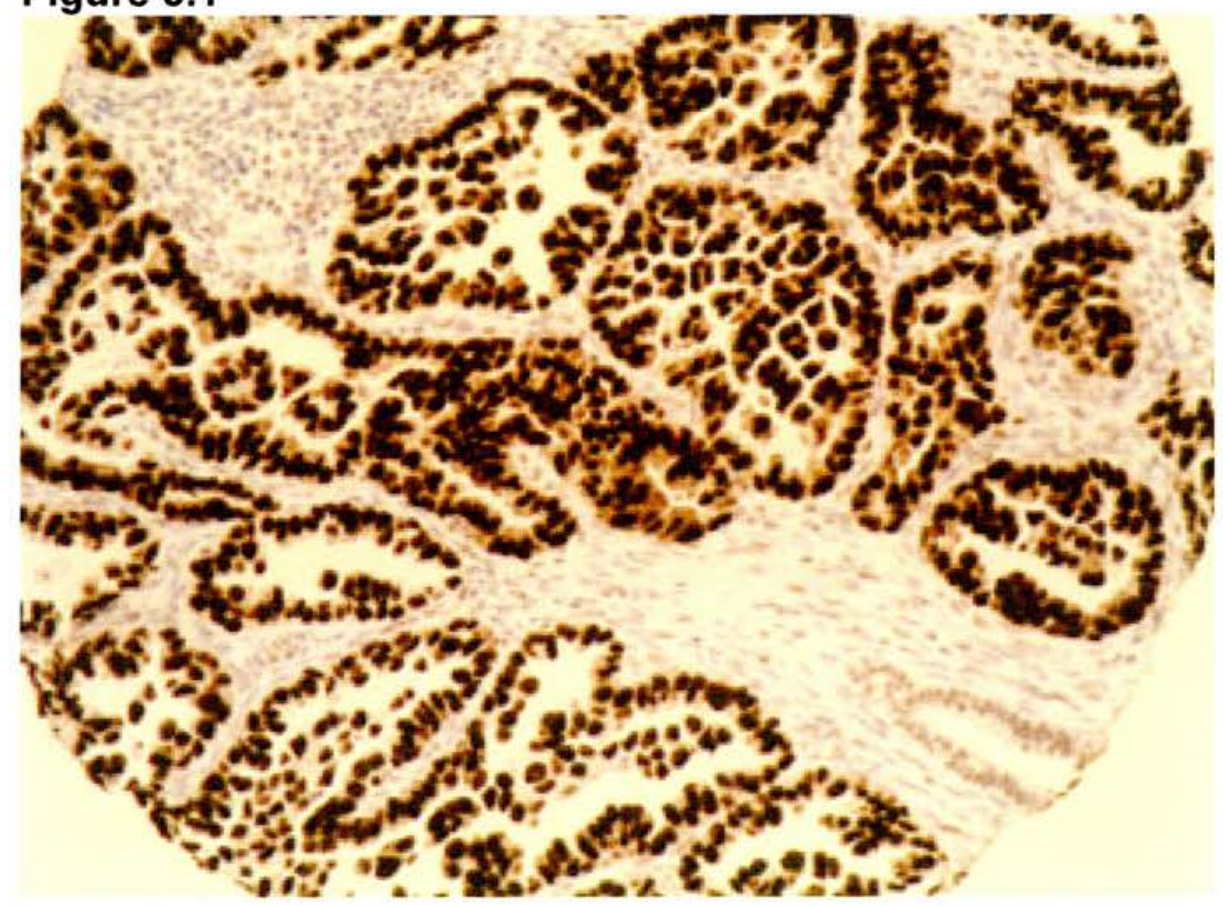

Figure 3.2

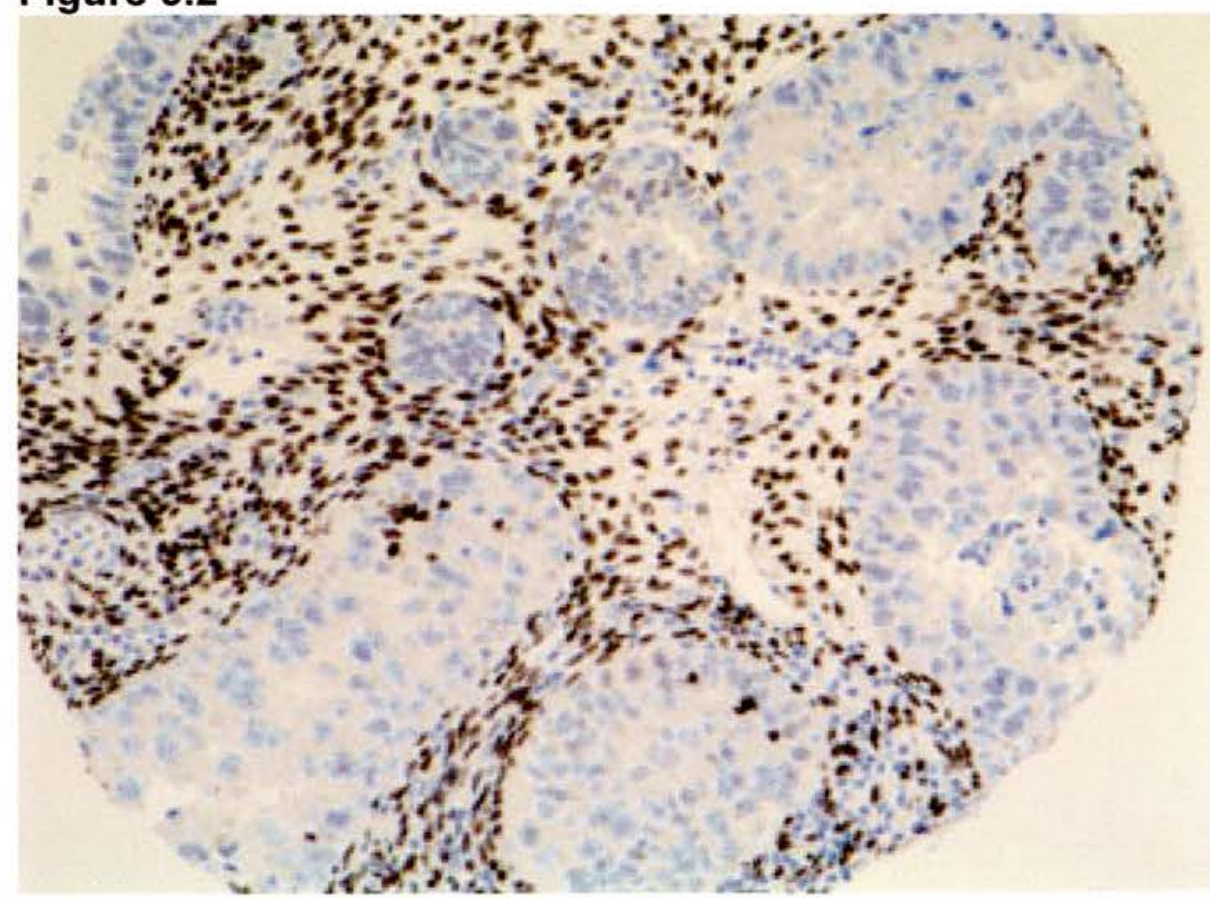

Figure 3.1. P53 Immunohistochemistry in UPSC Tissue Microarray (Magnificationx20).

Figure 3.2. ER Immunohistochemistry in UPSC Tissue Microarray (Magnificationx20). 
Table 3.1 Technical Results of TMA stained with H\&E, P53 and ER

\begin{tabular}{|c|c|c|c|c|c|c|c|c|}
\hline \multirow[t]{2}{*}{ Section } & \multirow{2}{*}{$\begin{array}{l}\text { Tumour } \\
\text { present }\end{array}$} & \multirow{2}{*}{$\begin{array}{l}\text { Tumour } \\
\text { not } \\
\text { present }\end{array}$} & \multirow{2}{*}{$\begin{array}{l}\text { Total } \\
\text { number } \\
\text { of } \\
\text { cases }\end{array}$} & \multirow{2}{*}{$\begin{array}{l}\text { Informative } \\
\text { cores }\end{array}$} & \multicolumn{3}{|c|}{ Non Informative Cores } & \multirow{2}{*}{\begin{tabular}{|l} 
Total \\
number \\
of \\
cores
\end{tabular}} \\
\hline & & & & & Blood & Myometrium & $\begin{array}{l}\text { Core } \\
\text { Missing }\end{array}$ & \\
\hline H\&E & 76 & 2 & 78 & 283 & 8 & 19 & 19 & 329 \\
\hline P53 & 74 & 4 & 78 & 279 & 8 & 19 & 23 & 329 \\
\hline ER & 70 & 8 & 78 & 284 & 6 & 17 & 22 & 329 \\
\hline
\end{tabular}




\subsubsection{Comparison of Data from Whole Sections and Microarray}

\section{Samples}

\subsubsection{P53}

Expression of P53 in the whole sections was similar to that seen in the TMA (Figure 3.3). Table 3.2 shows the results of the whole sections stained with P53. When the consensus result for the replicate punches was compared with the IHC result from the whole sections, the data were concordant for 35 of 39 tumours $(90 \%)$ for P53. In the four discordant cases the whole sections results were higher compared with the TMA results. When the data were compressed into positive and negative, 34 of the 39 tumours gave the same result, with an $87 \%$ concordance. In the 5 discordant results, in all cases, the whole sections were positive and the TMA results were negative.

Simple regression was used to determine whether the compressed or non-compressed TMA P53 score could predict the P53 score on whole sections. In both cases this showed a correlation $(\mathrm{R})$ of 0.814 and the coefficient of determination $\left(\mathrm{r}^{2}\right)$ of 0.663 . This is a large effect size and indicated that the TMA P53 score was a good predictor of the P53 score on whole sections.

\subsubsection{ER}

On examining the whole sections, most tumours revealed heterogeneous nuclear staining for the ER antibody (Figure 3.4). This was more apparent than on examination of the TMA sections. Table 3.2 shows the results of the whole sections scored with ER. The assessment of concordance was more difficult between the TMA and whole section for ER as scores ranged from 0 to 8 . However in comparing the whole section result with the TMA score, a result that was +/- 1 was deemed to 
be concordant. Therefore when the consensus result for the replicate punches was compared with the IHC result from the whole sections, the data were concordant for 22 of 36 tumours $(61 \%)$ for ER. However, when the consensus data was distributed into positive and negative we showed that 31 of 36 cases (86\%) had absolute concordance with the whole sections and in the 5 discordant cases the whole sections were positive and the TMA sections were negative (false negative results). There were no false positive results.

Simple regression was used to determine whether the TMA ER score could predict the ER score on whole sections. This showed a correlation (R) of 0.727 and the coefficient of determination $\left(\mathrm{r}^{2}\right)$ of 0.515 . This was a large effect size and indicated that the TMA ER score was a good predictor of the ER score on whole sections. When the ER results were compressed into positive and negative the correlation (R) was 0.756 and the coefficient of determination was 0.559 . This again, was a large effect size and indicated that the TMA ER score was a good predictor of the ER score on whole sections. 
Figure 3.3

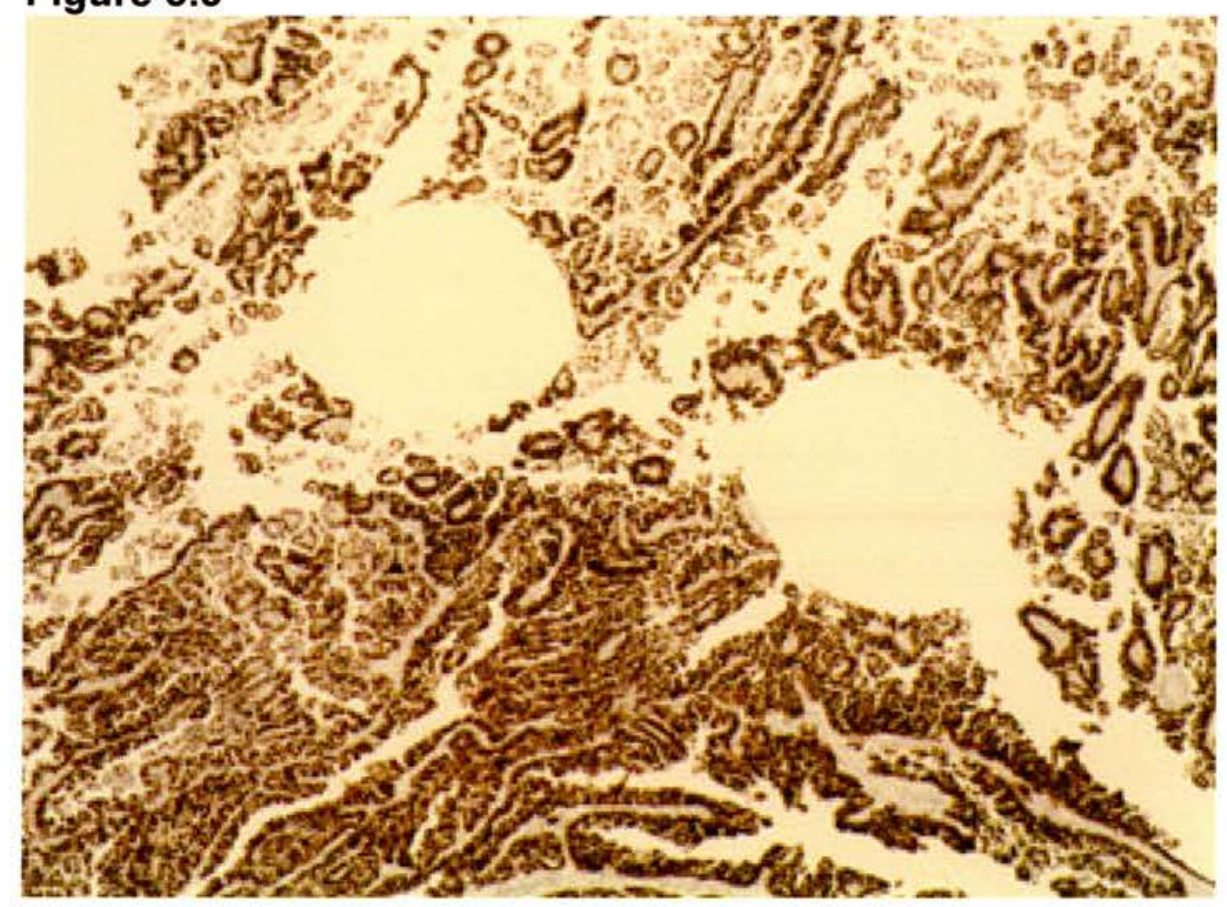

Figure 3.4

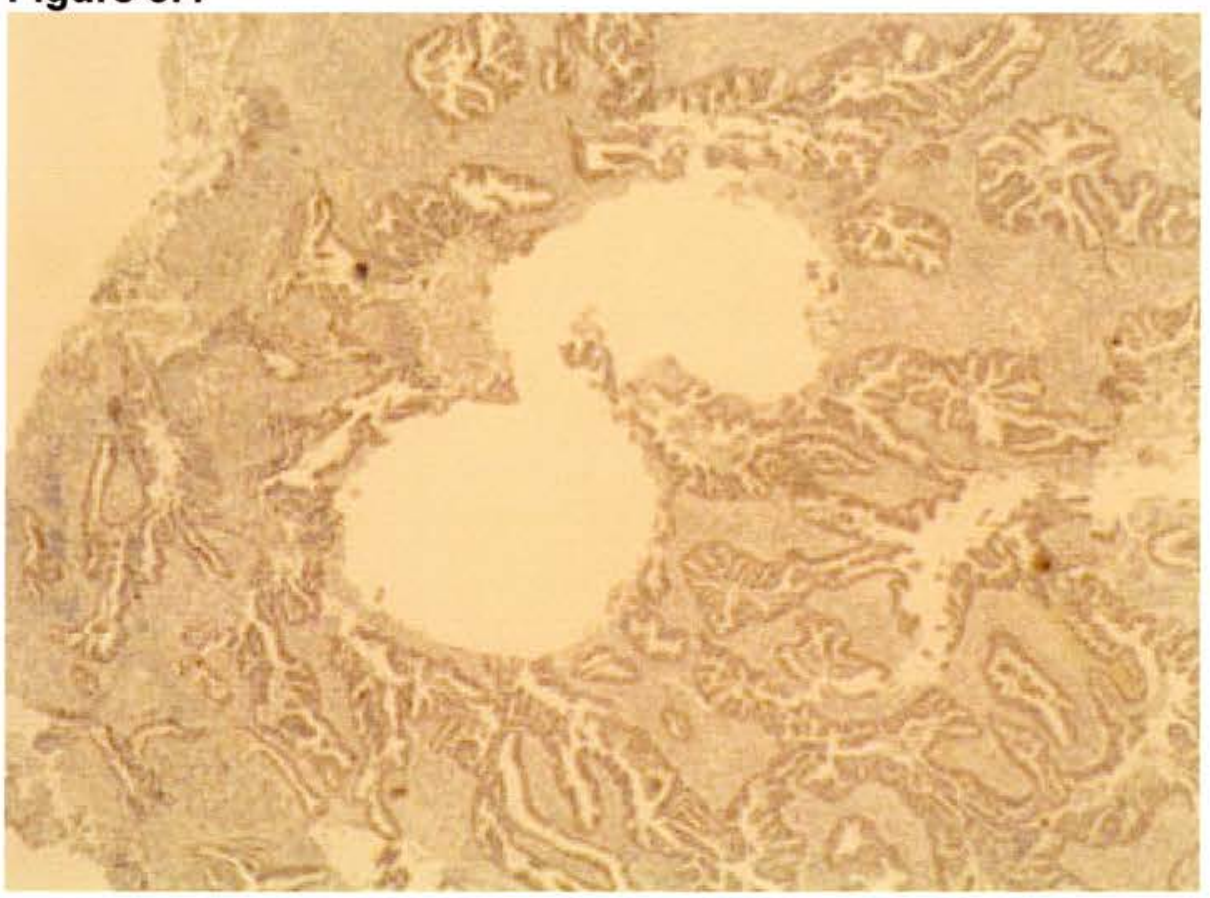

Figure 3.3. Whole section showing P53 Immunohistochemistry with hole at the Site of Core Biopsies (Magnificationx20).

Figure 3.4. Whole section showing ER Immunohistochemistry with hole at the Site of Core Biopsies (Magnificationx20). 
Table 3.2 P53 and ER Expression in TMA and Whole Sections

\begin{tabular}{|l|l|l|l|l|}
\hline \multicolumn{2}{|l|}{} & \multicolumn{2}{|l|}{ P53 } & \multicolumn{2}{l|}{ ER } \\
\cline { 2 - 5 } & Positive & Negative & Positive & Negative \\
\hline TMA number & $56(82 \%)$ & $12(18 \%)$ & $39(57 \%)$ & $29(43 \%)$ \\
$=68$ & & & & \\
\hline $\begin{array}{l}\text { Whole } \\
\text { Sections }\end{array}$ & $40(95 \%)$ & $2(5 \%)$ & $23(54 \%)$ & $19(46 \%)$ \\
$=42$ & & & & \\
\hline
\end{tabular}




\subsection{Discussion}

The objective of this chapter was to determine whether TMAs of UPSC could be used for IHC in place of whole sections. P53 and ER expression in TMAs was concordant with that of whole sections and the levels of expression in both TMAs and whole sections were generally in agreement with that in the literature \{Demopoulos, 1999;Wu, 2003;Vasil'eva, 2005\}. Agreement was higher with P53 where expression appeared more homogenous compared with ER where expression tended to be heterogeneous. It is possible that the different scoring criteria applied, with 4 categories for P53 and 8 categories for ER, also affected the level of concordance.

Leversha et al \{Leversha, 2003\} suggested that the most robust method of assessing antibody expression was to use 2 categories, either "positive" or " negative", whereas Gomaa et al \{Gomaa, 2005\} recommended splitting results into 4 groups for analysis. However, simplifying the scoring can produce erroneous results when the antibody staining pattern is heterogeneous. Compressing the ER results into positive and negative improved concordance, but there was no improvement in concordance when the P53 results were compressed. This may be due to the wider possible range of scores available for ER, whereas P53 only had 4 categories. Therefore, I feel that, where the antibody staining pattern is uncertain it is wiser to adopt a Histoscore-type scoring system, which gives a wider range of categories and thus may minimise erroneous results.

Reviewing previous validation studies, the statistical techniques used in evaluating validation have varied and have included kappa analysis, regression analysis, Pearson correlation coefficient, and re-sampling methods \{Hoos, 2001;Gillett, 
2000;Rosen, 2004;Rubin, 2002\}. I used simple regression analysis as I felt this most directly answered the research question "can TMAs be used to predict the pattern of expression of an antibody from whole sections". For both P53 and ER we demonstrated a large effect size supporting the conclusion that TMAs can be used in place of whole sections.

The second objective was to determine the optimal number of cores that needed to be taken from each case to give the most accurate results. Our informative yield was approximately $86 \%$ in sections taken from both the top and bottom of the TMA block. Punch loss occurred in $6 \%, 7 \%$ and $7 \%$ of sections stained with H\&E, P53 and ER respectively and this is similar to that in the established literature \{Leversha, 2003;Wu, 2003;Demopoulos, 1999\}. In all sections taken the majority of uninformative punches were due to sampling error where all that was present was either myometrium or blood. The punches showing myometrium only may reflect that some of the samples were taken from Stage Ia UPSC (Figure 3.5) and therefore the tumour was focal and was cut through in deeper sections or may have been taken from cases where the tumour spread in an infiltrative pattern rather than as a solid mass (Figure 3.6), thus making sampling more difficult. The punches showing blood only were cases where the tissue sampled were endometrial pipelle specimens, where often scanty fragments of tumour lie in blood and therefore these punches were prone to being cut through and the tumour was more susceptible to being missed during sampling. Overall our percentage of uninformative samples was at the higher end of the range reported in the literature (5-17\%) \{Leversha, 2003;Rosen, 2004;Fernebro, 2002;Hoos, 2001;Gillett, 2000\}, but our punch loss was similar (6-7\% compared 

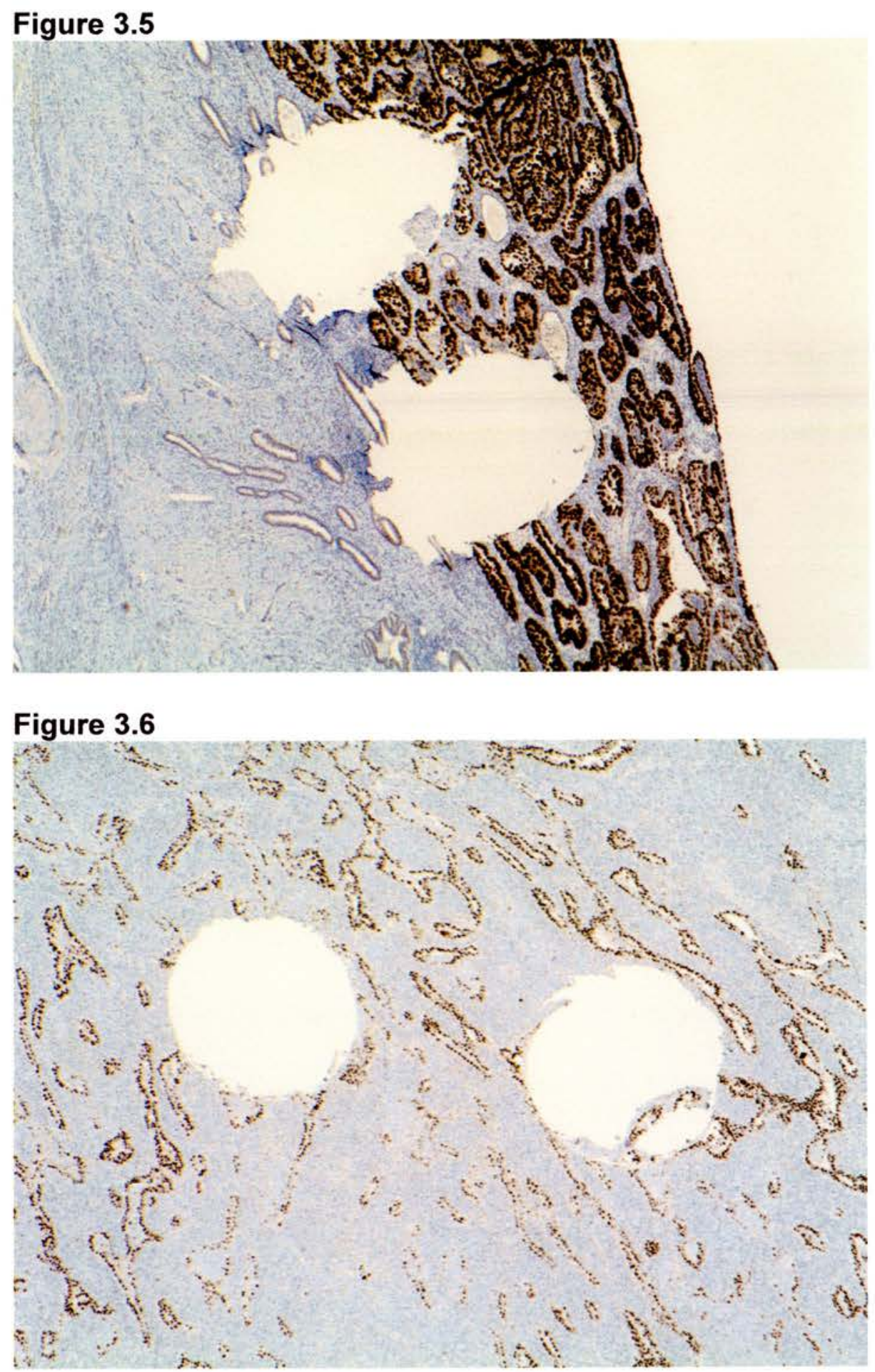

Figure 3.5. Whole Section Showing P53 Staining and Site of TMA cores in Stage 1a UPSC (Magnificationx4).

Figure 3.6. Whole Section Showing P53 Staining and Site of TMA cores in infiltrating UPSC (Magnificationx4). 
with $5 \%$ Leversha, 2003\}. The difference in informative punches in part reflects the diversity of specimens being sampled, rather than solid fragments of tumour, which are most commonly used in creating TMAs.

We found that taking more than one punch from each case increased the level of informative data from the TMA, which otherwise would have been lost. In addition, in the case of ER where expression was heterogeneous this lessened discrepancies. It is difficult to determine the exact number of punches, which should be taken from each case to ensure adequate and accurate representation, but in the case of the pipelle specimens more specimens were advisable. Torhorst et al \{Torhorst, 2001\} suggested that 4 cores was optimal for measuring Progesterone Receptor expression, but that only one core was necessary in assessing prognostic significance when the data covered more than 390 patients. However, most validation studies have shown that analysis of three $0.6 \mathrm{~mm}$ cores produces higher concordance rates than use of one core and that the use of three cores also reduces the problems associated with case loss where only 2 cores are taken \{Camp, 2000;Kallioniemi, 2001;Fernebro, 2002\}. Studies have shown difficulties assessing the significance of protein expressed at a low level in TMA and whole sections \{Leversha, 2003\}. However I did not find this to be the case where ER expression was not only low but also heterogeneous.

Whilst the UPSC TMA was validated for P53 and ER the obvious question is whether or not this validation can be extended to include all high grade endometrial carcinomas and also to the assessment of antibodies where the normal or abnormal staining patterns are uncertain. As we have demonstrated good levels of concordance with ER and P53 which not only are known to be present in low and high amounts respectively but also show heterogeneous and homogenous patterns of staining 
respectively \{Koshiyama, 1995\}, the conclusion I have drawn is that the validation results can be extended to the wider group of endometrial carcinomas and unknown antibodies.

\subsubsection{Summary}

The protein expression of P53 and ER in our TMA matched that seen in the whole sections. On balance the minimum number of cores sampled should be two and the optimal number of cores sampled should be three as this decreases variation seen in heterogeneous tumours and also increases the likelihood of an informative section in those tumours which were either focal, infiltrative or present in a pipelle specimen. In addition TMA use can be extended to include other high grade endometrial carcinomas and antibodies with uncertain patterns of expression. 


\section{Chapter 4}

\section{A Review of the Pathology and Management of Uterine Papillary Serous Carcinoma and Correlation with Outcome}

\subsection{Introduction}

While papillary morphology has been recognised in endometrial carcinoma since the turn of the last century, uterine papillary serous carcinoma (UPSC) was first described as a distinct clinical entity in 1982 by Lauchlan \{Lauchlan, 1981\} and Hendrickson et al \{Hendrickson, 1982;Hendrickson, 1982\} UPSC usually occurs in elderly, postmenopausal women and in contrast to endometrioid endometrial carcinoma (EEC), is not associated with excess oestrogen. There is conflicting data regarding the association of breast cancer with high-risk endometrial carcinomas \{Barakat, 1994;Magriples, 1993\}. It is thought that patients with breast cancer have a similar rate of low- and high-risk endometrial subtypes irrespective of tamoxifen use. However, patients with UPSC may have an increased risk of synchronous, or subsequent development of breast cancer \{Geisler, 2001;Goshen, 2000\}. This raises the possibility that UPSC and breast cancer may, in some patients, be due to the presence of mutations in cancer predisposing genes such as BRCA 1, BRCA2 or p53 \{Lavie, 2004\}.

Although accounting for only $10 \%$ of endometrial carcinomas, UPSC is an important diagnosis to make on initial biopsy since it behaves particularly aggressively, with a propensity for early metastasis which results in upstaging at the time of operation in $50-75 \%$ of clinical Stage I cancers \{Goff, 1994;Christman, 1987; Walker, 1982\}. 
This underscores the need for accurate pre-operative diagnosis, so that the surgeon can plan definitive treatment and perform accurate and thorough surgical staging by the FIGO system, including a total extrafascial hysterectomy and bilateral salpingooophorectomy, peritoneal washings, and removal of any suspicious pelvic or paraaortic lymph nodes. Although omentectomy is not in the 1988 FIGO system for staging, it is also frequently performed. This is because UPSC behaves aggressively, and has a pattern of spread similar to that of ovarian serous carcinoma. There is no evidence to date that this radical surgery affects outcome, but accurate staging may help influence current advice regarding adjuvant therapy. UPSC may exist in both 'pure' and 'mixed' forms with other endometrial cancer subtypes, in particular EEC and clear cell endometrial carcinoma (CCEC). Sherman et al \{Sherman, 1992\} states that the diagnosis of UPSC should be reserved for cancers with more than $25 \%$ UPSC pattern in the final resection specimen, although this figure appears arbitrary.

Data as to the optimum management of UPSC are limited due to its relative rarity. This audit was performed to look at the patient characteristics, histopathological characteristics and outcomes of various treatment regimens of UPSC. In particular, this study focussed on the accuracy of diagnosing UPSC on endometrial pipelle biopsies or curettings, whether the histological subtypes seen in the diagnostic biopsy correlated with those seen in the final resection specimen, and also what percentage of UPSC pattern was required to confer the poor prognostic phenotype. To date there have been several studies looking at either the oncological or surgical management of UPSC, ranging from 9 to 129 cases \{Abeler, 1990;Carcangiu, 1992;Chambers, 
1987; Christopherson, 1982;Geisler, 2001;Goff, 1994;Hendrickson, 1982;Slomovitz, 2003;Sykiotis, 2001\}. 


\subsection{Materials and Methods.}

\subsubsection{Identification of Cases.}

Cases were identified as described in "Materials and Methods".

The patient demographics, histopathology, and treatment were recorded as described in "Materials and Methods".

\subsubsection{Patient Characteristics.}

Surgical and oncological medical records were reviewed by $\operatorname{Dr}$ A Stillie (an Oncology Specialist Registrar) to obtain patients' age at presentation, any previous history of breast cancer, the treatment and follow-up data, in particular the site of any relapse. The primary endpoints were progression-free survival (PFS) and overall survival (OS), defined as the period from the date of diagnosis to the date of recurrence or the last clinic visit (if alive) or the date of death. Data were censored if information on survival was not available or the patient had ceased to be followed up for any reason but had not died of cancer.

\subsubsection{Histopathological Characteristics.}

HM and Dr D Faratian (a Specialist Registrar in Pathology) reviewed all diagnostic biopsies and hysterectomy sections from the 67 patients. The diagnosis of UPSC, clear cell carcinoma (CCEC) and EEC were made using the criteria as described in "Material and Methods". The pipelle or endometrial curettings were reviewed to determine if definitive diagnosis of UPSC was possible on the original biopsy. Then the hysterectomy specimens were reviewed to determine if it was a pure UPSC or mixed tumour. If it was a mixed tumour, the percentage of UPSC, EEC and CCEC of the total tumour present was estimated in the tissue processed for histological analysis. Estimates were made by counting low power $(\mathrm{x} 20-\mathrm{x} 40)$ fields of each 
pattern and dividing this by the total number of fields. Data were compared and the three discrepancies resolved by discussion. The number and nature of specimens additional to the hysterectomy specimen received (i.e. omentum, pelvic lymph nodes or peritoneal washings) were noted, along with the pathological stage at the time of resection using the FIGO staging criteria as described in "Materials and Methods".

\subsubsection{Oncological Characteristics}

The adjuvant therapy was recorded and the chemotherapy and radiotherapy regimens (external beam $+/$ - vaginal caesium) were documented. Where the data was available, details of the treatment of recurrence were also noted.

\subsubsection{Statistical Analysis}

Statistical analysis was performed as described in "Materials and Methods". 


\subsection{Results}

\subsubsection{Patient Characteristics}

Sixty-seven patients were identified. The median age of the patients was 68 years (range 49-89). Nine patients (13\%) had a past medical history of breast cancer. No patients developed breast cancer after the time of diagnosis of UPSC. The median follow-up for all patients was 14.5 months (range 1-114). The numbers and percentages of each patient with each stage of disease are presented in Table 4.1, along with 1 year and 3 year OS and PFS by stage. Overall survival and PFS correlated significantly with the stage of disease ( $\mathrm{p}<0.01$, Figure 4.1 ), the median OS was 67 months, 43 months, 14 months and 9 months for Stage I, II, III and IV disease, respectively. The median PFS for Stage I disease was not reached in this study, due to censoring, but the median PFS for Stage II, III and IV disease was 43 months, 9 months and 8 months respectively. 
Table 4.1. Progression Free Survival and Overall Survival by Stage

\begin{tabular}{|c|c|c|c|c|c|}
\hline \multirow{2}{*}{ Stage } & \multirow{2}{*}{$\begin{array}{l}\text { Number of } \\
\text { patients (\%) }\end{array}$} & \multicolumn{2}{|c|}{ Progression Free Survival } & \multicolumn{2}{|c|}{ Overall Survival } \\
\hline & & 1 year & 3 years & 1 year & 3 years \\
\hline Stage I & $25(37)$ & 84.7 & 68.2 & 88.2 & 64.1 \\
\hline IA & $3(4.5)$ & & & & \\
\hline IB & $14(21)$ & & & & \\
\hline IC & $8(12)$ & & & & \\
\hline Stage II & $14(20)$ & 81.8 & 60.1 & 81.8 & 50.9 \\
\hline IIA & $7(10)$ & & & & \\
\hline IIB & $7(10)$ & & & & \\
\hline Stage III & $19(28)$ & 41.7 & 25.0 & 56.2 & 21.4 \\
\hline IIIA & $14(21)$ & & & & \\
\hline IIIB & $3(4.5)$ & & & & \\
\hline IIIC & 2(3) & & & & \\
\hline Stage IV & $9(13)$ & 0 & ND & 41.6 & ND \\
\hline All Patients & 67 & 61.5 & 46.2 & 67.0 & 39.4 \\
\hline
\end{tabular}

"PFS, $\mathrm{p}<0.01$ (Kaplan-Meier); ${ }^{*} \mathrm{OS}, \mathrm{p}<0.01$ (Kaplan-Meier); $\mathrm{ND}=$ No data 
Figure 4.1. Overall Survival and Progression-Free Survival by Stage.
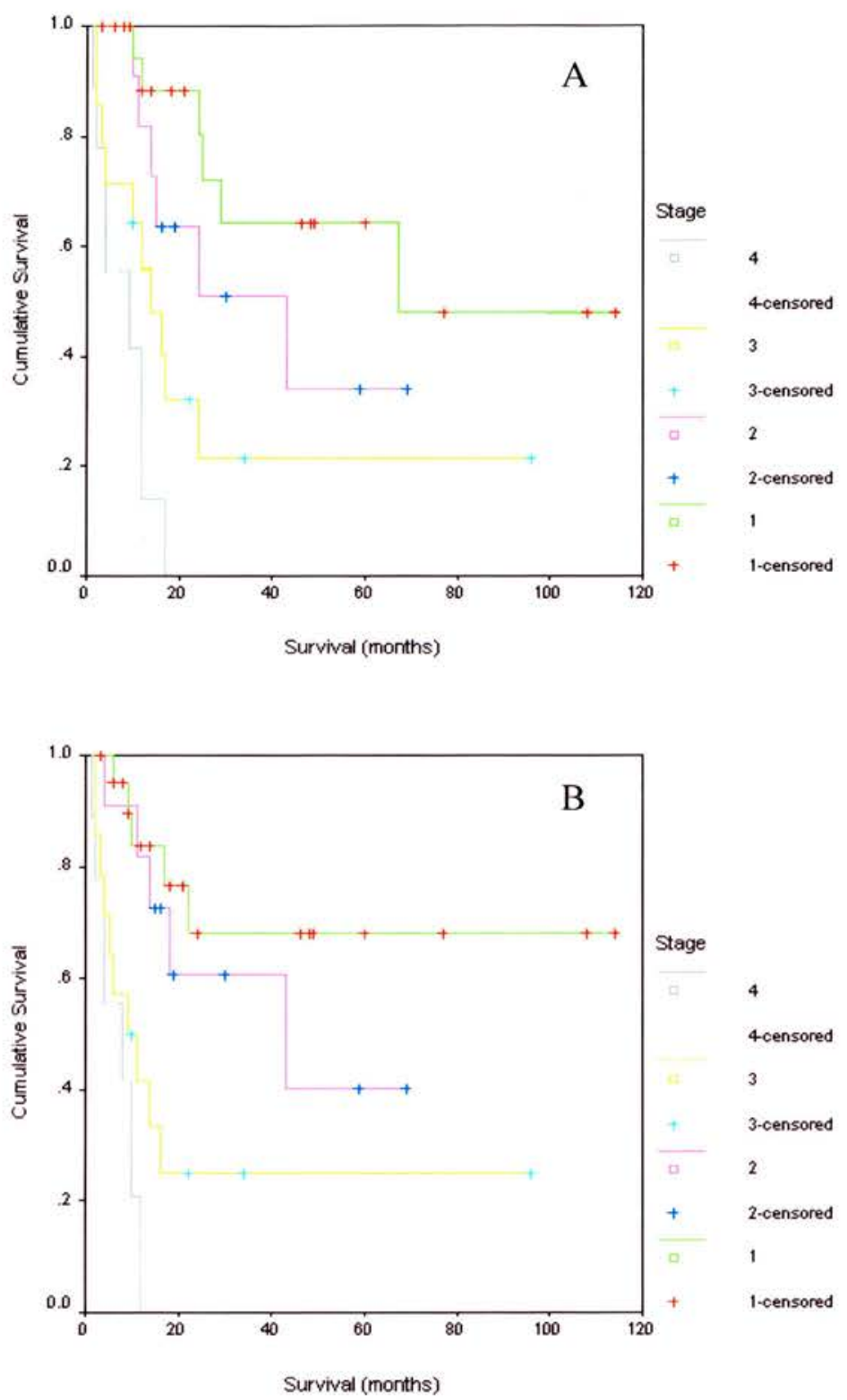

(A) Overall survival by Stage $(\mathrm{p}<0.01)$, (B) Progression-free survival by Stage $(\mathrm{p}<0.01)$. 


\subsubsection{Surgical Analysis}

Fifty-nine patients underwent surgery. All these patients had a total abdominal hysterectomy, bilateral salpingo-oophorectomy (TAH BSO). Twenty-four patients (40.1\%) had, in addition, omental sampling, of these, 21 patients $(87.5 \%)$ an omental biopsy, where only a few centimetres of omentum was removed and 3 patients $(12.5 \%)$ had full omentectomy, where all the visible omentum was removed. Seven patients $(11.9 \%)$ had a pelvic node clearance; in 30 patients $(50.9 \%)$ peritoneal washings were taken. Seven patients had TAH BSO, omentectomy, pelvic node sampling and peritoneal washings taken. No patients had para-aortic node sampling.

Seven $(29 \%)$ omental samples were positive for metastatic disease, $2(28.5 \%)$ pelvic lymph node clearances were positive, while 6 of $30(20 \%)$ of peritoneal washings contained malignant cells. Patients who had a negative omental biopsy at the time of operation had a significantly better PFS and OS $(\mathrm{p}<0.01)$ than those patients who had a positive biopsy (Figure 4.2). Of the three omentectomies performed, one was positive and two were negative. Positive peritoneal cytology was weakly associated with poor prognosis (log-rank test, $\mathrm{p}=0.15$ ), while the effect of pelvic lymphadenectomy on prognosis could not be analysed due to small numbers of patients. 
Figure 4.2. Kaplan-Meier Curves of Treatment Variables by Overall

Survival.
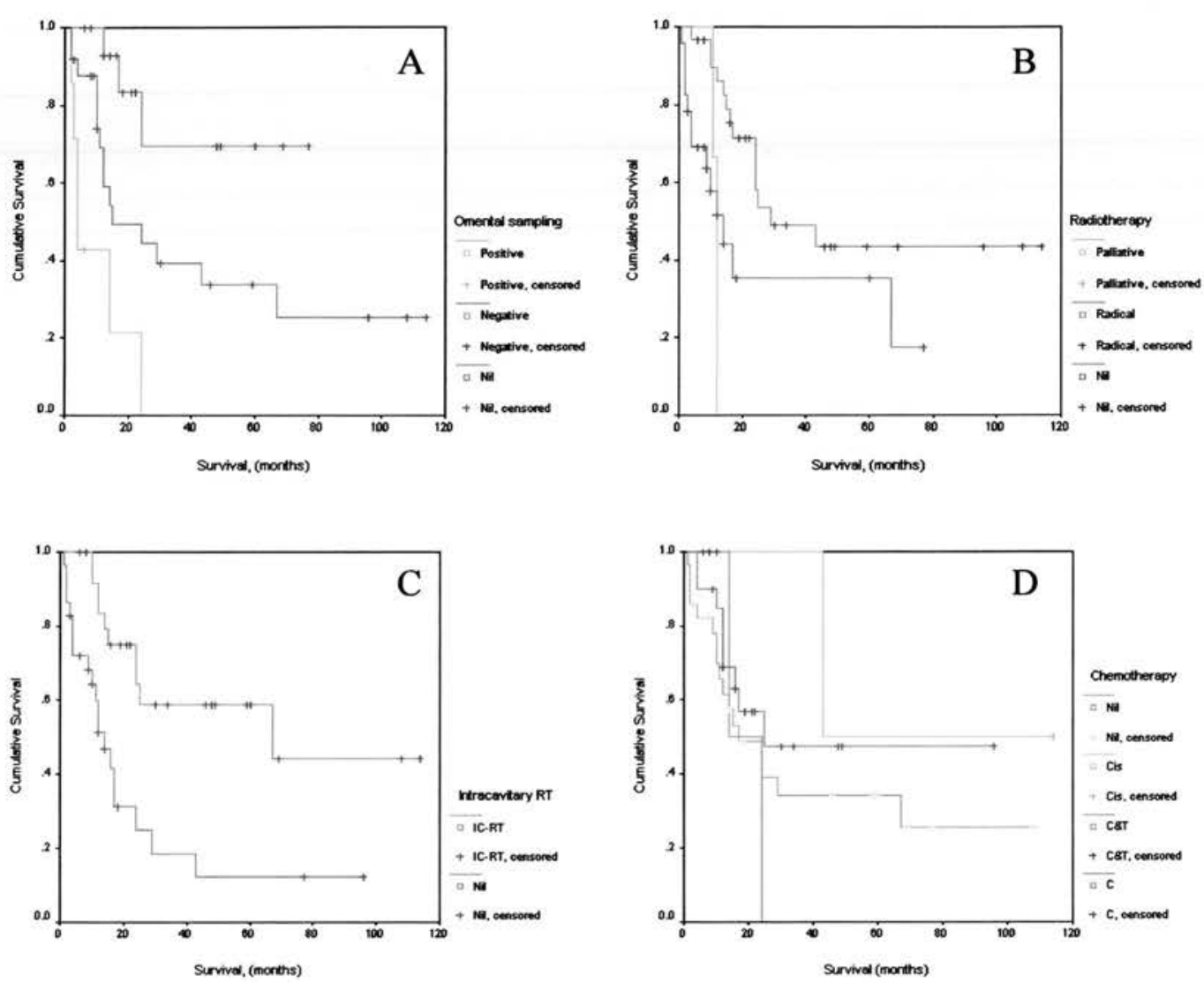

(A) Omental sampling $\mathrm{p}<0.01$ (log-rank test), (B) Radiotherapy $\mathrm{p}<0.01$ (log-rank test), (C) Intra-cavitary radiotherapy $\mathrm{p}<0.01$ (log-rank test), (D) Chemotherapy $\mathrm{p}=0.64$ (log-rank test).

Abbreviations: $\mathrm{RT}=$ radiotherapy, $\mathrm{Cis}=$ cisplatinum, $\mathrm{C} \& \mathrm{~T}=$ carboplatin and paclitaxel (Taxol), $\mathrm{C}=$ carboplatin. 


\subsubsection{Histopathological Analysis}

On reviewing the pathology reports, 63 of the cases were diagnosed by pipelle biopsy, curettage, or hysteroscopic biopsy; 1 case was diagnosed on cervical cytology, and 3 cases from hysterectomy histology. Of the 63 patients who had a preoperative diagnostic biopsy, 23 biopsies $(36.5 \%)$ showed pure UPSC histology and $39(62 \%)$ showed mixed histological subtypes including a UPSC component. Thirtynine of the diagnostic biopsies $(63 \%)$ were described as serous, papillary, mixed serous or other histological subtype in the original pathological reports, and on review 62 of the biopsies $(98.5 \%)$ contained a UPSC component. The one biopsy (1.5\%) that did not contain a UPSC component was reviewed as $100 \%$ CCEC, and on hysterectomy the tumour was reviewed as $100 \%$ UPSC. The correlation between the initial biopsy and hysterectomy histology subtypes is illustrated in Figure 4.3. While there was a weak positive correlation between the diagnostic biopsies and hysterectomy diagnoses for UPSC and CCEC (Spearman's Rank Correlation Coefficient 0.47 and 0.30 respectively, $\mathrm{p}<0.01$ ), the correlation is much stronger for EEC (Spearman's Rank Correlation Coefficient 0.70, p<0.01). In addition, those biopsies, which overestimated the amount of UPSC in the resection, were usually the same cases in which the percentage of CCEC was underestimated. In Kaplan-Meier analyses of survival, the OS and PFS were not significantly different in patients with pure and mixed histological patterns or percentage of UPSC (including less than $25 \%$ UPSC compared to more than $25 \%$ UPSC) in the diagnostic biopsies or the resections (Figure 4.4). In addition, whether UPSC was mixed with EEC or CCEC made no difference to survival. Serous endometrial intraepithelial carcinoma was identified in 2 of the hysterectomy specimens. 
Figure 4.3. Percentages of Histological Subtypes in Diagnostic Biopsies Vs Resection Specimens.

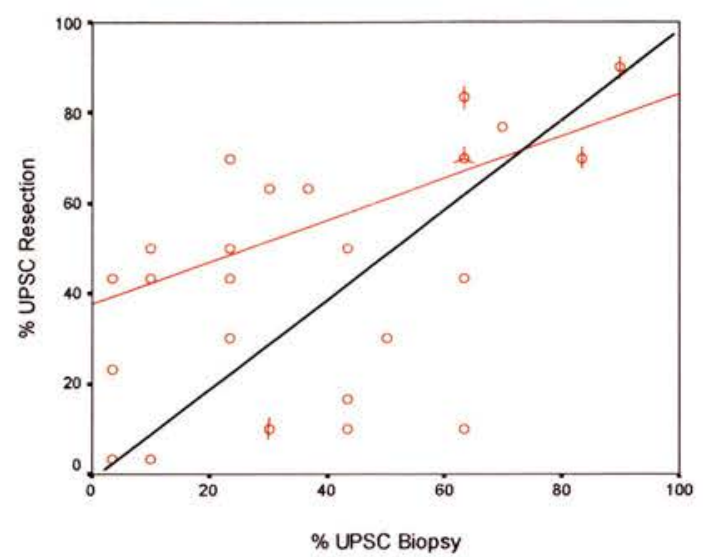

Figure 4.3. Scatterplots to represent percentages of histological subtypes in diagnostic biopsies vs resection specimens. Each spoke on a data point represents one case.

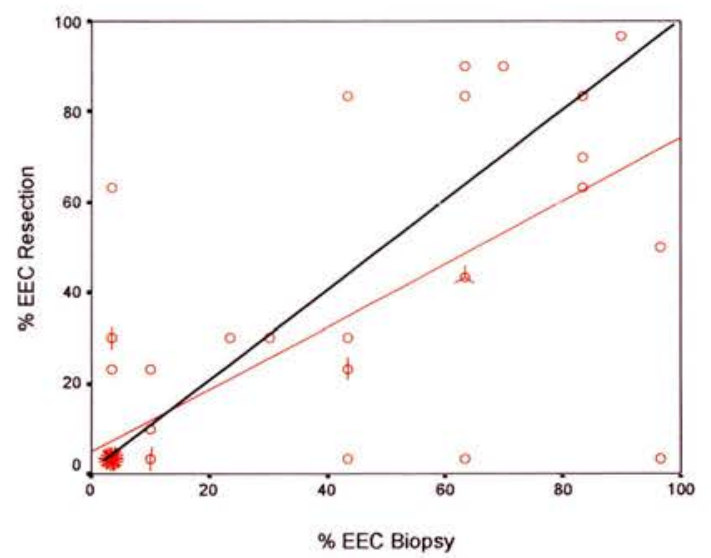

Best fit lines (red) show a positive correlation between the two specimens for all three histological subtypes.

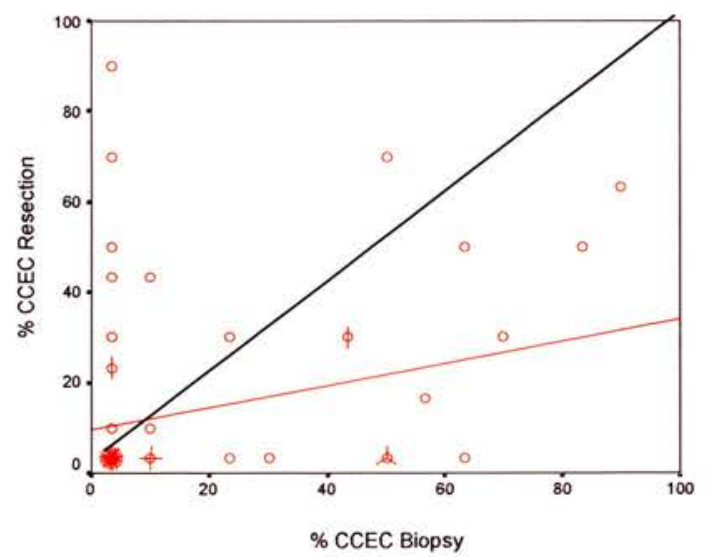


Figure 4.4. Overall Survival According to Percentage of Uterine Papillary Serous Carcinoma.
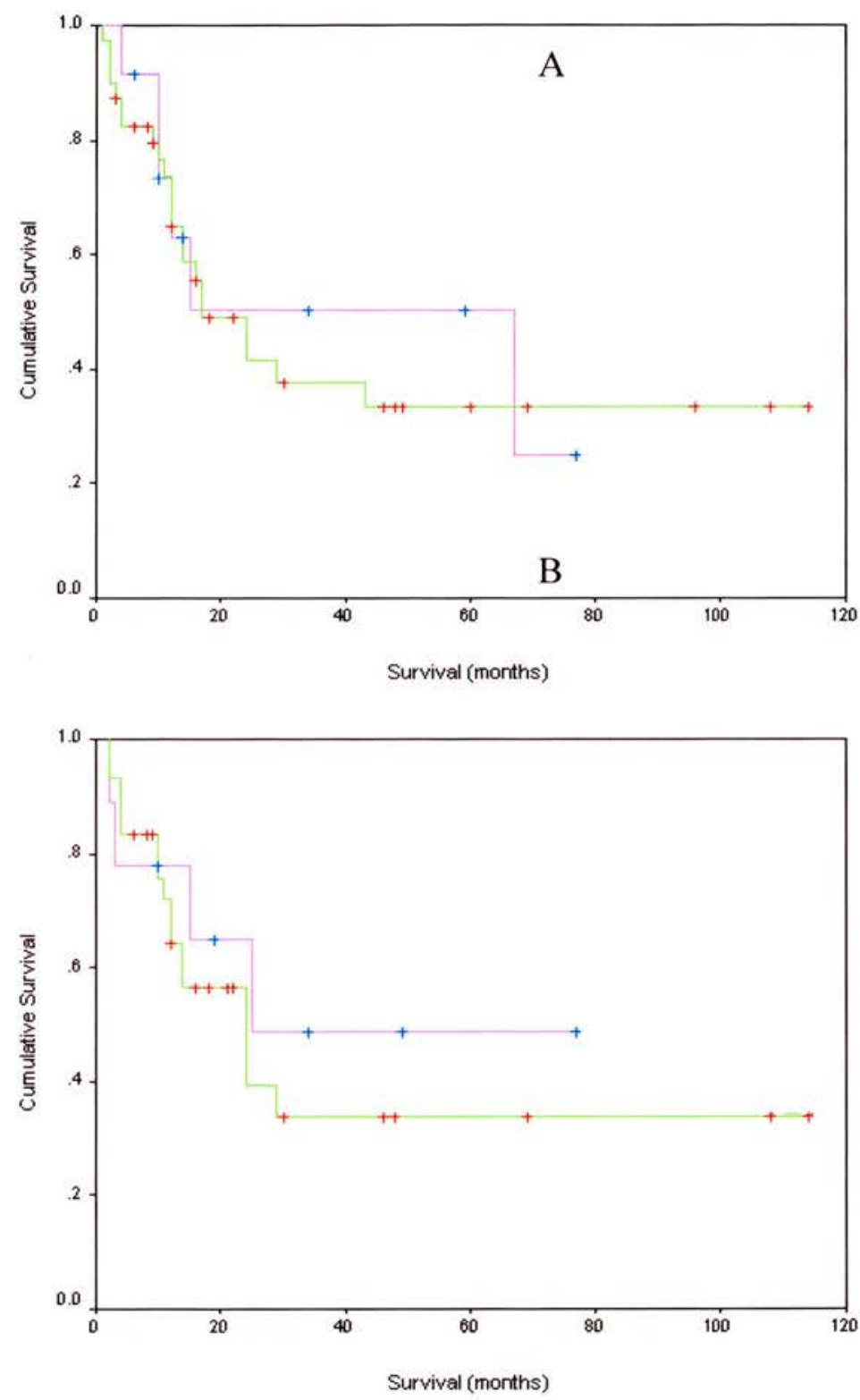

Less than $25 \%$ USPC (purple line) compared with greater than 25\% USPC (green line) in pre-operative $(\mathrm{A}, \mathrm{p}=0.76, \log$-rank test $)$ and resection specimens $(\mathrm{B}, \mathrm{p}=0.56$, $\log$-rank test). 


\subsubsection{Adjuvant therapy}

Fifty-seven patients received adjuvant therapy. Thirty patients $(52.6 \%)$ received radical radiotherapy, and 3 patients (5.2\%) palliative therapy. Of the 2 patients who did not receive radiotherapy, one was deemed to have had curative surgery (Stage I disease) and the other was deemed unfit for therapy. In addition to radiotherapy 28 patients $(49.1 \%)$ received adjuvant chemotherapy with different regimens including carboplatin alone, carboplatin and paclitaxel, or cisplatin (Table 4.2). Patients treated with radical radiotherapy postoperatively had a significantly better prognosis by univariate analysis $(\mathrm{p}<0.01$, Figure 4.2$)$. The median overall survival was 29 months in patients receiving radical radiotherapy compared with 12 months without, and the median overall survival was 67 months with palliative radiotherapy compared with 12 months without. There were no significant differences in survival in patients treated with or without chemotherapy (Figure 4.2), or between the different chemotherapy regimens.

Table 4.2. Chemotherapy Regimens by Stage.

\begin{tabular}{|l|l|l|l|l|}
\hline Stage & C & C\&T & Cis & Nil \\
\hline I & 7 & 2 & 1 & 13 \\
II & 4 & 0 & 1 & 6 \\
III & 5 & 3 & 0 & 5 \\
IV & 5 & 0 & 0 & 4 \\
\hline
\end{tabular}

$\mathrm{C}=$ carboplatin, $\mathrm{Cis}=$ cisplatinum, $\mathrm{C} \& \mathrm{~T}=$ carboplatin and paclitaxel. 


\subsubsection{Recurrence Analysis}

Fifteen $(22.4 \%)$ patients had recorded recurrences. Of these, $5(33 \%)$ were distant metastases (lung, liver, omentum and neck lymph nodes) and $10(67 \%)$ were locoregional recurrences. Median time to recurrence was 9 months (range 4-17 months). Two omental recurrences were in patients who had had positive omental biopsies. Neither had had complete omentectomy. The recurrence rate in Stage I to IV patients was $12 \%, 14 \%, 36 \%$ and $33 \%$, respectively. Further survival analysis was not possible due to the small numbers in each stage and non-standardised treatment regimens. 


\subsection{Discussion}

This study of UPSC is the third largest retrospective series of its kind with focus on the effect of histopathology on outcome. The high median age of the patients, and postmenopausal status, is similar to that previously documented for UPSC \{Dunton, 1991;Slomovitz, 2003 \}. Thirteen percent of patients had a personal history of breast cancer, identical to that observed by Slomovitz et al \{Slomovitz, 2003\} and similar to Barakat et al \{Barakat, 1994\} who observed a similar rate of low- and high-risk endometrial subtypes in breast cancer patients regardless of tamoxifen use. Following case reports and studies on Ashkenazi Jews, it was initially thought that mutations in the BRCA gene might account for this finding \{Geisler, 2001;Lavie, $2000\}$. However, a larger study by Goshen et al \{Goshen, 2000\} of 56 patients with both UPSC and breast carcinoma (not limited to Ashkenazi Jews) showed that although women with mutations in the BRCA1 gene have an increased incidence in ovarian serous papillary carcinoma and endometrial cancer, there was no specific increased risk in the development of UPSC. In addition, they failed to demonstrate mutations in either BRCA1 or BRCA2 genes in these patients. They postulated that either the BRCA mutations occurring in these patients were out with the scope of the screening techniques, or that the association between UPSC and breast cancer may be due to the presence of mutations in a variety of other cancer predisposing genes such as P53 and cerbB2.

Only $63 \%$ of the biopsies were diagnosed as pure or mixed UPSC histological patterns, in line with previous reports \{Bristow, 2001;Carcangiu, 1992;Goff, 1994;Sherman, 1992;Slomovitz, 2003;Tay, 1999;Kelly, 2005\}, which have reported the accuracy of pre-operative biopsy from $65-93 \%$. Most biopsies not explicitly 
stated as UPSC were given Grade 3 endometrial carcinoma diagnoses or classified according to the other major histological subtype, usually CCEC. However, on retrospective review of the biopsies, only one $(1.5 \%)$ of the pre-operative biopsies did not contain any UPSC, according to traditional diagnostic criteria, and the major pattern in this case was CCEC. This may be explained by the heterogeneity of the disease.

We sought to evaluate whether $25 \%$ UPSC pattern, the value above which the tumour is classified as UPSC, is prognostically valid \{Walker, 1982\}. We found that the survival of patients with less than $25 \%$ or more than $25 \%$ UPSC in the biopsy or the resection was the same, suggesting that tumours should be classified, and managed, as UPSC regardless of the percentage of UPSC in the pathological specimen. Our lowest percentage of UPSC used was 5\%. In addition, in keeping with previous reports, pure or mixed histological patterns did not show any survival difference, and survival was the same regardless of whether UPSC was mixed with a high-risk subtype, such as CCEC, or a low-risk subtype, such as EEC \{Slomovitz, 2003;Goff, 1994\}, suggesting that UPSC is biologically dominant in heterogeneous tumours.

We found a low number of patients had complete surgical staging (40\% omental staging, $11.9 \%$ pelvic node clearance, $50 \%$ cytology), which may in part be explained by the changing surgical practice over the period analysed, and difficulties with the interpretation of the diagnostic biopsies. Whilst this raises the possibility that the patients might not be accurately surgically staged, our survival rates are 
consistent with the generally accepted long-term survival rates of $35-50 \%$ for Stage I and II UPSC and 0-15\% for patients with Stage III and IV disease \{Nicklin, 1996\}.

Gehrig et al \{Gehrig, 2003\} showed that the sensitivity of a visually negative omentum was $89 \%$, concluding that, with a microscopic metastasis rate of $4 \%$, surgical sampling does not need to be included in the routine surgical staging of UPSC. The clinical consequences of under-staging is underscored by the fact that in our data two recurrences $(13 \%)$ occurred in biopsy positive omenta which were not removed by omentectomy, and these two patients had Stage III disease which, while still a poor prognosis group, may have benefited from additional abdominal surgical clearance. Omentectomy may result in better local control of disease and more directed and appropriate adjuvant therapy. Unfortunately our data were too small to establish a link between omentectomy and survival.

The rate of pelvic lymphadenectomy was low, but it is difficult to draw any conclusions from this. There has been ongoing discussion about the value of pelvic lymphadenectomy. Slomovitz et al \{Slomovitz, 2003\}, reported $19 \%$ lymph node involvement in patients without myometrial invasion and Huh et al $\{$ Huh, 2003\} demonstrated no pelvic side wall failures following observation in a population at risk for recurrence, and found in two of the patients who had not had formal pelvic lymphadenectomy, pelvic recurrence. However, the first results of the MRC-ASTEC trial, the first (and currently, the only) randomised trial investigating the role of lymphadenectomy in clinical Stage 1 endometrial carcinoma have shown that there was no benefit of lymphadenectomy, with 3-year overall survival rates of $89 \%$ (TAH BSO alone) and $88 \%$ (TAH, BSO and lymphadenectomy). In addition the 3-year 
recurrence free survival was also better in the TAH BSO alone arm and relapse rates were similar (H Kitchener, oral presentation at the European Society for Gynecologic Oncology, Istanbul, September 2005).

We did not show any significant impact of adjuvant radiotherapy or chemotherapy on PFS or OS. Ramondetta et al \{Ramondetta, 2001\} showed that single agent paclitaxel showed a tumour response in $77 \%$ of patients, and used in a neo-adjuvant fashion with cisplatin, Zanotti et al \{Zanotti, 1999\} saw responses in 8 out of 9 patients. In addition Kelly et al $\{$ Kelly, 2005\} recently showed that platinum based chemotherapy improved disease free and overall survival of patients with Stage I UPSC and vaginal cuff radiation provided local control of disease. However, our study failed to show that treatment with these agents converted into an objective improvement in PFS or OS. This is probably due to the short average follow up time (14 months) and possibly the inadequate surgical staging, resulting in patients being understaged.

Twenty-two percent of patients in this study had further disease. This is far fewer than the percentage observed by other groups, who have documented recurrence rates of between $50-75 \%$, using various adjuvant therapies, radiotherapy alone or with the addition of chemotherapy using platinum and/or paclitaxel alone, as used in our study \{Sood, 2003;Zanotti, 1999;Mallipeddi, 1993;Frank, 1991\}. Our results therefore compare favourably with similarly staged tumours at equivalent median follow-up, although similar to these studies Stage III and IV tumours remain a particular management problem due to higher rates of recurrence $(36 \%$ and $33 \%$ for 
Stage III and IV carcinomas compared with $12 \%$ and $14 \%$ for Stage I and II carcinomas, respectively). Establishing the exact reasons for favourable recurrence rates is hampered by limited patient numbers and inconsistent and non-randomised adjuvant therapies, but it appears that surgery produces good regional control in this group, with $67 \%$ locoregional control compared to the $87 \%$ observed by Sood et al \{Sood, 2003\}.

\subsubsection{Summary}

Despite having been described as a distinct clinical entity for over twenty years, UPSC remains a management challenge for the multidisciplinary team due to the paucity of understanding on the epidemiology, pathogenesis, natural history and optimal treatment strategies for this important and aggressive variant of endometrial adenocarcinoma. These data contribute to the growing body of literature on UPSC, and addresses diagnostic and treatment uncertainties for the pathology, surgical and oncological teams. This study raised awareness for the need of accurate and complete surgical staging at a local level. However, we have not altered our approach to the use of adjuvant chemoradiotherapy for patients with UPSC as our patient numbers were small with a limited follow up time. This study contributes to the increasing local, national and international awareness of the need to invest in randomised clinical research trials on UPSC, at a time where alternative treatment modalities may become increasingly effective. 


\section{Chapter 5}

\section{The Role of E Cadherin, P Cadherin and $\beta$ Catenin in Uterine Papillary Serous Carcinoma and Endometrioid Endometrial Carcinoma.}

\subsection{Introduction}

This chapter focuses on the first step on invasion; namely detachment of tumour cells from each other. Normal cell-cell adhesion determines the morphology of a tissue type and regulates major cellular processes including motility, growth differentiation and survival. Cadherins are a widely distributed family of cell-cell adhesion molecules which are composed of 3 parts, a single domain transmembrane portion, which, using calcium, binds to another transmembrane domain of a cadherin molecule and a cytoplasmic portion composed of 2 domains \{Nose, 1988; Matsuzaki, $1990\}$. The cytoplasmic domain is connected to $\beta$ - and $\gamma$-catenins which in turn bind to the actin cytoskeleton through $\alpha$ catenin $\{$ Nathke, 1994;Hinck, 1994;Jou, 1995\}. The family of cadherins currently has more than 80 members including the classical cadherins, desmogleins, desmocollins and protocadherins. The most completely described cadherins are the classical types, Epithelial (E)-, Placental (P)- and Neuronal $(\mathrm{N})$ - cadherin and all vertebrate cells seem to express at least one cadherin \{Niessen, 2002; Takeichi, 1991\}. Cadherin-catenin complexes also have roles in intercellular communication and modulation of cell function in both normal and malignant tissues. 


\subsubsection{E Cadherin}

$\mathrm{E}$ cadherin is the most common cadherin in epithelial cells. It is made from $4.5 \mathrm{~kb}$ mRNA as a $135 \mathrm{kDa}$ precursor polypeptide, which is processed rapidly by proteolytic cleavage to the mature $120 \mathrm{kDa}$ form \{Shore, 1991\}. The E cadherin-catenin complex is necessary for cell-cell adhesion of epithelial cells. Failure to assemble the E cadherin-catenin complex or to properly connect to the actin cytoskeleton results in loss of cell adhesion, which may be involved in tumour spread \{Van Roy, 1992;Birchmeier, 1993;Guilford, 1998;Perl, 1998\}. The E cadherin gene (CDH1) is found on chromosome 16q22.1. CDH1 is thought to be a tumour suppressor gene, whose loss has been shown to cause tumour invasion and metastasis in various cancer models \{Christofori, 1999\}. In endometrial endometrioid carcinoma (EEC) loss of heterozygosity at $16 \mathrm{q} 22$ has been related to poor prognosis and CDH1 mutations and promoter hypermethylation have been found in a small percentage of cases \{Kihana, 1996;Risinger, 1994;Moreno-Bueno, 2003;Saito, 2003\}. In addition to abnormalities in promoters of the $\mathrm{E}$ cadherin gene, or the gene itself, the $\mathrm{E}$ cadherin-catenin complex can be disturbed by the action of TGF- $\beta$. Vogelmann et al $\{$ Vogelmann, 2005\} demonstrated that TGF- $\beta$ induced destabilisation of the $\mathrm{E}$ cadherin-catenin complex and thus dissociation of $\beta$ catenin from $\alpha$ catenin and the actin cytoskeleton. Somatic mutations in the TGF- $\beta$ signalling pathway are associated with loss of proliferative control, malignant progression, invasion and metastasis \{Vogelmann, 2005\}. They also demonstrated that the mechanism involved phosphorylation of $\beta$ catenin by PTEN \{Vogelmann, 2005\}, 
E cadherin is present in endometrial epithelium in proliferative phase and decreases in secretory phase $\{$ Shih, 2004\}. It is possible that the cadherin-catenin complex may have a role in the maintenance of the normal endometrial architecture.

There is decreased expression of E cadherin in atypical hyperplasia compared to normal endometrium and even less in endometrial carcinoma \{Shih, 2004;MorenoBueno, 2003\}. Studies have also shown that loss of E cadherin expression increases with increasing grade of the endometrial carcinoma, such that the most poorly differentiated tumours have the least E cadherin expression \{Moreno-Bueno, 2003;Shih, 2004;Dvalishvili, 2005\}. Some studies have shown that E cadherin loss is more frequent in UPSC and clear cell carcinoma, compared to EEC \{Holcomb, 2002;Moreno-Bueno, 2003\}. In contrast, Demopoulos et al \{Demopoulos, 1999\} did not find any differential expression of $\mathrm{E}$ cadherin between EEC and UPSC. However, this study examined a relatively small number of tumours (14 cases of EEC and 10 cases of UPSC).

E cadherin negative endometrial tumours are more likely to be poorly differentiated, with local and distant dissemination of disease \{Pijnenborg, 2004;Leblanc, 2001;Sakuragi, 1994;Holcomb, 2002\}. Shih et al \{Shih, 2004\} found that nuclear $\beta$ catenin expression was inversely correlated with E cadherin expression. However, Moreno-Bueno et al \{Moreno-Bueno, 2003\} did not find any link between reduced E cadherin expression and nuclear accumulation of $\beta$ catenin.

As $\mathrm{E}$ cadherin has roles in cell-cell adhesion it is possible that the differential expression in E cadherin between EEC and UPSC may be, in part responsible for the poor prognosis of UPSC and increased propensity for myometrial and distant spread. 


\subsubsection{P Cadherin}

P cadherin is expressed in trophoblast and shows restricted expression in epithelia, being expressed predominantly in basal cells of bronchial and mammary epithelium \{Smythe, 1999;Deugnier, 1999\}. This basal expression suggests that $\mathrm{P}$ cadherin may not only have a role in cell-cell adhesion, but also may have a role in differentiation and cell growth. Studies on pancreatic cell lines demonstrated that over expression of P cadherin caused increased motility of cells and accumulation of cytoplasmic p120ctn, a protein involved in cell-cell adhesion which acts by stabilising cadherin and regulating cadherin turnover \{Taniuchi, 2005; Kowalczyk, 2004\}.

Although $\mathrm{P}$ cadherin is similar to $\mathrm{E}$ cadherin the pattern of expression in malignancy and in particular in both normal and malignant endometrium is less well defined. An immunohistochemical study by Van Der Linden et al \{Van der Linden, 1994\} showed similar staining characteristics of $\mathrm{P}$ cadherin to $\mathrm{E}$ cadherin in normal endometrium and they suggested that $\mathrm{P}$ cadherin might play a role in maintenance of proliferative endometrial glands. $\mathrm{P}$ cadherin up regulation has been demonstrated in inflammatory bowel diseases and some neoplasias such as breast and cervical adenocarcinomas \{Sanders, 2000; Palacios, 1995; Han, 2000\}. In addition up regulation of $\mathrm{P}$ cadherin has been found, in increasing frequency, in atypical endometrial hyperplasia, EEC and in a group of "non EEC", which included UPSC and clear cell carcinoma \{Moreno-Bueno, 2003\}. Interestingly in breast and cervical adenocarcinoma and "non EEC" increased P cadherin expression is related to cell proliferation, dedifferentiation, and negative oestrogen and progesterone receptors, and in addition, increased $\mathrm{P}$ cadherin has been shown to confer poor prognosis in 
breast cancer $\{$ Han, 2000;Gamallo, 2001\}. In contrast decreased expression of $\mathrm{P}$ cadherin has been described in both oral squamous cell carcinoma and melanoma. In addition $\mathrm{P}$ cadherin loss is associated with poor prognosis in oral squamous cell carcinoma $\{$ Lo, 2005\}. Recent studies have shown that a truncated form of the $\mathrm{N}$ terminal part of $\mathrm{P}$ cadherin is expressed by human melanoma cell lines and tissue from melanomas, but not by normal melanocytes or keratinocytes \{Bauer, 2005; Hoek, 2004\}. In addition, analysis showed that melanoma cells, rather than binding this short form of $\mathrm{P}$ cadherin to the cell membrane, which classically occurs in melanocytes, secreted it. The secreted form of $\mathrm{P}$ cadherin was shown to regulate the homophilic interaction between cadherin molecules by antagonising their homophilic binding, acting as a dominant negative form to interrupt cell-cell attachment. In addition to the shift from the membranous form to the secreted form expression of $\mathrm{P}$ cadherin is down regulated in malignant melanoma $\{$ Hoek, 2004\}.

\subsection{3 $\beta$ Catenin}

$\beta$ catenin has roles in both cell-cell adhesion and intracellular signalling. It controls $\mathrm{E}$ cadherin mediated cell adhesion at the plasma membrane and mediates the interplay of adherens junction molecules with the actin cytoskeleton. In addition, $\beta$ catenin acts in the Wnt signalling pathway, activating the transcription of target genes responsible for cellular proliferation and differentiation, along with LEF/TCF (lymphoid enhancer factor/T-cell factor) DNA binding proteins \{Morin, 1997; Rubinfeld, 1997\}. The signalling function of $\beta$ catenin is mainly regulated by altering its stability and Wnt signalling induces the stabilisation of the free cytoplasmic pool of $\beta$ catenin \{Polakis, 2000\}. In the absence of Wnt signalling, $\beta$ 
catenin is rapidly degraded by a destruction complex consisting of adenomatous polyposis coli (APC), axin, glycogen synthase kinase beta, conductin and casein kinase \{Behrens, 1998;Kishida, 1998;Liu, 2002\}.

Both functions of $\beta$ catenin are deregulated in malignancy leading to the loss of both cell-cell adhesion and to the increased transcription of Wnt target genes. Mutations that activate the Wnt- $\beta$ catenin pathway promote stabilisation of $\beta$ catenin and induce its nuclear accumulation \{Munemitsu, 1995\}. This results in activated gene transcription, altered cell migration and cell polarity. Inactivating mutations in the APC gene product, a protein that promotes degradation of cytoplasmic $\beta$ catenin, leads to an accumulation of cytoplasmic $\beta$ catenin \{Munemitsu, 1995;Morin, 1997;Rubinfeld, 1997\}. However, neither LOH nor promoter hypermethylation of APC is associated with nuclear expression of $\beta$ catenin $\{$ Koppert, 2004\}. Other mutations that decrease the degradation of $\beta$ catenin include those that affect the function of conductin or axin $\{\mathrm{Liu}, 2000 ;$ Satoh, 2000;Jin, 2003\}.

$\beta$ catenin co-localizes with $\mathrm{E}$ cadherin and $\gamma$ catenin at the cell membrane in all phases of the menstrual cycle $\{$ Nei, 1999;Miyamoto, 2000\}. In addition, nuclear $\beta$ catenin expression has been observed in the proliferative phase, suggesting a role for the Wnt signalling pathway in the normal endometrium \{Miyamoto, 2000\}. Chen et al $\{$ Chen, 1998\} demonstrated that progesterone, but not $\beta$ oestradiol increased $\beta$ catenin mRNA levels in cultured human endometrial stromal cells and this finding is supported by Fujimoto et al \{Fujimoto, 1998\} who demonstrated that $\beta$ catenin and $\mathrm{E}$ cadherin levels were less in the proliferative phase, where progesterone levels are 
comparatively higher compared to the secretory phase, where oestrogen levels are comparatively higher.

The activation of the Wnt signalling pathway due to $\beta$ catenin mutations has been implicated in the development of some endometrial carcinomas \{Kariola, 2005\}. Moreno-Bueno et al \{Moreno-Bueno, 2004\} showed that nuclear $\beta$ catenin expression was found in $31 \%$ of EECs and 3\% of "non EECs". However, up to $25 \%$ of endometrial carcinomas have $\beta$ catenin nuclear accumulation without evidence of $\beta$ catenin mutations, suggesting alterations in other molecules that can modulate the Wnt pathway such as APC, $\gamma$ catenin, and axin $\{$ Moreno-Bueno, 2004\}. Pijnenborg et al \{Pijnenborg, 2004\} found that nuclear localization of $\beta$ catenin, associated with mutations in the $\beta$ catenin gene and mutations in the APC gene are not predictive for recurrent disease.

In vitro studies on breast cell lines have shown that decreased $\mathrm{E}$ cadherin can augment $\beta$ catenin oncogenic signalling \{Yang, 2001\} and Shih et al $\{$ Shih, 2004\} demonstrated an inverse correlation between nuclear $\beta$ catenin and $\mathrm{E}$ cadherin expression in normal and malignant endometrium. However, Moreno-Bueno et al \{Moreno-Bueno, 2003\} did not find any link between reduced E cadherin expression and nuclear accumulation of $\beta$ catenin.

Abnormal expression of $\mathrm{E}$ cadherin and $\alpha-, \beta-$, and $\gamma$-catenins has been shown to be associated with poor prognosis in many malignancies, including endometrial and ovarian cancer \{Bremnes, 2002;Lim, 2002;Umbas, 1994;Zhao, 2003;Bringuier, 1993;Ropponen, 1999;Aaltomaa, 1999;Bohm, 2000;Zhou, 2002;Lee, 2002;Pantel, 1998;Shimazui, 1997;Endo, 2000;Voutilainen, 2006\}. 


\subsubsection{Summary}

Previous studies examining the expression of $\mathrm{E}$ cadherin, $\mathrm{P}$ cadherin and $\beta$ catenin have concentrated on EEC and its precursors and in those papers which have also looked at UPSC, the numbers of cases have been small, or they have been incorporated into a "non EEC" group alongside other carcinomas such as clear cell carcinoma. Whilst Holcomb and Moreno-Bueno et al \{Moreno-Bueno, 2003;Holcomb, 2002\} showed that E cadherin expression was lower in "non EEC" compared to EEC, Demopoulos et al \{Demopoulos, 1999\} failed to make this distinction. Therefore this chapter aims to clarify whether or not E cadherin expression is lower in UPSC compared with EEC and also to determine if there is any differential expression of $\mathrm{E}$ cadherin between the central viable part of the tumour and at the invasive edge.

The expression and role of $\mathrm{P}$ cadherin in endometrial carcinomas is unclear, although some studies have proposed that increased expression of $\mathrm{P}$ cadherin may be related to increased cell proliferation, dedifferentiation and negative ER and PR, and in the case of breast cancer may be associated with poor prognosis. This chapter aims to determine whether or not P cadherin is upregulated in UPSC compared with EEC and also if there is differential expression of $\mathrm{P}$ cadherin between the central viable part of the tumour and at the invasive edge. As $\beta$ catenin forms complexes with both $\mathrm{E}$ cadherin and $\mathrm{P}$ cadherin as part of its role in cell-cell adhesion, its expression was also studied in order to determine if there was differential expression between UPSC and EEC, whether or not there was an inverse correlation between $\mathrm{E}$ cadherin expression and $\beta$ catenin expression and also to determine if there was any 
difference in expression of $\beta$ catenin between the viable central part of the tumour and at the invasive edge. 


\subsection{Materials and Methods}

\subsubsection{Tissues}

Tissues were obtained as described in "Materials and Methods", Section 2.1.

\subsubsection{Tissue Microarray}

The tissue microarray was constructed as described in "Materials and Methods", Section 2.2.

\subsubsection{Immunohistochemistry}

Immunohistochemistry was performed for $\mathrm{E}$ cadherin, $\mathrm{P}$ cadherin and $\beta$ catenin using the technique described in "Materials and Methods", Sections 2.3.9, 2.3.10 and 2.3.11 respectively.

\subsubsection{Scoring}

Scoring of antibody expression was assessed using the technique as described in "Materials and Methods", Section 2.4.

\subsubsection{Statistical Analysis}

Statistical analysis was performed as described in "Materials and Methods", Section 2.5 . 


\subsection{Results}

\subsubsection{Intertumoural Variability}

E cadherin expression in both EEC and UPSC was significantly lower than that in the normal endometrial epithelium where the score for expression was uniformly 9 (p values $<0.00$ for both tumour types, Independent $T$ Test, Figure 5.4). Significantly greater E Cadherin expression was present in EEC compared to UPSC ( $p=0.03$ Independent $\mathrm{T}$ Test, Table 5.1) and significantly less expression of $\mathrm{P}$ cadherin was noted in EEC compared to UPSC ( $\mathrm{p}=0.013$ Independent $\mathrm{T}$ Test, Table 5.1). There was no significant difference in $\beta$ catenin expression between UPSC and EEC. Scatterplots and Pearson pairwise correlation coefficient showed a direct correlation between $\beta$ catenin immunohistochemical staining and $\mathrm{E}-$ and $\mathrm{P}$ cadherin expression for both EEC and UPSC. Of the UPSC cases, correlation between $\beta$ catenin and P cadherin showed a correlation coefficient of 0.685 and a p value of $<0.000$, and between $\beta$ catenin and $E$ cadherin showed a correlation coefficient of 0.389 and a $p$ value of 0.001 (Pearson Pairwise Correlation Coefficient).

Of the EEC cases, correlation between $\beta$ catenin and $\mathrm{P}$ cadherin showed a correlation coefficient of 0.705 and a $\mathrm{p}$ value of $0.001, \beta$ catenin and $\mathrm{E}$ cadherin immunohistochemistry showed a correlation coefficient of 0.767 and a p value of $<0.000$ (Pearson Pairwise Correlation Coefficient). 
Table 5.1. Expression of $E$ Cadherin, $P$ Cadherin and $\beta$ Catenin in the Central Part of the Tumour in UPSC and EEC.

\begin{tabular}{|l|c|c|}
\hline Antibody & UPSC & EEC \\
\hline E Cadherin & 7.06 & 7.41 \\
\hline P Cadherin & SD 1.69 & SD 2.91 \\
& ${ }^{* *} 7.53$ & 7.24 \\
$\beta$ Catenin & SD 1.9 & SD 3.1 \\
& 7.04 & 7.78 \\
& SD 2.0 & SD 1.7 \\
\hline
\end{tabular}

$\mathrm{SD}=$ Standard Deviation

*P $=0.03$ (Independent T Test)

${ }^{* *} \mathrm{P}=0.013$ (Independent T Test) 


\subsubsection{Intratumoural Variability}

\subsubsection{Uterine Papillary Serous Carcinoma}

Although there was decreased $\mathrm{E}$ cadherin and $\mathrm{P}$ cadherin expression at the invasive edge compared to the viable central part of the tumour, Wilcoxon Signed Ranks Test failed to reach significance (Table 5.2). All E cadherin and P cadherin expression was membranous (Figures 5.1 and 5.2 respectively).

There was significantly greater expression of $\beta$ catenin at the invasive edge compared to the viable central part of the tumour $(\mathrm{P}=0.03$, Wilcoxon Signed Ranks Test, Table 5.2). However, all $\beta$ catenin expression was membranous (Figure 5.3).

\subsubsection{Endometrioid Endometrial Carcinoma}

Although there was decreased $\mathrm{E}$ cadherin and $\mathrm{P}$ cadherin expression at the invasive edge compared to the viable central part of the tumour, Wilcoxon Signed Ranks Test failed to reach significance (Table 5.2). All E cadherin and P cadherin expression was membranous (Figures 5.5 and 5.6 respectively). There was no difference in expression of $\beta$ catenin between the viable part of the central tumour or the invasive edge. Two of the twenty EEC cases showed nuclear $\beta$ catenin expression (Figure 5.7). The remainder of the EEC cases showed membranous $\beta$ catenin staining. 
Figure 5.1

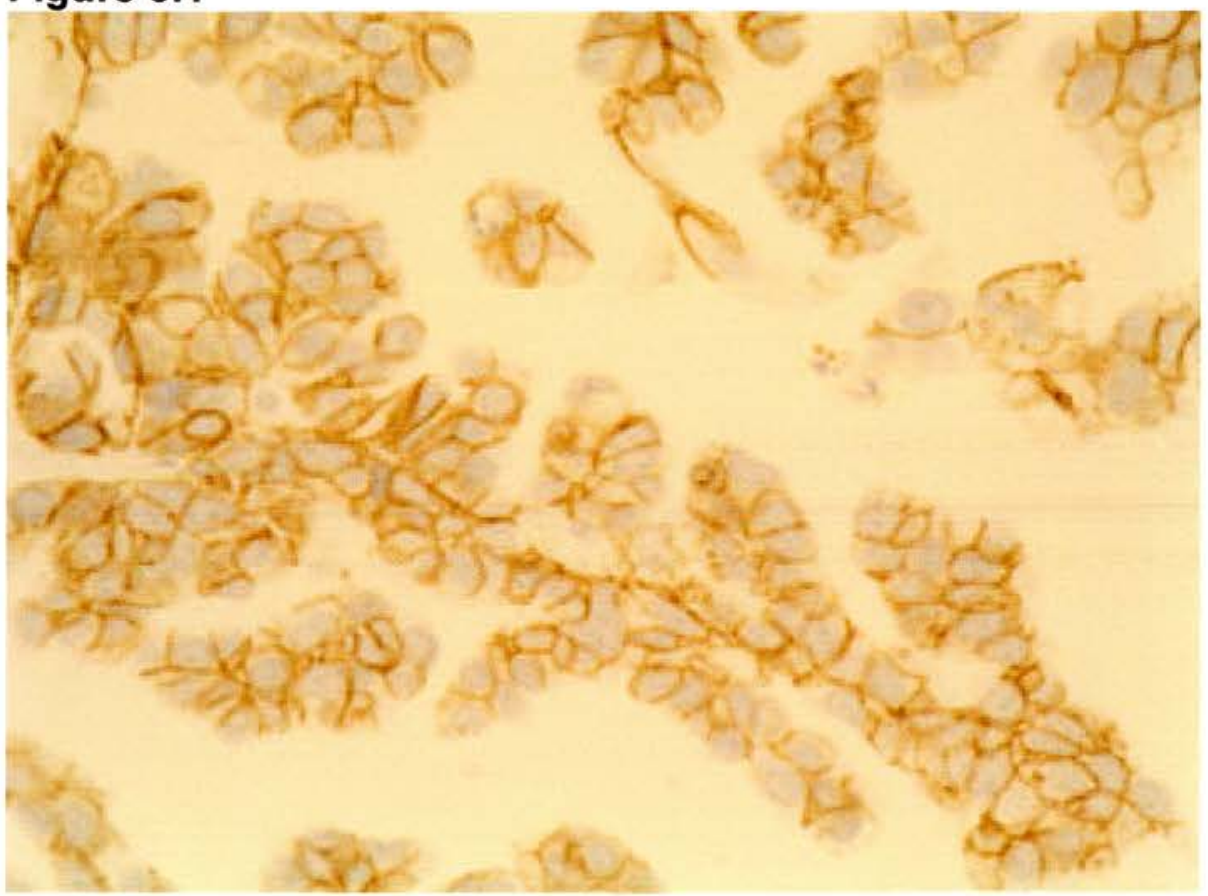

Figure 5.2

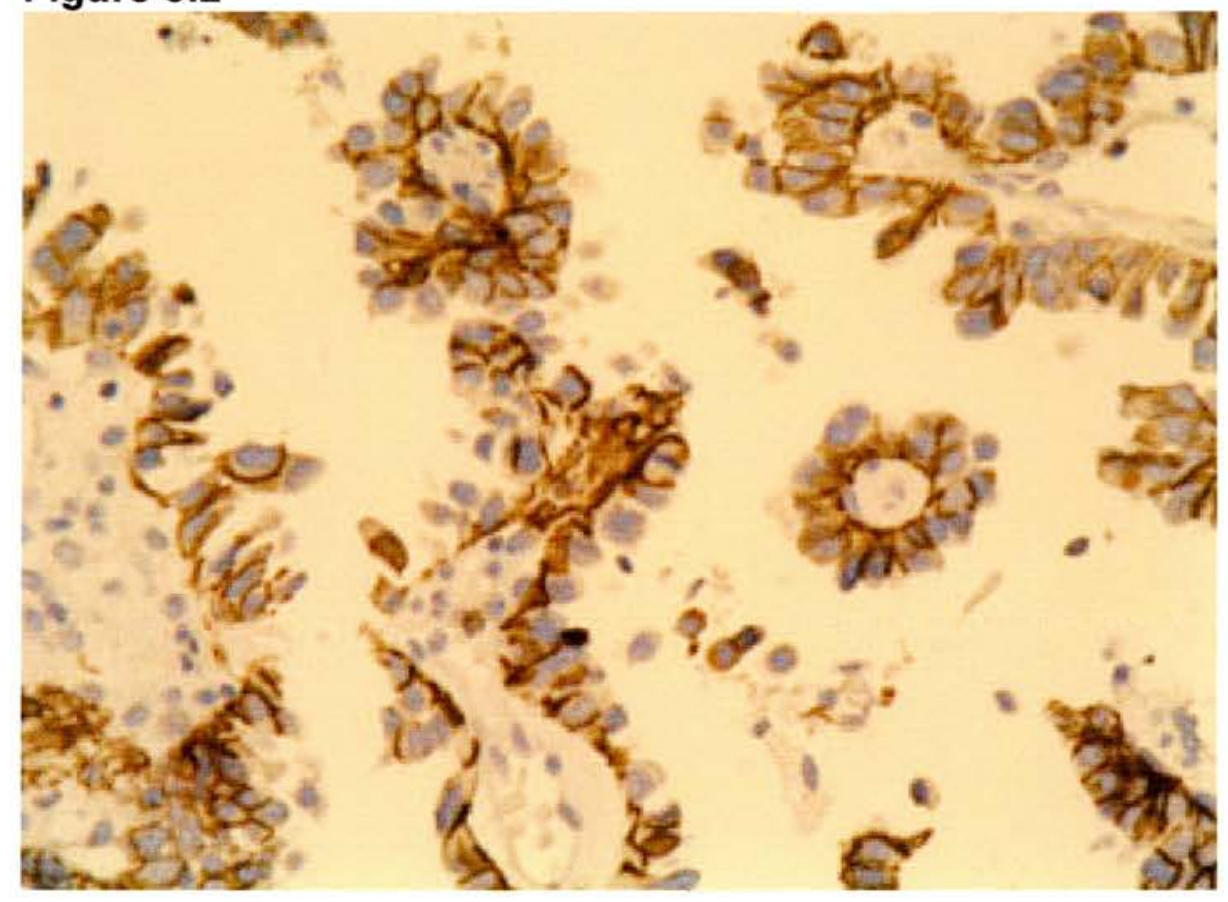

Figure 5.1. Membranous E Cadherin Expression in UPSC (Magnificationx40).

Figure 5.2. Membranous P Cadherin Expression in UPSC (Magnificationx40). 
Figure 5.3

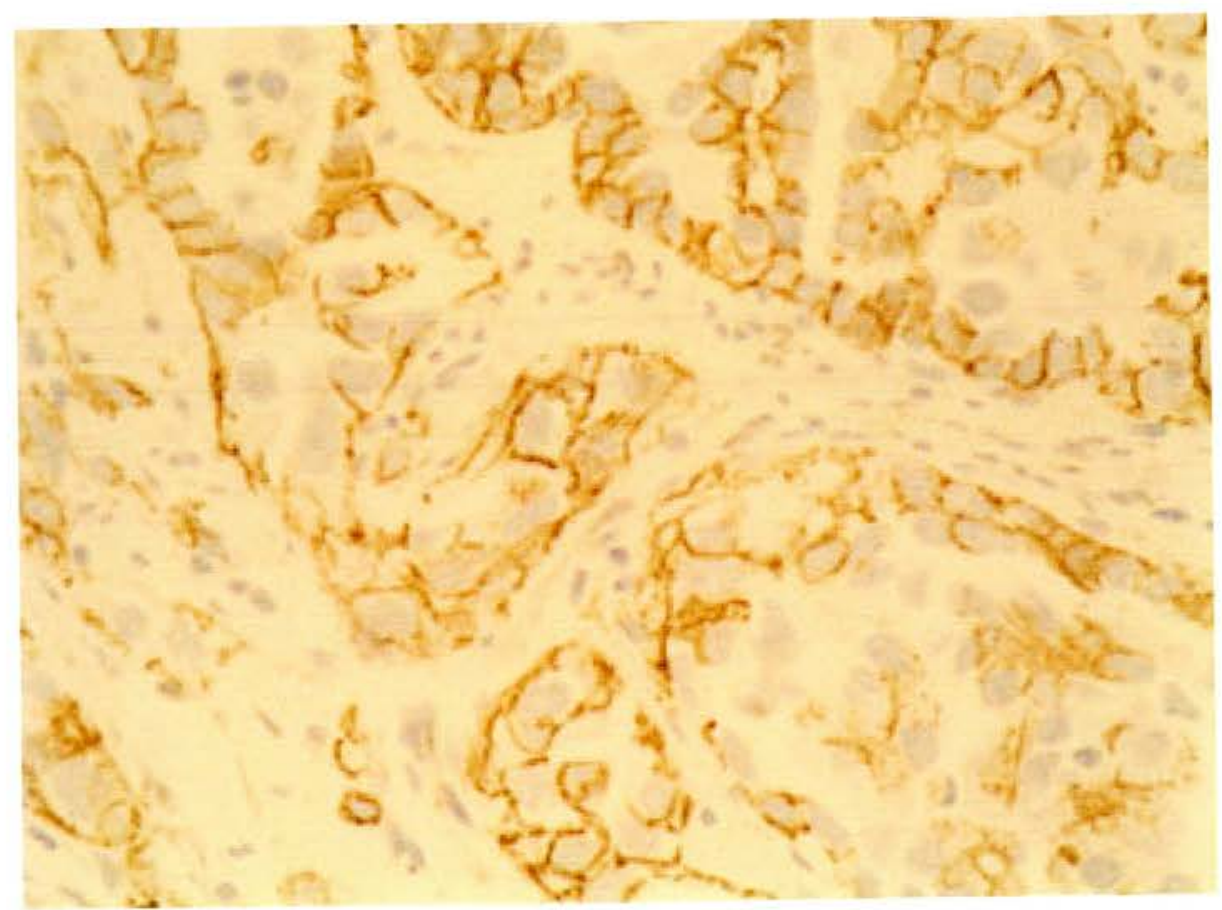

Figure 5.3. Membranous $\beta$ catenin expression in UPSC (Magnificationx40). 
Table 5.2. Expression of $E$ Cadherin, $P$ Cadherin and $\beta$ Catenin in the Central Part of the Tumour and at the Invasive Edge.

\begin{tabular}{|c|c|c|c|c|}
\hline \multirow[b]{2}{*}{ Antibody } & \multicolumn{2}{|l|}{ UPSC } & \multicolumn{2}{|l|}{ EEC } \\
\hline & Tumour & $\begin{array}{l}\text { Invasive } \\
\text { Edge }\end{array}$ & Tumour & $\begin{array}{l}\text { Invasive } \\
\text { Edge }\end{array}$ \\
\hline \multirow{2}{*}{ E Cadherin } & 7.06 & 6.96 & 7.41 & 5.46 \\
\hline & SD 1.69 & SD 2.27 & SD 2.91 & SD 3.81 \\
\hline \multirow{2}{*}{ P Cadherin } & 7.53 & 6.26 & 7.25 & 4.52 \\
\hline & SD 1.9 & SD 1.99 & SD 3.1 & SD 3.32 \\
\hline \multirow{2}{*}{$\beta$ Catenin } & ${ }^{* *} 7.04$ & **7.94 & 7.78 & 7.62 \\
\hline & SD 2.0 & SD 1.65 & SD 1.7 & SD 1.85 \\
\hline
\end{tabular}

$\mathrm{SD}=$ Standard Deviation

* $\mathrm{P}=0.08$ (Wilcoxon Signed Ranks Test)

** $\mathrm{P}=0.03$ (Wilcoxon Signed Ranks Test) 


\section{Figure 5.4}

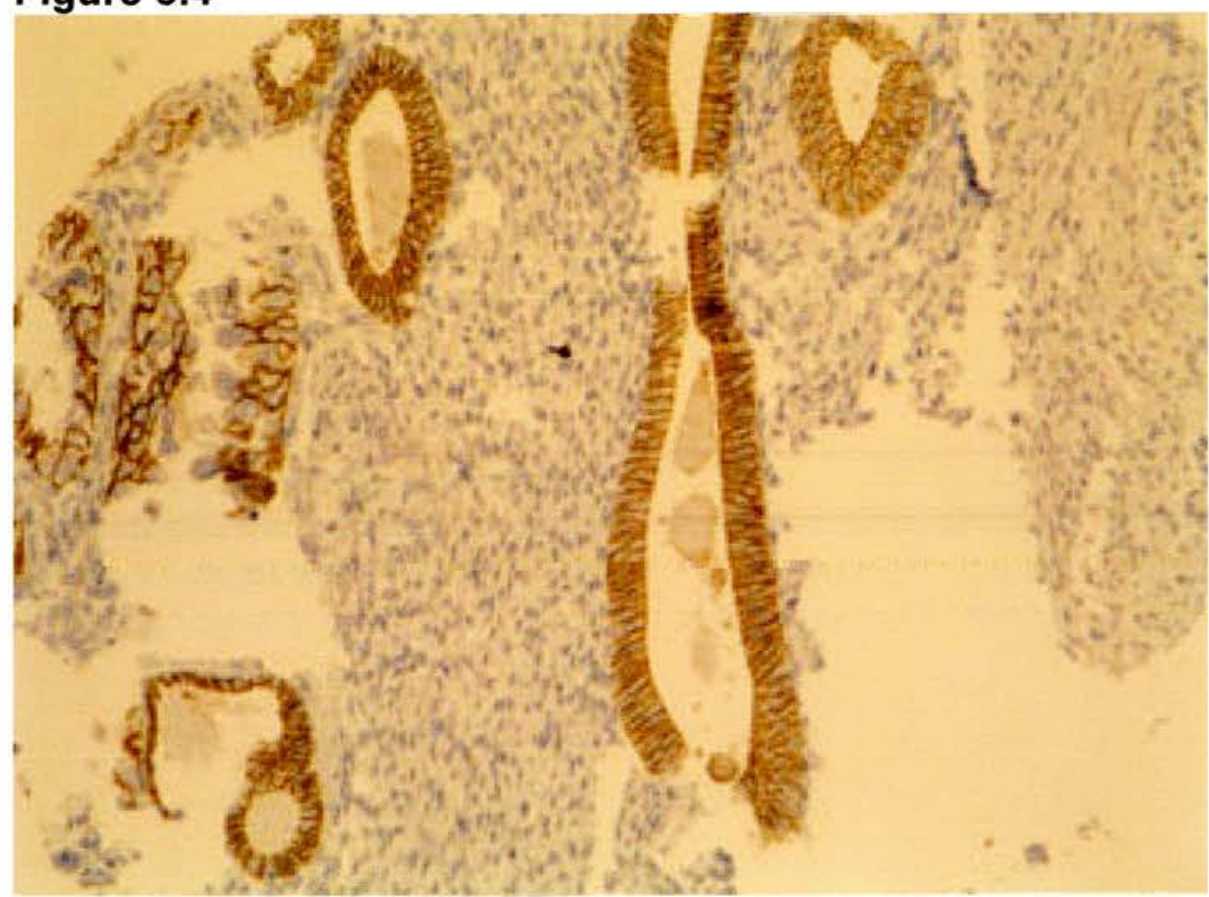

Figure 5.5

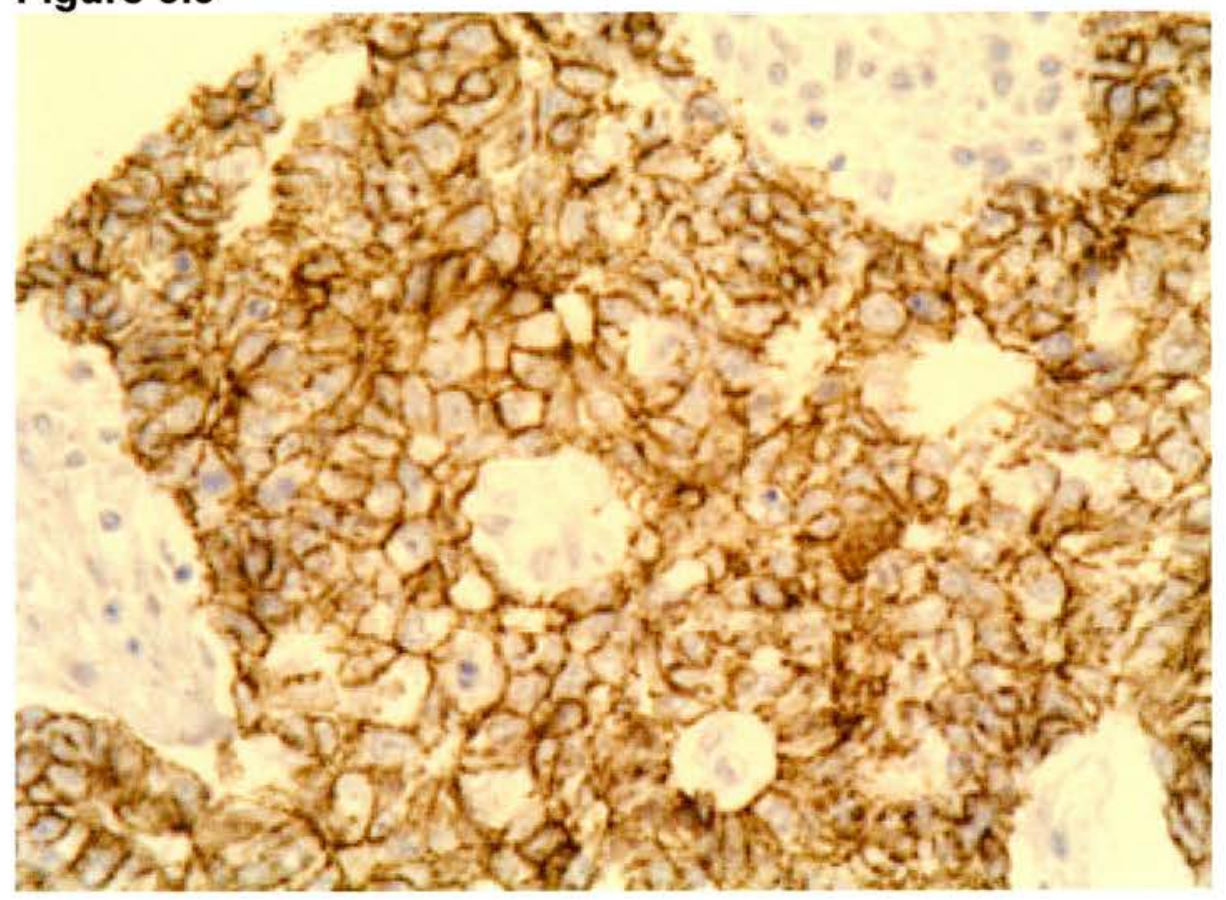

Figure 5.4. E Cadherin Expression in Normal Endometrial Epithelium (Magnificationx10).

Figure 5.5. E Cadherin Expression in EEC (Magnificationx40). 

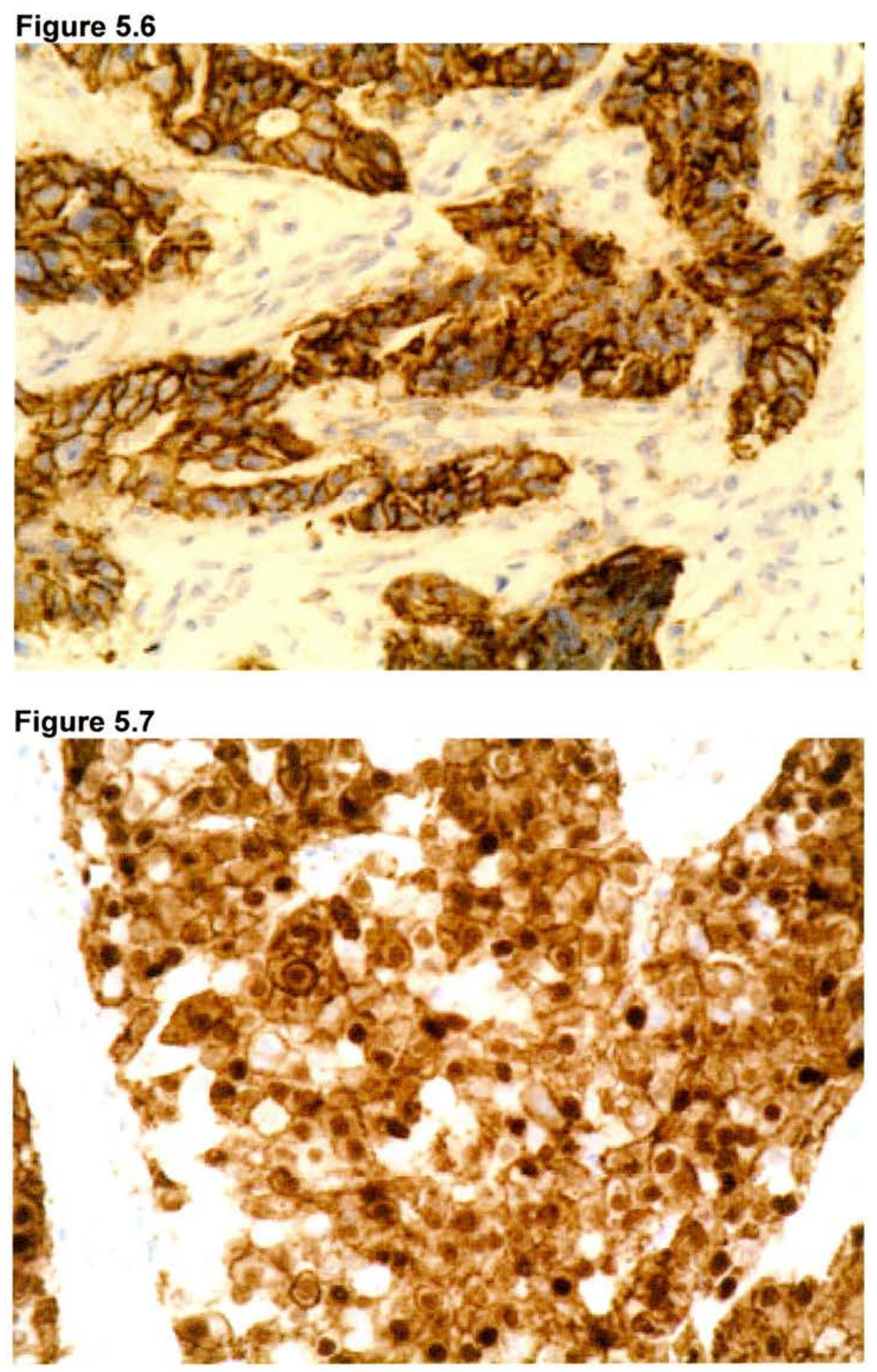

Figure 5.6. Membranous P Cadherin Expression in EEC (Magnificationx40).

Figure 5.7. Nuclear $\beta$ catenin Expression in EEC (Magnificationx40). 


\subsection{Discussion}

UPSC is an aggressive endometrial tumour with a notable propensity for lymphovascular space invasion and a poor prognosis. The cadherin-catenin complex is well described and disturbances in this are associated with a variety of cancers \{Van Roy, 1992;Birchmeier, 1993;Guilford, 1998;Perl, 1998\}. In addition, alteration in expression of both E- and P cadherin are associated with poor prognosis in many tumours \{Pijnenborg, 2004;Leblanc, 2001;Sakuragi, 1994;Holcomb, 2002\}. Along with its role in cell-cell adhesion, $\beta$ catenin also affects cell proliferation via its role in the Wnt pathway. Although the expression of E-, P- cadherin and $\beta$ catenin has been previously described in endometrial carcinoma, this chapter aimed to further investigate the differential expression between the tumour types and also within tumours, focussing on differences in expression between the viable central part of the tumour and the invasive edge.

We confirmed the findings of Shih et al, Holcomb et al and Moreno-Bueno et al \{Shih, 2004;Holcomb, 2002;Moreno-Bueno, 2003\} and demonstrated that although there was decreased $\mathrm{E}$ cadherin expression in both tumour types compared to the normal endometrium, the decrease in E cadherin expression was greater in UPSC than in EEC.

The finding of significantly lower E cadherin expression in UPSC compared to EEC may partly reflect the different biological properties of these tumours. EEC is a tumour which usually arises on a background of oestrogen excess, and it commonly expresses ER and PR and can produce intratumoural oestrogen using aromatase \{Segawa, 2005\}. On the other hand, UPSC usually arises from atrophic 
endometrium, is generally ER negative and does not produce intratumoural oestrogen. We found significantly higher ER and PR expression in our EEC cases compared to UPSC (results shown in chapter 7).

To support the theory that the variation in E cadherin expression between EEC and UPSC is due to the difference hormone environments of these tumours, Fujimoto et al \{Fujimoto, 1998\} demonstrated that the mRNA levels of $\mathrm{E}$ cadherin, $\alpha$ - and $\beta$ catenin in the endometrium in the proliferative phase were significantly less than those in the secretory phase. In addition, treatment with oestradiol dipropionate significantly reduced their levels in the secretory phase. They suggested that sex steroids could affect the adherens junction in endometrial epithelium. However a separate study showed that this effect could be reversed by treatment with progestins \{Fujimoto, 1996\} Therefore whilst oestrogen might play a part in lowering $\mathrm{E}$ cadherin in EEC, it is likely that the decrease in UPSC is due to a separate pathway. In addition PTEN is involved in the tyrosine phosphorylation of $\beta$ catenin and thus destabilisation of the cadherin catenin complex \{Vogelmann, 2005\}. It is well recognised that PTEN mutations occur in EEC but not in UPSC \{Lax, 2004\}.

Two of the EEC cases showed no E cadherin staining at all. This is an interesting finding as Pijnenborg et al \{Pijnenborg, 2004\} found that absence of E cadherin expression was predictive for distant metastases, but not local recurrence. However the ethics submission for this study limited the investigations to immunohistochemistry. Therefore follow up data for the patients could not be obtained.

In addition to confirming these established observations, the present study looked for differences in expression of these proteins between the viable central part of the 
tumour and at the invasive edge. In both tumour types we found a decrease in $\mathrm{E}$ cadherin at the invasive edge of the tumour compared with the viable central part of the tumour, although the difference did not reach significance for either UPSC or EEC. The loss of $\mathrm{E}$ cadherin at the invasive edge of these tumours might reflect decreased cell-cell adhesion at the invasive edge and thus play a part in the mechanism of invasion for both tumours.

We demonstrated P cadherin expression in both EEC and UPSC. This is in keeping with previous studies on inflammatory bowel disease and in some cancers including breast and cervical adenocarcinoma where up regulation has been demonstrated \{Sanders, 2000;Palacios, 1995;Han, 2000\}. P cadherin expression was also significantly higher in UPSC compared to EEC and this is supported by previous studies, which have shown an association with negative ER and PR status. We also noted a significant decrease in $\mathrm{P}$ cadherin expression at the invasive edge of the EEC compared with the viable central part of the tumour. Although there was a decrease in expression between the viable central part of the tumour and at the invasive edge of the UPSC cases, this did not reach significance. The decrease in P cadherin at the invasive edge of both tumours raises the possibility that its main role in the viable central part of the tumours is related to cell proliferation and dedifferentiation. However, as studies on melanoma cells have shown secretion of a truncated form of P cadherin, which disrupts cell-cell adhesion \{Bauer, 2005;Hoek, 2004\}, it is possible that the decreased expression of $\mathrm{P}$ cadherin at the invasive edge of the tumours is due to a similar process, which cannot be detected in this study as the antibody used in this study binds with the $\mathrm{C}$ terminal rather than at the $\mathrm{N}$ terminal. It 
would be interesting to further investigate this finding and to see if secretion of the truncated form also occurs in endometrial carcinoma. However, this is beyond the scope of this chapter.

$\beta$ catenin was present in both UPSC and EEC. This is in keeping with the published literature \{Shih, 2004;Moreno-Bueno, 2003;Moreno-Bueno, 2002;Palacios, 2001 \}. All UPSC cases and the majority of EEC cases demonstrated membranous expression of $\beta$ catenin and only 2 EEC cases (10\%) demonstrated nuclear expression. Our finding that $10 \%$ of EEC cases showed nuclear expression is less than that in the literature where nuclear $\beta$ catenin expression has been demonstrated in $31 \%$ of EECs and $3 \%$ of non-endometrioid endometrial carcinomas \{Nei, 1999;Moreno-Bueno, 2004;Moreno-Bueno, 2002;Palacios， 2001;Moreno-Bueno, 2003 . It is possible that this discrepancy is due to our case selection of only high grade EEC, and $\beta$ catenin mutations may be more important in the development of Grade 1 and Grade 2 EEC. In addition, those cases, which showed nuclear $\beta$ catenin expression, maintained this pattern of expression in both the viable central part of the tumour and at the invasive edge. There were no cases where the pattern of expression changed between the central part of the tumour and at the invasive edge, suggesting that $\beta$ catenin mutation is an early event in the development of endometrial carcinoma and does not influence the capacity for invasion. Nei et al $\{$ Nei, 1999\} showed that $\beta$ catenin expression was highest in those cases with nuclear $\beta$ catenin rather than the samples with membranous staining using western blotting. They postulated that the $\beta$ catenin /Wnt-1 signal transduction was highly activated in carcinogenesis of the endometrium. 
Although there was a lower level of expression of $\beta$ catenin in UPSC compared to EEC, this did not reach significance. This difference may be due to the fact that EEC is associated with ER and PR excess, whereas UPSC classically is an ER and PR negative tumour. Progesterone, but not $\beta$ oestradiol has been shown to increase $\beta$ catenin mRNA levels in cultured human endometrial stromal cells \{Chen, 1998\}, and $\beta$ catenin levels are known to be higher in the proliferative phase of the menstrual cycle rather than the secretory phase \{Fujimoto, 1998\}. Therefore it is possible that the differential secretion may be due to increased expression of PR and thus increased sensitivity to any circulating progesterone in EEC compared to UPSC.

As well as roles associated with cadherin function, $\beta$ catenin is also involved in intracellular signalling, as it is a member of the Wnt pathway, which controls cell proliferation. The activation of the Wnt signalling pathway due to $\beta$ catenin mutations has been implicated in the development of some endometrial carcinomas $\{$ Kariola, 2005\}. However, up to $25 \%$ of endometrial carcinomas have $\beta$ catenin nuclear accumulation without evidence of $\beta$ catenin mutations, suggesting alterations in other molecules that can modulate the Wnt pathway such as APC, $\gamma$ catenin and Axin $\{$ Koppert, 2004;Liu, 2000;Satoh, 2000;Jin, 2003\}.

Some studies have shown an inverse correlation between nuclear $\beta$ catenin expression and $\mathrm{E}$ cadherin expression in normal endometrium and endometrial cancers $\{$ Shih, 2004\}. However, other studies have not demonstrated this $\{$ MorenoBueno, 2003\}. As only $10 \%$ of our EEC cases and none of the UPSC cases showed 
nuclear $\beta$ catenin expression, statistical associations could not be determined. As UPSC shows a greater loss of E cadherin than EEC, but not a correspondingly higher incidence of nuclear $\beta$ catenin expression, then it is possible that the correlation demonstrated by Shih et al \{Shih, 2004\} may only be relevant to EEC. To support this, some in vitro studies have demonstrated that cell lines with inactive E cadherin as a result of CDH1 mutations do not display constitutive Wnt signalling \{van de, $2001\}$.

The membranous $\beta$ catenin expression at the invasive edge of UPSC was significantly higher than that seen in the central viable part of the tumour. This was in contrast to the pattern of expression of $\mathrm{E}$ cadherin and $\mathrm{P}$ cadherin in UPSC where both showed a non-significant decrease in expression in at the invasive edge. This pattern of expression was similar to that seen in EEC where there was no difference in $\beta$ catenin expression between the viable central part of the tumour and at the invasive edge, whereas both $\mathrm{E}$ cadherin and $\mathrm{P}$ cadherin expression was significantly lower at the invasive edge of the tumour. The decrease in $\mathrm{E}$ cadherin and $\mathrm{P}$ cadherin expression relative to $\beta$ catenin raises the possibility that the relative increase in $\beta$ catenin expression at the invasive edge of the tumours is due to activation of intracellular signalling along a separate pathway such as the Wnt signalling pathway.

\subsubsection{Summary}

This chapter has confirmed that $E$ cadherin, $P$ cadherin and $\beta$ catenin are expressed in high grade endometrial carcinoma and that there is differential $\mathrm{E}$ cadherin and $\mathrm{P}$ cadherin expression between UPSC and EEC. This is in part may be due to the important role of oestrogen and progesterone in the development and progression of 
EEC compared to UPSC. In addition we have shown alteration of expression of E cadherin, $\mathrm{P}$ cadherin and $\beta$ catenin between the viable central part of the tumour and the invasive edge, supporting the theory of the invasive subclone acquiring mutations in order to invade and metastasise. 


\section{Chapter 6}

\section{The Expression of CD98 and Galectin-3 in Uterine Papillary Serous Carcinoma and Endometrioid Endometrial Carcinoma}

\subsection{Introduction}

Endometrioid endometrial carcinoma (EEC) is the most commonly diagnosed type of endometrial carcinoma, comprising $80 \%$ of all cases. Uterine papillary serous carcinoma (UPSC) accounts for only $10 \%$ of cases \{Burton, 1998\}, but it accounts for a much smaller proportion of Stage I endometrial carcinomas \{Hendrickson, 1983;Bancher-Todesca, 1998;Dembo, 1985 . UPSC is an aggressive tumour, with a propensity for lymphovascular space invasion and peritoneal metastases.

Invasion and metastasis of tumour cells requires a variety of complex interactions including cell-cell interactions, cell-matrix interactions and degradation of the extra cellular matrix (ECM). For metastasis to occur, cancer cells must detach from each other, attach to matrix components, degrade the ECM and then migrate into the blood stream which involves binding to the endothelium in target organ microvessels $\{\mathrm{Al}$ Mehdi, 2000;Chambers, 2002\}. This chapter focuses on the attachment of tumour cells to matrix components. Tumour cells adhere not only to themselves but also to the ECM and endothelial cells as part of the process of invasion and metastasis and tumour cell adhesion is mediated by specific interactions between cell surface lectins and their carbohydrate ligands presented on glycoproteins and glycolipids \{Glinsky, 2003;Orr, 2000;Inohara, 1996;Kannagi, 1997;Inohara, 1995\}. 


\subsubsection{CD98.}

CD98 is a disulphide-linked $125 \mathrm{kDa}$ heterodimeric membrane glycoprotein composed of a glycosylated $85 \mathrm{kDa}$ heavy chain and one of at least six alternative non-glycosylated $40 \mathrm{kDa}$ light chains. CD98 is encoded on chromosome 11q13 \{Haynes, 1981;Hemler, 1982\} and it is found on the cell surface of most normal cells and some tumour cells and has been shown to be involved in various cellular activities including cellular proliferation, cell transformation, cell fusion and cell adhesion \{Yagita, 1986;Haynes, 1981;Hemler, 1982;Hara, 1999;Fenczik, 1997\}.

CD98 was originally identified as a cell surface antigen associated with lymphocyte activation \{Haynes, 1981;Hemler, 1982\}. Further experiments have shown that CD98 is quickly induced from low levels in quiescent cells to higher levels, following cellular activation. It is upregulated early in transition from G0 to G1 phase of the cell cycle and remains at elevated levels until the cell cycle is complete.

CD98 has been identified as a unique and highly specific regulator of integrin affinity \{Fenczik, 2001\}. It has been shown to stimulate adhesion of breast cancer cells to laminin via $\alpha 3 \beta 1$ integrin $\{$ Chandrasekaran, 1999$\}$ and recent studies have shown CD98 to co-localise with $\beta 1$ integrin on the cell membrane of keratinocytes and to promote integrin-like signalling \{Lemaitre, 2005; Rintoul, 2002\}. It is possible that by cross-linking $\mathrm{CD} 98, \mathrm{CD} 98$ acts as a facilitator in the plasma membrane, clustering $\beta 1$ integrins to form high density complexes, thus subsequently leading to integrin activation and adhesion, integrin signalling and anchorage independent growth. In addition, it has been shown that $\beta 1$ integrinmediated adhesion of the small cell lung cancer cell line H345 to fibronectin and 
laminin can be markedly upregulated by cross-linking CD98 \{Fenczik, 1997\}. Kakugawa et al \{Kakugawa, 2003\} also demonstrated that CD98 stimulation could activate CEA-CAM-1 mediated cell adhesion independently of integrins.

The heavy chain of CD98 is an integral membrane protein with a single membrane spanning domain, classified as a type II membrane glycoprotein \{Teixeira, 1987; Quackenbush, 1987;Lumadue, 1987\}. Hara et al \{Hara, 2000\} demonstrated that truncation of the extracellular domain of the CD98 heavy chain enhanced tumorigenicity in cell lines. Both the extracellular domain and the cytoplasmic tail of CD98 appear to be crucial for its action and CD98 that lacks either a cytoplasmic domain or the unpaired cysteine in the extracellular domain disrupts virus-induced cell fusion and does not inhibit Tac- $\beta 1$ suppression of integrin affinity.

Six CD98 light chains have been identified, all of which are associated with L-type amino acid transport (LAT) activity \{Mannion, 1998;Kanai, 1998; Torrents, 1998;Estevez, 1998;Mastroberardino, 1998;Pfeiffer, 1998;Pfeiffer, 1999;Tsurudome, 1999 \}. LAT-1 protein is widely distributed on normal cells and is involved with cellular amino acid uptake. The LAT-2 protein is predominantly expressed in renal proximal tubules and the CD98 heavy chain has been shown to control delivery of the LAT-2 protein to the membrane in a Xenopus oocyte model system \{Kanai, 1998;Mastroberardino, 1998;Mannion, 1998;Nakamura, 1999;Pineda, 1999;Segawa, 1999;Torrents, 1998;Pfeiffer, 1999;Kanai, 2000;Sato, 1999;Fukasawa, 2000;Pineda, 1999; Rossier, 1999\}. In transformed cells it is thought that L-type amino acid 
transporters are upregulated to support the high level protein synthesis for continuous growth and proliferation \{Christensen, 1990\}.

The pattern of expression of CD98 varies between tumours. Studies have shown that the CD98 heavy chain is overexpressed in oral squamous cell carcinoma and in bladder carcinomas $\{$ Kim, 2002;Kim, 2004\}. Esteban et al \{Esteban, 1990\} found that CD98 was present on all squamous cell carcinomas of the larynx and that a diffuse pattern of expression was associated with more extensive disease and poorer differentiation. In contrast, other studies have shown down-regulation of CD98 gene expression in cell lines made from metastatic adenoid cystic carcinoma when compared to cell lines created from adenoid cystic carcinomas that had not metastasised \{Huang, 2003\}. To date, no studies have been published on the pattern of expression of CD98 in endometrial carcinomas. However, previous studies examining in vivo integrin expression in normal endometrium showed that $\beta 1$ integrin expression was mostly seen in endometrial stromal cells \{Castelbaum, $1997\}$. In addition, in vitro studies showed that progesterone treatment of oestradiolprimed cells resulted in increased expression of the $\alpha 1 \beta 1$ collagen-laminin receptor and suppression of the $\alpha v \beta 3$ vitronectin receptor \{Castelbaum, 1997\}. Lessey et al \{Lessey, 1995\} found alteration of integrin expression between benign and malignant endometrial epithelium, with the $\alpha 5 \beta 1$ integrin, most commonly seen on benign endometrial stromal cells, being found in almost $20 \%$ of cases of endometrial carcinoma. They also showed that integrin expression correlated with steroid receptor status, as well as with grade, stage and depth of invasion \{Lessey, 1995\}. 
Thus, mounting evidence suggests that CD98 may be important in cancer and inflammation through its effects on cellular activation and integrin-mediated adhesion.

\subsubsection{Galectin}

The galectin family of carbohydrate binding proteins currently comprises 14 members identified by their conserved sequence elements. They all have a carbohydrate binding domain, a repetitive collagen-like sequence and a functionally distinct amino-terminal domain \{Hughes, 1994;Barondes, 1994\}. They appear to show some tissue and developmental specificity where galectin-1 (Gal-1) is predominantly found in tissues derived from mesoderm, galectin-3 (Gal-3) is commonly found in epithelium and macrophages, as well as cartilage, galectin-4 (Gal-4) is identified in the gastrointestinal epithelium and galectin-7 (Gal-7) is found in stratified epithelium such as the epidermis. Galectins exist as monomers but can undergo homophilic binding to form polymers through interactions of either the $\mathrm{N}$ terminal domain or the C terminal domain \{Yang, 1998;Birdsall, 2001\}. They are synthesized on cytoplasmic ribosomes and following synthesis there is selective intraccllular targeting of specific galectins to subcompartments of the cytosol, to distinct subcellular organelles, and to the cell membrane and membrane bound vesicles.

\subsubsection{Galectin-3}

Gal-3 exists in a non-phosphorylated and a phosphorylated form. The former is found exclusively in the nucleus, while the latter is found in both the nucleus and in 
the cytoplasm suggesting that phosphorylation may be important for nuclear export of the protein \{Openo, 2000; Cherayil, 1989\}.

Possible ligands for intracellular Gal-3 include, Bcl-2, ALIX/AIP-1, Gemin4 and CBP70. Nuclear Gal-3 is thought to play a role in pre-mRNA splicing, which in turn may be related to regulation of cell growth and proliferation \{Dagher, 1995\}. Gal-3 may also act to increase metastatic potential of tumour cells by promoting tumour cell adhesion \{Raz, 1987; Inohara, 1995\}, invasiveness \{Le Marer, 1996\} and inducing endothelial cell proliferation and angiogenesis \{Nangia-Makker, 2000\}.

A further possible role for Gal-3 is in antagonising tumour cell apoptosis via Fas ligand binding and this is thought to only occur when Gal-3 is in the phosphorylated state. The exact mechanism underlying the anti-apoptotic activity of Gal-3 is unclear, but it does share a NWGR quartet in the $\mathrm{C}$ terminal part with $\mathrm{Bcl}-2$ and this has been shown to be critical for the anti apoptotic function Bcl-2. However it is not known whether these proteins interact in vivo, as Gal-3 expression does not alter the expression levels of Bcl-2 family members including Bcl-xL and Bax Akahani, 1997; Matarrese, 2000;Yu, 2002\}.

Gal-3 expression varies between tumour types. It is highly expressed in head and neck squamous cell carcinoma, melanoma, angiosarcoma and thyroid carcinoma \{Gillenwater, 1996;Raz, 1981\} In vivo expression correlates with tumour cell transformation and metastatic phenotype and it has been shown to significantly enhance tumour cell adhesion to common ECM proteins \{Raz, 1990;Ochieng, $1999\}$, increase the incidence of lung metastases $\{$ Raz, 1990\} and protect cancer cells from apoptosis \{Akahani, 1997;Matarrese, 2000;Yu, 2002\}. In addition, pre- 
treatment of tumour cells with anti-Gal-3 antibodies reduce the incidence of metastatic lung colonies by $90 \%$ \{Meromsky, 1986\}.

In contrast down regulation of Gal-3 has been demonstrated in small cell lung cancer, endometrial carcinoma and breast carcinoma \{Buttery, 2004; van den Brule, 1996;Castronovo, 1996\} and varying results have been found in colonic carcinomas \{Lotz, 1993;Bresalier, 1998\}. Squamous cell carcinoma and melanoma are not chemosensitive tumours whereas small cell lung cancer and breast carcinoma are characteristically chemosensitive at presentation, but develop resistance to chemotherapy following treatment. This suggests that up-regulation of Gal-3 confers a relative resistance to chemotherapy whereas down-regulation of Gal-3 may be associated with chemosensitivity.

Gal-3 has been identified in the nucleus, cytoplasm and at the cell membrane \{Openo, 2000; Cherayil, 1989\}.

In addition to its intracellular roles, Gal-3 is actively secreted into the extracellular tissues where potential binding proteins include CD98, laminin and fibronectin \{Dong, 1997;Sato, 1994\}.

Loss of nuclear staining of Gal-3 has been demonstrated with progression of disease in colonic carcinoma and squamous cell carcinoma of the tongue \{Lotz, 1993;Honjo, $2000\}$. However, these studies had conflicting results in that the former demonstrated an overall down-regulation of Gal-3 expression whereas the latter found an associated up-regulation of Gal +3 expression.

Van Den Brule \{van den Brule, 1996\} found up-regulation of Gal-1 and downregulation of Gal-3 in EEC in comparison to normal endometrium. In addition they 
found those cases with only cytoplasmic expression (i.e. no nuclear staining) of Gal3 had increased myometrial invasion compared to those cases with both cytoplasmic and nuclear expression of Gal-3. To date, no studies on the expression of Gal-3 in any other types of endometrial carcinoma have been performed.

\subsubsection{Summary}

This chapter attempted to determine if differences in interaction between tumour cells and the extracellular matrix were responsible for the recognised differences in clinical behaviour between UPSC and EEC. As no studies of CD98 expression in endometrial carcinomas have been published and CD98 expression varies according to tumour type in other carcinomas, this chapter aimed to determine the pattern of expression of CD98 in EEC and UPSC. As down regulation of CD98 has been found in metastatic adenoid cystic carcinoma, this chapter also focussed on differential expression of CD98 between the central viable part of the tumour and the invasive edge. Gal-3 is a recognised ligand of CD98, so it was also included in this chapter. The expression of Gal-3 has been studied in EEC, but not in UPSC. Therefore this chapter was undertaken to determine whether there is differential expression of CD98 and Gal-3 between EEC and UPSC, which might explain the increased aggressiveness of UPSC compared to EEC. 


\subsection{Materials and Methods}

\subsubsection{Tissues}

Tissues were obtained as described in "Materials and Methods", Section 2.1.

\subsubsection{Tissue Microarray}

The tissue microarray was constructed as described in "Materials and Methods", Section 2.2 .

\subsubsection{Immunohistochemistry}

Immunohistochemistry was performed for CD98 and Gal-3 using the technique described in "Materials and Methods", Sections 2.3.7 and 2.3.8 respectively.

\subsubsection{Scoring}

Scoring of antibody expression was assessed using the technique as described in "Materials and Methods", Section 2.4.

\subsubsection{Statistical Analysis}

Statistical analysis was performed as described in "Materials and Methods", Section 2.5 . 


\subsection{Results}

\subsubsection{Intertumoural Variability}

There was significantly greater CD98 expression in EEC compared to UPSC (P = 0.009, Mann Whitney U Test, Table 6.1). All CD98 expression was membranous. Figures 6.1 and 6.2 show the pattern of expression of CD98 in EEC and UPSC respectively.

Although Gal-3 expression was higher in UPSC compared to EEC, this did not reach significance. All Gal- 3 expression was either cytoplasmic and nuclear, or cytoplasmic only. There was no difference in the pattern of expression between UPSC and EEC; Figures 6.3 and 6.4 show the pattern of expression of Gal-3 in EEC and UPSC respectively. Stromal Gal-3 staining was identified in very few cases of both UPSC and EEC. The number of cases involved was too small for statistical analysis.

Table 6.1. Expression of Galectin-3 and CD98 in the Viable Central Part of the Tumour in UPSC and EEC.

\begin{tabular}{|l|l|l|}
\hline Antibody & UPSC & EEC \\
\hline CD98 & $\begin{array}{l}* 4.32 \\
\text { SD 2.48 }\end{array}$ & $\begin{array}{c}\text { S.17 } 2.53 \\
\text { Galectin 3 }\end{array}$ \\
& 4.75 & 3.82 \\
& SD 2.18 & SD 2.30 \\
\hline
\end{tabular}

$\mathrm{SD}=$ Standard Deviation

$* \mathrm{P}=0.009$ (Mann Whitney U Test) 


\section{Figure 6.1}

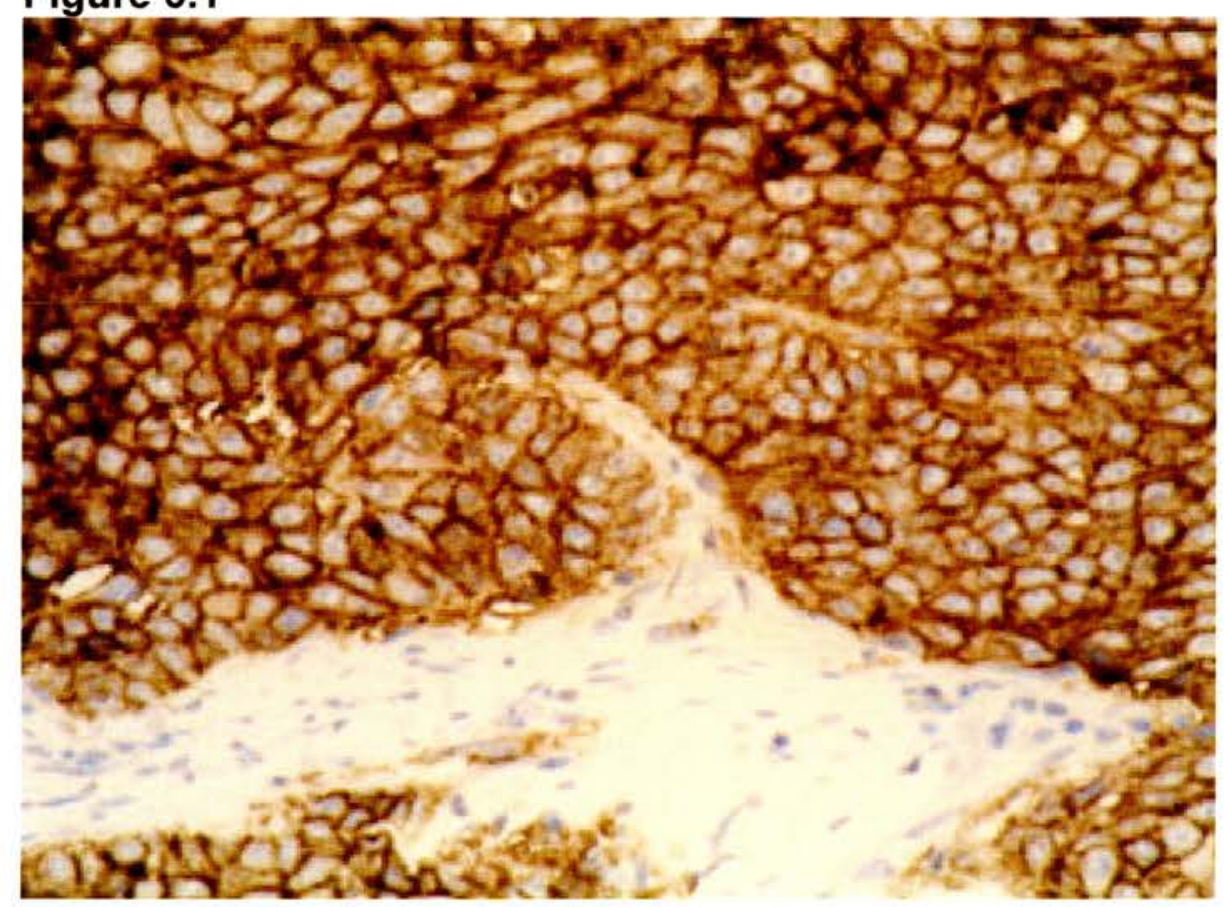

Figure 6.2

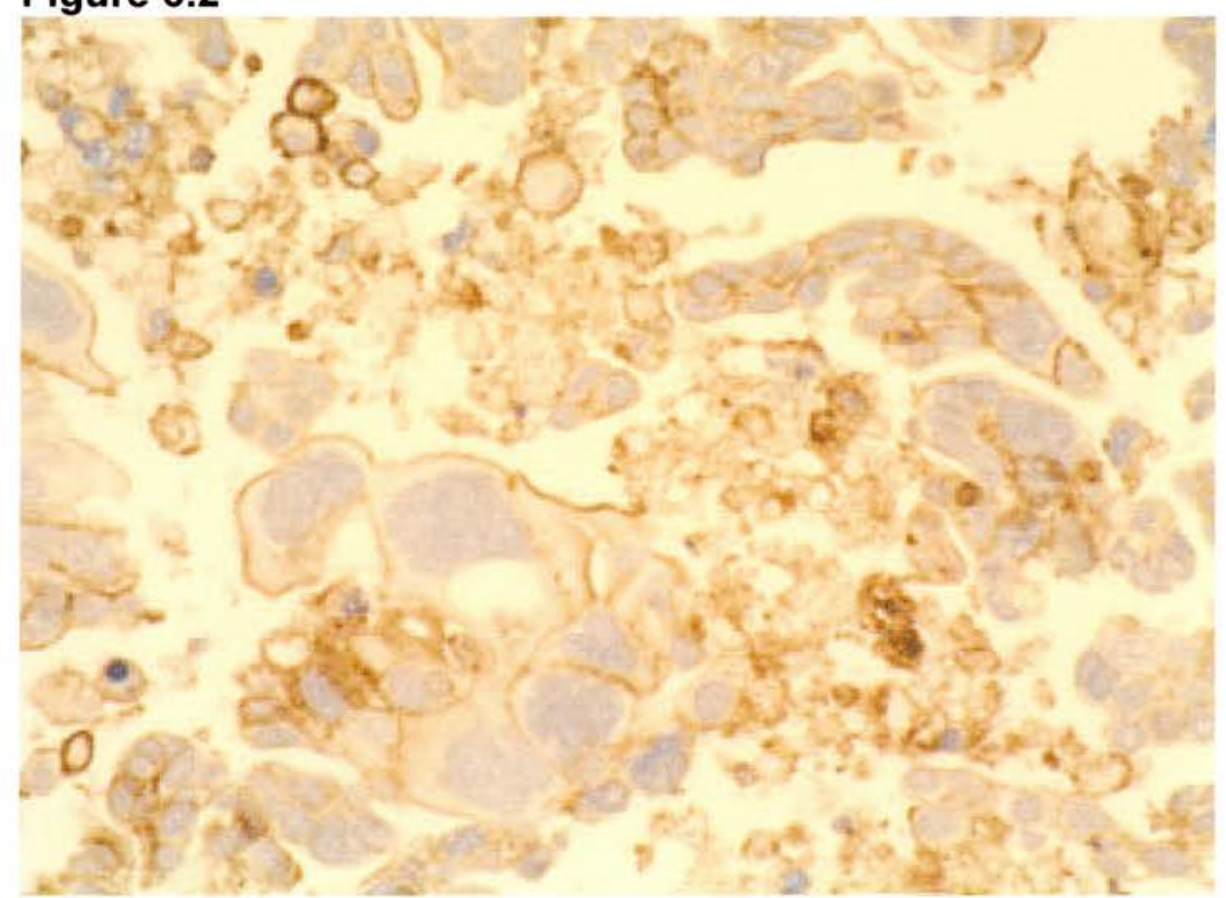

Figure 6.1. Membranous Expression of CD98 in EEC (Magnificationx40).

Figure 6.2. Membranous Expression of CD98 in UPSC (Magnificationx40). 


\section{Figure 6.3}

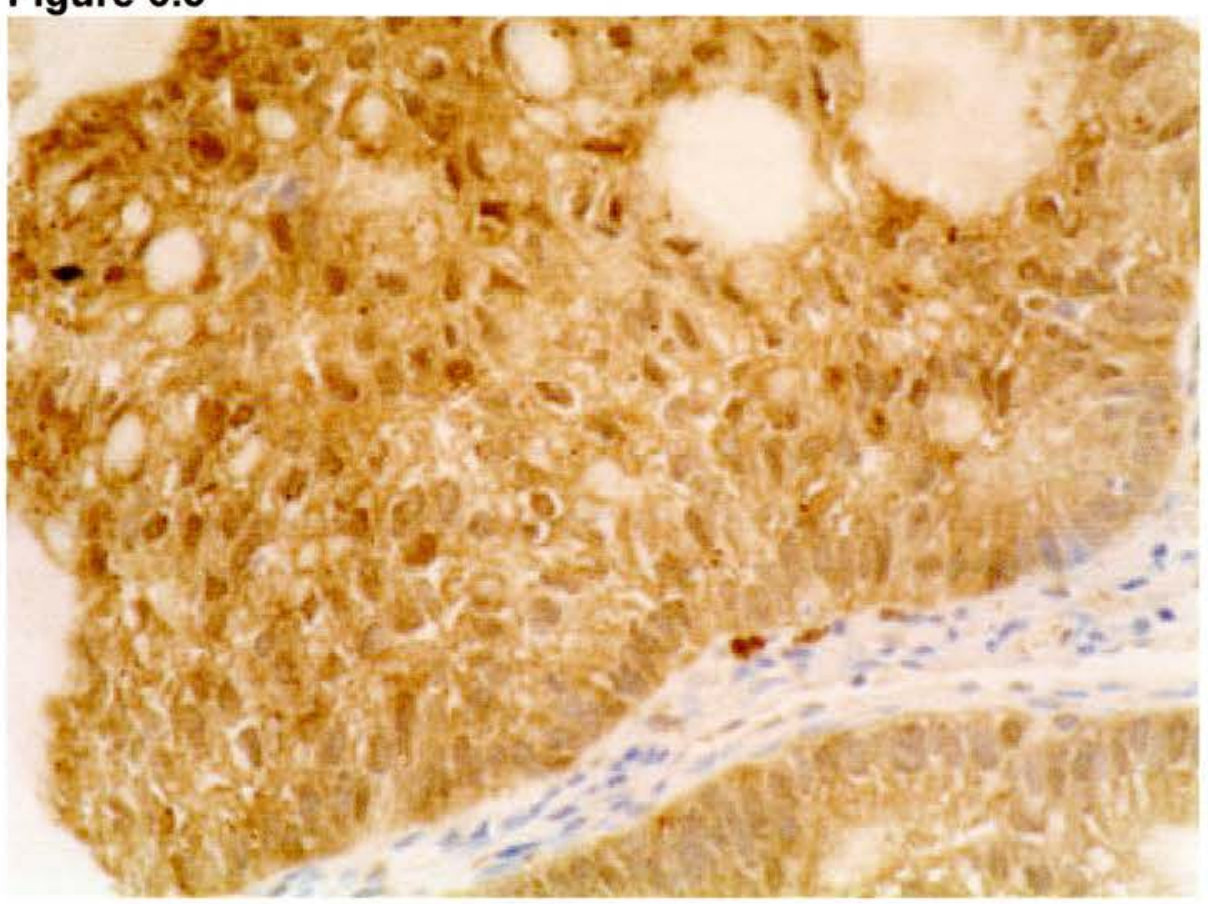

Figure 6.4

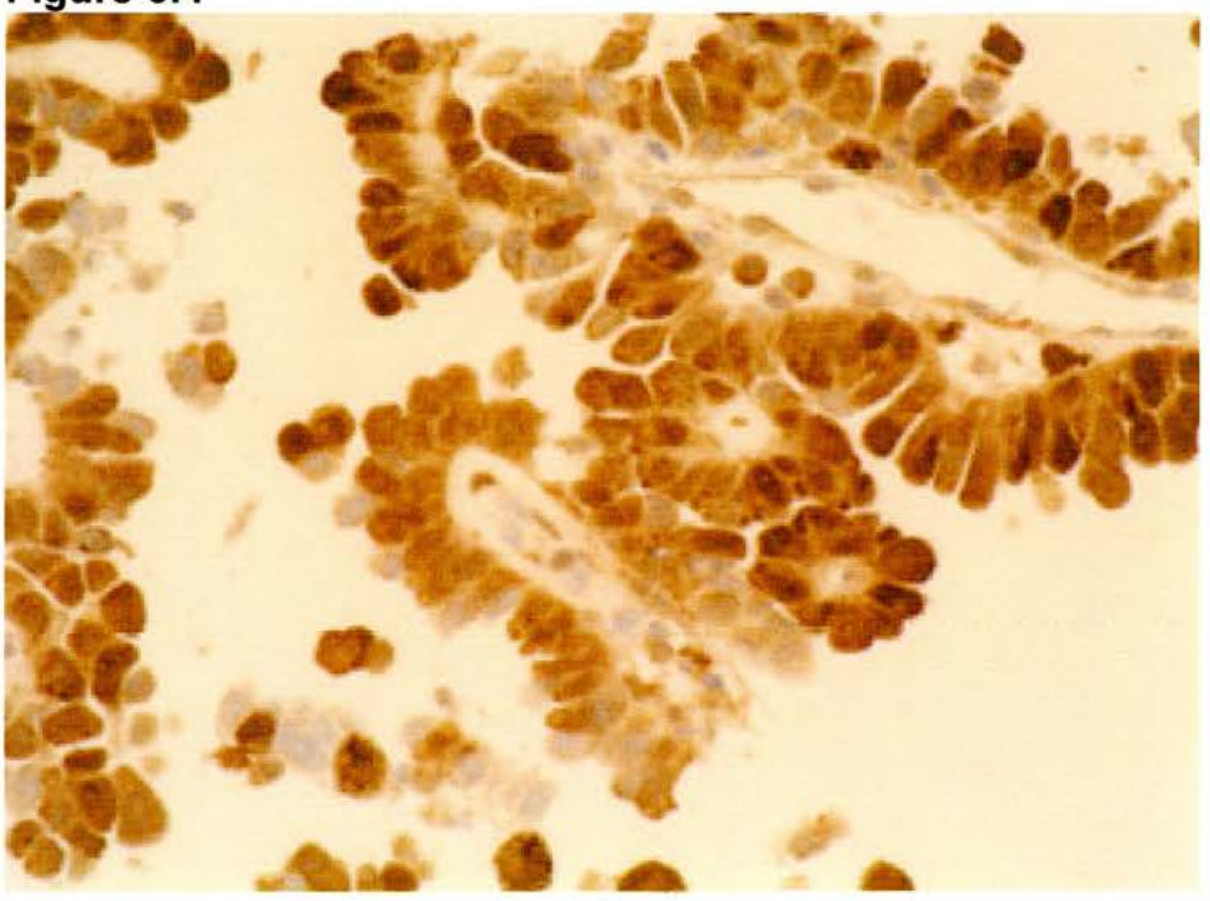

Figure 6.3. Cytoplasmic Expression of Galectin-3 in EEC (Magnificationx40).

Figure 6.4. Cytoplasmic Expression of Galectin-3 in UPSC (Magnificationx40). 


\subsubsection{Intratumoural Variability}

\subsubsection{Uterine Papillary Serous Carcinoma}

There was significantly greater expression of CD98 at the invasive edge compared to the viable central part of the tumour $(\mathrm{p}=0.005$ Wilcoxon Signed Ranks Test, Table 6.2). There was significantly less Gal-3 expression in the tumour at the invasive edge compared to the viable central part of the tumour $(\mathrm{p}=0.031$, Wilcoxon Signed Ranks Test). Although there was a decrease in the percentage of cells showing nuclear expression of Gal-3 from the viable central part of the tumour to the invasive edge, this did not reach significance (Table 6.2).

\subsubsection{Endometrioid Endometrial Carcinoma}

No significant differences in expression of Gal-3 and CD98 were noted between the invasive edge of the tumour and the viable central part of the tumour (Table 6.2). Although there was a decrease in the percentage of cells showing nuclear expression of Gal-3 from the viable central part of the tumour to the invasive edge, from $52 \%$ to $14 \%$, this did not reach significance (Table 6.2). 
Table 6.2. Expression of Galectin-3 and CD98 in the Central Part of the Tumour and at the Invasive Edge.

\begin{tabular}{|l|l|l|l|l|}
\hline \multirow{2}{*}{ Antibody } & \multicolumn{2}{|l|}{ UPSC } & \multicolumn{2}{l|}{ EEC } \\
\cline { 2 - 5 } & Tumour & $\begin{array}{l}\text { Invasive } \\
\text { Edge }\end{array}$ & Tumour & $\begin{array}{l}\text { Invasive } \\
\text { Edge }\end{array}$ \\
\hline CD98 & $\begin{array}{l}5.32^{+} \\
\text {SD } 2.48\end{array}$ & $\begin{array}{l}5.10^{+} \\
\text {SD } 1.87\end{array}$ & SD 2.53 & SD 2.01 \\
\hline Galectin 3 & $4.75^{*}$ & $3.43^{*}$ & 3.77 & 2.57 \\
Site of Gal-3 & SD 2.18 & SD 1.68 & SD 2.30 & SD 1.96 \\
Staining (\%) & & & & \\
C & 51 & 67 & 48 & 86 \\
C\&N & 49 & 33 & 52 & 14 \\
\hline
\end{tabular}

$\mathrm{C}=$ cytoplasmic staining, $\mathrm{C} \& \mathrm{~N}=$ cytoplasmic and nuclear staining .

${ }^{+} \mathrm{P}=0.005$ (Wilcoxon Signed Ranks)

${ }^{*} \mathrm{P}=0.031$ (Wilcoxon Signed Ranks) 


\subsection{Discussion}

This chapter examined the immunohistochemical pattern of expression of CD98 and Gal-3 in EEC and UPSC, with the aim of determining if differential expression may, in part, contribute to the differing clinical behaviour of these tumours.

CD98 was demonstrated in all tumours examined. The finding of CD98 in both EEC and UPSC was unsurprising as CD98 plays a role at the G1/S interphase of the cell cycle in all proliferating cells.

In addition, there was significantly higher expression of CD98 in EEC compared to UPSC. The variation in expression of CD98 may be related to its association with $\beta 1$ integrin. Studies have shown integrin expression to vary between benign and malignant endometrial epithelium. $\beta 1$ integrin is most commonly seen on benign endometrial stromal cells, but has been found in almost $20 \%$ of cases of endometrial carcinoma \{Lessey, 1995\}. In addition, $\beta 1$ integrin expression may be modulated by oestrogen and progesterone and Lessey et al \{Lessey, 1995\} found that integrin expression correlated with steroid receptor status, as well as with grade, stage and depth of invasion. Therefore if high oestrogen levels affect $\beta 1$ integrin, it is possible that CD98 expression may also be upregulated by high intratumoural oestrogen.

EEC usually arises on a background of oestrogen excess, commonly expresses oestrogen receptors (ER), and can produce intratumoural oestrogen using aromatase. In contrast, UPSC usually arises from atrophic endometrium, is generally ER negative and does not produce intratumoural oestrogen. We found significantly higher ER and progesterone receptors (PR) positivity in our EEC cases compared to UPSC (results shown in chapter 7). 
Alternatively CD98 expression may be due to activity of the LAT amino acid transporter system, making amino acids available for protein synthesis by proliferating tumour cells.

Significantly lower levels of CD98 were seen in the viable central part of UPSC when compared to the invasive edge, suggesting a different role of CD98 in UPSC compared to EEC. The increased expression of CD98 at the invasive edge of UPSC compared to the viable central part may reflect activation of the $\beta 1$ integrin signalling pathway, increased anchorage independent growth and thus invasion. It is well known that integrin is a key factor for cell invasion and migration, allowing the cancer cell to penetrate adjacent tissues and attach itself to the target tissue's basal matrix by binding to components of the extracellular matrix \{Hood, 2002\}.

In contrast, our finding of increased expression of CD98 in the viable central part of EEC and a non significant decrease at the invasive edge has similarities to the findings of Huang et al \{Huang, 2003\} who compared expression of CD98 in both primary and metastatic adenoid cystic carcinoma. It is possible that the decrease in CD98 at the invasive edge of EEC compared to the central viable part and the metastatic adenoid cystic carcinoma compared to the primary tumour reflects a similar mechanism. The loss of CD98 might cause or enable loss of tumour cell-cell adhesion in a mechanism different to that where increased expression stimulates $\beta 1$ integrin signalling and thus anchorage independent growth. The high CD98 in the viable central part of EEC may be due in part to the relative increase in PR and ER, affecting $\beta 1$ integrin status and activity and therefore CD98 indirectly, but it is also 
possible that CD98 plays a role in increasing nutrient uptake in proliferating cells via the LAT system.

Similarly to CD98, we identified Gal-3 expression in all cases examined. Although there was higher Gal-3 expression in UPSC compared to EEC, this difference did not reach significance. Gal-3 expression was significantly less at the invasive edge of UPSC and there was also a non-significant decrease in Gal-3 expression at the invasive edge of EEC when compared to viable central part of the tumour. This decrease in Gal-3 expression was also mirrored by the loss of nuclear expression of Gal-3 in a high proportion of both tumours at the invasive edge. Gal-3 expression was both nuclear and cytoplasmic and cytoplasmic alone. In the central part of both tumours the site of Gal-3 expression was equally split between nuclear and cytoplasmic and cytoplasmic alone, raising the possibility that Gal-3 was acting in a variety of roles including cell growth, proliferation and cell adhesion. At the invasive edge of both tumours a higher proportion of cases showed cytoplasmic expression only. However, this did not achieve significance. This non-significant loss of nuclear staining at the invasive edge is in keeping with previous studies showing an association with loss of nuclear expression and progression of disease \{Lotz, 1993; Castronovo, 1996;Bresalier, 1998\}. The authors of these studies suggested that nuclear expression of Gal-3 conferred specific antiapoptotic properties to the cell, whereas cytoplasmic expression conferred invasive properties to the cell.

In addition we found a significant decrease of Gal-3 expression at the invasive edge of UPSC and a non-significant decrease in Gal-3 expression at the invasive edge of EEC. This is similar to the findings of Lotz et al \{Lotz, 1993\} who showed an 
overall down regulation of Gal-3 in colonic carcinoma along with loss of nuclear expression. Lotz et al \{Lotz, 1993\} suggested that nuclear localisation of Gal-3 was associated with cessation of cell division and differentiation and therefore loss of nuclear expression may be associated with increasing cell division and dedifferentiation. It would be interesting; in a further study to determine if the down regulation and loss of nuclear expression of Gal-3 in colonic adenoma-carcinoma sequence is mirrored in either the progression of endometrial carcinoma in situ (ECIS) to UPSC or in the atypical hyperplasia-carcinoma sequence of EEC.

Stromal Gal-3 was identified in very few cases of EEC and UPSC. Therefore it was not possible to determine any association between Gal-3 expression and CD98 expression and in particular to determine if Gal-3 was acting as a ligand for CD98 in these tumours.

\subsubsection{Summary}

This immunohistochemical study has confirmed that CD98 and Gal-3 are present in both EEC and UPSC. We found significantly higher expression of CD98 in EEC compared to UPSC. This may be due to the known relationship of CD98 with $\beta 1$ integrin and indirectly of $\beta 1$ integrin with ER status. Despite the lower expression of CD98 in UPSC compared to EEC we did show increased expression of CD98 at the invasive edge of UPSC. This is may be due to increased requirement of invading UPSC cells for nutrients, alternately this may reflect cross linking of $\beta 1$ integrin and thus activation of $\beta 1$ integrin signalling and thus anchorage independent growth. There was no significant difference in Gal-3 expression between the tumour types, but Gal-3 expression did significantly decrease at the invasive edge of UPSC 
compared to the central viable part of the tumour. This decrease in Gal-3 expression also occurred to a non-significant level in EEC and was mirrored by a similar loss of nuclear expression of Gal-3. These findings raise the possibility that loss of nuclear expression of Gal-3 is associated with the development of invasive properties of these tumours. 


\section{Chapter 7}

\section{The Role of Matrix Metalloproteases -2, -7 and -9 in Uterine Papillary Serous Carcinoma and Endometrioid Endometrial Carcinoma}

\subsection{Introduction}

Endometrial carcinoma is the most common female genital malignancy in the western world \{Creasman, 2003\}. There are 2 main types of endometrial carcinomas: endometrioid endometrial carcinoma (EEC) (Type I) and nonendometrioid endometrial carcinomas (Type II) which mainly consist of uterine papillary serous carcinoma (UPSC), clear cell carcinoma and malignant mixed mesodermal tumour (carcinosarcoma). EEC accounts for approximately $80 \%$ of all endometrial carcinomas and usually arises from atypical hyperplasia of the endometrium \{Burton, 1998;Santin, 2002;Santin, 2003\}. It is associated with oestrogen excess. UPSC accounts for approximately $10 \%$ of cases of endometrial carcinoma \{Burton, 1998\} and makes up the majority of the Type II carcinomas. It is an aggressive tumour, usually occurring in elderly women and is thought to arise from endometrial intraepithelial carcinoma \{Wheeler, 2000;Ambros, 1995;Spiegel, 1995\}. It is not associated with either atypical hyperplasia or oestrogen status. Although UPSC comprises $10 \%$ of cases of endometrial carcinoma \{Burton, 1998$\}$ it accounts for a much smaller proportion of Stage I endometrial carcinomas \{Hendrickson, 1983;Bancher-Todesca, 1998;Dembo, 1985\}. It is upstaged at the time of surgery in $60 \%$ of cases \{Gehrig, 2001;Jeffrey, 1986;Kato, 1995;Goff, 1994;Dunton, 1991\}. Hui et al \{Hui, 2005\} examined a series of 40 cases of early uterine serous carcinoma. Nine of these were endometrial intraepithelial carcinoma 
and 31 were cases of Stage Ia UPSC. They demonstrated that only when the carcinoma was confined to an endometrial polyp, did the patients have good prognosis. It is possible that this aggressive behaviour could be explained by UPSC interacting with the extracellular matrix $(\mathrm{ECM})$ in a different way to EEC.

Liotta et al \{Liotta, 1986\} suggested a hypothetical model in which tumour invasion and metastasis resulted from repetition of three steps; adhesion of cancer cells to BM glycoproteins; degradation of BMs by specific proteolytic enzymes; and migration of cancer cells. This chapter focuses on degradation of the ECM.

The theory of tumour progression and heterogeneity is well established. Tumours are known to lose clonality early in development and generate subclones with varying characteristics from mutations. In theory only the selected subclone will possess the correct genetic characteristics in order to invade and metastasise.

\subsubsection{Matrix Metalloproteases}

Matrix metalloproteinases (MMPs), a family of zinc-dependent endoproteinases, are widely accepted to play a role in the invasion and metastasis of tumours $\{$ Chang, 2001;Vihinen, 2002\}. Under normal physiological conditions MMPs are expressed at a very low level in adult tissues, except in tissues that undergo remodelling such as cycling endometrium, normal breast tissue at the time of involution and the skin during wound healing \{Parks, 1999; Chang, 2001;Rodgers, 1993;Rodgers, 1994\}. Alteration of MMP expression has been implicated in a number of diseases such as rheumatoid arthritis, osteoarthritis, chronic wounds and cancer \{Chang, 2001;Vihinen, 2002;Stamenkovic, 2000;Parks, 1999;John, 2001;Jiang, 2002\}. Increased MMP expression and proteolytic degradation of ECM have been detected 
in a wide range of cancers, including those arising from the breast, colon, ovary and lung and MMP expression has been correlated with primary tumour growth and angiogenesis and also tumour invasion and metastasis \{Chang, 2001;Vihinen, 2002;Stamenkovic, 2000;John, 2001;Jiang, 2002;Kugler, 1999;Stetler-Stevenson, $1999\}$.

Based on their structure and substrate specificity MMPs are divided into several groups that include collagenases (MMP-1, MMP-3 and MMP-8), gelatinases (MMP2 and MMP-9), stromeolysins (MMP-3, MMP-10, MMP-11), matrilysins (MMP-7, MMP-26), metalloelastase (MMP-12) and membrane type matrix metalloproteinases (MT-MMPs) \{Chang, 2001;Vihinen, 2002;Stamenkovic, 2000;Parks, 1999;John, 2001; Jiang, 2002\}.

In the past it was generally accepted that cancer cells were responsible for producing MMPs in human tumours. However, this concept underwent revision in 1990 when Basset et al \{Basset, 1990\} reported that the stromal fibroblasts within tumours, rather than the tumour cells themselves, were responsible for the production of MMP-11 in human breast cancer. Since then MMPs-1, -2, -3, -9, and MT1-MMP mRNA has been shown by in situ hybridization to be primarily in fibroblasts especially in proximity to invading cancer cells in a variety of tumours including breast, colon, and ovary \{Polette, 1996;Nelson, 2000\}. In contrast, MMP-2 mRNA has been shown in prostatic cells rather than in fibroblasts $\{$ Still, 2000\}. In addition, immunohistochemistry (IHC) on a variety of human cancers has consistently demonstrated the localization of MMP protein in cancer cells. These data suggested that MMPs produced and secreted by stromal fibroblasts and inflammatory cells may bind to the cell membrane of cancer cells, thus giving them the proteases required for 
invasion. To support this, $\mathrm{Yu}$ et al $\{\mathrm{Yu}, 2000\}$ demonstrated that MMP-2 binds to the TIMP-2:MT-MMP complex on the cell surface and that MMP-9 binds to CD44. Olson et al \{Olson, 1998\} also showed that MMP-9 binds to type IV collagen on the cell surface, and Barmina et al \{Barmina, 1999\} demonstrated that MMP-13 binds to a receptor on the cell surface prior to endocytosis and lysosomal degradation. A complicating factor is that MMP expression appears to vary among host organ microenvironments and stromal MMPs may promote metastasis to one organ in preference to another. Therefore anti-metastatic effects based on MMP inhibition may be dependent on MMPs derived from specific organ microenvironments as well as tumour cells $\{$ Shiraga, 2002\}.

Although some MMPs are synthesised and secreted by cancer cells others are synthesised and secreted by stromal cells and then bind to the cell membrane of tumour cells \{Basset, 1990;Polette, 1996;Nelson, 2000;Still, 2000;Yu, 2000;Olson, 1998;Barmina, 1999\}. MMP expression appears to vary among host organ microenvironments and stromal MMPs may promote metastasis to one organ in preference to another. Therefore anti-metastatic effects based on MMP inhibition may be dependent on MMPs derived from specific organ microenvironments as well as tumour cells $\{$ Shiraga, 2002$\}$.

In female reproductive tract tissues, several MMPs and tissue inhibitory metalloproteases (TIMPs) are expressed, and there is an association with events such as menstruation, abnormal uterine bleeding, ovulation, embryo implantation, cervical ripening and parturition \{Martelli, 1993;Rodgers, 1994;Matrisian, 1992\}. 


\subsubsection{Matrix Metalloprotease 2 (MMP-2)}

ProMMP-2, the precursor to active MMP-2, is widely expressed in normal tissue and the MMP-2 gene has been described as a 'house-keeping' gene for its role in normal cellular processes \{Matrisian, 1994\}. In contrast, active MMP-2 is increased significantly in some neoplastic tissues and absent in most normal tissues. MMP-2 contributes to cell migration by a mechanism involving interaction with collagen $\{\mathrm{Xu}, 2005\}$. MMP-2 has been demonstrated in endometrial carcinoma cells $\{$ Misugi, $2005\}$.

Park et al \{Park, 2001\} demonstrated that synthesis and secretion of stromal MMP-2 was up regulated by $\beta$ oestradiol. In addition they showed that cells from an endometrial adenocarcinoma cell line invaded by recruiting MMP-2 secreted by endometrial stromal cells to their cell membrane and again, this was enhanced by the presence of $\beta$ oestradiol.

MMP-2 expression is used to help differentiate high grade from low-grade endometrial carcinoma \{Iurlaro, 1999; Liokumovich, 1999;Inoue, 1997\}.

\subsubsection{Matrix Metalloprotease 7 (MMP-7)}

Altered expression of MMP-7 has been demonstrated in EEC and increased expression of MMP-7 is associated with lymph node metastasis \{Yabushita, 2000;Ueno, 1999;Moser, 1999;Misugi, 2005\}. In colonic adenocarcinoma MMP-7 expression is known to be increased in tumour cells but not stromal cells \{Matrisian, 1994 \} and is associated with the risk of distant metastases and lymph node metastases \{Adachi, 1999;Newell, 1994\}. Matrisian et al \{Matrisian, 1994\} showed that induction of MMP-7 expression in tumour cells increased tumorigenicity and 
metastases, while inhibition of MMP-7 led to decreased metastasis \{Witty, 1994; Hasegawa, 1998\}. In addition, they found that MMP-7 expression appeared to be associated with a less aggressive phenotype, despite being involved in invasion. In contrast, Misugi et al $\{$ Misugi, 2005\} demonstrated that increased expression of MMP-7 was found in high grade endometrial carcinomas and this expression was also associated with tumour invasion and metastasis. Mylona et al \{Mylona, 2005\} detected MMP-7 expression in both tumour and stromal cells of breast carcinoma and showed a direct correlation of expression of MMP-7 with MMP-2 in the same tumour types.

Crawford et al \{Crawford, 1999\} described a positive correlation between nuclear $\beta$ catenin protein levels and MMP-7 transcripts in colonic carcinoma. They did not find any MMP-7 expression in cells that lacked $\beta$ catenin protein accumulation. In contrast, Mylona et al \{Mylona, 2005\} demonstrated an inverse correlation with nuclear $\beta$ catenin expression in breast carcinoma.

\subsubsection{Matrix Metalloprotease 9 (MMP-9)}

MMP-9 expression has been shown to be altered in EEC and increased expression is associated with myometrial invasion \{Yabushita, 2000;Ueno, 1999;Moser, 1999;Misugi, 2005\}. In addition, Park et al \{Park, 2001\} showed that synthesis and secretion of MMP-9 was down regulated by $\beta$ oestradiol. Cioppi et al \{Cioppi, 2004\} found that MMP-9 expression was higher in oestrogen receptor (ER) positive endometrial carcinomas, In addition MMP-9 has been shown to be useful in differentiating normal from sarcomatous endometrial stroma \{Iurlaro, 1999;Liokumovich, 1999;Inoue, 1997\}. 


\subsubsection{Matrix Metalloprotease 26 (MMP-26)}

MMP-26 and TIMP-4 expression is elevated in endometrial carcinomas with the highest expression correlating with deeper myometrial invasion and high grade tumours. There is no difference in expression between high-grade EEC and USPC \{Tunuguntla, 2003\}.

\subsubsection{Summary.}

Although UPSC comprises only $10 \%$ of endometrial carcinomas it accounts for a greater proportion of deaths. This chapter investigated the expression of MMPs-2, -7 and -9 in UPSC and EEC, and in particular to see if there was any difference in expression between the two tumour types which might explain the observed difference in aggressive behaviour. Previous studies have shown that the expression of MMPs -2 and -7 may be upregulated by $\beta$ oestradiol; therefore, we also examined the expression of ER and progesterone receptors (PR) in these tumours. $\beta$ catenin expression was studied in more detail in Chapter 5, but as studies on colon and breast carcinoma have shown both positive and negative correlations between the expression of $\beta$ catenin and MMP-7, any correlation between $\beta$ catenin expression and any of the MMPs was noted. It is well recognised that tumours are heterogeneous and examining the central part of the tumour alone may give misleading results as regards the invasiveness of the tumour as a whole. We used tissue microarrays (TMAs), to examine a relatively large number of cases, in order to be able to demonstrate differences in expression of these proteins between the viable central part of the tumour and the invasive edge in UPSC and EEC. 


\subsection{Materials and Methods}

\subsubsection{Tissues}

Tissues were obtained as described in "Materials and Methods", Section 2.1.

\subsubsection{Tissue Microarray}

The tissue microarray was constructed as described in "Materials and Methods", Section 2.2.

\subsubsection{Immunohistochemistry}

Immunohistochemistry was performed for MMPs $-2,-7$ and -9 and for ER and PR using the technique described in "Materials and Methods", Sections 2.3.3, 2.3.4, 2.3.5, 2.3.2 and 2.3.6 respectively.

\subsubsection{Scoring}

Scoring of antibody expression was assessed using the technique as described in "Materials and Methods", Section 2.4.

\subsubsection{Statistical Analysis}

Statistical analysis was performed as described in "Materials and Methods", Section 2.5 . 


\subsection{Results}

\subsubsection{Intertumoural Variability}

Significantly greater MMP-2 expression was present in EEC carcinoma cells compared to UPSC carcinoma cells $(\mathrm{p}<0.05$ Mann Whitney U Test, Table 7.1). There was no significant difference in MMP-2 expression between UPSC and EEC stromal cells. In addition, there was no significant difference in either carcinoma cell or stromal cell expression of MMP-7 between UPSC and EEC. Although there was no significant difference in MMP-9 expression between UPSC and EEC carcinoma cells, there was significantly greater MMP-9 expression seen in EEC stromal cells ( $\mathrm{P}$ $=0.001$, Mann Whitney U Test, Table 7.1). There was significantly greater expression of ER and PR in EEC compared to UPSC $(\mathrm{P}=0.033$ and $\mathrm{P}=0.02$ respectively Mann Whitney U Test, Table 7.1). Scatterplots and Pearson pairwise correlation coefficient did not show any direct or inverse correlation between $\beta$ catenin expression and MMPs-2, -7 or -9 , in either UPSC or EEC. 
Table 7.1. Expression of MMPs $-2,-7$ and -9 in the Viable Central Part of the Tumour in UPSC and EEC.

\begin{tabular}{|c|c|c|c|c|}
\hline \multirow[b]{2}{*}{ Antibody } & \multicolumn{2}{|l|}{ UPSC } & \multicolumn{2}{|l|}{ EEC } \\
\hline & Tumour cells & $\begin{array}{l}\text { Stromal } \\
\text { cells }\end{array}$ & Tumour cells & Stromal cells \\
\hline MMP-2 & $\begin{array}{l}0.53^{*} \\
\text { SD } 0.70\end{array}$ & $\begin{array}{l}1.68 \\
\text { SD } 0.67\end{array}$ & $\begin{array}{l}1.43^{*} \\
\text { SD } 0.97\end{array}$ & $\begin{array}{l}1.36 \\
\text { SD } 0.38\end{array}$ \\
\hline MMP-7 & $\begin{array}{l}1.81 \\
\text { SD } 0.55\end{array}$ & $\begin{array}{l}1.28 \\
\text { SD } 0.56\end{array}$ & $\begin{array}{l}1.77 \\
\text { SD } 0.48\end{array}$ & $\begin{array}{l}1.51 \\
\text { SD } 0.59\end{array}$ \\
\hline MMP-9 & $\begin{array}{l}0.90 \\
\text { SD } 0.73\end{array}$ & $\begin{array}{l}1.24^{+} \\
\text {SD } 0.73\end{array}$ & $\begin{array}{l}1.17 \\
\text { SD } 0.89\end{array}$ & $\begin{array}{l}2.44^{+} \\
\text {SD } 4.05\end{array}$ \\
\hline ER & $\begin{array}{l}4.38 \\
\text { SD } 2.46\end{array}$ & & $\begin{array}{l}\text { * } 5.85 \\
\text { SD } 3.00\end{array}$ & \\
\hline PR & $\begin{array}{l}0.96 \\
\text { SD } 1.70\end{array}$ & & $\begin{array}{c}4.67 \\
\text { SD } 3.42\end{array}$ & \\
\hline
\end{tabular}

*P $<0.05$ (Mann Whitney U Test)

${ }^{+} \mathrm{P}=0.001$ (Mann Whitney U Test)

${ }^{* *} \mathrm{P}=0,033$ (Mann Whitney U Test)

${ }^{* * *} \mathrm{P}=0.02$ (Mann Whitney U Test) 


\subsubsection{Intratumoural Variability}

\subsubsection{Uterine Papillary Serous Carcinoma}

There was significantly greater MMP-2 expression in carcinoma cells at the invasive edge compared to those in the viable central part of the tumour $(\mathrm{p}=0.045$, Wilcoxon Signed Ranks Test). However, there was no significant difference in expression of MMP-2 in stromal cells between the invasive edge and the viable central part of the tumour (Table 7.2). MMP-2 staining was present in both the cytoplasm and at the cell membrane (Figure 7.1).

There was no significant difference in either MMP-7 or MMP-9 expression between the viable central part of the tumour or the invasive edge in either carcinoma or stromal cells (Table 7.2). Staining of both MMP-7 and MMP-9 in both carcinoma and stromal cells was cytoplasmic and membranous (Figures 7.2 and 7.3 respectively).

There was significantly greater expression of MMP-2 in stromal cells compared to carcinoma cells in both the viable central part of the tumour and at the invasive edge $(\mathrm{p}<0.05$ and $\mathrm{p}=0.001$ respectively, Wilcoxon Signed Ranks Test, Table 7.3). In contrast there was significantly less expression of MMP-7 in the stromal cells compared to the carcinoma cells in both the viable central part of the tumour and at the invasive edge $(p<0.05$ and $p=0.001$ respectively, Wilcoxon Signed Ranks Test, Table 7.3). There was significantly less MMP-9 expression in carcinoma cells compared to stromal cells in the viable central part of the tumour $(p=0.02$, Wilcoxon Signed Ranks Test, Table 7.3), but there was no significant difference in MMP-9 expression between these cells at the invasive edge. 
Table 7.2. Expression of MMPs $-2,-7$ and -9 in the Viable Central Part of the Tumour and at the Invasive Edge.

\begin{tabular}{|c|c|c|c|c|}
\hline \multirow[b]{2}{*}{ Antibody } & \multicolumn{2}{|l|}{ UPSC } & \multicolumn{2}{|l|}{ EEC } \\
\hline & Tumour & $\begin{array}{l}\text { Invasive } \\
\text { Edge }\end{array}$ & Tumour & $\begin{array}{l}\text { Invasive } \\
\text { Edge }\end{array}$ \\
\hline MMP-2 & $\begin{array}{l}0.53^{*} \\
\text { SD } 0.70\end{array}$ & $\begin{array}{l}0.79^{*} \\
\text { SD } 0.46\end{array}$ & $\begin{array}{l}1.43 \\
\text { SD } 0.97\end{array}$ & $\begin{array}{l}1.69 \\
\text { SD } 0.58\end{array}$ \\
\hline $\begin{array}{l}\text { MMP-2 } \\
\text { Stroma }\end{array}$ & $\begin{array}{l}1.68 \\
\text { SD } 0.68\end{array}$ & $\begin{array}{l}1.54 \\
\text { SD } 0.73\end{array}$ & $\begin{array}{l}1.42 \\
\text { SD } 0.52\end{array}$ & $\begin{array}{l}1.46 \\
\text { SD } 0.94\end{array}$ \\
\hline MMP-7 & $\begin{array}{l}1.81 \\
\text { SD } 0.55\end{array}$ & $\begin{array}{l}1.69 \\
\text { SD } 0.85\end{array}$ & $\begin{array}{l}1.77 \\
\text { SD } 0.48\end{array}$ & $\begin{array}{l}1.61 \\
\text { SD } 0.57\end{array}$ \\
\hline $\begin{array}{l}\text { MMP-7 } \\
\text { Stroma }\end{array}$ & $\begin{array}{l}1.27 \\
\text { SD } 0.83\end{array}$ & $\begin{array}{l}1.21 \\
\text { SD } 0.75\end{array}$ & $\begin{array}{l}1.52 \\
\text { SD } 0.43\end{array}$ & $\begin{array}{l}1.51 \\
\text { SD } 0.56\end{array}$ \\
\hline MMP-9 & $\begin{array}{l}0.90 \\
\text { SD } 0.73\end{array}$ & $\begin{array}{l}1.08 \\
\text { SD } 0.84\end{array}$ & $\begin{array}{l}1.14 \\
\text { SD } 0.89\end{array}$ & $\begin{array}{l}1.09 \\
\text { SD } 0.96\end{array}$ \\
\hline $\begin{array}{l}\text { MMP-9 } \\
\text { Stroma }\end{array}$ & $\begin{array}{l}1.24 \\
\text { SD } 0.99\end{array}$ & $\begin{array}{l}1.22 \\
\text { SD } 0.76\end{array}$ & $\begin{array}{l}2.51 \\
\text { SD } 1.08\end{array}$ & $\begin{array}{l}1.76 \\
\text { SD } 0.72\end{array}$ \\
\hline
\end{tabular}

SD Standard Deviation

* $\mathrm{P}=0.045$ (Wilcoxon Signed Ranks Test) 
Table 7.3. Variation in Matrix Metalloproteinase Expression between

Tumour and Stromal cells in UPSC.

\begin{tabular}{|c|c|c|c|c|}
\hline \multirow[b]{2}{*}{ Antibody } & \multicolumn{2}{|l|}{ Main Tumour } & \multicolumn{2}{|l|}{ Invasive Edge } \\
\hline & Tumour cells & $\begin{array}{l}\text { Stromal } \\
\text { cells }\end{array}$ & Tumour cells & Stromal cells \\
\hline MMP-2 & $\begin{array}{l}0.53^{*} \\
\text { SD } 0.70\end{array}$ & $\begin{array}{l}1.68^{*} \\
\text { SD } 0.68\end{array}$ & $\begin{array}{l}0.79^{+} \\
\text {SD } 0.46\end{array}$ & $\begin{array}{l}1.54^{+} \\
\text {SD } 0.73\end{array}$ \\
\hline MMP-7 & $\begin{array}{l}1.81^{* *} \\
\text { SD } 0.55\end{array}$ & $\begin{array}{l}1.27^{* *} \\
\text { SD } 0.83\end{array}$ & $\begin{array}{l}1.69^{++} \\
\text {SD } 0.85\end{array}$ & $\begin{array}{l}1.21^{++} \\
\text {SD } 0.75\end{array}$ \\
\hline MMP-9 & $\begin{array}{l}0.90^{* * *} \\
\text { SD } 0.73\end{array}$ & $\begin{array}{l}1.24^{* * *} \\
\text { SD } 0.99\end{array}$ & $\begin{array}{l}1.08 \\
\text { SD 0.84 }\end{array}$ & $\begin{array}{l}1.22 \\
\text { SD } 0.76\end{array}$ \\
\hline
\end{tabular}

SD Standard Deviation

${ }^{*} \mathrm{P}<0.05$ (Wilcoxon Signed Ranks Test)

${ }^{+} \mathrm{P}=0.001$ (Wilcoxon Signed Ranks Test)

**P $<0.05$ (Wilcoxon Signed Ranks Test)

${ }^{++} \mathrm{P}=0.001$ (Wilcoxon Signed Ranks Test)

$* * * \mathrm{P}=0.02$ (Wilcoxon Signed Ranks Test) 


\section{Figure 7.1}

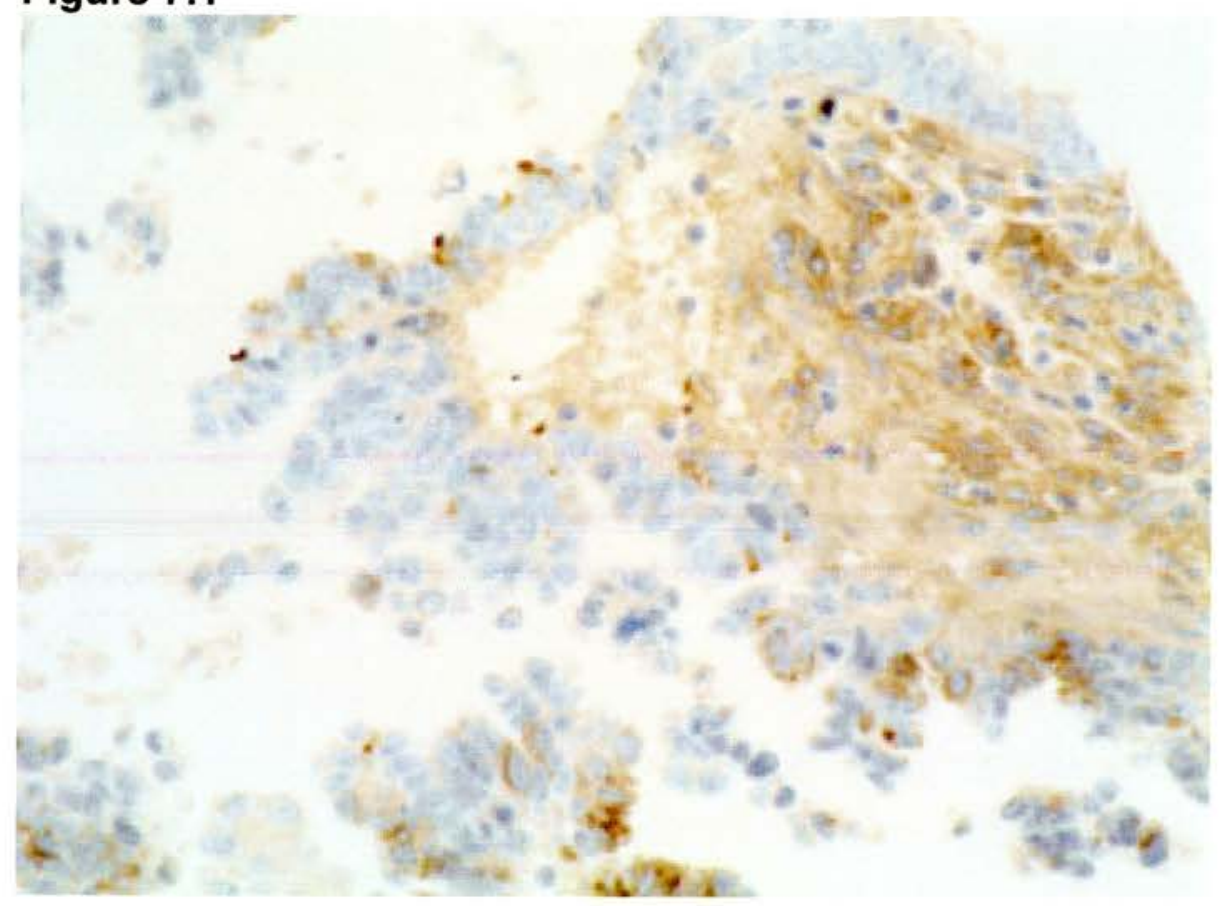

\section{Figure 7.2}

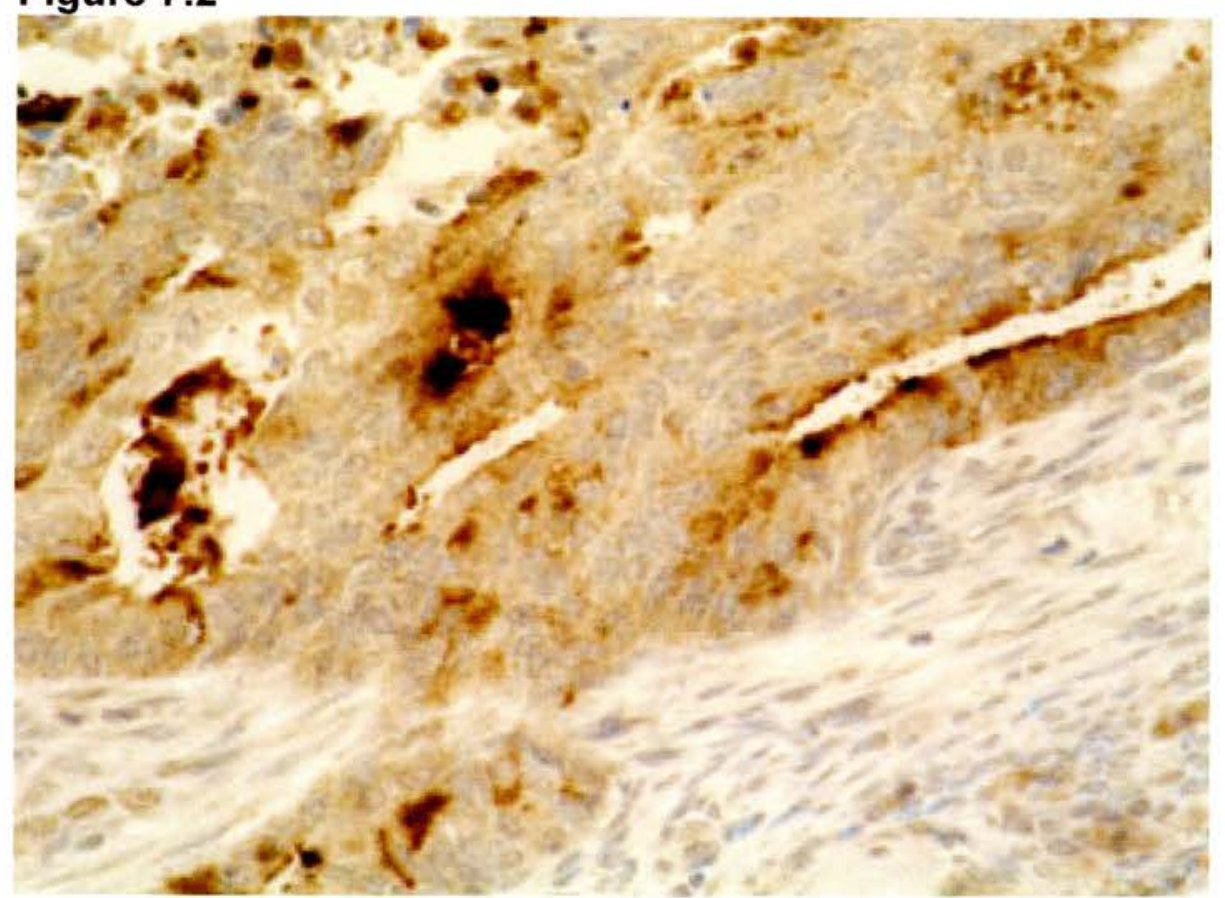

Figure 7.1. Expression of MMP-2 predominantly in UPSC stromal cells (Magnificationx20).

Figure 7.2. Expression of MMP-7 in both carcinoma and stromal cells of UPSC (Magnificationx20). 
Figure 7.3

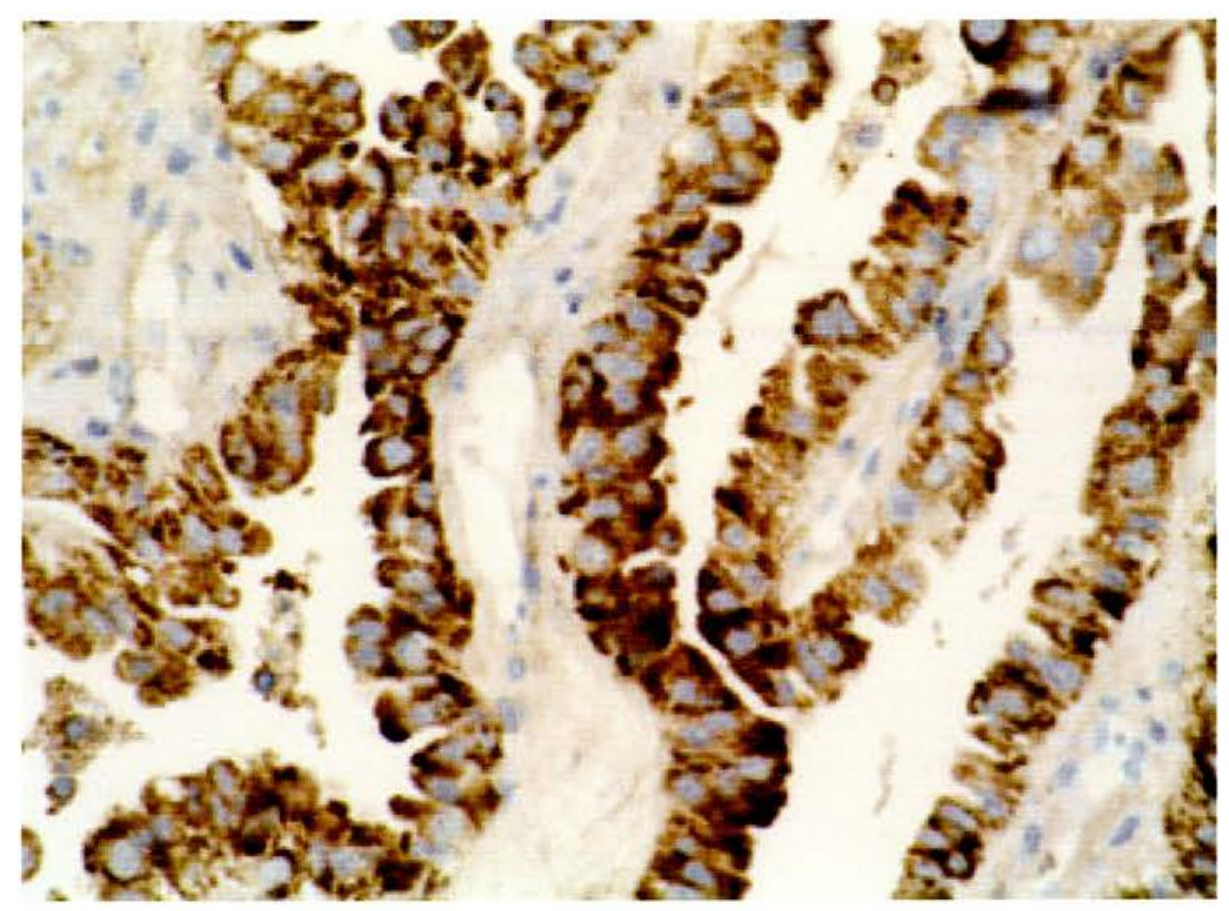

Figure 7.3. Expression of MMP-9 predominantly in UPSC carcinoma cells (Magnificationx20). 


\subsubsection{Endometrioid Endometrial Carcinoma}

No significant differences in expression of any of the proteins (MMPs-2, -7 or-9) were noted in either carcinoma cells or stromal cells at the invasive edge compared to those in the viable central part of the tumour (Table 7.2). Expression of all the MMPs was both cytoplasmic and membranous (Figures 7.4, 7.5 and 7.6 respectively).

There was no significant difference in expression of MMP-2 between carcinoma and stromal cells in both the viable central part of the tumour and at the invasive edge (Table 7.4). There was significantly greater expression of MMP-7 in carcinoma cells compared with stromal cells in the viable central part of the tumour $(\mathrm{p}=0.018$, Wilcoxon Signed Ranks Test, Table 7.4), but there was no significant difference in expression between the cell types at the invasive edge. Significantly greater expression of MMP-9 was noted in stromal cells compared to carcinoma cells in both the viable central part of the tumour and at the invasive edge $(p=0.011$ and $p=$ 0.012 respectively, Wilcoxon Signed Ranks Test, Table 7.4). 
Table 7.4. Variation in Matrix Metalloproteinase Expression between Tumour and Stromal cells in Endometrioid Endometrial Carcinoma.

\begin{tabular}{|c|c|c|c|c|}
\hline \multirow[t]{2}{*}{ Antibody } & \multicolumn{2}{|l|}{ Main Tumour } & \multicolumn{2}{|l|}{ Invasive Edge } \\
\hline & Tumour cells & $\begin{array}{l}\text { Stromal } \\
\text { cells }\end{array}$ & Tumour cells & Stromal cells \\
\hline MMP-2 & $\begin{array}{l}1.43 \\
\text { SD } 0.97\end{array}$ & $\begin{array}{l}1.42 \\
\text { SD } 0.52\end{array}$ & $\begin{array}{l}1.69 \\
\text { SD } 0.58\end{array}$ & $\begin{array}{l}1.46 \\
\text { SD } 0.94\end{array}$ \\
\hline MMP-7 & $\begin{array}{l}1.77^{*} \\
\text { SD } 0.48\end{array}$ & $\begin{array}{l}1.52^{*} \\
\text { SD } 0.43\end{array}$ & $\begin{array}{l}1.61 \\
\text { SD } 0.57\end{array}$ & $\begin{array}{l}1.51 \\
\text { SD } 0.56\end{array}$ \\
\hline MMP-9 & $\begin{array}{l}1.14^{* *} \\
\text { SD } 0.89\end{array}$ & $\begin{array}{l}2.51^{* *} \\
\text { SD } 1.08\end{array}$ & $\begin{array}{l}1.09^{+} \\
\text {SD } 0.96\end{array}$ & $\begin{array}{l}1.76^{+} \\
\text {SD } 0.72\end{array}$ \\
\hline
\end{tabular}

SD Standard Deviation

${ }^{*} \mathrm{P}=0.018$ (Wilcoxon Signed Ranks Test) $\quad{ }^{+} \mathrm{P}=0.012$ (Wilcoxon Signed Ranks Test)

${ }^{* *} \mathrm{P}=0.011$ (Wilcoxon Signed Ranks Test) 
Figure 7.4

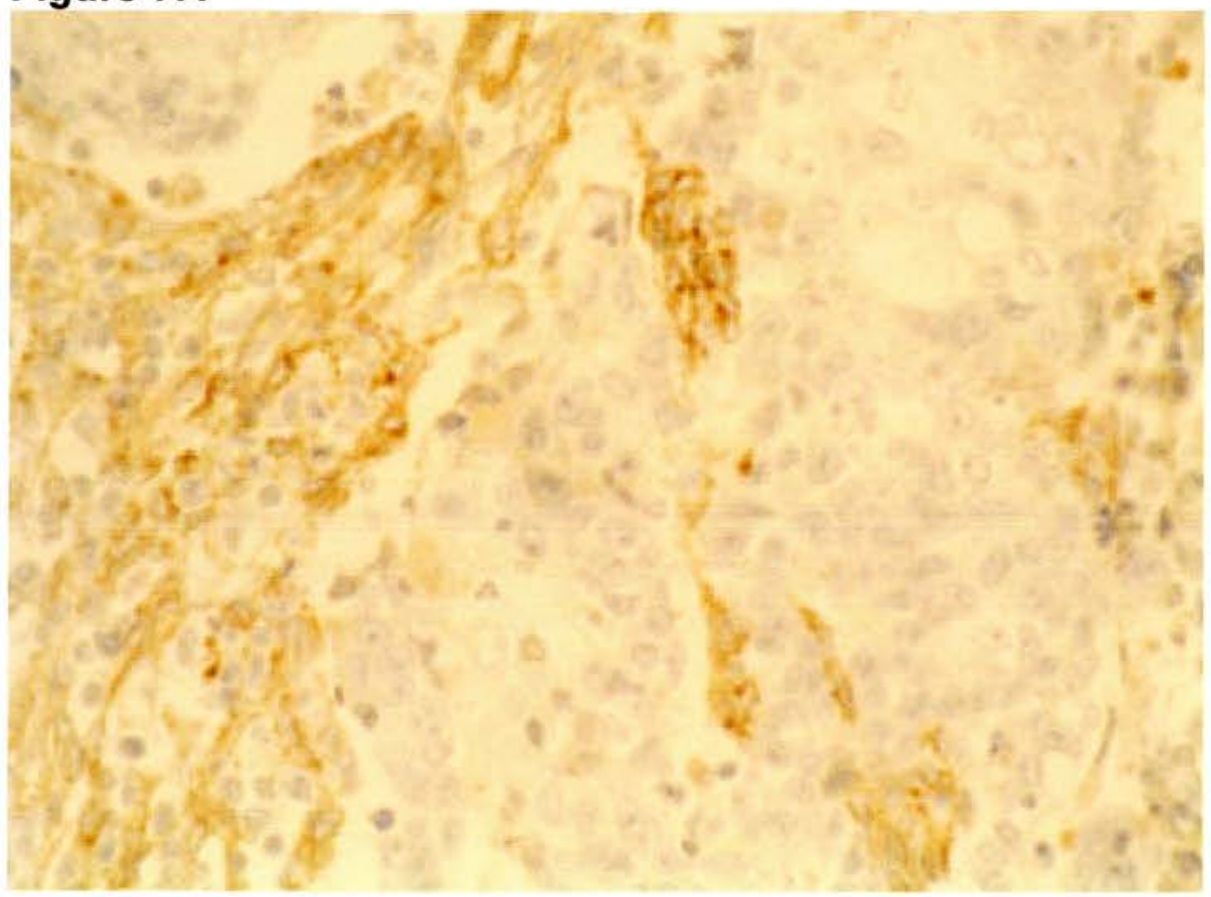

Figure 7.5

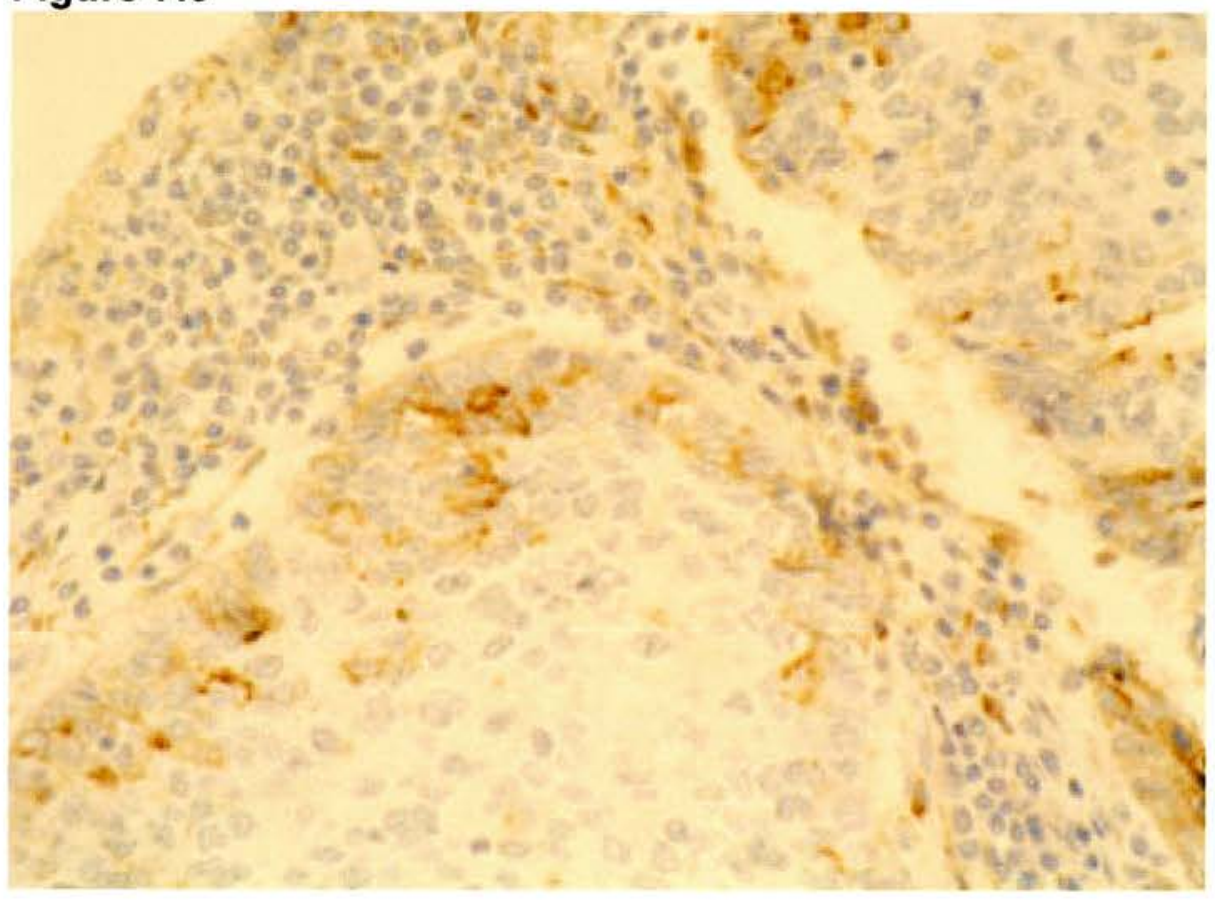

Figure 7.4. Expression of MMP-2 predominantly in EEC stromal cells (Magnification $\times 40)$.

Figure 7.5. Expression of MMP-7 in both carcinoma and stromal cells of EEC (Magnificationx40). 
Figure 7.6

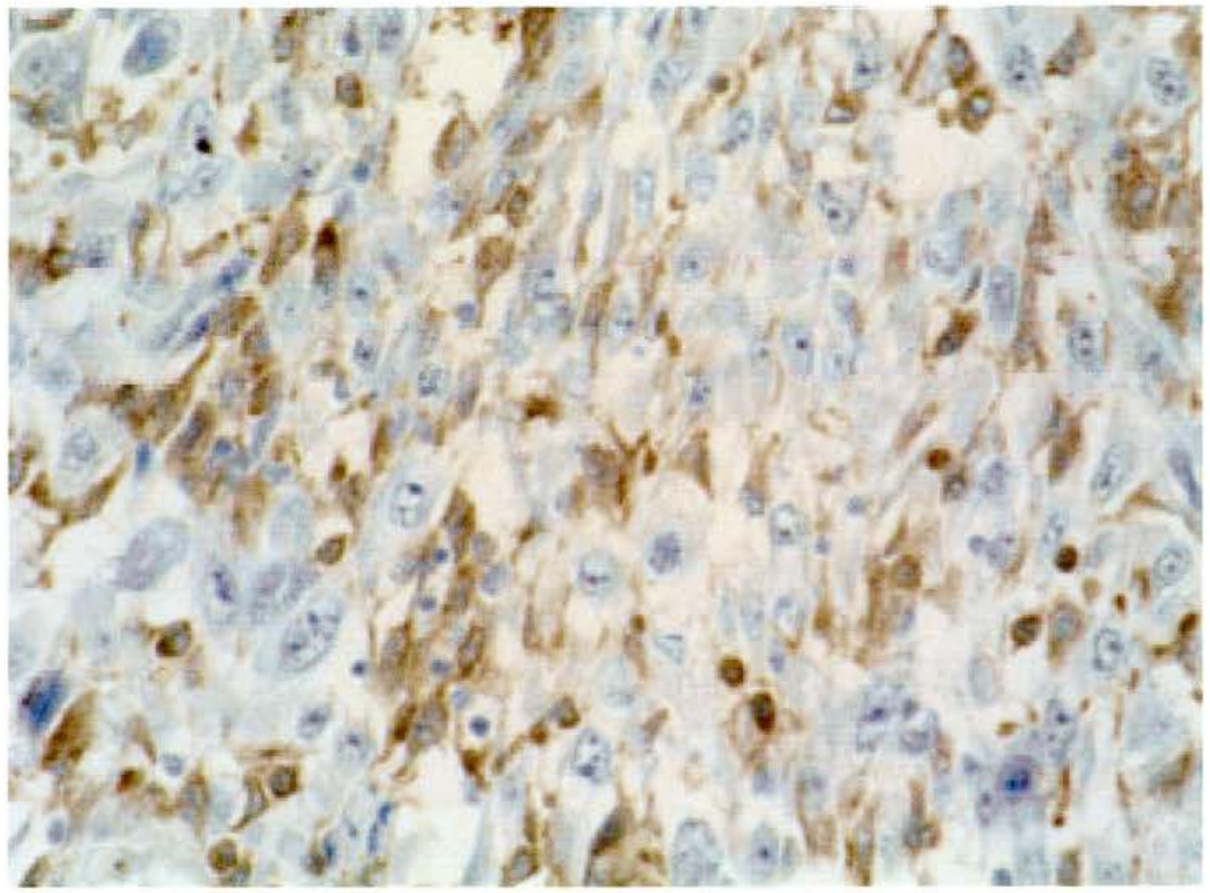

Figure 7.6. Expression of MMP-9 predominantly in EEC carcinoma cells (Magnificationx40). 


\subsection{Discussion}

UPSC is an aggressive endometrial tumour with a notable propensity for lymphovascular space invasion and a poor prognosis. This chapter aimed to determine if MMPs had a role in the increased invasiveness of UPSC in comparison to EEC. We confirmed the findings of Misugi et al \{Misugi, 2005\} and MorenoBueno et al \{Moreno-Bueno, 2003\} and demonstrated MMPs $-2,-7$ and -9 to be present in both UPSC and EEC.

In addition, this chapter looked for differences in expression of these proteins between the different histological tumour types and within the tumours (the viable central part of the tumour and at the invasive edge). We demonstrated MMP-2 expression in both EEC and UPSC carcinoma cells, confirming the findings of Park et al \{Park, 2001\}. Park et al \{Park, 2001\} demonstrated that synthesis and secretion of stromal MMP- 2 was up regulated by $\beta$ oestradiol. In addition they showed that the invasiveness of cells from an endometrial adenocarcinoma cell line was enhanced by recruitment of MMP-2 secreted by endometrial stromal cells to their cell membrane, which was further enhanced by the presence of $\beta$ oestradiol.

The finding of significantly higher MMP-2 expression in EEC carcinoma cells compared to those in UPSC may partly reflect the different biological properties of these tumours. EEC is a tumour which usually arises on a background of oestrogen excess, which commonly expresses ER and can produce intratumoural oestrogen using aromatase \{Segawa, 2005\}. On the other hand, UPSC usually arises from atrophic endometrium, is generally ER negative and does not produce intratumoural oestrogen. Park et al \{Park, 2001\} demonstrated that $\beta$ oestradiol stimulated MMP-2 
secretion in adenocarcinoma cell lines, therefore it is possible that the differential secretion may be due to increased intratumoural oestrogen in EEC compared to UPSC. We found significantly higher ER and PR positivity in our EEC cases compared to UPSC.

We found similar levels of MMP-2 in EEC carcinoma and stromal cells and although there was increased expression of MMP-2 in carcinoma cells at the invasive edge of the tumour, this was not statistically significant. However, we did find a significantly higher level of MMP-2 in UPSC stromal cells compared with carcinoma cells both in the viable central part of the tumour and at the invasive edge. In addition, the UPSC carcinoma cells showed a significant increase in MMP-2 expression at the invasive edge compared to the viable central part tumour. MMP-2 is synthesised and secreted by stromal cells and then binds to the TIMP-2:MT-MMP complex on the carcinoma cell surface $\{\mathrm{Yu}, 2000\}$; this difference in expression raises the possibility that the invasive subclone of UPSC uses increased carcinoma cell binding of MMP-2 to equip the cells with the proteases required for invasion

We did not demonstrate any intertumoural or intratumoural differences in expression of MMP-7. There was significantly more expression of MMP-7 in carcinoma cells compared to stromal cells in both UPSC and EEC. Our findings are in keeping with the published literature that MMP-7 is generally expressed in tumours \{Yabushita, 2000;Ueno, 1999;Moser, 1999;Misugi, 2005\}. As this study examined high grade endometrial cancers only we could not confirm or refute the findings of Misugi et al 
\{Misugi, 2005\}, who showed differential expression of MMP-7 between the different grades of EEC.

Crawford et al \{Crawford, 1999\} described a positive correlation between nuclear $\beta$ catenin protein levels and MMP-7 transcripts in colonic carcinoma. They did not find any MMP-7 expression in cells that lacked $\beta$ catenin protein accumulation. Mylona et al $\{$ Mylona, 2005\} demonstrated an inverse correlation with nuclear $\beta$ catenin expression in breast carcinoma. We did not demonstrate any inverse or direct correlation with $\beta$ catenin expression and any of the MMPs. This may be due to the relatively small numbers of cases involved. Alternately this may be because the studies that demonstrated either inverse or direct correlations were focussed on colonic carcinoma where $\beta$ catenin expression is nuclear, reflecting mutation in the $\beta$ catenin gene and the studies by Mylona et al $\{$ Mylona, 2005\} in breast carcinoma also focussed on $\beta$ catenin mutation.

We found a significantly higher expression of MMP-9 in EEC compared to UPSC and also a significantly higher expression of MMP-9 in the stromal cells compared to the carcinoma cells in the viable central part of the tumour in both EEC and UPSC. In addition there was significantly higher expression of MMP-9 in stromal cells compared to carcinoma cells at the invasive edge of the EEC cases. However, there was no significant difference in MMP-9 expression between the invasive edge stromal cells and carcinoma cells in UPSC. MMP-9 is normally synthesised and secreted by stromal cells and binds to CD44 on carcinoma cells $\{\mathrm{Yu}, 2000\}$. The loss of this differential expression between carcinoma cells and stromal cells at the 
invasive edge of UPSC cases may indicate that MMP-9 synthesis and secretion by stromal cells is unchanged at the invasive edge of the tumour, but binding of MMP-9 by the invading carcinoma cells is increased, perhaps enhancing their invasive potential.

Hui et al \{Hui, 2005\} demonstrated differences in outcome between patients with Stage Ia UPSC arising in a polyp and in the normal endometrium. In view of our results showing differences in expression of MMPs -2 and -9 between the main part of the tumour and the invasive edge, it would be interesting to more closely examine cases of endometrial intraepithelial carcinoma and Stage Ia UPSC.

\subsubsection{Summary.}

In summary this immunohistochemical study has confirmed that MMPs- $2,-7$ and -9 are present in endometrial carcinomas and specifically in both EEC and UPSC. We also confirmed that our UPSC group had significantly lower ER and PR expression. We found significantly higher expression of MMPs -2 and -9 in EEC compared to UPSC. This may be due to the known relationship of these MMPs with oestrogen status. Despite the lower expression of MMP-2 and MMP-9 in UPSC compared to EEC we did show increased expression of MMP-2 and MMP-9 by carcinoma cells at the invasive edge of UPSC. This is probably due to increased binding of MMPs secreted by the stromal cells to carcinoma cells. This difference in staining pattern between the viable central part of the tumour and the invasive edge supports the theory of an invasive subclone of UPSC acquiring activated proteases in order to invade. 


\section{Summary of Thesis}

Despite having been described as a distinct clinical entity for over twenty years, uterine papillary serous carcinoma (UPSC) remains a management challenge for the multidisciplinary team due to the paucity of understanding on the epidemiology, pathogenesis, natural history and optimal treatment strategies for this important and aggressive variant of endometrial adenocarcinoma. The aim of this thesis is to specifically focus on molecular changes in UPSC, which might account for its aggressive behaviour and pattern of spread and to add to the growing volume of literature on UPSC. This thesis briefly describes the current literature on UPSC and demonstrates that although in the past the microscopic and clinical similarities of UPSC to ovarian serous papillary carcinoma (OSPC) led to some clinicians and pathologists to think that these tumours were biologically similar, they are actually quite distinct. There are a wide number of adjuvant treatment options open to patients with UPSC; the majority of which have only been formally tested on patients with OSPC.

The tissue microarray (TMA) validation study (Chapter 3) shows that the results of immunohistochemical staining of TMAs made from endometrial carcinoma are equivalent to those from whole sections of the same tumour. The ideal number of cores sampled should be 2 to 3 in order to decrease the variation seen in heterogeneous tumours, and also to increase the likelihood of an informative section in those tumours, which may be focal, infiltrative or scanty when present in a pipelle specimen. In addition, this chapter demonstrates that TMA use can be extended to include other high-grade endometrial carcinomas and antibodies with uncertain 
patterns of expression. Chapter 4 describes an audit of the pathological diagnosis and management of UPSC in South East Scotland. This confirms the poor prognosis of patients with UPSC and shows that in even those cases where the tumour only comprises $5 \%$ UPSC, the poor prognosis is conferred, regardless of the additional tumour type. This audit raises awareness for the need of accurate and complete surgical staging at a local level. Despite the poor response to chemoradiotherapy identified by this audit, local treatments for patients with UPSC have not altered, as patient numbers are small with a limited follow up time. This audit contributed to the increasing local, national and international awareness of the need to invest in randomised controlled trials on UPSC.

The remaining chapters focus on molecules involved in three steps associated with invasion. Chapter 5 examines the initial step in invasion where tumour cells decrease their homotypic binding in order to detach from one another and to invade. Therefore the expression of the cell adhesion molecules $\mathrm{E}$ cadherin, $\mathrm{P}$ cadherin and $\beta$ catenin in UPSC is determined and this expression is compared with high-grade endometrioid endometrial carcinoma (EEC). This chapter confirms that E cadherin, $\mathrm{P}$ cadherin and $\beta$ catenin are expressed in high-grade endometrial carcinoma and that there is differential E cadherin and P cadherin expression between UPSC and EEC. This may in part be due to the important role of oestrogen in the development and progression of EEC compared to UPSC. In addition, decreased expression of E cadherin and P cadherin between the viable central part of the tumour and the invasive edge is demonstrated.

The second step in invasion, after cells have dissociated from each other, is the attachment of tumour cells to molecules in the extracellular matrix (ECM). CD98 
and Galectin-3 (Gal-3) were selected for this chapter (Chapter 6). CD98 was chosen due to the relatively recent discovery of its association with $\beta 1$ integrin and Gal-3 was chosen as it has been identified as a possible ligand of CD98. This chapter confirms that CD98 and Gal-3 are present in both EEC and UPSC. There was significantly higher expression of CD98 in EEC compared to UPSC. This may be due to the known relationship of CD98 with $\beta 1$ integrin and indirectly $\beta 1$ integrin with ER status. Despite the lower expression of CD98 in UPSC compared to EEC there was increased expression of CD98 at the invasive edge of UPSC. This is may be due to increased requirement of invading UPSC cells for nutrients, or it may reflect cross linking of $\beta 1$ integrin and thus activation of $\beta 1$ integrin signalling and anchorage independent growth. There was no significant difference in Gal-3 expression between the tumour types, but Gal-3 expression did significantly decrease at the invasive edge of UPSC compared to the central viable part of the tumour. This decrease in Gal-3 expression also occurred to a non-significant level in EEC and was mirrored by a similar loss of nuclear expression of Gal-3. These findings raise the possibility that loss of nuclear expression of Gal-3 is associated with the development of invasive properties of these tumours.

The final step in invasion examined in this thesis is degradation of the ECM. Chapter 7 confirms that MMPs-2, -7 and -9 are present in high grade endometrial carcinoma. It also confirms that the UPSC group had significantly lower oestrogen receptor (ER) and progesterone receptor (PR) expression. Expression of MMPs-2 and -9 was significantly higher in EEC compared to UPSC. This may be due to the known relationship of these MMPs with oestrogen status. Despite the lower expression of MMP-2 and MMP-9 in UPSC compared to EEC, expression of MMP-2 and MMP-9 
by carcinoma cells at the invasive edge of UPSC was increased. This is probably due to increased binding to carcinoma cells of MMPs secreted by the stromal cells. The difference in staining pattern of $\mathrm{E}$ cadherin, $\mathrm{P}$ cadherin, $\beta$ catenin, $\mathrm{CD} 98$ and MMPs2 and -9 , between the viable central part of the tumour and the invasive edge supports the theory of a subclone of UPSC acquiring the properties necessary to invade.

In conclusion, this thesis has examined the role of molecules involved in some of the important steps involved in invasion; namely loss of cell-cell adhesion (E-cadherin, $\mathrm{P}$ cadherin and $\beta$ catenin), attachment to the ECM (CD98 and Gal-3) and degradation of the ECM (MMPs -2, -7 and -9). This demonstrated differences in expression of these molecules, not only between the central viable part of the tumour and the invasive edge but also between UPSC and EEC. The presence of intratumoural variation supports the theory that tumours become increasingly heterogenous with subclones developing different properties necessary for invasion and metastasis. The intertumoural variation emphasises the influence of oestrogen and therefore the oestrogen receptor status of the tumours as E cadherin, $\beta$ catenin, CD98 and MMPs 2 and -9 , are either directly or indirectly altered by oestrogen and, apart from E cadherin which was decreased, the other molecules all showed higher expression in EEC compared to UPSC. The results are consistent with the important role that oestrogen status plays in the development of EEC and it is possible that the lack of responsiveness of UPSC to oestrogen plays a role in its aggressive behaviour.

These data contribute to the growing body of literature on UPSC, and addresses diagnostic and treatment uncertainties for the pathology, surgical and oncological teams. 


\section{Reference List}

Aaltomaa S, Lipponen P, Ala-Opas M, Eskelinen M, Kosma VM. Alpha-catenin expression has prognostic value in local and locally advanced prostate cancer. $\mathrm{Br} \mathrm{J}$ Cancer 1999; 80(3-4):477-482.

Abeler VM, Kjorstad KE. Serous papillary carcinoma of the endometrium: a histopathological study of 22 cases.Gynecol Oncol 1990; 39(3):266-271.

Adachi Y, Yamamoto H, Itoh F, Hinoda Y, Okada Y, Imai K. Contribution of matrilysin (MMP-7) to the metastatic pathway of human colorectal cancers. Gut 1999; 45(2):252-258.

Akahani S, Nangia-Makker P, Inohara H, Kim HR, Raz A. Galectin-3: a novel antiapoptotic molecule with a functional BH1 (NWGR) domain of Bcl-2 family. Cancer Res 1997; 57(23):5272-5276.

Al Mehdi AB, Tozawa K, Fisher AB, Shientag L, Lee A, Muschel RJ. Intravascular origin of metastasis from the proliferation of endothelium-attached tumor cells: a new model for metastasis. Nat Med 2000; 6(1):100-102.

Ambros RA, Sherman ME, Zahn CM, Bitterman P, Kurman RJ. Endometrial intraepithelial carcinoma: a distinctive lesion specifically associated with tumors displaying serous differentiation.Hum Pathol 1995; 26(11):1260-1267. Arlt MF, Herzog TJ, Mutch DG, Gersell DJ, Liu H, Goodfellow PJ. Frequent deletion of chromosome $1 p$ sequences in an aggressive histologic subtype of endometrial cancer. Hum Mol Genet 1996; 5(7):1017-1021.

Bachelot T, Ray-Coquard I, Menetrier-Caux C, Rastkha M, Duc A, Blay JY. Prognostic value of serum levels of interleukin 6 and of serum and plasma levels of 
vascular endothelial growth factor in hormone-refractory metastatic breast cancer patients.Br J Cancer 2003; 88(11):1721-1726.

Bancher-Todesca D, Neunteufel W, Williams KE, Prainsack D, Breitenecker G, Friedlander ML et al. Influence of postoperative treatment on survival in patients with uterine papillary serous carcinoma. Gynecol Oncol 1998; 71(3):344-347.

Barakat RR, Wong G, Curtin JP, Vlamis V, Hoskins WJ. Tamoxifen use in breast cancer patients who subsequently develop corpus cancer is not associated with a higher incidence of adverse histologic features. Gynecol Oncol 1994; 55(2):164-168.

Barmina OY, Walling HW, Fiacco GJ, Freije JM, Lopez-Otin C, Jeffrey JJ et al. Collagenase- 3 binds to a specific receptor and requires the low density lipoprotein receptor-related protein for internalization.J Biol Chem 1999; 274(42):30087-30093.

Barondes SH, Cooper DN, Gitt MA, Leffler H. Galectins. Structure and function of a large family of animal lectins.J Biol Chem 1994; 269(33):20807-20810.

Basset P, Bellocq JP, Wolf C, Stoll I, Hutin P, Limacher JM et al. A novel metalloproteinase gene specifically expressed in stromal cells of breast carcinomas. Nature 1990; 348(6303):699-704.

Battifora H. The multitumor (sausage) tissue block: novel method for immunohistochemical antibody testing.Lab Invest 1986; 55(2):244-248.

Bauer R, Hein R, Bosserhoff AK. A secreted form of P-cadherin is expressed in malignant melanoma.Exp Cell Res 2005; 305(2):418-426.

Becker K, Pancoska P, Concin N, Vanden Heuvel K, Slade N, Fischer M et al. Patterns of $\mathrm{p} 73 \mathrm{~N}$-terminal isoform expression and p53 status have prognostic value in gynaecological cancers. Int J Oncol 2006; 29(4):889-902. 
Behrens J, Jerchow BA, Wurtele M, Grimm J, Asbrand C, Wirtz R et al. Functional interaction of an axin homolog, conductin, with beta-catenin, APC, and GSK3beta. Science 1998; 280(5363):596-599.

Bellone S, Watts K, Cane' S, Palmieri M, Cannon MJ, Burnett A et al. High serum levels of interleukin- 6 in endometrial carcinoma are associated with uterine serous papillary histology, a highly aggressive and chemotherapy-resistant variant of endometrial cancer. Gynecol Oncol 2005; 98(1):92-98.

Birchmeier W, Weidner KM, Behrens J. Molecular mechanisms leading to loss of differentiation and gain of invasiveness in epithelial cells.J Cell Sci Suppl 1993; 17:159-164.

Birdsall B, Feeney J, Burdett ID, Bawumia S, Barboni EA, Hughes RC. NMR solution studies of hamster galectin-3 and electron microscopic visualization of surface-adsorbed complexes: evidence for interactions between the $\mathrm{N}$ - and Cterminal domains.Biochemistry 2001; 40(15):4859-4866.

Biron-Shental T, Drucker L, Altaras M, Bernheim J, Fishman A. High incidence of BRCA 1-2 germline mutations, previous breast cancer and familial cancer history in Jewish patients with uterine serous papillary carcinoma. Eur J Surg Oncol , 1-4. 284-2006.

Blay JY, Negrier S, Combaret V, Attali S, Goillot E, Merrouche Y et al. Serum level of interleukin 6 as a prognosis factor in metastatic renal cell carcinoma. Cancer Res $1992 ; 52(12): 3317-3322$.

Bohm J, Niskanen L, Kiraly K, Kellokoski J, Eskelinen M, Hollmen S et al. Expression and prognostic value of alpha-, beta-, and gamma-catenins in differentiated thyroid carcinoma.J Clin Endocrinol Metab 2000; 85(12):4806-4811. 
Boise LH, Gonzalez-Garcia M, Postema CE, Ding L, Lindsten T, Turka LA et al. bcl-x, a bcl-2-related gene that functions as a dominant regulator of apoptotic cell death.Cell 1993; 74(4):597-608.

Bokhman JV. Two pathogenetic types of endometrial carcinoma. Gynecol Oncol $1983 ; 15(1): 10-17$.

Bookman MA, Malmstrom H, Bolis G, Gordon A, Lissoni A, Krebs JB et al. Topotecan for the treatment of advanced epithelial ovarian cancer: an open-label phase II study in patients treated after prior chemotherapy that contained cisplatin or carboplatin and paclitaxel.J Clin Oncol 1998; 16(10):3345-3352.

Bozdogan O, Atasoy P, Erekul S, Bozdogan N, Bayram M. Apoptosis-related proteins and steroid hormone receptors in normal, hyperplastic, and neoplastic endometrium. Int J Gynecol Pathol 2002; 21(4):375-382.

Bremnes RM, Veve R, Gabrielson E, Hirsch FR, Baron A, Bemis L et al. Highthroughput tissue microarray analysis used to evaluate biology and prognostic significance of the E-cadherin pathway in non-small-cell lung cancer. J Clin Oncol 2002; $20(10): 2417-2428$.

Bresalier RS, Mazurek N, Sternberg LR, Byrd JC, Yunker CK, Nangia-Makker P et al. Metastasis of human colon cancer is altered by modifying expression of the betagalactoside-binding protein galectin 3. Gastroenterology 1998; 115(2):287-296. Bringuier PP, Umbas R, Schaafsma HE, Karthaus HF, Debruyne FM, Schalken JA. Decreased E-cadherin immunoreactivity correlates with poor survival in patients with bladder tumors. Cancer Res 1993; 53(14):3241-3245. 
Bristow RE, Asrari F, Trimble EL, Montz FJ. Extended surgical staging for uterine papillary serous carcinoma: survival outcome of locoregional (Stage I-III) disease. Gynecol Oncol 2001; 81(2):279-286.

Broaddus RR, Lynch HT, Chen LM, Daniels MS, Conrad P, Munsell MF et al. Pathologic features of endometrial carcinoma associated with HNPCC: a comparison with sporadic endometrial carcinoma. Cancer 2006; 106(1):87-94.

Burton JL, Wells M. Recent advances in the histopathology and molecular pathology of carcinoma of the endometrium. Histopathology 1998; 33(4):297-303.

Busmanis I, Ho TH, Tan SB, Khoo KS. p53 and bcl-2 expression in invasive and pre-invasive uterine papillary serous carcinoma and atrophic endometrium. Ann Acad Med Singapore 2005; 34(7):421-425.

Buttery R, Monaghan H, Salter DM, Sethi T. Galectin-3: differential expression between small-cell and non-small-cell lung cancer. Histopathology 2004; 44(4):339344 .

Camp RL, Charette LA, Rimm DL. Validation of tissue microarray technology in breast carcinoma. Lab Invest 2000; 80(12):1943-1949.

Carcangiu ML, Chambers JT. Uterine papillary serous carcinoma: a study on 108 cases with emphasis on the prognostic significance of associated endometrioid carcinoma, absence of invasion, and concomitant ovarian carcinoma. Gynecol Oncol 1992; 47(3):298-305

Carcangiu ML, Chambers JT, Voynick IM, Pirro M, Schwartz PE. Immunohistochemical evaluation of estrogen and progesterone receptor content in 183 patients with endometrial carcinoma. Part I: Clinical and histologic correlations. Am J Clin Pathol 1990; 94(3):247-254.

Carcangiu ML, Tan LK, Chambers JT. Stage IA uterine serous carcinoma: a study of 13 cases. Am J Surg Pathol 1997; 21(12):1507-1514. 
Cassia R, Moreno-Bueno G, Rodriguez-Perales S, Hardisson D, Cigudosa JC, Palacios J. Cyclin E gene (CCNE) amplification and hCDC4 mutations in endometrial carcinoma. J Pathol 2003; 201(4):589-595.

Castelbaum AJ, Ying L, Somkuti SG, Sun J, Ilesanmi AO, Lessey BA. Characterization of integrin expression in a well differentiated endometrial adenocarcinoma cell line (Ishikawa). J Clin Endocrinol Metab 1997; 82(1):136-142. Castronovo V, van den Brule FA, Jackers P, Clausse N, Liu FT, Gillet C et al. Decreased expression of galectin-3 is associated with progression of human breast cancer. J Pathol 1996; 179(1):43-48.

Chambers AF, Groom AC, MacDonald IC. Dissemination and growth of cancer cells in metastatic sites. Nat Rev Cancer 2002; 2(8):563-572.

Chambers JT, Chambers SK, Kohorn EI, Carcangiu ML, Schwartz PE. Uterine papillary serous carcinoma treated with intraperitoneal cisplatin and intravenous doxorubicin and cyclophosphamide. Gynecol Oncol 1996; 60(3):438-442.

Chambers JT, Merino M, Kohorn EI, Peschel RE, Schwartz PE. Uterine papillary serous carcinoma. Obstet Gynecol 1987; 69(1):109-113.

Chandrasekaran S, Guo NH, Rodrigues RG, Kaiser J, Roberts DD. Pro-adhesive and chemotactic activities of thrombospondin-1 for breast carcinoma cells are mediated by alpha3 beta1 integrin and regulated by insulin-like growth factor- 1 and CD98. $J$ Biol Chem 1999; 274(16):11408-11416.

Chang C, Werb Z. The many faces of metalloproteases: cell growth, invasion, angiogenesis and metastasis. Trends Cell Biol 2001; 11(11):S37-S43. 
Chang WC, Sheu BC, Chen RC, Chow SN, Huang SC. Depressed host immunity in a case of metachronous primary uterine papillary serous carcinoma and non-Hodgkin's lymphoma. Int J Gynecol Cancer 2004; 14(5):1030-1032.

Chen GT, Getsios S, MacCalman CD. Progesterone regulates beta-catenin mRNA levels in human endometrial stromal cells in vitro. Endocrine 1998; 9(3):263-267.

Chen-Levy Z, Nourse J, Cleary ML. The bcl-2 candidate proto-oncogene product is a 24-kilodalton integral-membrane protein highly expressed in lymphoid cell lines and lymphomas carrying the $\mathrm{t}(14 ; 18)$ translocation. Mol Cell Biol 1989; 9(2):701-710.

Cherayil BJ, Weiner SJ, Pillai S. The Mac-2 antigen is a galactose-specific lectin that binds IgE. J Exp Med 1989; 170(6):1959-1972.

Christensen HN. Role of amino acid transport and countertransport in nutrition and metabolism. Physiol Rev 1990; 70(1):43-77.

Christman JE, Kapp DS, Hendrickson MR, Howes AE, Ballon SC. Therapeutic approaches to uterine papillary serous carcinoma: a preliminary report. Gynecol Oncol 1987; 26(2):228-235.

Christofori G, Semb H. The role of the cell-adhesion molecule E-cadherin as a tumour-suppressor gene. Trends Biochem Sci 1999; 24(2):73-76.

Christopherson WM, Alberhasky RC, Connelly PJ. Carcinoma of the endometrium. II. Papillary adenocarcinoma: a clinical pathological study, 46 cases. Am J Clin Pathol 1982; 77(5):534-540.

Cioppi F, Simi L, Luciani P, Petraglia F, Susini T, Cobellis L et al. Expression of uteroglobin and matrix metalloproteinase-9 genes in endometrial cancer: relationship to estrogen and progesterone receptor status. Oncol Rep 2004; 11(2):427-433. 
Cirisano FD, Jr., Robboy SJ, Dodge RK, Bentley RC, Krigman HR, Synan IS et al. Epidemiologic and surgicopathologic findings of papillary serous and clear cell endometrial cancers when compared to endometrioid carcinoma. Gynecol Oncol 1999; 74(3):385-394.

Cotran RS, Kumar V, Collins T. Neoplasia. In: Cotran RS, Kumar V, Collins T, editors. Pathologic Basis of Disease. London: WB Saunders, 2006: 260-327.

Crawford HC, Fingleton BM, Rudolph-Owen LA, Goss KJ, Rubinfeld B, Polakis P et al. The metalloproteinase matrilysin is a target of beta-catenin transactivation in intestinal tumors. Oncogene 1999; 18(18):2883-2891.

Creasman WT. New gynecologic cancer staging. Obstet Gynecol 1990; 75(2):287288.

Creasman WT, Odicino F, Maisonneuve P, Beller U, Benedet JL, Heintz AP et al. Carcinoma of the corpus uteri. Int J Gynaecol Obstet 2003; 83 Suppl 1:79-118.

Crescenzi E, Criniti V, Pianese M, Tecce MF, Palumbo G. Differential expression of antiapoptotic genes in human endometrial carcinoma: bcl-XL succeeds bcl-2 function in neoplastic cells. Gynecol Oncol 2000; 77(3):419-428.

Dagher SF, Wang JL, Patterson RJ. Identification of galectin-3 as a factor in premRNA splicing. Proc Natl Acad Sci U S A 1995; 92(4):1213-1217.

DeBernardo RL, Littell RD, Luo H, Duska LR, Oliva E, Kirley SD et al. Defining the extent of cables loss in endometrial cancer subtypes and its effectiveness as an inhibitor of cell proliferation in malignant endometrial cells in vitro and in vivo. Cancer Biol Ther 2005; 4(1):103-107.

Dembo AJ. The sequential multiple modality treatment of ovarian cancer. Radiother Oncol 1985; 3(3):187-192. 
Demopoulos RI, Mesia AF, Mittal K, Vamvakas E. Immunohistochemical comparison of uterine papillary serous and papillary endometrioid carcinoma: clues to pathogenesis. Int J Gynecol Pathol 1999; 18(3):233-237.

Derynck R, Akhurst RJ, Balmain A. TGF-beta signaling in tumor suppression and cancer progression. Nat Genet 2001; 29(2):117-129.

Deugnier MA, Faraldo MM, Rousselle P, Thiery JP, Glukhova MA. Cellextracellular matrix interactions and EGF are important regulators of the basal mammary epithelial cell phenotype. J Cell Sci 1999; 112 ( Pt 7):1035-1044.

Diamandis EP, Yousef GM. Human tissue Kallikreins: a family of new cancer biomarkers. Clin Chem 2002; 48:1198-1205.

Dong S, Hughes RC. Macrophage surface glycoproteins binding to galectin-3 (Mac2-antigen). Glycoconj J 1997; 14(2):267-274.

Dunton CJ, Balsara G, McFarland M, Hernandez E. Uterine papillary serous carcinoma: a review. Obstet Gynecol Surv 1991; 46(2):97-102.

Dvalishvili I, Charkviani L, Turashvili G, Burkadze G. The expression of cadherin e and clinical prognostic factors in uterine endometrioid adenocarcinoma. Georgian Med News 2005;(128):17-21.

Elit L, Hirte H. Current status and future innovations of hormonal agents, chemotherapy and investigational agents in endometrial cancer. Curr Opin Obstet Gynecol 2002; 14(1):67-73.

Endo K, Ueda T, Ueyama J, Ohta T, Terada T. Immunoreactive E-cadherin, alphacatenin, beta-catenin, and gamma-catenin proteins in hepatocellular carcinoma: relationships with tumor grade, clinicopathologic parameters, and patients' survival. Hum Pathol 2000; 31(5):558-565 
Esteban F, Ruiz-Cabello F, Concha A, Perez AM, Delgado M, Garrido F. Relationship of 4F2 antigen with local growth and metastatic potential of squamous cell carcinoma of the larynx. Cancer 1990; 66(7):1493-1498.

Estevez R, Camps M, Rojas AM, Testar X, Deves R, Hediger MA et al. The amino acid transport system $\mathrm{y}+\mathrm{L} / 4 \mathrm{~F} 2 \mathrm{hc}$ is a heteromultimeric complex. FASEB J 1998; 12(13):1319-1329.

Euscher ED, Malpica A, Deavers MT, Silva EG. Differential expression of WT-1 in serous carcinomas in the peritoneum with or without associated serous carcinoma in endometrial polyps. Am J Surg Pathol 2005; 29(8):1074-1078.

Fenczik CA, Sethi T, Ramos JW, Hughes PE, Ginsberg MH. Complementation of dominant suppression implicates CD98 in integrin activation. Nature 1997; 390(6655):81-85.

Fenczik CA, Zent R, Dellos M, Calderwood DA, Satriano J, Kelly C et al. Distinct domains of CD98he regulate integrins and amino acid transport. J Biol Chem 2001; 276(12):8746-8752.

Fernebro E, Dictor M, Bendahl PO, Ferno M, Nilbert M. Evaluation of the tissue microarray technique for immunohistochemical analysis in rectal cancer. Arch Pathol Lab Med 2002; 126(6):702-705.

Frank AH, Tseng PC, Haffty BG, Papadopoulos DP, Kacinski BM, Dowling SW et al. Adjuvant whole-abdominal radiation therapy in uterine papillary serous carcinoma. Cancer 1991; 68(7):1516-1519.

Fujimoto J, Ichigo S, Hori M, Morishita S, Tamaya T. Progestins and danazol effect on cell-to-cell adhesion, and E-cadherin and alpha- and beta-catenin mRNA expressions. J Steroid Biochem Mol Biol 1996; 5-6:275-282. 
Fujimoto J, Sakaguchi H, Hirose R, Tamaya T. Significance of sex steroids in roles of cadherin subfamily and its related proteins in the uterine endometrium and placenta. Horm Res 1998; 50 Suppl 2:30-36.

Fukasawa Y, Segawa H, Kim JY, Chairoungdua A, Kim DK, Matsuo H et al. Identification and characterization of $\mathrm{a} \mathrm{Na}(+)$-independent neutral amino acid transporter that associates with the 4F2 heavy chain and exhibits substrate selectivity for small neutral D- and L-amino acids. J Biol Chem 2000; 275(13):9690-9698.

Gallion HH, van NJ, Jr., Powell DF, Donaldson ES, Higgins RV, Kryscio RJ et al. Stage I serous papillary carcinoma of the endometrium. Cancer 1989; 63(11):22242228.

Gamallo C, Moreno-Bueno G, Sarrio D, Calero F, Hardisson D, Palacios J. The prognostic significance of $\mathrm{P}$-cadherin in infiltrating ductal breast carcinoma. Mod Pathol 2001; 14(7):650-654.

Gehrig PA, Groben PA, Fowler WC, Jr., Walton LA, Van Le L. Noninvasive papillary serous carcinoma of the endometrium. Obstet Gynecol 2001; 97(1):153157.

Gehrig PA, Morris DE, Van Le L. Uterine serous carcinoma: a comparison of therapy for advanced-stage disease. Int J Gynecol Cancer 2004; 14(3):515-520.

Gehrig PA, Van Le L, Fowler WC, Jr. The role of omentectomy during the surgical staging of uterine serous carcinoma. Int J Gynecol Cancer 2003; 13(2):212-215.

Geisler JP, Geisler HE, Melton ME, Wiemann MC. What staging surgery should be performed on patients with uterine papillary serous carcinoma? Gynecol Oncol 1999; $74(3): 465-467$ 
Geisler JP, Sorosky JI, Duong HL, Buekers TE, Geisler MJ, Sood AK et al. Papillary serous carcinoma of the uterus: increased risk of subsequent or concurrent development of breast carcinoma. Gynecol Oncol 2001; 83(3):501-503.

Gibbons S, Martinez A, Schray M, Podratz K, Stanhope R, Garton G et al. Adjuvant whole abdominopelvic irradiation for high risk endometrial carcinoma. Int J Radiat Oncol Biol Phys 1991; 21(4):1019-1025.

Gillenwater A, Xu XC, el Naggar AK, Clayman GL, Lotan R. Expression of galectins in head and neck squamous cell carcinoma. Head Neck 1996; 18(5):422432.

Gillett CE, Springall RJ, Barnes DM, Hanby AM. Multiple tissue core arrays in histopathology research: a validation study. J Pathol 2000; 192(4):549-553.

Gitsch G, Friedlander ML, Wain GV, Hacker NF. Uterine papillary serous carcinoma. A clinical study. Cancer 1995; 75(9):2239-2243.

Glinsky VV, Glinsky GV, Glinskii OV, Huxley VH, Turk JR, Mossine VV et al. Intravascular metastatic cancer cell homotypic aggregation at the sites of primary attachment to the endothelium. Cancer Res 2003; 63(13):3805-3811.

Goff BA, Kato D, Schmidt RA, Ek M, Ferry JA, Muntz HG et al. Uterine papillary serous carcinoma: patterns of metastatic spread. Gynecol Oncol 1994; 54(3):264268.

Gomaa W, Ke Y, Fujii H, Helliwell T. Tissue microarray of head and neck squamous carcinoma: validation of the methodology for the study of cutaneous fatty acidbinding nrotein. vascular endothelial growth factor. involucrin and Ki-67. Virchows Arch 2005; 447(4):701-709. 
Gore M, Oza A, Rustin G, Malfetano J, Calvert H, Clarke-Pearson D et al. A randomised trial of oral versus intravenous topotecan in patients with relapsed epithelial ovarian cancer. Eur J Cancer 2002; 38(1):57-63.

Goshen R, Chu W, Elit L, Pal T, Hakimi J, Ackerman I et al. Is uterine papillary serous adenocarcinoma a manifestation of the hereditary breast-ovarian cancer syndrome? Gynecol Oncol 2000; 79(3):477-481.

Goyal J, Smith KM, Cowan JM, Wazer DE, Lee SW, Band V. The role for NES1 serine protease as a novel tumour suppressor. Cancer Res 1998; 58:4782-4786.

Guilford P, Hopkins J, Harraway J, McLeod M, McLeod N, Harawira P et al. Ecadherin germline mutations in familial gastric cancer. Nature 1998; 392(6674):402405 .

Gulmann C, Butler D, Kay E, Grace A, Leader M. Biopsy of a biopsy: validation of immunoprofiling in gastric cancer biopsy tissue microarrays. Histopathology 2003; 42(1):70-76.

Han AC, Edelson MI, Peralta SA, Knudsen KA, Lifschitz-Mercer B, Czernobilsky B et al. Cadherin expression in glandular tumors of the cervix. Cancer 2000; 89(10):2053-2058.

Hara K, Kudoh H, Enomoto T, Hashimoto Y, Masuko T. Malignant transformation of NIH3T3 cells by overexpression of early lymphocyte activation antigen CD98 207. Biochem Biophys Res Commun 1999; 262(3):720-725.

Hara K, Kudoh H, Enomoto T, Hashimoto Y, Masuko T. Enhanced tumorigenicity caused by truncation of the extracellular domain of GP125/CD98 heavy chain 161. Oncogene $2000 ; 19(54): 6209-6215$.

Hasegawa S, Koshikawa N, Momiyama N, Moriyama K, lchikawa $Y$, Ishikawa $T$ et al. Matrilysin-speciñc antisense oligonucieotide inhibits iiver metastasis of numan colon cancer ceils in a nude mouse model. int 3 Cancer $1998 ; 76(6): 812-816$. 
Haynes BF, Hemler ME, Mann DL, Eisenbarth GS, Shelhamer J, Mostowski HS et al. Characterization of a monoclonal antibody (4F2) that binds to human monocytes and to a subset of activated lymphocytes. J Immunol 1981; 126(4):1409-1414.

Hemler ME, Strominger JL. Characterization of antigen recognized by the monoclonal antibody (4F2): different molecular forms on human $\mathrm{T}$ and $\mathrm{B}$ lymphoblastoid cell lines. J Immunol 1982; 129(2):623-628.

Hendrickson M, Ross J, Eifel P, Martinez A, Kempson R. Uterine papillary serous carcinoma: a highly malignant form of endometrial adenocarcinoma. Am J Surg Pathol 1982; 6(2):93-108.

Hendrickson M, Ross J, Eifel PJ, Cox RS, Martinez A, Kempson R.

Adenocarcinoma of the endometrium: analysis of 256 cases with carcinoma limited to the uterine corpus. Pathology review and analysis of prognostic variables. Gynecol Oncol 1982; 13(3):373-392.

Hendrickson MR, Ross JC, Kempson RL. Toward the development of morphologic criteria for well-differentiated adenocarcinoma of the endometrium. Am J Surg Pathol 1983; 7(8):819-838.

Herzog TJ, Kowalski LD, Liu H, Arlt M, Swisher E. Evaluation of a region on chromosome $1 \mathrm{p}$ in ovarian serous carcinoma that is frequently deleted in uterine papillary serous carcinoma. Gynecol Oncol 2001; 82(1):139-142.

Hicks ML, Phillips JL, Parham G, Andrews N, Jones WB, Shingleton HM et al. The National Cancer Data Base report on endometrial carcinoma in African-American women. Cancer 1998; 83(12):2629-2637.

Hinck L, Nathke IS, Papkoff J, Nelson WJ. Dynamics of cadherin/catenin complex formation: novel protein interactions and pathwavs of complex assemblv. J Cell Biol $1994 ; 125(6): 1327-1340$. 
Hirano T. Interleukin 6 and its receptor: ten years later. Int Rev Immunol 1998; $16(3-4): 249-284$.

Hoek K, Rimm DL, Williams KR, Zhao H, Ariyan S, Lin A et al. Expression profiling reveals novel pathways in the transformation of melanocytes to melanomas. Cancer Res 2004; 64(15):5270-5282.

Holcomb K, Delatorre R, Pedemonte B, McLeod C, Anderson L, Chambers J. Ecadherin expression in endometrioid, papillary serous, and clear cell carcinoma of the endometrium. Obstet Gynecol 2002; 100(6):1290-1295.

Honjo Y, Inohara H, Akahani S, Yoshii T, Takenaka Y, Yoshida J et al. Expression of cytoplasmic galectin-3 as a prognostic marker in tongue carcinoma. Clin Cancer Res 2000; 6(12):4635-4640.

Hood JD, Cheresh DA. Role of integrins in cell invasion and migration. Nat Rev Cancer 2002; 2(2):91-100.

Hoos A, Cordon-Cardo C. Tissue microarray profiling of cancer specimens and cell lines: opportunities and limitations. Lab Invest 2001; 81(10):1331-1338.

Hoos A, Urist MJ, Stojadinovic A, Mastorides S, Dudas ME, Leung DH et al. Validation of tissue microarrays for immunohistochemical profiling of cancer specimens using the example of human fibroblastic tumors. Am J Pathol 2001; $158(4): 1245-1251$.

Hornreich G, Beller U, Lavie O, Renbaum P, Cohen Y, Levy-Lahad E. Is uterine serous papillary carcinoma a BRCA1-related disease? Case report and review of the literature. Gynecol Oncol 1999; 75(2):300-304.

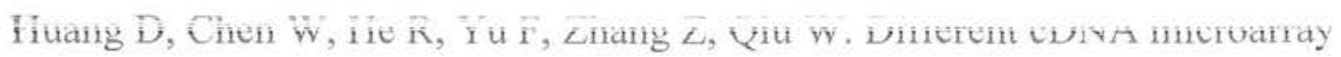
patterns of gene expression refiecting changes during metastatic progression in adenoid cystic carcinoma. World J Surg Uncol 2003; 1(1):28. 
Hughes RC. Mac-2: a versatile galactose-binding protein of mammalian tissues. Glycobiology 1994; 4(1):5-12.

Huh WK, Powell M, Leath CA, III, Straughn JM, Jr., Cohn DE, Gold MA et al. Uterine papillary serous carcinoma: comparisons of outcomes in surgical Stage I patients with and without adjuvant therapy. Gynecol Oncol 2003; 91(3):470-475.

Hui P, Kelly M, O'malley DM, Tavassoli F, Schwartz PE. Minimal uterine serous carcinoma: a clinicopathological study of 40 cases. Mod Pathol 2005; 18(1):75-82.

Idrees MT, Schlosshauer P, Li G, Burstein DE, . GLUT1 and p63 expression in endometrial intraepithelial and uterine serous papillary carcinoma. Histopathology 49[1], 75-81. 2006.

Inohara H, Akahani S, Koths K, Raz A. Interactions between galectin-3 and Mac-2binding protein mediate cell-cell adhesion. Cancer Res 1996; 56(19):4530-4534.

Inohara H, Raz A. Functional evidence that cell surface galectin-3 mediates homotypic cell adhesion. Cancer Res 1995; 55(15):3267-3271.

Inoue Y, Abe K, Obata K, Yoshioka T, Ohmura G, Doh K et al. Immunohistochemical studies on matrix metalloproteinase-9 (MMP-9) and type-IV collagen in endometrial carcinoma. J Obstet Gynaecol Res 1997; 23(2):139-145.

Itoh S, Itoh F, Goumans MJ, ten Dijke P. Signaling of transforming growth factorbeta family members through Smad proteins. Eur J Biochem 2000; 267(24):69546967.

Iurlaro M, Loverro G, Vacca A, Cormio G, Ribatti D, Minischetti M et al. Angiogenesis extent and expression of matrix metalloproteinase- 2 and -9 correlate with upgrading and myometrial invasion in endometrial carcinoma. Eur J Clin Invest 1999; 29(9):793-801. 
Jeffrey JF, Krepart GV, Lotocki RJ. Papillary serous adenocarcinoma of the endometrium. Obstet Gynecol 1986; 67(5):670-674.

Jiang Y, Goldberg ID, Shi YE. Complex roles of tissue inhibitors of metalloproteinases in cancer. Oncogene 2002; 21(14):2245-2252.

Jin LH, Shao QJ, Luo W, Ye ZY, Li Q, Lin SC. Detection of point mutations of the Axin1 gene in colorectal cancers. Int J Cancer 2003; 107(5):696-699.

John A, Tuszynski G. The role of matrix metalloproteinases in tumor angiogenesis and tumor metastasis. Pathol Oncol Res 2001; 7(1):14-23.

Jou TS, Stewart DB, Stappert J, Nelson WJ, Marrs JA. Genetic and biochemical dissection of protein linkages in the cadherin-catenin complex. Proc Natl Acad Sci U S A 1995; 92(11):5067-5071.

Kakugawa K, Hattori M, Beauchemin N, Minato N. Activation of CEA-CAM-1mediated cell adhesion via CD98: involvement of PKCdelta. FEBS Lett 2003; 552(23):184-188.

Kallioniemi OP, Wagner U, Kononen J, Sauter G. Tissue microarray technology for high-throughput molecular profiling of cancer. Hum Mol Genet 2001; 10(7):657662.

Kanai Y, Fukasawa Y, Cha SH, Segawa H, Chairoungdua A, Kim DK et al. Transport properties of a system $\mathrm{y}+\mathrm{L}$ neutral and basic amino acid transporter. Insights into the mechanisms of substrate recognition. J Biol Chem 2000; 275(27):20787-20793.

Kanai Y, Segawa H, Miyamoto K, Uchino H, Takeda E, Endou H. Expression cloning and characterization of a transporter for large neutral amino acids activated by the heavy chain of 4F2 antigen (CD98). J Biol Chem 1998; 273(37):23629-23632. 
Kannagi R. Carbohydrate-mediated cell adhesion involved in hematogenous metastasis of cancer. Glycoconj J 1997; 14(5):577-584.

Kariola R, Abdel-Rahman WM, Ollikainen M, Butzow R, Peltomaki P, Nystrom M. APC and beta-catenin protein expression patterns in HNPCC-related endometrial and colorectal cancers. Fam Cancer 2005; 4(2):187-190.

Kato DT, Ferry JA, Goodman A, Sullinger J, Scully RE, Goff BA et al. Uterine papillary serous carcinoma (UPSC): a clinicopathologic study of 30 cases. Gynecol Oncol 1995; 59(3):384-389.

Kelly MG, O'malley DM, Hui P, McAlpine J, Yu H, Rutherford TJ et al. Improved survival in surgical stage I patients with uterine papillary serous carcinoma (UPSC) treated with adjuvant platinum-based chemotherapy. Gynecol Oncol 2005; $98(3): 353-359$.

Kerr JF, Wyllie AH, Currie AR. Apoptosis: a basic biological phenomenon with wide-ranging implications in tissue kinetics. Br J Cancer 1972; 26(4):239-257.

Kihana T, Yano N, Murao S, Iketani H, Hamada K, Yano J et al. Allelic loss of chromosome $16 \mathrm{q}$ in endometrial cancer: correlation with poor prognosis of patients and less differentiated histology. Jpn J Cancer Res 1996; 87(11):1184-1190.

Kim dK, Ahn SG, Park JC, Kanai Y, Endou H, Yoon JH. Expression of L-type amino acid transporter 1 (LAT1) and 4F2 heavy chain (4F2hc) in oral squamous cell carcinoma and its precusor lesions. Anticancer Res 2004; 24(3a):1671-1675.

Kim dK, Kanai Y, Choi HW, Tangtrongsup S, Chairoungdua A, Babu E et al. Characterization of the system L amino acid transporter in T24 human bladder carcinoma cells. Biochim Biophys Acta 2002; 1565(1):112-121. 
King SA, Adas AA, LiVolsi VA, Takahashi H, Behbakht K, McGovern P et al. Expression and mutation analysis of the $\mathrm{p} 53$ gene in uterine papillary serous carcinoma. Cancer 1995; 75(11):2700-2705.

Kishida S, Yamamoto H, Ikeda S, Kishida M, Sakamoto I, Koyama S et al. Axin, a negative regulator of the wnt signaling pathway, directly interacts with adenomatous polyposis coli and regulates the stabilization of beta-catenin. J Biol Chem 1998; 273(18):10823-10826.

Kononen J, Bubendorf L, Kallioniemi A, Barlund M, Schraml P, Leighton S et al. Tissue microarrays for high-throughput molecular profiling of tumor specimens. Nat Med 1998; 4(7):844-847.

Koppert LB, van der Velden AW, van de WM, Abbou M, van den Ouweland AM, Tilanus HW et al. Frequent loss of the AXIN1 locus but absence of AXIN1 gene mutations in adenocarcinomas of the gastro-oesophageal junction with nuclear betacatenin expression. Br J Cancer 2004; 90(4):892-899.

Koshiyama M, Konishi I, Mandai M, Komatsu T, Yamamoto S, Nanbu K et al. Immunohistochemical analysis of $\mathrm{p} 53$ protein and $72 \mathrm{kDa}$ heat shock protein (HSP72) expression in ovarian carcinomas. Correlation with clinicopathology and sex steroid receptor status. Virchows Arch 1995; 425(6):603-609.

Kovalev S, Marchenko ND, Gugliotta BG, Chalas E, Chumas J, Moll UM. Loss of p53 function in uterine papillary serous carcinoma. Hum Pathol 1998; 29(6):613619.

Kowalczyk AP, Reynolds AB. Protecting your tail: regulation of cadherin degradation by p120-catenin. Curr Opin Cell Biol 2004; 16(5):522-527. Kugler A. Matrix metalloproteinases and their inhibitors. Anticancer Res 1999; 19(2C):1589-1592. 
Lauchlan SC. Tubal (serous) carcinoma of the endometrium. Arch Pathol Lab Med 1981; 105(11):615-618.

Lavie O, Hornreich G, Ben Arie A, Renbaum P, Levy-Lahad E, Beller U. BRCA1 germline mutations in women with uterine serous papillary carcinoma. Obstet Gynecol 2000; 96(1):28-32.

Lavie O, Hornreich G, Ben Arie A, Rennert G, Cohen Y, Keidar R et al. BRCA germline mutations in Jewish women with uterine serous papillary carcinoma. Gynecol Oncol 2004; 92(2):521-524.

Lax SF. Molecular genetic pathways in various types of endometrial carcinoma: from a phenotypical to a molecular-based classification. Virchows Arch 2004; 444(3):213-223.

Le Marer N, Hughes RC. Effects of the carbohydrate-binding protein galectin-3 on the invasiveness of human breast carcinoma cells. J Cell Physiol 1996; 168(1):51-58.

Leblanc M, Poncelet C, Soriano D, Walker-Combrouze F, Madelenat P, Scoazec JY et al. Alteration of CD44 and cadherins expression: possible association with augmented aggressiveness and invasiveness of endometrial carcinoma. Virchows Arch 2001; 438(1):78-85.

Lee KR, Belinson JL. Recurrence in noninvasive endometrial carcinoma. Relationship to uterine papillary serous carcinoma. Am J Surg Pathol 1991; 15(10):965-973.

Lee YC, Wu CT, Chen CS, Hsu HH, Chang YL. The significance of E-cadherin and alpha-, beta-, and gamma-catenin expression in surgically treated non-small cell lung cancers of $3 \mathrm{~cm}$ or less in size. J Thorac Cardiovasc Surg 2002; 123(3):502-507. 
Lemaitre G, Gonnet F, Vaigot P, Gidrol X, Martin MT, Tortajada J et al. CD98, a novel marker of transient amplifying human keratinocytes. Proteomics 2005; 5(14):3637-3645.

Lessey BA, Albelda S, Buck CA, Castelbaum AJ, Yeh I, Kohler M et al. Distribution of integrin cell adhesion molecules in endometrial cancer. Am J Pathol 1995; 146(3):717-726.

Levenback C, Burke TW, Silva E, Morris M, Gershenson DM, Kavanagh JJ et al. Uterine papillary serous carcinoma (UPSC) treated with cisplatin, doxorubicin, and cyclophosphamide (PAC). Gynecol Oncol 1992; 46(3):317-321.

Leversha MA, Fielding P, Watson S, Gosney JR, Field JK. Expression of p53, pRB, and p16 in lung tumours: a validation study on tissue microarrays. J Pathol 2003; 200(5):610-619.

Lim P, Al Kushi A, Gilks B, Wong F, Aquino-Parsons C. Early stage uterine papillary serous carcinoma of the endometrium: effect of adjuvant whole abdominal radiotherapy and pathologic parameters on outcome. Cancer 2001; 91(4):752-757.

Lim SC, Lee MS. Significance of E-cadherin/beta-catenin complex and cyclin D1 in breast cancer. Oncol Rep 2002; 9(5):915-928.

Liokumovich P, Goldberg I, Davidson B, Gotlieb WH, Zahavi T, Ben Baruch G et al. Expression of metalloproteinases endometrial stromal sarcoma: immunohistochemical study using image analysis. J Clin Pathol 1999; 52(3): 198202.

Liotta LA. Tumor invasion and metastases--role of the extracellular matrix: Rhoads Memorial Award lecture. Cancer Res 1986: 46(1):1-7.

Liu FS, Chen JT, Hsieh YT, Ho ES, Hung MJ, Lu CH et al. Loss of Smad4 protein expression occurs infrequently in endometrial carcinomas. Int $\mathrm{J}$ Gynecol Pathol $22[4], 347-352.1-10-2003$. 
Liu C, Li Y, Semenov M, Han C, Baeg GH, Tan Y et al. Control of beta-catenin phosphorylation/degradation by a dual-kinase mechanism. Cell 2002; 108(6):837847.

Liu W, Dong X, Mai M, Seelan RS, Taniguchi K, Krishnadath KK et al. Mutations in AXIN2 cause colorectal cancer with defective mismatch repair by activating betacatenin/TCF signalling. Nat Genet 2000; 26(2):146-147.

Lloyd RV, Erickson LA, Jin L, Kulig E, Qian X, Cheville JC et al. p27kip1: a multifunctional cyclin-dependent kinase inhibitor with prognostic significance in human cancers. Am J Pathol 1999; 154(2):313-323.

Lo ML, Campisi G, Farina A, Rubini C, Pannone G, Serpico R et al. P-cadherin expression and survival rate in oral squamous cell carcinoma: an immunohistochemical study. BMC Cancer 2005; 5:63.

Lotz MM, Andrews CW, Jr., Korzelius CA, Lee EC, Steele GD, Jr., Clarke A et al. Decreased expression of Mac-2 (carbohydrate binding protein 35) and loss of its nuclear localization are associated with the neoplastic progression of colon carcinoma. Proc Natl Acad Sci U S A 1993; 90(8):3466-3470.

Lu QL, Abel P, Foster CS, Lalani EN. bcl-2: role in epithelial differentiation and oncogenesis. Hum Pathol 1996; 27(2):102-110.

Lumadue JA, Glick AB, Ruddle FH. Cloning, sequence analysis, and expression of the large subunit of the human lymphocyte activation antigen 4F2. Proc Natl Acad Sci U S A 1987; 84(24):9204-9208.

Macwhinnie N, Monaghan H. The use of P53, PTEN, and C-erbB-2 to differentiate uterine serous nanillary carcinoma from endometrioid endometrial carcinoma. Int $\mathrm{J}$ Gunesol Cancer $2004 \cdot 14(5) \cdot 938-946$ 
Magriples U, Naftolin F, Schwartz PE, Carcangiu ML. High-grade endometrial carcinoma in tamoxifen-treated breast cancer patients. J Clin Oncol 1993; 11(3):485490.

Mallipeddi P, Kapp DS, Teng NN. Long-term survival with adjuvant whole abdominopelvic irradiation for uterine papillary serous carcinoma. Cancer 1993; 71(10):3076-3081.

Mannion BA, Kolesnikova TV, Lin SH, Wang S, Thompson NL, Hemler ME. The light chain of CD98 is identified as E16/TA1 protein. J Biol Chem 1998; 273(50):33127-33129.

Martelli M, Campana A, Bischof P. Secretion of matrix metalloproteinases by human endometrial cells in vitro. J Reprod Fertil 1993; 98(1):67-76.

Massague J. How cells read TGF-beta signals. Nat Rev Mol Cell Biol 2000; 1(3):169-178.

Massague J, Blain SW, Lo RS. TGFbeta signaling in growth control, cancer, and heritable disorders. Cell 2000; 103(2):295-309.

Mastroberardino L, Spindler B, Pfeiffer R, Skelly PJ, Loffing J, Shoemaker CB et al. Amino-acid transport by heterodimers of $4 \mathrm{~F} 2 \mathrm{hc} / \mathrm{CD} 98$ and members of a permease family. Nature 1998; 395(6699):288-291.

Matarrese P, Fusco O, Tinari N, Natoli C, Liu FT, Semeraro ML et al. Galectin-3 overexpression protects from apoptosis by improving cell adhesion properties. Int $\mathrm{J}$ Cancer 2000; 85(4):545-554.

Matrisian LM. The matrix-degrading metalloproteinases. Bioessays 1992; 14(7):455463.

Matrisian LM. Matrix metalloproteinase gene expression. Ann N Y Acad Sci 1994; $732: 42-50$. 
Matsuzaki F, Mege RM, Jaffe SH, Friedlander DR, Gallin WJ, Goldberg JI et al. cDNAs of cell adhesion molecules of different specificity induce changes in cell shape and border formation in cultured S180 cells. J Cell Biol 1990; 110(4):12391252.

McGuire WP, Blessing JA, Bookman MA, Lentz SS, Dunton CJ. Topotecan has substantial antitumor activity as first-line salvage therapy in platinum-sensitive epithelial ovarian carcinoma: A Gynecologic Oncology Group Study. J Clin Oncol 2000; 18(5):1062-1067.

Mehta N, Yamada SD, Rotmensch J, Mundt AJ. Outcome and pattern of failure in pathologic stage I-II papillary serous carcinoma of the endometrium: implications for adjuvant radiation therapy. Int J Radiat Oncol Biol Phys 2003; 57(4):1004-1009.

Meromsky L, Lotan R, Raz A. Implications of endogenous tumor cell surface lectins as mediators of cellular interactions and lung colonization. Cancer Res 1986: $46(10): 5270-5275$.

Milde-Langosch K, Bamberger AM, Goemann C, Rossing E, Rieck G, Kelp B et al. Expression of cell-cvcle regulatorv proteins in endoemtrial carcinomas: correlations with hormone receptor status and clinicopathologic parameters. J.Cancer Res.Clin.Oncol. 127Г91. 537-544. 2001.

Misugi F, Sumi T, Okamoto E, Nobeyama H, Hattori K, Yoshida H et al. Expression of matrix metalloproteinases and tissue inhibitors of metalloproteinase in uterine endometrial carcinoma and a correlation between expression of matrix metalloproteinase-7 and prognosis. Int J Mol Med 2005: 16(4):541-546.

Miyamoto S, Baba H, Kuroda S, Kaibuchi K, Fukuda T, Maehara Y et al. Changes in E-cadherin associated with cvtoplasmic molecules in well and poorlv differentiated endometrial cancer $\mathrm{Br}$ I Cancer $2000 \cdot 83191.1168-1175$ 
Miyazono K, ten Dijke P, Heldin CH. TGF-beta signaling by Smad proteins. Adv Immunol 2000; 75:115-157.

Moll UM, Chalas E, Auguste M, Meaney D, Chumas J. Uterine papillary serous carcinoma evolves via a p53-driven pathway. Hum Pathol 1996; 27(12):1295-1300.

Moreno-Bueno G, Hardisson D, Prat J, Matias-Guiu J, Palacios J. Re: Scholten et al. Nuclear beta-catenin is a molecular feature of type I endometrial carcinoma. J Pathol $2003 ; 201: 460-465$

1. J Pathol 2004; 202(4):511-512.

Moreno-Bueno G, Hardisson D, Sanchez C, Sarrio D, Cassia K, Garcia-Kostan G et al. Abnormalities of the APC/beta-catenin pathway in endometrial cancer. Oncogene 2002; 21(52):7981-7990.

Moreno-Bueno G, Hardisson D, Sarrio D, Sanchez C, Cassia R, Prat J et al. Abnormalities of E- and P-cadherin and catenin (beta-, gamma-catenin, and p120ctn) expression in endometrial cancer and endometrial atypical hyperplasia. J Pathol $2003 ; 199(4): 471-478$.

Moreno-Bueno G, Rodriguez-Perales S, Sanchez-Estevez C, Marcos R, Hardisson D, Cigudosa JC et al. Molecular alterations associated with cyclin D1 overexpression in endometrial cancer. Int J Cancer 2004; 110(2):194-200.

Morin PJ, Sparks AB, Korinek V, Barker N, Clevers H, Vogelstein B et al. Activation of beta-catenin-Tcf signaling in colon cancer by mutations in beta-catenin or APC. Science 1997; 275(5307):1787-1790.

Moscatello DK, Holgado-Madruga M, Godwin AK, Ramirez G, Gunn G, Zoltick PW et al. Frequent expression of a mutant epidermal growth factor receptor in multiple human tumors. Cancer Res 1995: 55(23):5536-5539. 
Moser PL, Kieback DG, Hefler L, Tempfer C, Neunteufel W, Gitsch G.

Immunohistochemical detection of matrix metalloproteinases (MMP) 1 and 2, and tissue inhibitor of metalloproteinase 2 (TIMP 2) in stage IB cervical cancer. Anticancer Res 1999; 19(5C):4391-4393.

Mousses S, Bubendorf L, Wagner U, Hostetter G, Kononen J, Cornelison R et al. Clinical validation of candidate genes associated with prostate cancer progression in the CWR22 model system using tissue microarrays. Cancer Res 2002; 62(5):12561260.

Munemitsu S, Albert I, Souza B, Rubinfeld B, Polakis P. Regulation of intracellular beta-catenin levels by the adenomatous polyposis coli (APC) tumor-suppressor protein. Proc Natl Acad Sci U S A 1995; 92(7):3046-3050.

Mylona E, Kapranou A, Mavrommatis J, Markaki S, Keramopoulos A, Nakopoulou L. The multifunctional role of the immunohistochemical expression of MMP-7 in invasive breast cancer. APMIS 2005; 113(4):246-255.

Nakamura E, Sato M, Yang H, Miyagawa F, Harasaki M, Tomita K et al. 4F2 (CD98) heavy chain is associated covalently with an amino acid transporter and controls intracellular trafficking and membrane topology of 4F2 heterodimer. J Biol Chem 1999; 274(5):3009-3016.

Nakashima J, Tachibana M, Horiguchi Y, Oya M, Ohigashi T, Asakura H et al. Serum interleukin 6 as a prognostic factor in patients with prostate cancer. Clin Cancer Res 2000; 6(7):2702-2706.

Nangia-Makker P, Honjo Y, Sarvis R, Akahani S, Hogan V, Pienta KJ et al. Galectin-3 induces endothelial cell morphogenesis and angiogenesis. Am J Pathol 2000: $156(3): 899-909$ 
Nathke IS, Hinck L, Swedlow JR, Papkoff J, Nelson WJ. Defining interactions and distributions of cadherin and catenin complexes in polarized epithelial cells. J Cell Biol 1994; 125(6):1341-1352.

Nei H, Saito T, Yamasaki H, Mizumoto H, Ito E, Kudo R. Nuclear localization of beta-catenin in normal and carcinogenic endometrium. Mol Carcinog 1999; 25(3):207-218.

Nelson AR, Fingleton B, Rothenberg ML, Matrisian LM. Matrix metalloproteinases: biologic activity and clinical implications. J Clin Oncol 2000; 18(5):1135-1149.

Newell KJ, Witty JP, Rodgers WH, Matrisian LM. Expression and localization of matrix-degrading metalloproteinases during colorectal tumorigenesis. Mol Carcinog 1994; 10(4):199-206.

Nicklin JL, Copeland LJ. Endometrial papillary serous carcinoma: patterns of spread and treatment. Clin Obstet Gynecol 1996; 39(3):686-695.

Niessen CM, Gumbiner BM. Cadherin-mediated cell sorting not determined by binding or adhesion specificity. J Cell Biol 2002; 156(2):389-399.

Nose A, Nagafuchi A, Takeichi M. Expressed recombinant cadherins mediate cell sorting in model systems. Cell 1988; 54(7):993-1001.

Ochieng J, Warfield P, Green-Jarvis B, Fentie I. Galectin-3 regulates the adhesive interaction between breast carcinoma cells and elastin. J Cell Biochem 1999; $75(3): 505-514$.

Olson MW, Toth M, Gervasi DC, Sado Y, Ninomiya Y, Fridman R. High affinity binding of latent matrix metalloproteinase- 9 to the alpha2(IV) chain of collagen IV. J Biol Chem 1998: 273(17):10672-10681. 
Oltvai ZN, Milliman CL, Korsmeyer SJ. Bcl-2 heterodimerizes in vivo with a conserved homolog, Bax, that accelerates programmed cell death. Cell 1993; 74(4):609-619.

Openo KP, Kadrofske MM, Patterson RJ, Wang JL. Galectin-3 expression and subcellular localization in senescent human fibroblasts. Exp Cell Res 2000; 255(2):278-290.

Orr FW, Wang HH, Lafrenie RM, Scherbarth S, Nance DM. Interactions between cancer cells and the endothelium in metastasis. J Pathol 2000; 190(3):310-329.

Palacios J, Benito N, Pizarro A, Limeres MA, Suarez A, Cano A et al. Relationship between ERBB2 and E-cadherin expression in human breast cancer. Virchows Arch $1995 ; 427(3): 259-263$.

Palacios J, Benito N, Pizarro A, Suarez A, Espada J, Cano A et al. Anomalous expression of $\mathrm{P}$-cadherin in breast carcinoma. Correlation with E-cadherin expression and pathological features. Am J Pathol 1995; 146(3):605-612.

Palacios J, Catasus L, Moreno-Bueno G, Matias-Guiu X, Prat J, Gamallo C. Betaand gamma-catenin expression in endometrial carcinoma. Relationship with clinicopathological features and microsatellite instability. Virchows Arch 2001; 438(5):464-469.

Pantel K, Passlick B, Vogt J, Stosiek P, Angstwurm M, Seen-Hibler R et al. Reduced expression of plakoglobin indicates an unfavorable prognosis in subsets of patients with non-small-cell lung cancer. J Clin Oncol 1998; 16(4):1407-1413.

Park DW, Ryu HS, Choi DS, Park YH, Chang KH, Min CK. Localization of matrix metalloproteinases on endometrial cancer cell invasion in vitro. Gynecol Oncol 2001; $82(3): 442-449$. 
Parks WC. Matrix metalloproteinases in repair. Wound Repair Regen 1999; $7(6): 423-432$.

Perl AK, Wilgenbus P, Dahl U, Semb H, Christofori G. A causal role for E-cadherin in the transition from adenoma to carcinoma. Nature 1998; 392(6672):190-193.

Pfeiffer R, Rossier G, Spindler B, Meier C, Kuhn L, Verrey F. Amino acid transport of $\mathrm{y}+\mathrm{L}$-type by heterodimers of 4F2hc/CD98 and members of the glycoproteinassociated amino acid transporter family. EMBO J 1999; 18(1):49-57.

Pfeiffer R, Spindler B, Loffing J, Skelly PJ, Shoemaker CB, Verrey F. Functional heterodimeric amino acid transporters lacking cysteine residues involved in disulfide bond. FEBS Lett 1998; 439(1-2):157-162.

Piestrzeniewicz-Ulanska D, Brys M, Semczuk A, Rechberger T, Jakowicki JA, Krajewska WM. TGF-beta signaling is disrupted in endometrioid-type endometrial carcinomas. Gynecol Oncol 2004; 95(1):173-180.

Pijnenborg JM, Kisters N, van Engeland M, Dunselman GA, de Haan J, de Goeij AF et al. APC, beta-catenin, and E-cadherin and the development of recurrent endometrial carcinoma. Int J Gynecol Cancer 2004; 14(5):947-956.

Pineda M, Fernandez E, Torrents D, Estevez R, Lopez C, Camps M et al. Identification of a membrane protein. LAT-2. that Co-expresses with 4F2 heavv chain, an L-type amino acid transport activity with broad specificity for small and large zwitterionic amino acids. J Biol Chem 1999: 274(28):19738-19744.

Piura B, Meirovitz M, Shmulman M, Yanai-Inbar I, Cohen Y, Dgani R. Uterine banillary serous carcinoma: studv of 19 cases. Eur J Obstet Gvnecol Reprod Biol $1998 ; 79(1): 69-73$ 
Podratz KC, Mariani A. Uterine papillary serous carcinomas: the exigency for clinical trials. Gynecol Oncol 2003; 91(3):461-462.

Polakis P. Wnt signaling and cancer. Genes Dev 2U00; 14(15):183/-18১1.

Polette M, Nawrocki B, Gilles C, Sato H, Seiki M, Tournier JM et al. MT-MMP expression and localisation in human lung and breast cancers. Virchows Arch 1996; 428(1):29-35.

Prenzel N, Fischer OM, Streit S, Hart S, Ullrich A. The epidermal growth factor receptor family as a central element for cellular signal transduction and diversification. Endocr Relat Cancer 2001; 8(1):11-31.

Price JT, Bonovich MT, Kohn EC. Biochemistry of cancer dissemination. Crit Rev Biochem Mol Biol 1997; 37(3):175-253.

Price $F V$, Chambers sK, Carcangiu MiL, Konom Li, Schwartz FL, Cnambers $j$ i. Intravenous cisplatin, doxorubicin, and cyclophosphamide in the treatment of uterine papillary serous carcinoma (UPSC). Gynecol Oncol 1993; 51(3):383-389.

Quackenbush E, Clabby M, Gottesdiener KM, Barbosa J, Jones NH, Strominger JL et al. Molecular cloning of complementary DNAs encoding the heavy chain of the human 4F2 cell-surface antigen: a type II membrane glycoprotein involved in normal and neoplastic cell growth. Proc Natl Acad Sci U S A 1987; 84(18):6526-6530.

Ramondetta L, Burke TW, Levenback C, Bevers M, Bodurka-Bevers D, Gershenson DM. Treatment of uterine papillary serous carcinoma with paclitaxel. Gynecol Oncol $2001 ; 82(1): 156-161$.

Raz A, Iotan R. Lectin-like activities associated with human and murine neoplastic cells. Cancer Res 1981: 41(9 Pt 1):3642-3647. 
Raz A, Lotan R. Endogenous galactoside-binding lectins: a new class of functional tumor cell surface molecules related to metastasis. Cancer Metastasis Rev 1987; 6(3):433-452.

Raz A, Zhu DG, Hogan V, Shah N, Raz T, Karkash R et al. Evidence for the role of 34-kDa galactoside-binding lectin in transformation and metastasis. Int J Cancer 1990; 46(5):871-877.

Reiss M. TGF-beta and cancer. Microbes Infect 1999; 1(15):1327-1347.

Rintoul RC, Buttery RC, Mackinnon AC, Wong WS, Mosher D, Haslett C et al. Cross-linking CD98 promotes integrin-like signaling and anchorage-independent growth. Mol Biol Cell 2002; 13(8):2841-2852.

Risinger JI, Berchuck A, Kohler MF, Boyd J. Mutations of the E-cadherin gene in human gynecologic cancers. Nat Genet 1994; 7(1):98-102.

Robboy SJ, Anderson M.C., Russell P. Endometrial Carcinoma. Pathology of the Female Reproductive Tract. [1st Edn], 331-356. Edinburgh, Churchill Livingstone.

Rodgers WH, Matrisian LM, Giudice LC, Dsupin B, Cannon P, Svitek C et al. Patterns of matrix metalloproteinase expression in cycling endometrium imply differential functions and regulation by steroid hormones. J Clin Invest 1994; 94(3):946-953.

Rodgers WH, Osteen KG, Matrisian LM, Navre M, Giudice LC, Gorstein F. Expression and localization of matrilysin, a matrix metalloproteinase, in human endometrium during the reproductive cycle. Am I Obstet Gynecol 1993; $168(1 \mathrm{Pt}$ 1):253-260 
Ropponen KM, Eskelinen MJ, Lipponen PK, Alhava EM, Kosma VM. Reduced expression of alpha catenin is associated with poor prognosis in colorectal carcinoma. J Clin Pathol 1999; 52(1):10-16.

Rosen DG, Huang X, Deavers MT, Malpica A, Silva EG, Liu J. Validation of tissue microarray technology in ovarian carcinoma. Mod Pathol 2004; 17(7):790-797.

Rossier G, Meier C, Bauch C, Summa V, Sordat B, Verrey F et al. LAT2, a new basolateral 4F2hc/CD98-associated amino acid transporter of kidney and intestine. J Biol Chem 1999; 274(49):34948-34954.

Rubin MA, Dunn R, Strawderman M, Pienta KJ. Tissue microarray sampling strategy for prostate cancer biomarker analysis. Am J Surg Pathol 2002; 26(3):312319.

Rubinfeld B, Albert I, Porfiri E, Munemitsu S, Polakis P. Loss of beta-catenin regulation by the APC tumor suppressor protein correlates with loss of structure due to common somatic mutations of the gene. Cancer Res 1997; 57(20):4624-4630.

Saito T, Nishimura M, Yamasaki H, Kudo R. Hypermethylation in promoter region of E-cadherin gene is associated with tumor dedifferention and myometrial invasion in endometrial carcinoma. Cancer 2003; 97(4):1002-1009.

Sakuragi N, Nishiya M, Ikeda K, Ohkouch T, Furth EE, Hareyama H et al. Decreased E-cadherin expression in endometrial carcinoma is associated with tumor dedifferentiation and deep myometrial invasion. Gynecol Oncol 1994; 53(2):183189.

Sanders DS, Perry I, Hardy R, Jankowski J. Aberrant P-cadherin expression is a feature of clonal expansion in the gastrointestinal tract associated with repair and neoplasia. I Pathol 2000; 190(5):526-530 
Santin AD. HER2/neu overexpression: has the Achilles' heel of uterine serous papillary carcinoma been exposed?. Gynecol Oncol 2003; 88(3):263-265.

Santin AD, Bellone S, Gokden M, Palmieri M, Dunn D, Agha J et al. Overexpression of HER-2/neu in uterine serous papillary cancer. Clin Cancer Res 2002; 8(5):12711279.

Santin AD, Bellone S, siegei EK, Yaimier M, i homas M, Lannon MH et ai. Kacial differences in the overexpression of epidermal growth factor type II receptor (HER2/neu): a major prognostic indicator in uterine serous papillary cancer. Am J Obstet Gynecol 2005; 192(3):813-818.

Santin AD, Bellone S, Van Stedum S, Bushen W, Las Casas LE, Korourian S et al. Determination of HER2/neu status in uterine serous papillary carcinoma: Comparative analysis of immunohistochemistry and fluorescence in situ hybridization. Gynecol Oncol 2005; 98(1):24-30.

Santin AD, Bellone S, Van Stedum S, Bushen W, Palmieri M, Siegel ER et al. Amplification of c-erbB2 oncogene: a major prognostic indicator in uterine serous papillary carcinoma. Cancer 2005; 104(7):1391-1397.

Santin AD, Diamandis EP, Bellone S, Marizzoni M, Bandiera E, Palmieri M et al. Overexpression of kallikrein 10 (hK10) in uterine serous papillary carcinomas. Am $\mathbf{J}$ Obstet Gynecol 2006; 194(5):1296-1302.

Santin AD, Diamandis EY, Bellone S, Soosaipiliar A, Cane S, Paimierı Mi et ai. Human kallikrein 6: a new potential serum biomarker for uterine serous papillary cancer. Clin Cancer Res 2005; 11(9):3320-3325.

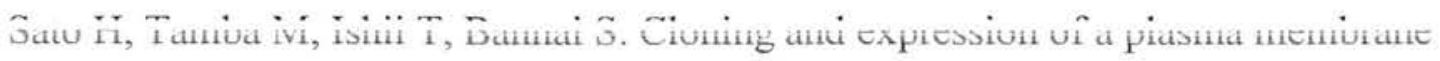
cystine/glutamate exchange transporter composed of two distinct proteins. J Biol Chem 1Y99; 2/4(17):11450-11438. 
Sato S, Hughes RC. Regulation of secretion and surface expression of Mac-2, a galactoside-binding protein of macrophages. J Biol Chem 1994; 269(6):4424-4430.

Satoh S, Daigo Y, Furukawa Y, Kato T, Miwa N, Nishiwaki T et al. AXIN1 mutations in hepatocellular carcinomas, and growth suppression in cancer cells by virus-mediated transfer of AXIN1. Nat Genet 2000; 24(3):245-250.

Scambia G, Testa U, Benedetti PP, Foti E, Martucci R, Gadducci A et al. Prognostic significance of interleukin 6 serum levels in patients with ovarian cancer. $\mathrm{Br} \mathrm{J}$ Cancer $1995 ; 71(2): 354-356$.

Schmitz MJ, Hendricks DT, Farley J, Taylor RR, Geradts J, Rose GS et al. p27 and cyclin D1 abnormalities in uterine papillary serous carcinoma. Gynecol Oncol 2000; $77(3): 439-445$.

Scully RE, Bonfiglio TA, Kurman RJ. Histological Typing of Female Genital Tract Tumours. World Health Organisation International Histological Classification of Tumours. [2nd Edn], 13-15. Berlin, Springer-Verlag.

Segawa H, Fukasawa Y, Miyamoto K, Takeda E, Endou H, Kanai Y. Identification and functional characterization of a $\mathrm{Na}+$-independent neutral amino acid transporter with broad substrate selectivity. J Biol Chem 1999; 274(28):19745-19751.

Segawa T, Shozu M, Murakami K, Kasai T, Shinohara K, Nomura K et al. Aromatase expression in stromal cells of endometrioid endometrial cancer correlates with poor survival. Clin Cancer Res 2005; 11(6):2188-2194.

Sherman ME, Bitterman P, Rosenshein NB, Delgado G, Kurman RJ. Uterine serous carcinoma. A morphologically diverse neoplasm with unifying clinicopathologic features. Am J Surg Pathol 1992; 16(6):600-610.

Shi Y. Structural insights on Smad function in TGFbeta signaling. Bioessays 2001; 23(3):223-232. 
Shih HC, Shiozawa T, Miyamoto T, Kashima H, Feng YZ, Kurai M et al. Immunohistochemical expression of E-cadherin and beta-catenin in the normal and malignant human endometrium: an inverse correlation between E-cadherin and nuclear beta-catenin expression. Anticancer Res 2004; 24(6):3843-3850.

Shimazui T, Bringuier PP, van Berkel H, Ruijter E, Akaza H, Debruyne FM et al. Decreased expression of alpha-catenin is associated with poor prognosis of patients with localized renal cell carcinoma. Int J Cancer 1997; 74(5):523-528.

Shiraga M, Yano S, Yamamoto A, Ogawa H, Goto H, Miki T et al. Organ heterogeneity of host-derived matrix metalloproteinase expression and its involvement in multiple-organ metastasis by lung cancer cell lines. Cancer Res 2002; 62(20):5967-5973.

Shore EM, Nelson WJ. Biosynthesis of the cell adhesion molecule uvomorulin (Ecadherin) in Madin-Darby canine kidney epithelial cells. J Biol Chem 1991; 266(29):19672-19680.

Siegel PM, Massague J. Cytostatic and apoptotic actions of TGF-beta in homeostasis and cancer. Nat Rev Cancer 2003; 3(11):807-821.

Skacel M, Skilton B, Pettay JD, Tubbs RR. Tissue microarrays: a powerful tool for high-throughput analysis of clinical specimens: a review of the method with validation data. Appl Immunohistochem Mol Morphol 2002; 10(1):1-6.

Slomovitz BM, Broaddus RR, Schmandt R, Wu W, Oh JC, Ramondetta LM et al. Expression of imatinib mesylate-targeted kinases in endometrial carcinoma. Gynecol Oncol 2004; 95(1):32-36.

Slomovitz BM, Burke TW, Eifel PJ, Ramondetta LM, Silva EG, Jhingran A et al. Uterine papillary serous carcinoma (UPSC): a single institution review of 129 cases Gynecol Oncol 2003: 91(3):463-469 
Smythe WR, Williams JP, Wheelock MJ, Johnson KR, Kaiser LR, Albelda SM. Cadherin and catenin expression in normal human bronchial epithelium and nonsmall cell lung cancer. Lung Cancer 1999; 24(3):157-168.

Sood BM, Jones J, Gupta S, Khabele D, Guha C, Runowicz C et al. Patterns of failure after the multimodality treatment of uterine papillary serous carcinoma. Int $\mathbf{J}$ Radiat Oncol Biol Phys 2003; 57(1):208-216.

Spiegel GW. Endometrial carcinoma in situ in postmenopausal women. Am J Surg Pathol 1995; 19(4):417-432.

Stamenkovic I. Matrix metalloproteinases in tumor invasion and metastasis. Semin Cancer Biol 2000; 10(6):415-433.

Stetler-Stevenson WG. Matrix metalloproteinases in angiogenesis: a moving target for therapeutic intervention. J Clin Invest 1999; 103(9):1237-1241.

Still K, Robson CN, Autzen P, Robinson MC, Hamdy FC. Localization and quantification of mRNA for matrix metalloproteinase-2 (MMP-2) and tissue inhibitor of matrix metalloproteinase-2 (TIMP-2) in human benign and malignant prostatic tissue. Prostate 2000; 42(1):18-25.

Sutton GP, Brill L, Michael H, Stehman FB, Ehrlich CE. Malignant papillary lesions of the endometrium. Gynecol Oncol 1987; 27(3):294-304.

Swisher EM, Mutch DG, Rader JS, Elbendary A, Herzog TJ. Topotecan in platinumand paclitaxel-resistant ovarian cancer. Gynecol Oncol 1997; 66(3):480-486.

Sykiotis K, Kondi-Pafiti A, Giannaris D, Kouvaris J, Papayannopoulou A, Salamalekis E. Uterine serous papillary carcinoma clinical and immunopathological study of 9 cases. Eur J Gynaecol Oncol 2001; 22(6):456-458. 
Takeichi M. Cadherin cell adhesion receptors as a morphogenetic regulator. Science 1991; 251(5000):1451-1455.

Tamiolakis D, Venizelos J, Lambropoulou M, Nikolaidou S, Tsikouras P, Jivannakis $\mathrm{T}$ et al. Local immune response in serous papillary carcinoma of the endometrium. Histol Histopathol 2005; 20(2):403-408.

Taniuchi K, Nakagawa H, Hosokawa M, Nakamura T, Eguchi H, Ohigashi H et al. Overexpressed $\mathrm{P}$-cadherin/CDH3 promotes motility of pancreatic cancer cells by interacting with p120ctn and activating rho-family GTPases. Cancer Res 2005; 65(8):3092-3099.

Tavassoli F, Devilee P. Tumours of the Breast and Female Genital Organs. 217-257. 2006. Lyon, IARCPress.

Tay EH, Ward BG. The treatment of uterine papillary serous carcinoma (UPSC): are we doing the right thing?. Int J Gynecol Cancer 1999; 9(6):463-469.

Teixeira S, Di Grandi S, Kuhn LC. Primary structure of the human 4F2 antigen heavy chain predicts a transmembrane protein with a cytoplasmic $\mathrm{NH} 2$ terminus. $\mathrm{J}$ Biol Chem 1987; 262(20):9574-9580.

Torhorst J, Bucher C, Kononen J, Haas P, Zuber M, Kochli OR et al. Tissue microarrays for rapid linking of molecular changes to clinical endpoints. Am J Pathol 2001; 159(6):2249-2256.

Torrents D, Estevez R, Pineda M, Fernandez E, Lloberas J, Shi YB et al. Identification and characterization of a membrane protein $(y+L$ amino acid transporter-1) that associates with $4 \mathrm{~F} 2 \mathrm{hc}$ to encode the amino acid transport activity $\mathrm{y}+$ L. A candidate gene for lysinuric protein intolerance. J Biol Chem 1998; 273(49):32437-32445. 
Tsurudome M, Ito M, Takebayashi S, Okumura K, Nishio M, Kawano M et al. Cutting edge: primary structure of the light chain of fusion regulatory protein$1 / \mathrm{CD} 98 / 4 \mathrm{~F} 2$ predicts a protein with multiple transmembrane domains that is almost identical to the amino acid transporter E16. J Immunol 1999; 162(5):2462-2466.

Tunuguntla R, Ripley D, Sang QX, Chegini N. Expression of matrix metalloproteinase-26 and tissue inhibitors of metalloproteinases TIMP-3 and -4 in benign endometrium and endometrial cancer. Gynecol Oncol 2003; 89(3):453-459.

Ueno H, Yamashita K, Azumano I, Inoue M, Okada Y. Enhanced production and activation of matrix metalloproteinase-7 (matrilysin) in human endometrial carcinomas. Int J Cancer 1999; 84(5):470-477.

Umbas R, Isaacs WB, Bringuier PP, Schaafsma HE, Karthaus HF, Oosterhof GO et al. Decreased E-cadherin expression is associated with poor prognosis in patients with prostate cancer. Cancer Res 1994; 54(14):3929-3933. Umpierre SA, Burke TW, Tornos C, Ordonez N, Levenback C, Morris M. Immunocytochemical analysis of uterine papillary serous carcinomas for estrogen and progesterone receptors. Int J Gynecol Pathol 1994; 13(2):127-130.

van de WM, Barker N, Harkes IC, van der HM, Dijk NJ, Hollestelle A et al. Mutant E-cadherin breast cancer cells do not display constitutive Wnt signaling. Cancer Res $2001 ; 61(1): 278-284$.

van den Brule FA, Buicu C, Berchuck A, Bast RC, Deprez M, Liu FT et al. Expression of the $67-\mathrm{kD}$ laminin receptor, galectin-1, and galectin-3 in advanced human uterine adenocarcinoma. Hum Pathol 1996; 27(11):1185-1191.

van der Linden PJ, de Goeij AF, Dunselman GA, Arends JW, Evers JL. P-cadherin expression in human endometrium and endometriosis. Gynecol Obstet Invest 1994; 38(3):183-185. 
Van Roy F, Mareel M. Tumour invasion: effects of cell adhesion and motility. Trends Cell Biol 1992; 2(6):163-169.

Vasil'eva EV, Belianin VL. Serous adenocarcinoma of the uterus: criteria of morphological diagnosis and immunohistochemistry. Arkh Patol 2005; 67(2):25-27.

Vihinen P, Kahari VM. Matrix metalloproteinases in cancer: prognostic markers and therapeutic targets. Int J Cancer 2002; 99(2):157-166.

Vizirianakis IS, Yao CC, Chen Y, Ziober BL, Tsiftsoglou AS, Kramer RH.

Transfection of MCF-7 carcinoma cells with human integrin alpha7 cDNA promotes adhesion to laminin. Arch Biochem Biophys 2001; 385(1):108-116.

Vogeimann K, Nguyen-1 at MD, Giehi K, Adler G, Wedich D, Menke A. IGH betainduced downregulation of E-cadherin-based cell-cell adhesion depends on PI3kinase and PTEN. J Cell Sci 2005; 118(Pt 20):4901-4912.

Voutilainen KA, Anttila MA, Sillanpaa SM, Ropponen KM, Saarikoski SV, Juhola MT et al. Prognostic significance of E-cadherin-catenin complex in epithelial ovarian cancer. J Clin Pathol 2006; 59(5):460-467.

Wakefield LM, Roberts AB. TGF-beta signaling: positive and negative effects on tumorigenesis. Curr Opin Genet Dev 2002; 12(1):22-29.

Walker AN, Mills SE. Serous papillary carcinoma of the endometrium. A clinicopathologic study of 11 cases. Diagn Gynecol Obstet 1982; 4(4):261-267.

Wheeler DT, Bell KA, Kurman RJ, Sherman ME. Minimal uterine serous carcinoma: diagnosis and clinicopathologic correlation. Am J Surg Pathol 2000; 24(6):797-806.

Witty JP, McDonnell S, Newell KJ, Cannon P, Navre M, Tressler RJ et al. Modulation of matrilysin levels in colon carcinoma cell lines affects tumorigenicity in vivo. Cancer Res 1994; 54(17):4805-4812. 
Wong SF, Lai LC. The role of TGFbeta in human cancers. Pathology 2001; $33(1): 85-92$.

Wu W, Slomovitz BM, Celestino J, Chung L, Thornton A, Lu KH. Coordinate expression of Cdc25B and ER-alpha is frequent in low-grade endometrioid endometrial carcinoma but uncommon in high-grade endometrioid and nonendometrioid carcinomas. Cancer Res 2003; 63(19):6195-6199.

Xu X, Wang Y, Chen Z, Sternlicht MD, Hidalgo M, Steffensen B. Matrix metalloproteinase- 2 contributes to cancer cell migration on collagen. Cancer Res $2005 ; 65(1): 130-136$.

Yabushita H, Narumiya H, Hiratake K, Yamada H, Shimazu M, Sawaguchi K et al. The association of transforming growth factor-beta 1 with myometrial invasion of endometrial carcinomas through effects on matrix metalloproteinase. J Obstet Gynaecol Res 2000; 26(3):163-170.

Yagita H, Masuko T, Hashimoto Y. Inhibition of tumor cell growth in vitro by murine monoclonal antibodies that recognize a proliferation-associated cell surface antigen system in rats and humans. Cancer Res 1986; 46(3):1478-1484.

Yang RY, Hill PN, Hsu DK, Liu FT. Role of the carboxyl-terminal lectin domain in self-association of galectin-3. Biochemistry 1998; 37(12):4086-4092.

Yang SZ, Kohno N, Yokoyama A, Kondo K, Hamada H, Hiwada K. Decreased Ecadherin augments beta-catenin nuclear localization: studies in breast cancer cell lines. Int J Oncol 2001; 18(3):541-548.

Yao CC, Ziober BL, Squillace RM, Kramer RH. Alpha7 integrin mediates cell adhesion and migration on specific laminin isoforms. J Biol Chem 1996; 271(41):25598-25603. 
Yin XM, Oltvai ZN, Korsmeyer SJ. BH1 and BH2 domains of Bcl-2 are required for inhibition of apoptosis and heterodimerization with Bax. Nature 1994; 369(6478):321-323.

Yu F, Finley RL, Jr., Raz A, Kim HR. Galectin-3 translocates to the perinuclear membranes and inhibits cytochrome $\mathrm{c}$ release from the mitochondria. A role for synexin in galectin-3 translocation. J Biol Chem 2002; 277(18):15819-15827.

Yu Q, Stamenkovic I. Cell surface-localized matrix metalloproteinase-9 proteolytically activates TGF-beta and promotes tumor invasion and angiogenesis. Genes Dev 2000; 14(2):163-176.

Zaika AI, Slade N, Erster SH, Sansome C, Joseph TW, Pearl M et al. DeltaNp73, a dominant-negative inhibitor of wild-type p53 and TAp73 is upregulated in human tumours. J Exp Med 2002; 196(6):765-780.

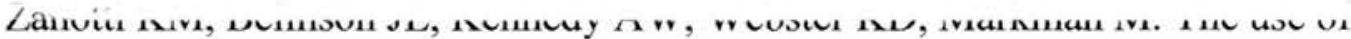
paclitaxel and platinum-based chemotherapy in uterine papillary serous carcinoma. Gynecol Oncol 1999; 74(2):272-277.

Zhao XJ, Li H, Chen H, Liu YX, Zhang LH, Liu SX et al. Expression of e-cadherin and beta-catenin in human esophageal squamous cell carcinoma: relationships with prognosis. World J Gastroenterol 2003; 9(2):225-232.

Zheng W, Khurana R, Farahmand S, Wang Y, Zhang ZF, Felix JC. p53 immunostaining as a significant adjunct diagnostic method for uterine surface carcinoma: precursor of uterine papillary serous carcinoma. Am J Surg Pathol 1998; 22(12):1463-1473.

Zheng W, Liang SX, Yu H, Rutherford T, Chambers SK, Schwartz PE. Endometrial glandular dysplasia: a newly defined precursor lesion of uterine papillary serous carcinoma. Part I: morphologic features. Int J Surg Pathol 2004; 12(3):207-223. 
Zhou YN, Xu CP, Han B, Li M, Qiao L, Fang DC et al. Expression of E-cadherin and beta-catenin in gastric carcinoma and its correlation with the clinicopathological features and patient survival. World J Gastroenterol 2002; 8(6):987-993.

Ziober BL, Lin CS, Kramer RH. Laminin-binding integrins in tumour progression and metastasis. Semin Cancer Biol 1996; 7(3):119-128.

Zorn KK, Bonome T, Gangi L, Chandramouli GV, Awtrey CS, Gardner GJ et al. Gene expression profiles of serous, endometrioid, and clear cell subtypes of ovarian and endometrial cancer. Clin.Cancer Res. 11[18], 6422-6430. 15-9-2005.

Zukerberg LR, DeBernardo RL, Kirley SD, D'Apuzzo M, Lynch MP, Littell RD et al. Loss of cables, a cyclin-dependent kinase regulatory protein, is associated with the development of endometrial hyperplasia and endometrial cancer. Cancer Res 2004; 64(1):202-208. 


\section{Appendix 1}

The following publications resulted from the work of this thesis:

Faratian D, Stillie A, Busby-Earle RMC, Cowie VJ, Monaghan H. A review of the pathology and management of uterine papillary serous carcinoma and correlation with outcome. Int. J. Gynecol. Cancer 2006;16:972-978.

Monaghan H, Williams ARW. The pattern of CD98 expression is different between uterine serous papillary carcinoma and endometrioid endometrial carcinoma. Gynecol Oncol. 2007;104(1):264-5.

H Monaghan, N MacWhinnie, ARW Williams. The Role of Matrix Metalloproteases -2, -7 and -9 and $\beta$ Catenin in High Grade Endometrial Carcinoma. Histopathology. 2007 50(3):348-57. 


\title{
A review of the pathology and management of uterine papillary serous carcinoma and correlation with outcome
}

\author{
D. FARATIAN*, A. STILLIE†, R.M. C. BUSBY-EARLE $\ddagger$, V.J. COWIE† \& H. MONAGHAN* \\ *Department of Laboratory Services (Pathology), Royal Infirmary of Edinburgh, Edinburgh, United Kingdom; \\ tEdinburgh Cancer Centre, Western General Hospital, Edinburgh, United Kingdom; and ¥Simpson Centre \\ for Reproductive Health, Royal Infirmary of Edinburgh, Edinburgh, United Kingdom
}

\begin{abstract}
Faratian D, Stillie A, Busby-Earle RMC, Cowie VJ, Monaghan H. A review of the pathology and management of uterine papillary serous carcinoma and correlation with outcome. Int $J$ Gynecol Cancer 2006;16:972-978.

Uterine papillary serous carcinoma (UPSC) accounts for $10 \%$ of endometrial carcinomas but a higher proportion of deaths due to its aggressive nature and poor response to chemotherapy and radiotherapy. In order to add to the knowledge of UPSC in the literature and to review our local practices, we examined the pathology, medical records, and management of all cases of UPSC (67 patients) treated in South East Scotland over a 10-year period and also evaluated the prognostic significance of the percentage of UPSC in endometrial pipelle and hysterectomy specimens. Although only $63 \%$ of initial diagnostic biopsies were reported to contain UPSC, rereview of the cases revealed UPSC in $98.5 \%$ of the preoperative biopsies. The percentage of UPSC in the tumors did not affect the outcome. Stage, positive omentum, and treatment with external-beam +1- intracavitary radiotherapy were significantly correlated with overall survival and progression-free survival by univariate analysis, but only stage $(P<0.01)$ was correlated with outcome on multivariate analysis. Chemotherapy did not affect outcome. UPSC may be difficult to diagnose in preoperative biopsies, particularly when present as part of a mixed tumor. Even a small percentage of UPSC in a diagnostic biopsy or hysterectomy specimen is correlated with a poor prognosis. This study emphasizes the need of a cooperative, prospective study on this distinct uterine carcinoma.
\end{abstract}

KEYWORDS: chemotherapy, papillary, radiotherapy, serous, uterus.

While papillary morphology has been recognized in endometrial carcinoma since the turn of the past century, uterine papillary serous carcinoma (UPSC) was first described as a distinct clinical entity in 1982 by Lauchlan $^{(1)}$ and Hendrickson et al. ${ }^{(2,3)}$ UPSC usually occurs in elderly, postmenopausal women and, in contrast to endometrioid endometrial carcinoma (EEC), is not associated with excess estrogen. There is conflicting data regarding the association of breast cancer with high-risk endometrial carcinomas ${ }^{(4,5)}$. It is thought

Address correspondence and reprint request to: $\mathrm{Dr}$ Hannah Monaghan, MBBS BSc, Department of Laboratory Services (Pathology), Royal Infirmary of Edinburgh, 51 Little France Crescent, Edinburgh EH16 4SA, UK. Email: hannah.monaghan@luht.scot.nhs.uk

None of the authors had any conflict of interests regarding the present work. that patients with breast cancer have a similar rate of low- and high-risk endometrial subtypes irrespective of tamoxifen use. However, patients with UPSC may have an increased risk of synchronous or subsequent development of breast cancer ${ }^{(6,7)}$. This raises the possibility that UPSC and breast cancer may, in some patients, be due to the presence of mutations in cancer predisposing genes such as BRCA $1^{(8)}$.

Although accounting for only $10 \%$ of endometrial carcinomas, UPSC is an important diagnosis to make on initial biopsy since it behaves particularly aggressively, with a propensity for early metastasis that results in upstaging at the time of operation in $50-75 \%$ of clinical stage I cancers ${ }^{(9-11)}$. This underscores the need for accurate preoperative diagnosis, so that the surgeon can plan definitive treatment and perform accurate and thorough surgical staging by the FIGO 
system, including a total extrafascial hysterectomy and bilateral salpingo-oophorectomy, peritoneal washings, and removal of any suspicious pelvic or paraaortic lymph nodes. Although omentectomy is not in the 1988 FICO system for staging, it is also frequently performed. This is because UPSC behaves aggres sively and has a pattern of spread similar to that of ovarian serous carcinoma. There is no evidence to date that this radical surgery affects outcome, but accurate staging may help influence current advice regarding adjuvant therapy. UPSC may exist in both "pure" and "mixed" forms with other endometrial cancer subtypes, in particular EEC and clear cell endometrial carcinoma (CCEC). Sherman et al. ${ }^{(12)}$ state that the diagnosis of UPSC should be reserved for cancers with more than 25\% UPSC pattern in the final resection specimen, although this figure appcars arbitrary.

Data as to the optimum management of UPSC are limited due to its relative rarity. This audit was performed to look at the patient characteristics, histopathologic characteristics, and outcomes of various treatment regimens of UPSC. In particular, we wished to determinte the accuracy of diagnusing UTSC un endometrial pipelle biopsies or curettings, whether the histologic subtypes seen in the diagnostic biopsy correlated with those seen in the final resection specimen, and also what percentage of UPSC pattern is required to confer the poor prognostic phenotype. To date, there have been several studies looking at either the onco logic or the surgical management of UPSC, ranging from 9 to 129 cases $^{(3,6,9,13-18)}$. Our review of the management of this challenging disease is the fourth largest of its kind (67 patients), and to our knowledge, the first to address these specific pathologic questions.

\section{Materials and methods}

Between 1994 and 2003, 67 patients had a tissue diagnosis of pure UPSC or a mixed carcinoma containing a UPSC component made on initial biopsy or in the resection specimen. All these cases were reviewed, and the management was discussed at the regional gynecological oncology clinicopathologic meeting. Of these, 59 patients had surgical intervention, and 57 received adjuvant therapy of radiotherapy $+/-$ chemotherapy.

The patient demographics, histopathology, and treatment were recorded.

\section{Patient characteristics}

Surgical and oncologic medical records were reviewed to obtain information about patients' age at presentation, any previous history of breast cancer, the treat- ment, and follow up data, in particular the site of any relapse. The primary end points were progression-free survival (PFS) and overall survival (OS), defined as the period from the date of diagnosis to the date of recurrence or the last clinic visit (if alive) or the date of death. Data were censored if information on survival was not available or the patient had ceased to be followed up for any reason but had not died of cancer.

\section{Histopathologic characteristics}

All diagnostic biopsies and hysterectomy sections from the 67 patients were reviewed by two patholo gists (D.F. and H.M.). The diagnosis of UPSC, CCEC, and EEC were made using the classical microscopic features, which are well known. The pipelle or endometrial curettings were reviewed to determine if definitive diagnosis of UPSC was possible on the original biopsy. Then, the hysterectomy specimens were reviewed to determine if it was a pure UPSC or mixed tumor. If it was a mixed tumor, the percentage of UPSC, EEC, and CCEC of the total tumor present was estimated in the tissue processed for histulugic andysis. Estimates were made by counting low-power $(\times 20-\times 40)$ fields of each pattern and dividing this by the total number of fields. Data were compared, and the three discrepancies resolved by case conference. The number and nature of specimens additional to the hysterectomy specimen received (ic, omentum, pelvic lymph nodes, or peritoneal washings) were noted, along with the pathologic stage according to the 1988 FIGO surgical staging criteria at the time of resection.

\section{Oncologic characteristics}

The adjuvant therapy was recorded, and the chemotherapy and radiotherapy regimens (external beam + / - vaginal cesium) were documented. Where the data were available, details of the treatment of recurrence were also noted.

\section{Statistical analysis}

All statistical analyses were performed using SPSS software (SPSS Inc, Chicago, IL). The Spearman's rank test was used to assess the correlation of the initial diagnostic biopsy with the definitive hysterectomy histology percentage histologic subtype. OS and PFS were estimated on the basis of Kaplan-Meier curves and the logrank test used as a univariate analysis of prognostic factors. A $P$ value of less than 0.05 was considered statistically significant, and variables that had a $P$ value of less than 0.05 were entered into a Cox regression model. 


\section{Results}

\section{Patient characteristics}

The median age of the patients was 68 years (range $49-89$ years). Nine patients (13\%) had a medical history of breast cancer. No patients developed breast cancer after the time of diagnosis of UPSC. The median follow-up for all patients was 14.5 months (range 1-114 months). The numbers and percentages of each patient with each stage of disease are shown in Table 1, along with 1-year and 3-year OS and PFS by stage. OS and PFS correlated significantly with the stage of disease ( $P<0.01$; Fig. 1$)$, the median OS was $67,43,14$, and 9 months for stage I, II, III, and IV disease, respectively. The median PFS for stage I disease was not reached in this study, due to censoring, but the median PFS for stage II, III, and IV disease was 43, 9 , and 8 months, respectively.

\section{Surgical analysis}

Fifty-nine patients underwent surgery. All these patients underwent a total abdominal hysterectomy, bilateral salpingo-oophorectomy. Twenty-four patients $(40.1 \%)$ had, in addition, omental sampling, 21 patients (87.5\%) an omental biopsy, and 3 patients (12.5\%) full omentectomy. Seven patients $(11.9 \%$ ) had a pelvic node clearance; in 30 patients $(50.9 \%)$, peritoneal washings were taken. In 7 patients total abdominal hysterectomy, bilateral salpingo-oophorectomy, omentectomy, and pelvic node sampling were carried out and peritoneal washings were taken. No patients had para-aortic node sampling.

Table 1. PFS and OS by stage

\begin{tabular}{|c|c|c|c|c|c|}
\hline \multirow[b]{2}{*}{ Stage } & \multirow{2}{*}{$\begin{array}{l}\text { Number of } \\
\text { patients (\%) }\end{array}$} & \multicolumn{2}{|l|}{ PFS } & \multicolumn{2}{|l|}{ OS } \\
\hline & & 1 year & 3 years & 1 year & 3 years \\
\hline Stage I & $25(37)$ & 84.7 & $68.2^{*}$ & 88.2 & $64.1^{* *}$ \\
\hline IA & $3(4.5)$ & & & & \\
\hline IB & $14(21)$ & & & & \\
\hline IC & $8(12)$ & & & & \\
\hline Stage II & $14(20)$ & 81.8 & $60.1^{*}$ & 81.8 & $50.9^{* *}$ \\
\hline IIA & $7(10)$ & & & & \\
\hline IIB & $7(10)$ & & & & \\
\hline Stage III & $19(28)$ & 41.7 & $25.0^{*}$ & 56.2 & $21.4^{* *}$ \\
\hline IIIA & $14(21)$ & & & & \\
\hline IIIB & $3(4.5)$ & & & & \\
\hline IIIC & $2(3)$ & & & & \\
\hline Stage IV & $9(13)$ & 0 & ND & 41.6 & ND \\
\hline All patients & 67 & 61.5 & 46.2 & 67.0 & 39.4 \\
\hline
\end{tabular}

ND, no data.

${ }^{*} P<0.01 ;{ }^{* *} P<0.01$.
Seven $(29 \%)$ omental samples were positive for metastatic disease, two $(28.5 \%)$ pelvic lymph node clearances were positive, while six of $30(20 \%)$ of peritoneal washings contained malignant cells. Patients who had a negative omental biopsy at the time of operation showed a significantly better PFS and OS $(P<0.01)$ than those who had a positive biopsy (Fig. 2A). Of the three omentectomies performed, one was positive and two were negative. Positive peritoneal cytology was weakly associated with poor prognosis (log-rank test, $P=0.15$ ), while the effect of pelvic lymphadenectomy on prognosis could not be analyzed due to small numbers of patients.

\section{Histopathologic analysis}

On reviewing the pathology reports, 63 of the cases were diagnosed by pipelle biopsy, curettage, or hysteroscopic biopsy; one case was diagnosed on cervical cytology, and three cases were from hysterectomy histology. Of the 63 patients who had a preoperative diagnostic biopsy, 23 biopsies (36.5\%) showed pure UPSC histology and $39(62 \%)$ showed mixed histologic subtypes including a UPSC component. Thirty nine of the diagnostic biopsies $(63 \%)$ were described as serous, papillary, mixed serous/other histologic subtype in the original pathologic reports, and on review, 62 of the biopsies $(98.5 \%)$ contained a UPSC component. The one biopsy (1.5\%) that did not contain a UPSC component was reviewed as $100 \%$ CCEC, and on hysterectomy, the tumor was reviewed as $100 \%$ UPSC. The correlation between the initial biopsy and the hysterectomy histology subtypes is illustrated in Figure 3 . While there was a weak positive correlation between the diagnostic biopsies and the hysterectomy diagnoses for UPSC and CCEC (Spearman's rank correlation coefficient 0.47 and 0.30 , respectively, $P<$ 0.01 ), the correlation is much stronger for EEC (Spearman's rank correlation coefficient $0.70, P<0.01$ ). In addition, those biopsies, which overestimated the amount of UPSC in the resection, were usually the same cases in which the percentage of CCEC was underestimated. In Kaplan-Meier analyses of survival, the OS and PFS were not significantly different in patients with pure and mixed histologic patterns or percentage of UPSC (including less than 25\% UPSC compared to more than $25 \%$ UPSC) in the diagnostic biopsies or the resections (Fig. 4). In addition, whether UPSC was mixed with EEC or CCEC made no difference to survival. Serous endometrial intraepithelial carcinoma was identified in two of the hysterectomy specimens. 

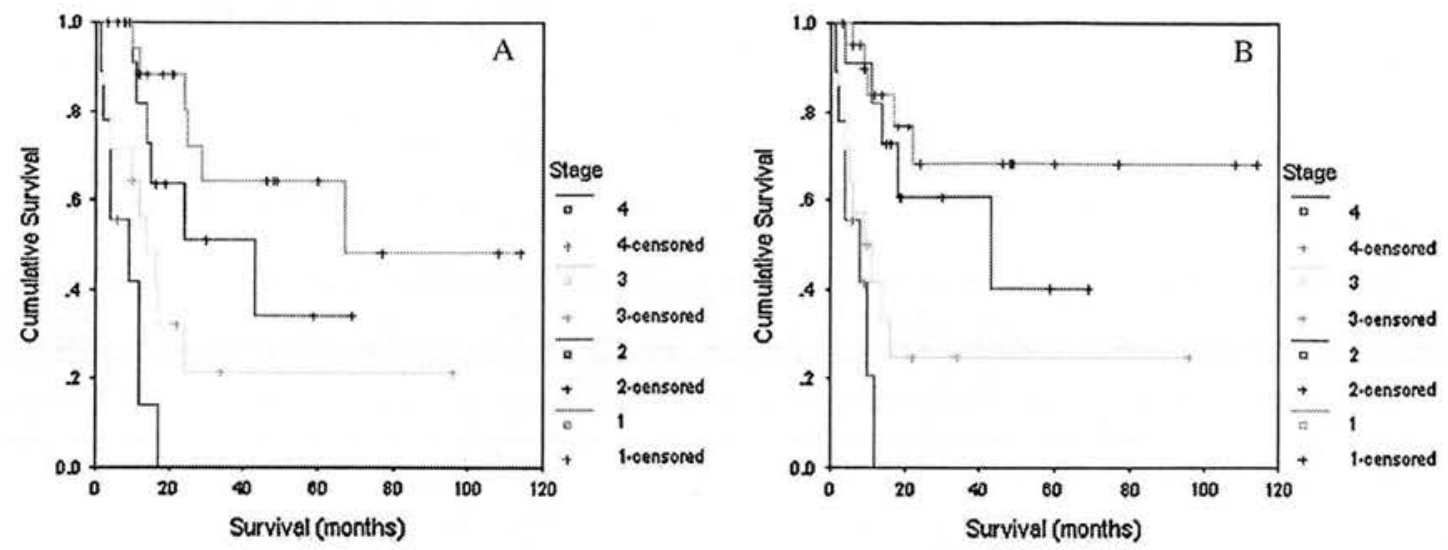

Figure 1. A) OS by stage $(P<0.01)$, and B) PFS by stage $(P<0.01)$.

\section{Adjuvant therapy}

Fifty-seven patients received adjuvant therapy. Thirty patients $(52.6 \%)$ received radical radiotherapy, and three patients $(5.2 \%)$ palliative therapy. Of the two patients who did not receive radiotherapy, one was deemed to have had curative surgery (stage I disease) and the other was deemed unfit for therapy. In addition to radiotherapy, 28 patients $(49.1 \%)$ received adjuvant chemotherapy with different regimens including carboplatin alone, carboplatin and paclitaxel, or
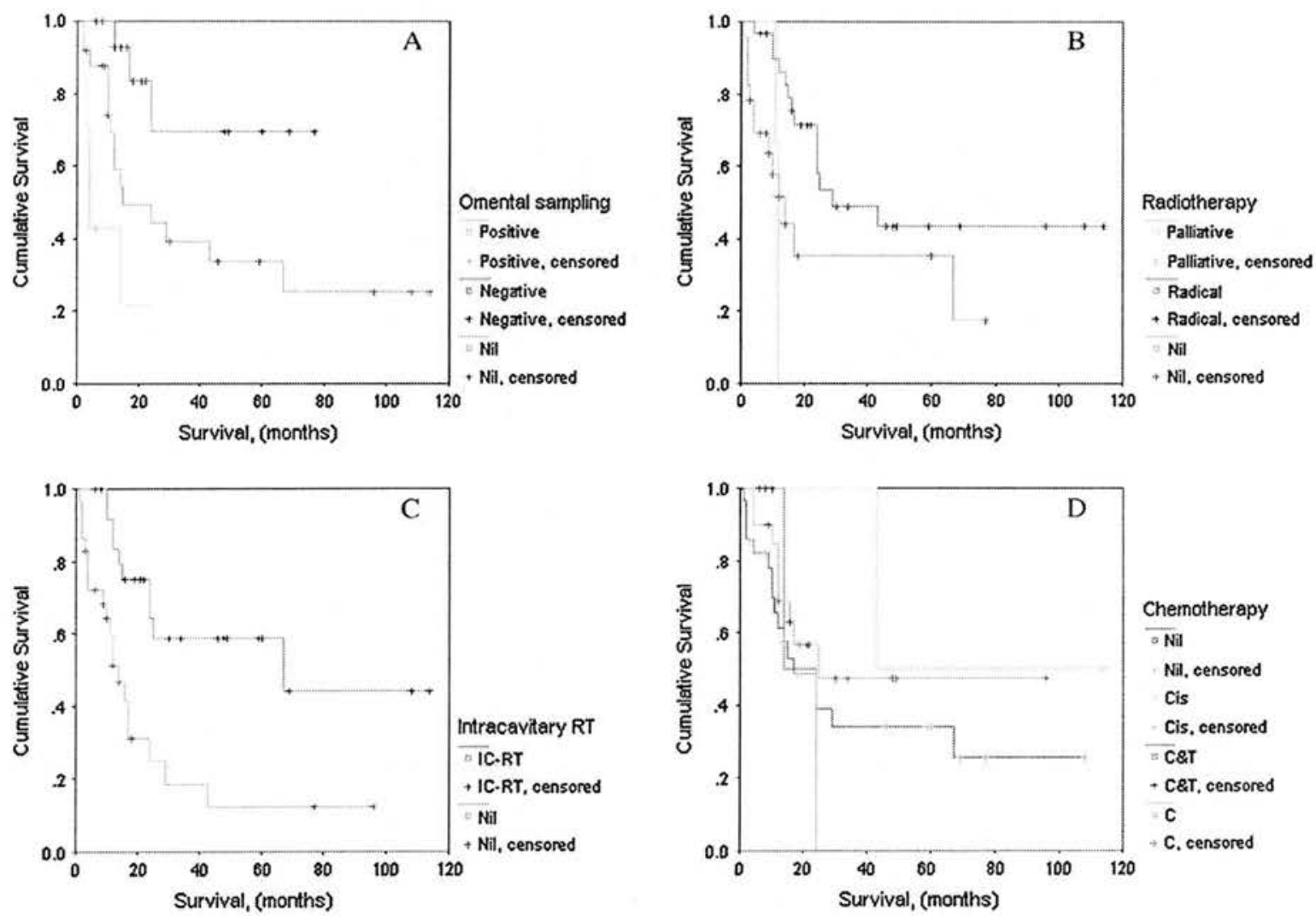

Figure 2. Kaplan-Meier curves of treatment variables by OS. A) Omental sampling $P<0.01$ (log-rank test), B) radiotherapy $P<0.01$ (log-rank test), C) intracavitary radiotherapy $P<0.01$ (log-rank test), and D) chemotherapy $P=0.64$ (log-rank test).RT, radiotherapy; Cis, cisplatinum; C\&T, carboplatin and paclitaxel (Taxol); C, carboplatin. 

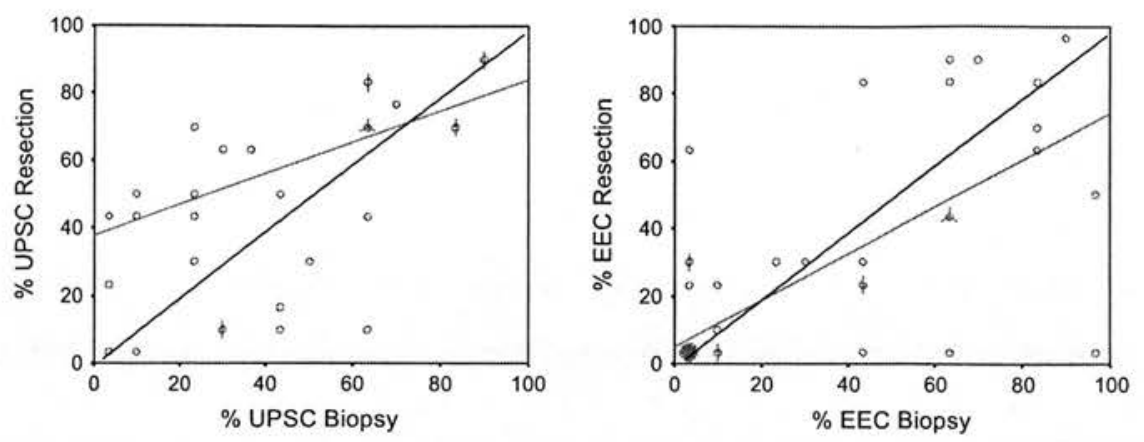

Figure 3. Scatter plots to represent percentages of histologic subtypes in diagnostic biopsies vs resection specimens. Each spoke on a data point represents one case. Best-fit lines (red) show a positive correlation between the two specimens for all three histologic subtypes. cisplatin (Table 2). Patients treated with radical radiotherapy postoperatively had a significantly better prognosis by univariate analysis $(P<0.01$; Fig. $2 B, C)$. The median OS was 29 months in patients receiving radical radiotherapy compared with 12 months in patients not receiving radical radiotherapy, and the median OS was 67 months in patients receiving palliative radiotherapy compared with 12 months in patients not receiving palliative radiotherapy. There were no significant differences in survival in patients treated with or without chemotherapy (Fig. 2D) or between the different chemotherapy regimens.

\section{Recurrence analysis}

Fifteen $(22.4 \%)$ patients had recorded recurrences. Of these, five $(33 \%)$ were distant metastases (lung, liver, omentum, and neck lymph nodes) and ten (67\%) were locoregional recurrences. Median time to recurrence was 9 months (range 4-17 months). Two omental recurrences were in patients who had positive omental biopsies. Neither had underwent complete omentectomy. The recurrence rate in stage I-IV patients was $12 \%, 14 \%, 36 \%$, and $33 \%$, respectively. Further survival analysis was not possible due to the small numbers in each stage and nonstandardized treatment regimens.

\section{Discussion}

This study of UPSC is the third largest retrospective series of its kind with focus on the effect of histopathology on outcome. The high median age of the patients, and postmenopausal status, is similar to that
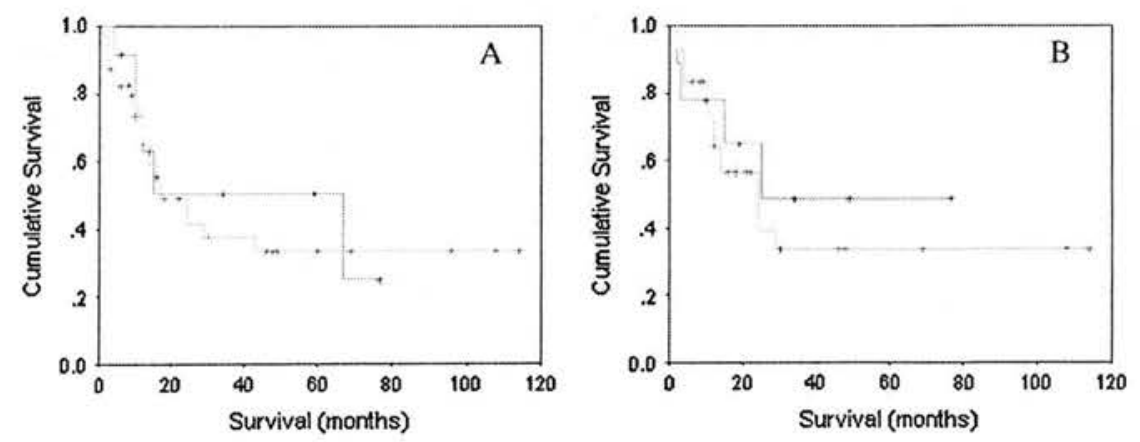

Figure 4. OS. Less than $25 \%$ UPSC (purple line) compared with greater than 25\% UPSC (green line) in preoperative (A, $P=0.76$, log-rank test) and resection specimens $(B, P=0.56$, log-rank test). 
Table 2. Chemotherapy regimens by stage

\begin{tabular}{llllr}
\hline Stage & C & C\&T & Cis & Nil \\
\hline I & 7 & 2 & 1 & 13 \\
II & 4 & 0 & 1 & 6 \\
III & 5 & 3 & 0 & 5 \\
IV & $b$ & U & 0 & 4 \\
\hline
\end{tabular}

C, carboplatin; Cis, cisplatinum; C\&T, carboplatin and paclitaxel.

previously documented for UPSC ${ }^{(17,19)}$. Thirteen percent of patients had a personal history of breast cancer, identical to that observed by Slomovitz et al. ${ }^{(17)}$ and similar to Barakat et al. ${ }^{4}$ who observed a similar rate of high-risk endometrial subtypes in breast cancer patients regardless of tamoxifen use. The observed high proportion of UPSC in breast cancer patients appears not to be related to BRCA gene status, which confers an increased risk of ovarian papillary serous carcinoma and EEC only ${ }^{(6,7)}$. This suggests that the observed association between UPSC and breast cancer may be due to the presence of mutations in a variety of other cancer predisposing genes such as P53 and cerbB2.

Only $63 \%$ of the biopsies were diagnosed as pure or mixed UPSC histologic patterns, in line with previous reports ${ }^{(9,12,14,17,20-22)}$, which have reported the accuracy of preoperative biopsy from $65 \%$ to $93 \%$. Most biopsies not explicitly stated as UPSC were given grade 3 endometrial carcinoma diagnoses or classified according to the other major histologic subtype, usually CCEC. However, on retrospective review of the biopsies, only one $(1.5 \%)$ of the preoperative biopsies did not contain any UPSC, according to traditional diagnostic criteria, and the major pattern in this case was CCEC. This may be explained by the heterogeneity of the disease.

We sought to evaluate whether $25 \%$ UPSC pattern, the value above which the tumor is classified as UPSC, is prognostically valid ${ }^{(11)}$. We found that the survival of patients with less than $25 \%$ or more than $25 \%$ UPSC in the biopsy or the resection was the same, suggesting that tumors should be classified, and managed, as UPSC regardless of the percentage of UPSC in the pathologic specimen. Our lowest percentage of UPSC used was $5 \%$. In addition, in keeping with previous reports, pure or mixed histologic patterns did not show any survival difference, and survival was the same regardless of whether UPSC was mixed with a highrisk subtype, such as CCEC, or a low-risk subtype, such as $\operatorname{EEC}^{(9,17)}$, suggesting that UPSC is biologically dominant in heterogeneous tumors.

We found that a low number of patients underwent complete surgical staging ( $40 \%$ omental staging, $11.9 \%$ pelvic node clearance, $50 \%$ cytology), which may in part be explained by the changing surgical practice over the period analyzed and difficulties with the interpretation of the diagnostic biopsies. While this raises the possibility that the patients might not be accurately surgically staged, our survival rates are consistent with the gencrally accepted long term survival rates of $35-50 \%$ for patients with stage I and II UPSC and $0-15 \%$ for patients with stage III and IV diseases $^{(23)}$

Gehrig et al. ${ }^{(24)}$ showed that the sensitivity of a visually negative omentum was $89 \%$, concluding that, with a microscopic metastasis rate of $4 \%$, surgical sampling does not need to be included in the routine surgical staging of UPSC. The clinical consequences of understaging is underscored by the fact that in our data two recurrences (13\%) occurred in biopsy positive omenta that were not removed by omentectomy, and these two patients had stage III disease that, while still a poor prognosis group, may have benefited from additional abdominal surgical clearance. Omentectomy may result in better local control of disease and more directed and appropriate adjuvant therapy. Unfortunately, our data were too small to establish a link between omentectomy and survival. Although we had a low rate of pelvic lymphadenectomy, the diagnostic value of systematic lymphadenectomy has recently been validated by Slomovitz et al. ${ }^{(17)}$, who reported $19 \%$ lymph node involvement in patients without myometrial invasion. Furthermore, the importance of performing a formal lymph node dissection is emphasized by the absence of pelvic sidewall failures following observation in a population at risk for recurrence ${ }^{(25)}$ and may explain why two of the patients, who had not underwent formal pelvic lymphadenectomy, had pelvic recurrences.

We did not show any significant impact of adjuvant radiotherapy or chemotherapy on PFS or OS. Ramondetta et al. ${ }^{(26)}$ showed that single-agent paclitaxel showed a tumor response in $77 \%$ of patients and used in a neoadjuvant fashion with cisplatin, and Zanotti et al. ${ }^{(27)}$ observed responses in eight out of nine patients. In addition, Kelly et al. ${ }^{(22)}$ recently showed that platinum-based chemotherapy improved diseasefree survival and OS of patients with stage I UPSC and vaginal cuff radiation provided local control of disease. However, our study failed to show that treatment with these agents converted into an objective improvement in PFS or OS. This is probably due to the short average follow-up time (14 months) and possibly the inadequate surgical staging, resulting in patients being understaged.

Twenty-two percent of patients in this study had further disease. This is far fewer than the percentage 
observed by other groups, who have documented recurrence rates of between $50 \%$ and $75 \%$, using various adjuvant therapies, radiotherapy alone, or with the addition of chemotherapy using platinum and/or paclitaxel alone, as used in our study ${ }^{(27-30)}$. Our results, therefore, compare favorably with similarly staged tumors at equivalent median follow-up, although similar to these studies stage III and IV tumors remain a particular management problem due to higher rates of recurrence $36 \%$ and $33 \%$ for stage III and IV carcinomas compared with $12 \%$ and $14 \%$ for stage I and II carcinomas, respectively). Establishing the exact reasons for favorable recurrence rates is hampered by limited patient numbers and inconsistent and nonrandomized adjuvant therapies, but it appears that surgery produces good regional control in this group, with $67 \%$ locoregional control compared with the $87 \%$ observed by Sood et al. ${ }^{(28)}$.

Despite having been described as a distinct clinical entity for over 20 years, UPSC remains a management challenge for the multidisciplinary team due to the paucity of understanding on the epidemiology, pathogenesis, natural history, and optimal treatment strategies for this important and aggressive variant of endometrial adenocarcinoma. These data contribute to the growing body of literature on UPSC and address diagnostic and treatment uncertainties for the pathology, surgical, and oncologic teams. This study raised awareness for the need of accurate and complete surgical staging at a local level. However, we have not altered our approach to the use of adjuvant chemoradiotherapy for patients with UPSC as our patient numbers were small with a limited follow-up time. This study contributes to the increasing local, national, and international awareness of the need to invest in randomized clinical research trials on UPSC, at a time where alternative treatment modalities may become increasingly effective.

\section{References}

1 Lauchlan SC. Tubal (serous) carcinoma of the endometrium. Arch Pathol Lab Med 1981:105:615-8.

2 Hendrickson M, Ross J, Eifel PJ, Cox RS, Martinez A, Kempson R. Adenocarcinoma of the endometrium: analysis of 256 cases with carcinoma limited to the uterine corpus. Pathology review and analysis of prognostic variables. Gynecol Oncol 1982;13:373-92.

3 Hendrickson M, Ross J, Eifel P, Martinez A, Kempson R. Uterine papillary serous carcinoma: a highly malignant form of endometrial adenocarcinoma. Am / Surg Pathol 1982;6:93-108.

4 Barakat RR, Wong G, Curtin JP, Vlamis V, Hoskins WJ. Tamoxifen use in breast cancer patients who subsequently develop corpus cancer is not associated with a higher incidence of adverse histologic features. Cynecol Oncol 1994:55:164-8.

5 Magriples U, Naftolin F, Schwartz PE, Carcangiu ML. High-grade endometrial carcinoma in tamoxifen-treated breast cancer patients. J Clin Oncol 1993;11:485-90.
6 Geisler JP, Sorosky JI, Duong HL et al. Papillary serous carcinoma of the uterus: increased risk of subsequent or concurrent development of breast carcinoma. Gynecol Oncol 2001;83:501-3.

7 Goshen R, Chu W, Elit L et al. Is uterine papillary serous adenocarcinoma a manifestation of the hereditary breast-ovarian cancer syndrome? Gynecol Oncol 2000;79:477-81.

8 Lavie $\mathrm{O}$, Hornreich $\mathrm{G}$, Ben Arie A et al. BRCA germline mutations in Jewish women with uterine serous papillary carcinoma. Gynecol Oncol 2004;92:521-4.

9 Goff BA, Kato D, Schmidt RA et al. Uterine papillary serous carcinoma: patterns of metastatic spread. Gynecol Oncol 1994;54:264-8.

10 Christman JE, Kapp DS, Hendrickson MR, Howes AE, Ballon SC Therapeutic approaches to uterine papillary serous carcinoma: a preliminary report. Gynecol Oncol 1987;26:228-35.

11 Walker AN, Mills SE. Serous papillary carcinoma of the endometrium. A clinicopathologic study of 11 cases. Diagn Gynecol Obstet 1982;4:261-7

12 Sherman ME, Bitterman P, Rosenshein NB, Delgado G, Kurman RI Uterine serous carcinoma. A morphologically diverse neoplasm with unifying clinicopathologic features. Am / Surg Pathol 1992;16:600-10.

13 Abeler VM, Kjorstad KE. Serous papillary carcinoma of the endometrium: a histopathological study of 22 cases. Gynecol Oncol 1990;39:266-71.

14 Carcangiu ML, Chambers JT. Uterine papillary serous carcinoma: a study on 108 cases with emphasis on the prognostic significance of associated endometrioid carcinoma, absence of invasion, and concomitant ovarian carcinoma. Gynecol Oncol 1992;47:298-305.

15 Chambers JT, Merino M, Kohorn EI, Peschel RE, Schwartz PE. Uterine papillary serous carcinoma. Obstet Gynecol 1987;69:109-13.

16 Christopherson WM, Alberhasky RC, Connelly PJ. Carcinoma of the endometrium. II. Papillary adenocarcinoma: a clinical pathological study, 46 cases. Am J Clin Pathol 1982;77:534-40.

17 Slomovitz BM, Burke TW, Eifel PJ et al. Uterine papillary serous carcinoma (UPSC); a single institution review of 129 cases. Gynecol Oncol 2003;91:463-9.

18 Sykiotis K, Kondi-Pafiti A, Giannaris D, Kouvaris J, Papayannopoulou A, Salamalekis E. Uterine serous papillary carcinoma clinical and immunopathological study of 9 cases. Eur / Gynnecol Oncol 2001; 22:456-8.

19 Dunton CJ, Balsara G, McFarland M, Hernandez E. Uterine papillary serous carcinoma: a review. Obstet Gynecol Suro 1991;46:97-102.

20 Bristow RE, Asrari F, Trimble EL, Montz FJ. Extended surgical staging for uterine papillary serous carcinoma: survival outcome of locoregional (stage I-III) disease. Gynecol Oncol 2001;81:279-86.

21 Tay EH, Ward BG. The treatment of uterine papillary serous carcinoma (UPSC): are we doing the right thing?. Int / Gynecol Cancer 1999;9:463-9.

22 Kelly MG, O'malley DM, Hui $P$ et al. Improved survival in surgical stage I patients with uterine papillary serous carcinoma (UPSC) treated with adjuvant platinum-based chemotherapy. Gynecol Oncol 2005;98:353-9.

23 Nicklin JL, Copeland LJ. Endometrial papillary serous carcinoma: patterns of spread and treatment. Clin Ubstet Ciynecol 1996:39:686-95.

24 Gehrig PA, Van Le L, Fowler WC Jr. The role of omentectomy during the surgical staging of uterine serous carcinoma. Int I Gynecol Cancer 2003;13:212-5.

25 Huh WK, Powell M, Leath CA III et al. Uterine papillary serous carcinoma: comparisons of outcomes in surgical stage I patients with and without adjuvant therapy. Gynecol Oncol 2003;91:470-5.

26 Ramondetta L, Burke TW, Levenback C, Bevers M, Bodurka-Bevers $D$, Gershenson DM. Treatment of uterine papillary serous carcinoma with paclitaxel. Gynecol Oncol 2001:82:156-61.

27 Zanotti KM, Belinson JL, Kennedy AW, Webster KD, Markman M. The use of paclitaxel and platinum-based chemotherapy in uterine papillary serous carcinoma. Gynecol Oncol 1999;74:272-7.

28 Sood BM, Jones J, Gupta S et al. Patterns of failure after the multimodality treatment of uterine papillary serous carcinoma. Int Radiat Oncol Biol Phys 2003;57:208-16.

29 Mallipeddi P, Kapp DS, Teng NN. Long-term survival with adjuvant whole abdominopelvic irradiation for uterine papillary serous carcinoma. Cancer 1993;71:3076-81.

30 Frank AH, Tseng PC, Haffty BG et al. Adjuvant whole-abdominal radiation therapy in uterine papillary serous carcinoma. Cancer $1991 ; 68: 1516-9$.

Accepted for publication November 1, 2005 
Available online at www.sciencedirect.com

ScienceDirect

Gynecologic Oncology xx (2006) xxx-xxx

www,elsevier.com/locate/ygyno

Letter to the Editor

The pattern of CD98 expression is different between uterine serous papillary carcinoma and endometrioid endometrial carcinoma

\section{To the Editor:}

CD98 is a disulphide-linked $125-\mathrm{kDa}$ heterodimeric membrane glycoprotein, which is found on the cell surface of most normal cells, and accumulating evidence suggests that it may have a role in tumour progression and metastasis. We have investigated the expression of CD98 in uterine serous papillary carcinoma (UPSC) and endometrioid endometrial carcinoma (EEC), tumour types we selected for study because of differing pathogeneses and patterns of local invasion and metastasis.

CD98 has been shown to be involved in cellular proliferation, cell transformation, cell fusion, cell adhesion and the Ltype amino acid transporter (LAT) system, in addition to regulating integrin activation, and therefore integrin signalling and anchorage independent growth [1]. Its expression is upregulated in a variety of tumours including those of larynx, where a diffuse pattern of expression is associated with more extensive disease and poorer differentiation [2]. A recent study showed down-regulation of gene expression of CD98 in cell lines derived from metastatic adenoid cystic carcinoma compared to cell lines created from primary adenoid cystic carcinomas [3]. To date, no studies have been published on the pattern of expression of CD98 in endometrial carcinomas.

Endometrial carcinoma is the most common pelvic genital malignancy in the western world. The most common subtype is EEC, which accounts for about $80 \%$ and UPSC accounts for approximately $10 \%$ of endometrial carcinomas. UPSC is associated with lymphovascular invasion and widespread dissemination and has a significantly poorer prognosis at Stages 1,2 and 3 compared to EEC.

This study was undertaken to determine whether there is differential expression of CD98 between EEC and UPSC, which might explain the increased aggressiveness of UPSC compared to
EEC. We examined levels of expression not only in the central viable part, but also at the invasive edge of these tumours.

We created tissue microarrays (TMAs) from 73 cases of UPSC and 20 cases of grade 3 EEC using the established technique [4]. At least three cores were taken from the viable central part and from the invasive edge of the tumours. Immunohistochemistry (IHC) was performed for CD98 (Primary polyclonal IgG antibody, Santa Cruz, dilution 1:200) with tonsil as the positive contru.. Negative controls were obtained by omitting the primary antibody. All slides were stained using the standard streptavidin biotin procedure for IHC. All CD98 expression was membranous and was assessed using a histoscore. This was calculated by multiplying the intensity of staining $(1=$ weak, $2=$ moderate and $3=$ strong $)$ by the percentage of positively stained cells $(1=<20 \%$, $2=20-80 \%$ and $3=>80 \%$ ), thus giving a score between 1 and 9 . To minimise erroneous results, the average score of the cores sampled from each case was used in calculations.

Table 1 shows a summary of the results. Both tumour types showed specific staining of neoplastic cells, but there was significantly greater CD98 expression in EEC compared to UPSC. The invasive edge of UPSC showed significantly greater expression of CD98 compared to the viable central part of $\mathrm{tit}$ tumour, whereas in EEC there was a tendency (which did not achieve significance) for expression to be decreased at the invasive edge.

These findings may reflect the different biological properties of these tumours. EEC usually arises on a background of oestrogen excess, commonly expresses oestrogen receptors (ER), and can produce intratumoural oestrogen using aromatase. In contrast, UPSC usually arises from atrophic endometrium, is generally ER negative and does not produce intratumoural oestrogen. Lessey et al. [5] found that integrin expression correlated with steroid receptor status, as well as with grade, stage and depth of invasion. We found significantly higher ER and progesterone receptors (PR) positivity in our EEC cases compared to UPSC (results not shown).

Our finding of increased expression in the viable central part ${ }^{\circ}$ EEC and a non-significant decrease at the invasive edge is similar to that described by Huang et al. in adenoid cystic carcinoma [3]. In

Table 1

Expression of CD98 in the central part of the tumour and at the invasive edge

\begin{tabular}{|c|c|c|c|c|}
\hline \multirow{2}{*}{$\begin{array}{l}\text { CD98 (Primary polyclonal IgG } \\
\text { antibody, Santa Cruz, dilution } 1: 200 \text { ) }\end{array}$} & \multicolumn{2}{|l|}{ UPSC } & \multicolumn{2}{|l|}{ EEC } \\
\hline & Tumour & Invasive edge & Tumour & Invasive edge \\
\hline & $* 4.32^{\prime}$ & $5.10^{\prime}$ & $* 5.94$ & 3.92 \\
\hline
\end{tabular}

* $P=0.009$ (Mann-Whitney $U$ test).

' $P=0.005$ (Wilcoxon Signed Ranks).

0090-8258/\$ - see front matter (c) 2006 Elsevier Inc. All rights reserved.

doi:10.1016/j.ygyno.2006.09.026 
contrast, lower levels of CD98 were seen in the viable central part of UPSC when compared to the invasive edge. This raises the possibility that $\mathrm{CD} 98$ has different roles in EEC compared to UPSC. The high CD98 in the viable central part of EEC may be due in part to the relative increase in PR and ER, but it is also possible that CD98 plays a role in increasing nutrient uptake in proliferating cells via the LAT system. In contrast, the increased expression of CD98 at the invasive edge of UPSC compared to the viable central part may reflect activation of the $\beta 1$ integrin signalling pathway, increased anchorage independent growth and thus invasion.

We have shown differences in expression of CD98 between EEC and UPSC, which may reflect the different biological properties of these tumours. Further investigation of CD98 interactions with cell adhesion molecules may cast light on mechanisms of tumour invasiveness and metastasis.

\section{References}

[1] Rintoul RC, Buttery RC, Mackinnon AC, et al. Cross-linking CD98 promotes integrin-like signaling and anchorage-independent growth. 117. Mol Biol Cell 2002;13(8):2841-52.
[2] Esteban F, Ruiz-Cabello F, Concha A, et al. Relationship of $4 \mathrm{~F} 2$ antigen with local growth and metastatic potential of squamous cell carcinoma of the larynx. 285. Cancer 1990;66(7):1493-8.

[3] Huang D, Chen W, He R, et al. Different cDNA microarray patterns of gene expression reflecting changes during metastatic progression in adenoid cystic carcinoma. 68. World J Surg Oncol 2003;1(1):28.

[4] Hoos A, Cordon-Cardo C. Tissue microarray profiling of cancer specimens and cell lines: opportunities and limitations. 1. Lab Invest 2001;81 (10): $1331-8$.

[5] Lessey BA, Albelda S, Buck CA, et al. Distribution of integrin cell adhesion molecules in endometrial cancer. 1. Am J Pathol 1995;146 (3):717-26.

Hannah Monaghan* Alistair R.W. Williams Department of Laboratory Services (Pathology), Royal Infirmary of Edinburgh, 51 Little France Crescent, Edinburgh, EHI6 4SA, UK E-mail address: hannah.monaghan@luht.scot.nhs.uk. *Corresponding author. Fax: +440131 2427136.

22 August 2006 


\title{
The role of matrix metalloproteinases $-2,-7$ and -9 and $\beta$-catenin in high grade endometrial carcinoma
}

\author{
H Monaghan, N MacWhinnie \& A R W Williams \\ Department of Laboratory Services (Pathology), Royal Infirmary of Edinburgh, Edinburgh, UK
}

Date of submission 15 March 2006

Accepted for publication 30 May 2006

Monaghan H, MacWhinnie N \& Williams A R W

(2006) Histopathology

\section{The role of matrix metalloproteinases-2, -7 and -9 and $\beta$-catenin in high grade endometrial carcinoma}

Aims: To determine the expression of matrix metalloproteinases (MMPs)-2, -7 and -9 and $\beta$-catenin in uterine serous carcinoma (USC) and endometrioid endometrial carcinoma (EEC) and to investigate any difference in expression between EEC and USC which might explain the mechanism of invasion and aggressive behaviour of USC.

Methods and results: Tissue microarrays were created from the viable central part and from the invasive edge of 20 cases of grade 3 EEC and 73 cases of USC. Immunohistochemistry was performed using antibodies to MMPs-2, -7 and -9 and $\beta$-catenin. MMPs- $2,-7$ and -9 and $\beta$-catenin were present in both tumour types; there was significantly higher expression of MMPs- 2 and -9 in EEC compared with USC and significantly increased expression of MMPs 2 and 9 by carcinoma cells at the invasive edge of USC.

Conclusions: MMPs-2, -7 and -9 and $\beta$-catenin are present in EEC and USC. The increased expression of MMPs -2 and -9 by carcinoma cells at the invasive edge of USC is possibly due to increased binding of MMPs secreted by the stromal cells to carcinoma cells, thus equipping the USC carcinoma cells with proteases for invasion.

Keywords: $\beta$-catenin, endometrial carcinoma, matrix metalloproteinases, uterine serous carcinoma

Abbreviations: BM, basement membrane; ECM, extracellular matrix; EEC, cndometrioid endometrial carcinoma; MMP, matrix metalloproteinase; TMA, tissue microarray; USC, uterine serous carcinoma

\section{Introduction}

Endometrial carcinoma is the most common female genital malignancy in the western world. ${ }^{1}$ There are two main types of endometrial carcinoma: endometrioid endometrial carcinoma (EEC) (Type I) and nonendometrioid endometrial carcinoma (Type II), which mainly consists of uterine serous carcinoma (USC), clear cell carcinoma and malignant mixed mesudermal tumour (carcinosarcoma). EEC accounts for approximately $80 \%$ of all endometrial carcinomas and usually arises from atypical hyperplasia of the endometrium. ${ }^{2-4}$ It is associated with oestrogen excess. USC accounts for

Address for correspondence: Dr Hannah Monaghan. Consultant Pathologist, Department of Laboratory Services (Pathology), Royal Infirmary of Edinburgh, 51 Little France Crescent, Edinburgh EH16 4SA. UK. e-mail: hannah.monaghan@luht.scot.nhs.uk approximately $10 \%$ of cases of endometrial carcinoma ${ }^{2}$ and makes up the majority of the Type II carcinomas. It is an aggressive tumour, usually occurring in elderly women and is thought to arise from endometrial intraepithelial carcinoma. ${ }^{5-7}$ It is not associated with either atypical hyperplasia or a hyperoestrogenic state. Although USC comprises $10 \%$ of cases of endometrial carcinoma, ${ }^{2}$ it accounts for a much smaller proportion of Stage I endometrial carcinomas. ${ }^{8-10}$ It is upstaged at the time of surgery in $60 \%$ of cases. ${ }^{11-15}$ Hui et al. ${ }^{16}$ examined a series of 40 cases of minimal uterine serous carcinoma. Nine of these were endometrial intraepithelial carcinoma and 31 were cases of Stage 1a USC. They demonstrated that only when the carcinoma was confined to an endometrial polyp did the patients have good prognosis. This aggressive behaviour raises the possibility that USC interacts with the extracellular matrix $(\mathrm{ECM})$ in a different way from $\mathrm{EEC}$.

(ㄷ) 2006 The Authors. Journal compilation @ 2006 Blackwell Publishing Limited.

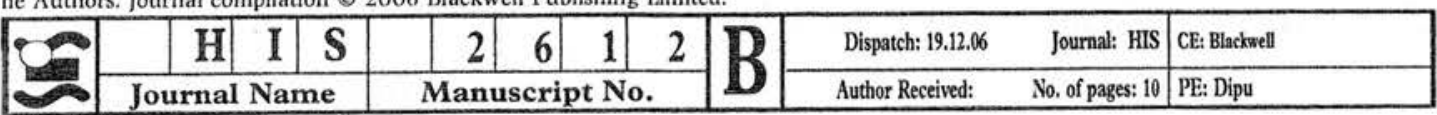


Invasion and metastasis of tumour cells requires a variety of complex interdctiuns including cell-ccll interactions, cell-matrix interactions and degradation of the ECM. Basement membranes (BMs) constitute the major natural barrier that cancer cells must cross several times during the metastatic cascade. Liotta et al. ${ }^{17}$ have suggested a hypothetical model in which tumour invasion and metastasis results from repetition of three steps: adhesion of cancer cells to BM glycoproteins; degradation of BMs by speciflc proteolytic emzymes; and migration of cancer cells.

The theory of tumour progression and heterogeneity is well established. Tumours are known to lose clonality early in development and generate subclones with varying characteristics resulting from mutations. In theory, only the selected subclone will possess the appropriate genetic characteristics in order to invade and metastasize.

Matrix metalloproteinases (MMPs), a family of zincdependent endoproteinases, are widely accepted to play a role in the invasion and metastasis of tumours. ${ }^{18.19}$ Under normal physiological conditions, MMPs are expressed at a very low level in adult tissues, except in tissues that undergo remodelling such as cycling endometrium, normal breast tissue at the time of involution and the skin during wound healing. ${ }^{18.20-22}$ Increased MMP expression and proteolytic degradation of ECM have been detected in a wide range of cancers, including those arising from the breast, colon, ovary and lung and MMP expression has been correlated with primary tumour growth and angiogenesis and also tumour invasion and metastasis. ${ }^{18,19,23-27}$

Although some MMPs are synthesized and secreted by cancer cells, others are synthesized and secreted by stromal cells and then bind to the cell membrane of tumour cells. ${ }^{28-34}$ MMP expression appears to vary among host organ microenvironments and stromal MMPs may promote metastasis to one organ in preference to another. Therefore, antimetastatic effects based on MMP inhibition may be dependent on MMPs derived from specific organ microenvironments as well as tumour cells. ${ }^{35}$

ProMMP-2, the precursor to active MMP-2, is widely expressed in normal tissue and the MMP-2 gene has been described as a 'house-keeping' gene for its role in normal cellular processes. ${ }^{36}$ In contrast, active MMP-2 is increased significantly in some neoplastic tissues and absent in most normal tissues. MMP-2 contributes to cell migration by a mechanism involving interaction with collagen. ${ }^{37}$ MMP-2 has been demonstrated in endometrial carcinoma cells. ${ }^{38}$
Altered expression of MMP-7 has been demonstrated in EEC and increased expression of MMP 7 is associated with lymph node metastasis. ${ }^{38-41}$ Matrisian et al. ${ }^{36}$ have shown that induction of MMP-7 expression in tumour cells increases tumorigenicity and metastases, while inhibition of MMP-7 leads to decreased metastasis. ${ }^{42.43}$ In addition, they found that MMP-7 expression appeared to be associated with a less aggressive phenotype, despite being involved in invasion. In contrast, Misugi et al. ${ }^{38}$ have demonstrated that increased cxpression of MMP 7 is found in high-grade endometrial carcinomas.

$\beta$-Catenin has roles in both cell-cell adhesion and intracellular signalling. It links to intracellular E-cadherin and thus connects E-cadherin to the actin cytoskeleton. ${ }^{44-46}$ Cadherin-catenin complexes also have roles in intercellular communication and modulation of cell function in both normal and malignant tissues. Failure to assemble the E-cadherin-catenin complex or to connect properly to the actin cytoskeleton results in loss of cell adhesion, which may be involved in tumour spread. Signalling through the cadherin-catenin complexes is involved in the regulation of epidermal growth factor receptor distribution. $^{47}$

MMP-9 expression has been shown to be altered in EEC and increased expression is associated with myometrial invasion. ${ }^{38-41}$ In addition, Park et al. ${ }^{48}$ have shown that synthesis and secretion of MMP-9 is down-regulated by $\beta$-oestradiol. Cioppi et al. ${ }^{49}$ found that MMP-9 expression is higher in oestrogen receptorpositive endometrial carcinomas.

Although USC comprises only $10 \%$ of endometrial carcinomas, it accounts for a greater proportion of deaths. This study was undertaken to investigate the expression of MMPs-2, -7 and -9 in USC and EEC. and in particular to see if there was any difference in expression between the two tumour types which might explain the observed difference in aggressive behaviour. As $\beta$-catenin has a role in cell-cell adhesion and studies on colon and breast carcinoma have shown both positive and negative correlations between the expression of $\beta$-catenin and MMP-7, we also examined the expression of $\beta$-catenin in these tumours. It is well recognized that tumours are heterogeneous and examining the central part of the tumour alone may give misleading results as regards the invasiveness of the tumour as a whole. We used tissue microarrays (TMAs) to examine a relatively large number of cases, in order to be able to demonstrate differences in expression of these proteins between the viable central part of the tumour and the invasive edge in USC and EEC. 
The Medicine/Clinical Oncology II Research Ethics Committee of the Lothian Research Ethics Committee gave ethical approval for this study.

\section{Materials and methods}

\section{TISSUES}

Archival tissue samples were selected from the Royal Infirmary of Edinburgh Department of Pathology, the slides were reviewed by H.M. and A.R.W.W. the diagnosis of USC or EEC was confirmed and appropriate blocks selected. Haematoxylin and eosin (H\&E) sections then had at least one area from the centre of the tumour and invasive edge marked on the slide by the pathologist. The samples included 20 International Federation of Gynaecology and Obstetrics (FIGO) Grade 3 cases of EBC and 73 cases of USC. As we did not identify any cases of endometrial intraepithelial carcinoma and only three cases of USC were at Stage 1a, these cases were not treated as a separate group. All tissue had been routinely fixed in $4 \%$ buffered formaldehyde and processed to paraffin blocks by routine methods.

\section{TISSUE MICROARRAY}

A microarray instrument (Beecher Instruments, Sun Prairie. WI, USA) was used. In order to construct the microarray, empty paraffin blocks of a depth of 5$10 \mathrm{~mm}$ were produced. Cores of wax, $0.8 \mathrm{~mm}$ diameter, were extracted from the empty blocks and replaced with cores of $0.6 \mathrm{~mm}$ diameter taken from the tissue blocks at sites corresponding to the previously selected areas on the H\&E slides. Three cores were taken from each area marked on the slide, so that at least three cores were taken from each case. The cores were punched at 1-mm intervals, at least three cores per case, to decrease the risk of aberrant results due to tumour heterogeneity. A grid system with each core having a coordinate reference ( $\mathrm{X}$ axis, $\mathrm{X}$ axis) was used to allow cross reference between core location and parent case. The microarray blocks were identified as EEC tumour, EEC invasive edge or USC tumour or USC invasive edge.

Once the microarrays were complete, the blocks were sealed in a $60^{\circ} \mathrm{C}$ oven for $10 \mathrm{~min}$.

\section{IMMUNOHISTOCHEMISTRY}

From the array blocks, sections were cut at $3 \mu \mathrm{m}$ thickness and mounted on positively charged capillary action slides (Dako, Copenhagen, Denmark) and incu- bated at $60^{\circ} \mathrm{C}$ overnight. The slides were dewaxed and rehydrated prior to antigen retrieval.

\section{MMP-2}

Antigen retrieval was performed by immersing the test slides along with a section of normal appendix (as the positive control) in $0.01 \mathrm{M}$ ethylenediamine tetraaceticacid (EDTA), pH 8.0 and microwaving at high power for $15 \mathrm{~min}$. The primary monoclonal antibody was the 17B11 clone (NovoCastra Laboratories, Newcastle, UK) and was used at a dilution of $1: 40$. Negative controls were obtained by omitting the primary antibody.

\section{MMP -7}

No antigen retrieval was performed. A slide of breast carcinoma was used as the positive control. The primary monoclonal antibody was clone 1D2 (Chemicon International, Chandlers Ford, UK) and was used at a dilution of $1: 25$. Negative controls were obtained by omitting the primary antibody.

\section{MMP-9}

Antigen retrieval was performed by immersing the test slides along with a slide of kidney (as the positive control) in $0.01 \mathrm{~m}$ EDTA, pH 8.0 and pressure cooking for $7 \mathrm{~min}$. The primary monoclonal antibody was clone 15W2 (NovoCastra Laboratories) and was used at a dilution of $1: 80$. Negative controls were obtained by omitting the primary antibody.

\section{$\beta$-CATENIN}

Antigen retrieval was performed by immersing the test slides along with a slide of tonsil (as the positive control) in $0.01 \mathrm{M}$ EDTA, pH 8.0 and microwaving at high power for $15 \mathrm{~min}$. The primary monoclonal antibody was the $\beta$-catenin-1 clone (Dako) and was used at a dilution of $1: 50$. Negative controls were obtained by omitting the primary antibody.

All slides were stained on the Dako TechMate ${ }^{\mathrm{TM}} 500$ plus, using the Dako ChemMate ${ }^{\mathrm{TM}}$ EnVision ${ }^{\mathrm{TM}}$ Detection kit and the standard operating procedure for routine immunohistochemistry.

\section{SCORING}

The scores for MMP-2. MMP-7, MMP-9 and $\beta$-catenin were calculated by multiplying the intensity of staining (1. weak; 2 , moderate; and 3, strong) by the percentage 
of cells showing positive nuclear staining $(1,<20 \%$; 2, $20-80 \%$; and $3,>80 \%$ ), thus giving a score between 1 and 9 .

\section{STATISTICAL ANALYSIS}

The mean scores of the three areas of tumour assessed for each case of USC and EEC were calculated. The results from the main part of the tumour were used in calculations to determine if there were any differences between tumour types. A paired $t$-test was performed for the parametric results and a Mann-Whitney U-test was performed for the non-parametric results. A $P$-value of $<0.05$ was considered to be significant.

The Wilcoxon matched-pairs signed-ranks test was used to estimate the relationship between staining patterns of the different antibodies used between the main part of the tumour and at the invasive edge. A $P$-value of $<0.05$ was considered to be significant. The Pearson pairwise correlation coefficient was used to determine any correlation between expression of different MMPs and $\beta$-catenin.

\section{Results}

\section{INTERTUMOUR VARIABILITY}

Significantly greater MMP-2 expression was present in EEC carcinoma cells compared with USC carcinoma cells $(P<0.05$, Mann-Whitney $U$-test; Table 1$)$. There was no significant difference in MMP-2 expression between USC and EEC stromal cells. In addition, there was no significant difference in either carcinoma cell or

Table 1. Expression of matrix metalloproteinases (MMPs)-2, -7 and -9 and $\beta$-catenin in the central part of the tumour in uterine serous carcinoma (USC) and endometrioid endometrial carcinoma (EEC)

\begin{tabular}{lll}
\hline Antibody & USC & EEC \\
\hline MMP-2 & $0.53^{*}$ & $1.43^{*}$ \\
\hline MMP-2 stroma & 1.68 & 1.36 \\
\hline MMP-7 & 1.81 & 1.77 \\
\hline MMP-7 stroma & 1.28 & 1.51 \\
\hline MMP-9 & 0.90 & 1.17 \\
\hline MMP-9 stroma & $1.24^{* *}$ & $2.44^{* *}$ \\
\hline$\beta-$ Catenin & 7.05 & 7.78 \\
\hline
\end{tabular}

${ }^{*} P<0.05$ (Mann-Whitney $U$-test); ${ }^{*} P=0.001$ (MannWhitney $U$-test). stromal cell expression of MMP-7 between USC and EEC. Although there was no significant difference in MMP-9 expression between USC and EEC carcinoma cells, there was significantly greater MMP-9 expression seen in EEC stromal cells $(P=0.001$. Mann-Whitney $U$-test; Table 1). There was no significant difference in $\beta$-catenin expression between USC and EEC. Scatterplots and Pearson pairwise correlation coefficient showed no direct or inverse correlation between $\beta$-catenin expression and MMPs- $2,-7$ or -9 .

\section{INTRATUMOUR VARIABILITY}

\section{Uterine serous carcinoma}

There was significantly greater MMP-2 expression in carcinoma cells at the invasive edge compared with those in the viable central part of the tumour $(P=$ 0.045 . Wilcoxon signed-ranks test). However, there was no significant difference in expression of MMP-2 in stromal cells between the invasive edge and the viable central part of the tumour (Table 2). MMP-2 immunoreactivity was present in both the cytoplasm and at the cell membrane (Figure 1B).

There was no significant difference in either MMP-7 or MMP-9 expression betwecn the viable central part of the tumour or the invasive edge in either carcinoma or stromal cells (Table 2). Immunoreactivity of both MMP-7 and MMP-9 in both carcinoma and stromal cells was cytoplasmic and membranous (Figures 1D and $2 B$, respectively).

Table 2. Expression of matrix metalloproteinases (MMPs)-2, -7 and -9 and $\beta$-catenin in the central part of the tumour and at the invasive edge

\begin{tabular}{llllll}
\hline & USC & & & EEC \\
\cline { 6 - 6 } Antibody & Tumour & $\begin{array}{l}\text { Invasive } \\
\text { edge }\end{array}$ & & Tumour & $\begin{array}{l}\text { Invasive } \\
\text { edge }\end{array}$ \\
\hline MMP-2 & 0.53 & $0.79^{*}$ & & 1.43 & 1.69 \\
\hline MMP-2 stroma & 1.68 & 1.54 & 1.42 & 1.46 \\
\hline MMP-7 & 1.81 & 1.69 & 1.77 & 1.61 \\
\hline MMP-7 stroma & 1.27 & 1.21 & 1.52 & 1.51 \\
\hline MMP-9 & 0.90 & 1.08 & 1.14 & 1.09 \\
\hline MMP-9 stroma & 1.24 & 1.22 & 2.51 & 1.76 \\
\hline
\end{tabular}

${ }^{*} P=0.045$ (Wilcoxon signed-ranks test); ${ }^{*} P=0.03$ (Wilcoxon signed-ranks test).

USC, Uterine serous carcinoma; EEC, endometrioid endometrial carcinoma. 

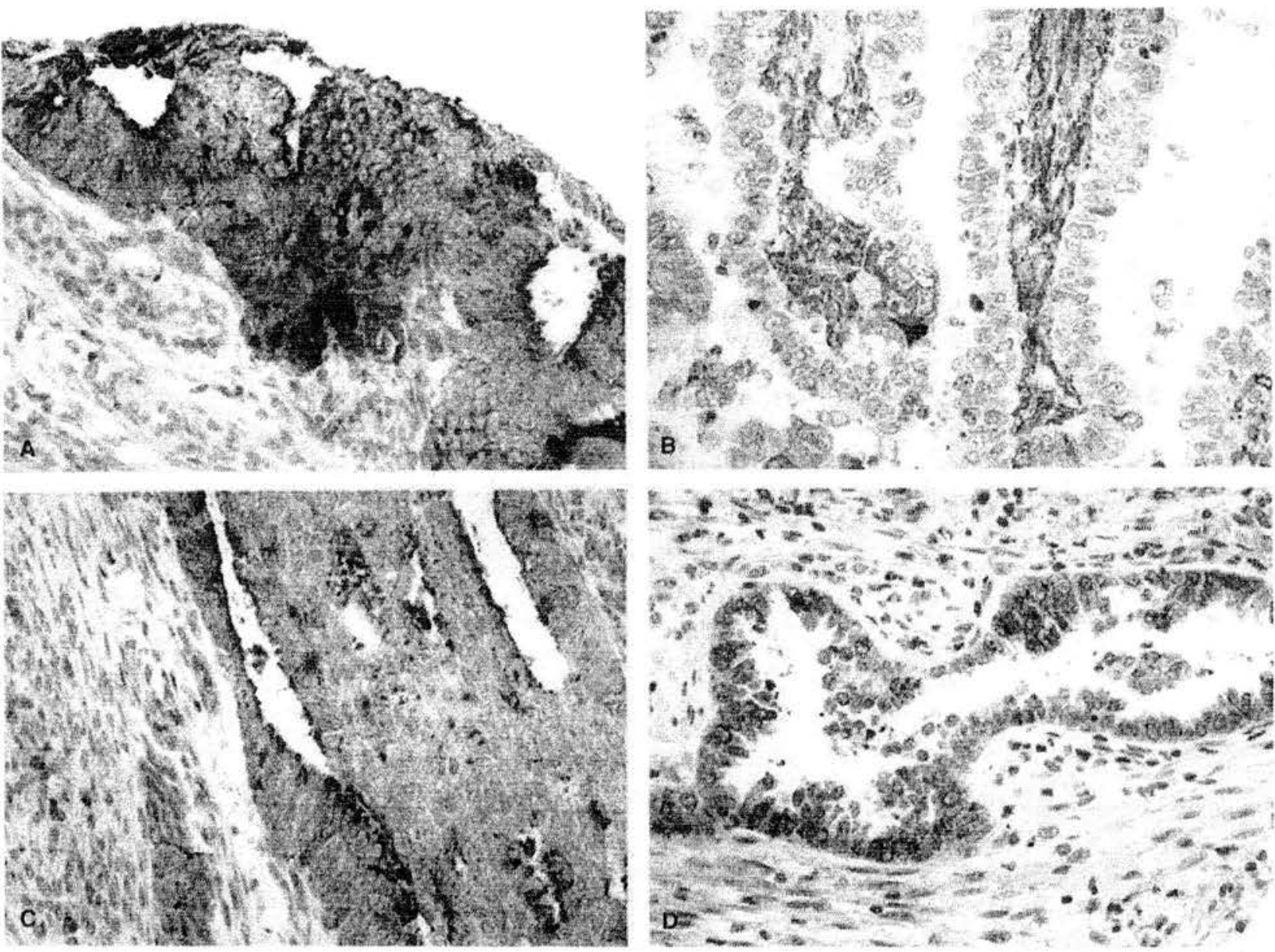

Figure 1. Expresston of matrix metalloproteinases (MMPs). A, MMP-2 in endometrioid endometrial carcinoma (EEC). B, MMP-2 in uterine serous carcinoma (USC), C, MMP-7 in EEC. D, MMP-7 in USC.

There was significantly greater expression of $\beta$-catenin in carcinoma cells at the invasive edge compared with those in the viable central part of the tumour $(P=0.03$, Wilcoxon signed-ranks test, Table 2). However, all $\beta$-catenin expression was membranous (Figure 2D).

There was significantly greater expression of MMP-2 in stromal cells compared with carcinoma cells in both the viable central part of the tumour and at the invasive edge $(P<0.05$ and $P=0.001$, respectively, Wilcoxon signed-ranks test. Table 3). In contrast, there was significantly less expression of MMP-7 in the stromal cells compared with the carcinoma cells in both the viable central part of the tumour and at the invasive edge $(P<0.05$ and $P=0.001$, respectively, Wilcoxon signed-ranks test, Table 3 ). There was significantly less MMP-9 expression in carcinoma cells compared with stromal cells in the viable central part of the tumour $(P=0.02$. Wilcoxon signed-ranks test, Table 3), but there was no significant difference in MMP-9 expression between these cells at the invasive edge.

\section{Endometrioid endometrial carcinoma}

No significant differences in expression of any of the proteins (MMPs-2, $-7,-9$ or $\beta$-catenin) were noted in either carcinoma cells or stromal cells at the invasive edge compared with those in the viable central part of the tumour (Table 2). Expression of all the MMPs was both cytoplasmic and membranous (Figures 1A,C and $2 \mathrm{~A}$, respectively). Two of the $20 \mathrm{EEC}$ cases showed nuclear $\beta$-catenin expression (Figure $2 \mathrm{C}$ ). The remainder showed membranous staining.

There was no significant difference in expression of MMP- 2 between carcinoma and stromal cells in either the viable central part of the tumour or at the invasive 
6 H Monaghan et al.
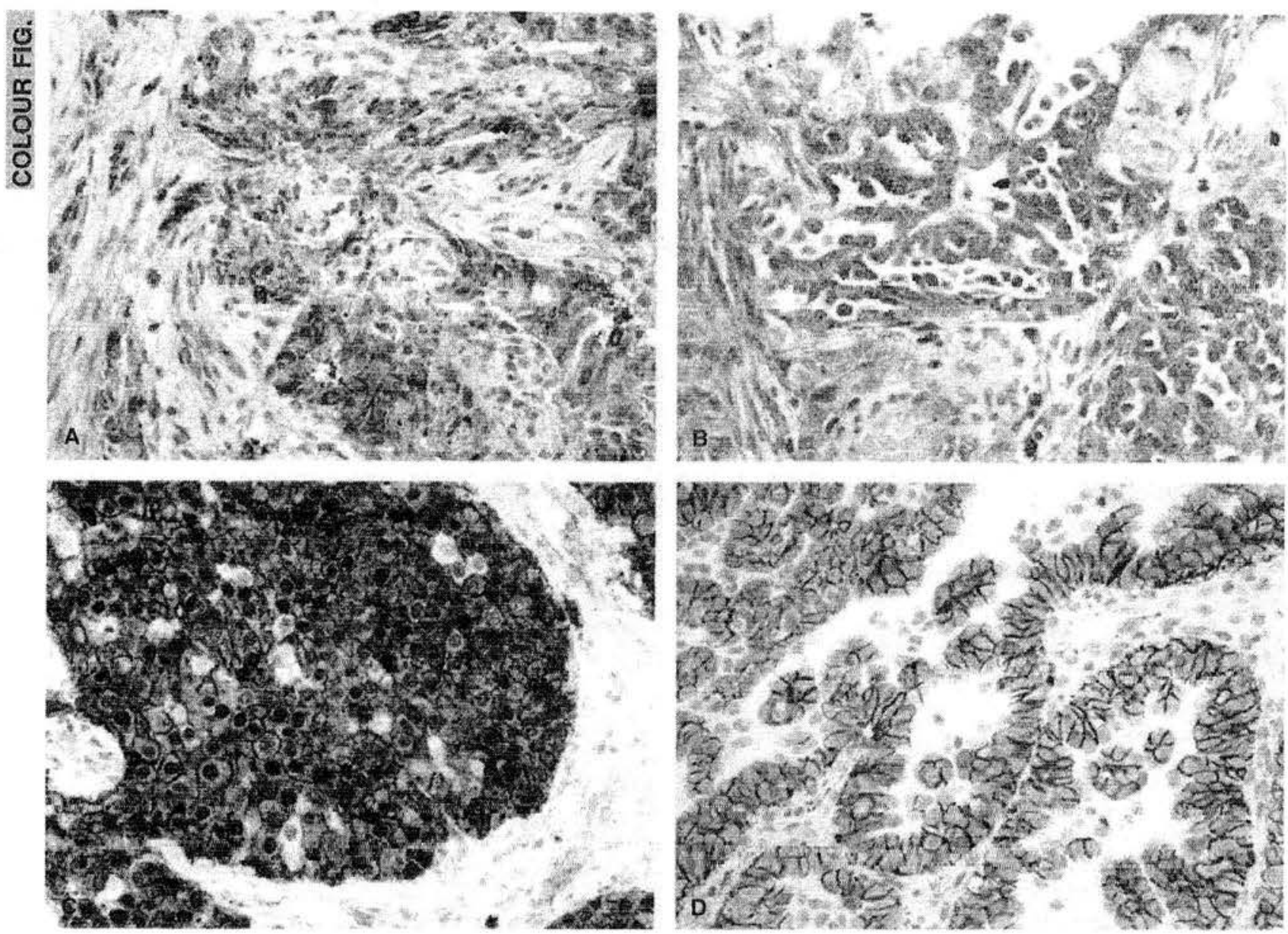

Figure 2. Expression of matrix metalloproteinases (MMPs). A, MMP-9 in endometrioid endometrial carcinoma (EEC). B, MMP-9 in uterine serous carcinoma (USC). C, Nuclear $\beta$-catenin in EEC. D, Membranous $\beta$-catenin in USC.

Table 3. Variation in matrix metalloproteinase (MMP) expression between tumour and stromal cells in uterine serous carcinoma

\begin{tabular}{llllll}
\hline & \multicolumn{2}{l}{ Main tumour } & & \multicolumn{2}{l}{ Invasive edge } \\
\cline { 2 - 3 } \cline { 6 - 7 } Antibody & $\begin{array}{l}\text { Tumour } \\
\text { cells }\end{array}$ & $\begin{array}{l}\text { Stromal } \\
\text { cells }\end{array}$ & & $\begin{array}{l}\text { Tumour } \\
\text { cells }\end{array}$ & $\begin{array}{l}\text { Stromal } \\
\text { cells }\end{array}$ \\
\hline MMP-2 & $0.53^{*}$ & $1.68^{*}$ & & $0.79 t$ & $1.54 \dagger$ \\
\hline MMP-7 & $1.81^{* *}$ & $1.27^{* *}$ & & $1.69+\dagger$ & $1.21+\dagger$ \\
\hline MMP-9 & $0.90^{* * *}$ & $1.24^{* * *}$ & 1.08 & 1.22 \\
\hline
\end{tabular}

${ }^{*} P<0.05$ (Wilcoxon signed-ranks test); ${ }^{*} P<0.05$ (Wilcoxon signed-ranks test); $* * * P=0.02$ (Wilcoxon signedranks test).

$+P=0.001$ (Wilcoxon signed-ranks test); $++P=0.001$ (Wilcoxon signed-ranks test).
Table 4. Variation in matrix metalloproteinase (MMP) expression between tumour and stromal cells in endometrioid endometrial carcinoma

\begin{tabular}{clllll}
\hline & \multicolumn{2}{l}{ Main tumour } & & \multicolumn{2}{l}{ Invasive edge } \\
\cline { 2 - 3 } \cline { 6 - 6 } Antibody & $\begin{array}{l}\text { Tumour } \\
\text { cells }\end{array}$ & $\begin{array}{l}\text { Stromal } \\
\text { cells }\end{array}$ & & $\begin{array}{l}\text { Tumour } \\
\text { cells }\end{array}$ & $\begin{array}{l}\text { Stromal } \\
\text { cells }\end{array}$ \\
\hline MMP-2 & 1.43 & 1.42 & & 1.69 & 1.46 \\
\hline MMP-7 & $1.77^{*}$ & $1.52^{*}$ & & 1.61 & 1.51 \\
\hline MMP-9 & $1.14^{* *}$ & $2.51^{* *}$ & & $1.09+$ & $1.76+$ \\
\hline
\end{tabular}

${ }^{*} P=0.018$ (Wilcoxon signed-ranks test); ${ }^{*} P=0.011$ (Wilcoxon signed-ranks test).

$+P=0.012$ (Wilcoxon signed-ranks test). 
edge (Table 4). There was significantly greater expression of MMP-7 in carcinoma cells compared with stromal cells in the viable central part of the tumour $(P=0.018$. Wilcoxon signed-ranks test, Table 4$)$, but there was no significant difference in expression between the cell types at the invasive edge. Significantly greater expression of MMP-9 was noted in stromal cells compared with carcinoma cells in both the viable central part of the tumour and at the invasive edge $(P=0.011$ and $P=0.012$, respectively, Wilcoxon signed-ranks test, Table 4).

\section{Discussion}

USC is an aggressive endometrial tumour with a notable propensity for lymphovascular space invasion and a poor prognosis. We performed this study to determine whether MMPs have a role in their increased invasiveness in comparison with EEC. We confirmed the findings of Misugi et al. ${ }^{38}$ and Moreno-Bueno et al. ${ }^{50}$ and demonstrated MMPs-2, -7 and -9 and $\beta$-catenin to be present in both USC and EEC.

In addition, the present study looked for differences in expression of these proteins between the different histological tumour types and within the tumours (the viable central part of the tumour and at the invasive edge). We demonstrated MMP-2 expression in both EEC and USC carcinoma cells, confirming the findings of Park et al. ${ }^{48}$ Park et al. ${ }^{48}$ demonstrated that synthesis and secretion of stromal MMP-2 is up-regulated by $\beta$-oestradiol. In addition, they showed that the invasiveness of cells from an endometrial adenocarcinoma cell line was enhanced by recruitment of MMP-2 secreted by endometrial stromal cells to their cell membrane, which was further enhanced by the presence of $\beta$-oestradiol.

The finding of significantly higher MMP-2 expression in EEC carcinoma cells compared with those in USC may partly reflect the different biological properties of these tumours. EEC is a tumour which usually arises on a background of oestrogen excess, which commonly expresses oestrogen receptor and can produce endogenous oestrogen using aromatase. ${ }^{51}$ On the other hand, USC usually arises from atrophic endometrium. is generally oestrogen receptor-negative and does not produce endogenous oestrogen. Park et al. ${ }^{48}$ have demonstrated that $\beta$-oestradiol stimulates MMP-2 secretion in adenocarcinoma cell lines; it is therefore possible that the differential secretion may be due to increased endogenous oestrogen in EEC compared with USC. We found significantly higher oestrogen receptor positivity in our EEC cases compared with USC (results not shown).
We found similar levels of MMP-2 in EEC carcinoma and stromal cells and although there was increased expression of MMP-2 in carcinoma cells at the invasive edge of the tumour, this was not statistically significant. However, we did find a significantly higher level of MMP-2 in USC stromal cells compared with carcinoma cells both in the viable central part of the tumour and at the invasive edge. In addition, the USC carcinoma cells showed a significant increase in MMP-2 expression at the invasive edge compared with the viable central part of the tumour. MMP- 2 is synthesized and secreted by stromal cells and then binds to the TIMP-2:MT-MMP complex on the carcinoma cell surface; ${ }^{32}$ this difference in expression raises the possibility that an invasive subclone of USC uses increased carcinoma cell binding of MMP-2 to equip the cells with the proteases required for invasion.

We did not demonstrate any intertumoral or intratumoral differences in expression of MMP-7. There was significantly more expression of MMP-7 in carcinoma cells compared with stromal cells in both USC and EEC. Our findings are in keeping with the published literature that MMP-7 is generally expressed in tumours. $^{38-41}$ As this study examined high-grade endometrial cancers only, we could not confirm or refute the findings of Misugi et al., ${ }^{38}$ who showed differential expression of MMP-7 between the different grades of EEC.

There was no significant difference in $\beta$-catenin expression between EEC and USC. However, we did find that $\beta$-catenin expression was increased significantly at the invasive edge of USC. All USC cases demonstrated membranous expression of $\beta$-catenin and only two EEC cases $(10 \%)$ demonstrated nuclear expression. All other cases showed membranous expression. Our finding that $10 \%$ of EEC cases showed nuclear expression is less than that reported by Moreno-Bueno et al., ${ }^{52}$ who found that nuclear $\beta$-catenin expression was present in $31 \%$ of EECs and $3 \%$ of non-endometrioid endometrial carcinomas. ${ }^{53}$ It is possible that this discrepancy is due to our case selection of only high-grade EEC, and $\beta$-catenin mutations may be more important in the development of Grade 1 and Grade 2 EEC.

As well as roles associated with cadherin function. $\beta$-catenin is also involved in intracellular signalling, as it is a member of the Wnt pathway. The activation of the Wnt signalling pathway due to $\beta$-catenin mutations has been implicated in the development of some endometrial carcinomas. However, up to $25 \%$ of endometrial carcinomas have $\beta$-catenin nuclear accumulation without evidence of $\beta$-catenin mutations, 
suggesting alterations in other molecules that can modulate the Wnt pathway such as APC, $\gamma$-catenin, AXIN1 and AXIN2. In ovarian cancer the presence of nuclear $\beta$-catenin is an indicator of good prognosis, but whether this has the same effect in endometrial cancers is as yet unknown.

Crawford et al. ${ }^{54}$ have reported a positive correlation between nuclear $\beta$-catenin protein levels and MMP-7 transcripts in colonic carcinoma. They did not find any MMP-7 expression in cells that lacked $\beta$-catenin protein accumulation. Mylona et al. ${ }^{55}$ have demonstrated an inverse correlation with nuclear $\beta$-catenin expression in breast carcinoma. We did not demonstrate any inverse or direct correlation between $\beta$-catenin expression and any of the MMPs. This may be due to the relatively small numbers of cases involved. Alternatively, this may be because the studies that have demonstrated either inverse or direct correlations have focused on colonic carcinoma where $\beta$-catenin expression is nuclear, reflecting mutation in the $\beta$-catenin gene, and the studies by Mylona et al..$^{55}$ in breast carcinoma also focused on $\beta$-catenin mutation.

We found significantly higher expression of MMP-9 in EEC compared with USC and also significantly higher expression of MMP-9 in stromal cells compared with carcinoma cells in the viable central part of the tumour in both EEC and USC. In addition, there was significantly higher expression of MMP-9 in stromal cells compared with carcinoma cells at the invasive edge of the EEC cases. However, there was no significant difference in MMP-9 expression between the invasive edge stromal cells and carcinoma cells in USC. MMP-9 is normally synthesized and secreted by stromal cells and binds to CD44 on carcinoma cells. ${ }^{32}$ The loss of this differential expression between carcinoma cells and stromal cells at the invasive edge of USC cases may indicate that MMP-9 synthesis and secretion by stromal cells is unchanged at the invasive edge of the tumour, but binding of MMP-9 by the invading carcinoma cells is increased, perhaps enhancing their invasive potential.

Hui et al. ${ }^{16}$ have recently demonstrated differences in outcome between patients with Stage 1a USC arising in a polyp and in the 'normal' endometrium. In view of our results showing differences in expression of MMPs- 2 and -9 between the main part of the tumour and the invasive edge. it would be interesting to examine more closely cases of endometrial intraepithelial carcinoma and Stage 1a USC.

In summary, this immunohistochemical study has confirmed that MMPs-,- 7 and -9 and $\beta$-catenin are present in endometrial carcinomas and specifically in both EEC and USC. We found significantly higher expression of MMPs-2 and -9 in EEC compared with USC. This may be due to the known relationship between these MMPs and oestrogen status. Despite the lower expression of MMP-2 and MMP-9 in USC compared with EEC, we did show increased expression of MMP-2 and MMP-9 by carcinoma cells at the invasive edge of USC. This is probably due to increased binding to carcinoma cells of MMPs secreted by the stromal cells. This difference in staining pattern between the viable central part of the tumour and the invasive edge supports the theory of an invasive subclone of USC acquiring activated proteases in order to invade.

\section{References}

1. Creasman WT, Odicino F, Maisonneuve P et al. Carcinoma of the corpus uteri. Int. J. Gynaecol. Obstet. 2003; 83 (Suppl. 1); 79118.

2. Burton JL, Wells M. Recent advances in the histopathology and molecular pathology of carcinoma of the endometrium. Histopathology 1998; 33; 297-303.

3. Santin AD, Bellone S, Gokden M et al. Overexpression of HER$2 /$ neu in uterine serous papillary cancer. Clin. Cancer Res. 2002; 8; $1271-1279$.

4. Santin AD. HER2/neu overexpression: has the Achilles' heel of uterine serous papillary carcinoma been exposed? Gynecol. Oncol. 2003; 88; 263-265.

5. Wheeler DT, Bell KA, Kurman RJ, Sherman ME. Minimal uterine serous carcinoma: diagnosis and clinicopathologic correlation. Am. J. Surg. Pathol. 2000; 24; 797-806.

6. Ambros RA, Sherman ME, Zahn CM, Bitterman P, Kurman RJ. Endometrial intraepithelial carcinoma: a distinctive lesion specifically associated with tumors displaying serous differentiation. Hum. Pathol. 1995; 26; 1260-1267.

7. Spiegel GW. Endometrial carcinoma in situ in postmenopausal women. Am. J. Surg. Pathol. 1995; 19; 417-432.

8. Hendrickson MR, Ross JC, Kempson RL. Toward the development of morphologic criteria for well-differentiated adenocarcinoma of the endometrium. Am. J. Surg. Pathol. 1983; 7; 819-838.

9. Bancher-Todesca D, Neunteufel W, Williams KE et al. Influence of postoperative treatment on survival in patients with uterine papillary serous carcinoma. Gynecol. Oncol. 1998; 71; 344-347.

10. Dembo AJ. The sequential multiple modality treatment of ovarian cancer. Radiother. Oncol. 1985; 3; 187-192.

11. Gehrig PA, Groben PA, Fowler WC Jr, Walton LA, Van Le L. Noninvasive papillary serous carcinoma of the endometrium. Obstet. Gynecol. 2001; 97; 153-157.

12. Jeffrey JF, Krepart GV, Lotocki RJ. Papillary serous adenocarcinoma of the endometrium. Obstet. Gynecol. 1986; 67; 670-674.

13. Kato DT. Ferry JA, Goodman A et al. Uterine papillary serous carcinoma (UPSC): a clinicopathologic study of 30 cases. Gynecol. Oncol. 1995; 59; 384-389.

14. Goff BA, Kato D. Schmidt RA et al. Uterine papillary serous carcinoma: patterns of metastatic spread. Gynecol. Oncol. 1994; 54; 264-268.

15. Dunton CJ, Balsara G, McFarland M, Hernandez E. Uterine papillary serous carcinoma: a review. Obstet. Gynecol. Surv. 1991: 46; 97-102. 
16. Hui P, Kelly M, O'Malley DM. Tavassoli F. Schwartz PE. Minimal uterine serous carcinoma: a clinicopathological study of 40 cases. Mod. Pathol. 2005; 18; 75-82.

17. Liotta LA. Tumor invasion and metastases-role of the extracellular matrix: Rhoads Memorial Award lecture. Cancer Res. 1986; 46; 1-7.

18. Chang C. Werb Z. The many faces of metalloproteases: cell growth, invasion, angiogenesis and metastasis. Trends Cell Biol. 2001: 11: S37-S43.

19. Vihinen P. Kahari VM. Matrix metalloproteinases in cancer: prognostic markers and therapeutic targets. Int. J. Cancer 2002; 99; $157-166$.

20. Parks WC. Matrix metalloproteinases in repair. Wound Repair Regen. 1999; 7; 423-432.

21. Rodgers WH, Osteen KG, Matrisian LM, Navre M, Giudice LC. Gorstein F. Expression and localization of matrilysin, a matrix metalloproteinase, in human endometrium during the reproductive cycle. Am. J. Obstet. Gynecol. 1993; 168 (1 Part 1); 253-260.

22. Rodgers WH. Matrisian LM, Giudice LC et al. Patterns of matrix metalloproteinase expression in cycling endometrium imply differential functions and regulation by steroid hormones. J. Clin. Invest. 1994: 94; 946-953.

23. Stamenkovic I. Matrix metalloproteinases in tumor invasion and metastasis. Semin. Cancer Biol. 2000; 10; 415-433.

24. John A. Tuszynski G. The role of matrix metalloproteinases in tumor angiogenesis and tumor metastasis. Pathol. Oncol. Res. 2001; 7; 14-23.

25. Jiang Y, Goldberg ID, Shi YE. Complex roles of tissue inhibitors of metalloproteinases in cancer. Oncogene 2002: 21; 2245-2252.

26. Kugler A. Matrix metalloproteinases and their inhibitors. Anticancer Res. 1999; 19; 1589-1592.

27. Stetler-Stevenson WG. Matrix metalloproteinases in angiogenesis: a moving target for therapeutic intervention. J. Clin. Invest. 1999; 103; 1237-1241.

28. Basset P. Bellocq JP, Wolf $\mathrm{C}$ et al. A novel metalloproteinase gene specifically expressed in stromal cells of breast carcinomas. Nature 1990; 348 (6303); 699-704.

29. Polette M, Nawrocki B, Gilles C et al. MT-MMP expression and localisation in human lung and breast cancers. Virchows Arch. 1996: 428; 29-35.

30. Nelson AR, Fingleton B, Rothenberg ML, Matrisian LM. Matrix metalloproteinases: biologic activity and clinical implications. J. Clin. Oncol. 2000; 18; 1135-1149.

31. Still K, Robson CN, Autzen P. Robinson MC, Hamdy FC. Localization and quantification of mRNA for matrix metalloproteinase-2 (MMP-2) and tissue inhibitor of matrix metalloproteinase-2 (TIMP-2) in human benign and malignant prostatic tissue. Prostate 2000; 42; 18-25.

32. Yu Q. Stamenkovic I. Cell surface-localized matrix metalloproteinase- 9 proteolytically activates TGF-beta and promotes tumor invasion and angiogenesis. Genes Dev. 2000; 14; 163176.

33. Olson MW, Toth M. Gervasi DC, Sado X, Ninomiya Y, Fridman R. High affinity binding of latent matrix metalloproteinase-9 to the alpha2(IV) chain of collagen IV. J. Biol. Chem. 1998; 273; 10672-10681.

34. Barmina OY, Walling HW. Fiacco GJ et al. Collagenase- 3 binds to a specific receptor and requires the low density lipoprotein receptor-related protein for internalization. ]. Biol. Chem. 1999: 274; 30087-30093.

35. Shiraga M, Yano S. Yamamoto A et al. Organ heterogeneity of host-derived matrix metalloproteinase expression and its involve- ment in multiple-organ metastasis by lung cancer cell lines. Cancer Res. 2002; 62; 5967-5973.

36. Matrisian LM. Matrix metalloproteinase gene expression. Ann. NY Acad. Sci. 1994; 732; 42-50.

37. Xu X, Wang Y. Chen Z. Sternlicht MD, Hidalgo M, Steffensen B. Matrix metalloproteinase- 2 contributes to cancer cell migration on collagen. Cancer Res. 2005; 65; 130-136.

38. Misugi F, Sumi T, Okamoto $\mathrm{E}$ et al. Expression of matrix metalloproteinases and tissue inhibitors of metalloproteinase in uterine endometrial carcinoma and a correlation between expression of matrix metalloproteinase-7 and prognosis. Int. J. Mol. Med. 2005; 16; 541-546.

39. Yabushita $\mathrm{H}$, Narumiya $\mathrm{H}$, Hiratake $\mathrm{K}$ et al. The association of transforming growth factor-beta 1 with myometrial invasion of endometrial carcinomas through effects on matrix metalloproteinase. J. Obstet. Gynaecol. Res. 2000: 26; 163-170.

40. Ueno H. Yamashita K, Azumano I, Inoue M. Okada Y. Enhanced production and activation of matrix metalloproteinase-7 (matrilysin) in human endometrial carcinomas. Int. J. Cancer 1999; 84; $470-477$.

41. Moser PL, Kieback DG, Hefler L, Tempfer C, Neunteufel W, Gitsch G. Immunohistochemical detection of matrix metalloproteinases (MMP) 1 and 2, and tissue inhibitor of metalloproteinase 2 (TIMP 2) in stage IB cervical cancer. Anticancer Res. 1999; 19; 43914393.

42. Witty JP, McDonnell S, Newell KJ et al. Modulation of matrilysin levels in colon carcinoma cell lines affects tumorigenicity in vivo. Cancer Res. 1994; 54; 4805-4812.

43. Hasegawa S, Koshikawa N. Momiyama N et al. Matrilysinspecific antisense oligonucleotide inhibits liver metastasis of human colon cancer cells in a nude mouse model. Int. J. Cancer 1998: 76; 812-816.

44. Nathke IS, Hinck L, Swedlow JR, Papkoff J, Nelson WJ. Defining interactions and distributions of cadherin and catenin complexes in polarized epithelial cells. J. Cell Biol. 1994: 125; 1341-1352.

45. Hinck L, Nathke IS, Papkoff J, Nelson WJ. Dynamics of cadherin/catenin complex formation: novel protein interactions and pathways of complex assembly. J. Cell Biol. 1994; 125; 1327-1340.

46. Jou TS, Stewart DB, Stappert J. Nelson WJ, Marrs JA. Genetic and biochemical dissection of protein linkages in the cadherincatenin complex. Proc. Natl Acad. Sci. USA 1995: 92; 50675071.

47. Deugnier MA, Faraldo MM, Rousselle P. Thiery JP. Glukhova MA. Cell-extracellular matrix interactions and EGF are important regulators of the basal mammary epithelial cell phenotype. J. Cell Sci. 1999; 112; 1035-1044.

48. Park DW. Ryu HS, Choi DS, Park YH, Chang KH, Min CK. Localization of matrix metalloproteinases on endometrial cancer cell invasion in vitro. Gynecol. Oncol. 2001; 82; 442449.

49. Cioppi F, Simi L, Luciani P et al. Expression of uteroglobin and matrix metalloproteinase-9 genes in endometrial cancer: relationship to estrogen and progesterone receptor status. Oncol. Rep. 2004; $11 ; 427-433$.

50. Moreno-Bueno G, Hardisson D, Sarrio D et al. Abnormalities of $\mathrm{E}-$ and P-cadherin and catenin (beta-, gamma-catenin, and p120ctn) expression in endometrial cancer and endometrial atypical hyperplasia. J. Pathol. 2003; 199; 471-478.

51. Segawa T, Shozu M. Murakami K et al. Aromatase expression in stromal cells of endometrioid endometrial cancer correlates with poor survival. Clin. Cancer Res. 2005; 11; 2188-2194. 
52. Moreno-Bueno G, Hardisson D, Prat J. Matias-Guiu J, Palacios J. Re. Scholten et al. Nuclear beta-catenin is a molecular featurc of type I endometrial carcinoma. J. Pathol. 2003; 201; 460-465. J. Pathol. 2004; 202; 511-512.

53. Moreno-Bueno G. Hardisson D, Sanchez C et al. Abnormalities of the $\mathrm{APC} /$ beta-catenin pathway in endometrial cancer. Oncogene 2002; 21; 7981-7990.
54. Crawford HC, Fingleton BM, Rudolph-Owen LA et al. The metalloproteinase matrilysin is a target of beta catenin transactivation in intestinal tumors. Oncogene 1999; 18; 2883-2891.

55. Mylona E, Kapranou A, Mavrommatis J, Markaki S, Keramopoulos A. Nakopoulou L. The multifunctional role of the immunohistochemical expression of MMP-7 in invasive breast cancer. APMIS 2005: 113; 246-255. 


\section{Appendix 2}

Individual results of P53 from whole sections (WS), tissue microarray (TMA) and compressed scores (POS NEG) for Chapter 3 TMA Validation.

\begin{tabular}{|c|c|c|c|c|c|c|c|c|}
\hline Number & Ws & \begin{tabular}{|l|} 
WS \\
POS \\
NEG \\
\end{tabular} & $\begin{array}{l}\text { TMA } \\
\text { POS } \\
\text { NEG }\end{array}$ & $\begin{array}{l}\text { SCORE } \\
1\end{array}$ & $\begin{array}{l}\text { SCORE } \\
2\end{array}$ & $\begin{array}{l}\text { SCORE } \\
3\end{array}$ & $\begin{array}{l}\text { SCORE } \\
4\end{array}$ & MISSING \\
\hline 1 & & & 2 & 0 & 0 & 3 & 4 & 1 \\
\hline 2 & 2 & 2 & 2 & 0 & 1 & 0 & 1 & 2 \\
\hline 3 & & & 2 & 0 & 0 & 0 & 4 & 0 \\
\hline 4 & 1 & 2 & 2 & 0 & 6 & 0 & 0 & 0 \\
\hline 5 & 3 & 2 & 2 & 0 & 0 & 0 & 2 & 0 \\
\hline 6 & 2 & 2 & 1 & 4 & 0 & 0 & 0 & 0 \\
\hline 7 & & & & 0 & 0 & 0 & 0 & 4 \\
\hline 8 & & & 2 & 0 & 2 & 1 & 0 & 1 \\
\hline 9 & & & 2 & 4 & 0 & 2 & 0 & 0 \\
\hline 10 & & & 2 & 0 & 0 & 0 & 6 & 0 \\
\hline 11 & 3 & 2 & 2 & 0 & 0 & 2 & 0 & 0 \\
\hline 12 & 2 & 2 & 2 & 3 & 2 & 0 & 0 & 1 \\
\hline 13 & 2 & 2 & 2 & 0 & 1 & 1 & 0 & 0 \\
\hline 14 & 3 & 2 & 2 & 0 & 0 & 0 & 3 & 1 \\
\hline 15 & 3 & 2 & 2 & 0 & 0 & 0 & 5 & 1 \\
\hline 16 & 3 & 2 & 2 & 0 & 0 & 0 & 2 & 0 \\
\hline 17 & 3 & 2 & 2 & 0 & 0 & 0 & 3 & 1 \\
\hline 18 & 3 & 2 & 2 & 0 & 0 & 0 & 8 & 1 \\
\hline 19 & 2 & 2 & 2 & 0 & 4 & 0 & 0 & 0 \\
\hline 20 & & & 2 & 0 & 0 & 0 & 4 & 0 \\
\hline 21 & & & 2 & 0 & 0 & 0 & 4 & 0 \\
\hline 22 & & & & & & & & \\
\hline 23 & & & 2 & 0 & 0 & 0 & 6 & 0 \\
\hline 24 & & & 2 & 0 & 0 & 0 & 4 & 0 \\
\hline 25 & & . & 2 & 1 & 1 & 0 & 0 & 2 \\
\hline 26 & & & 1 & 4 & 0 & 0 & 0 & 0 \\
\hline 27 & 3 & 2 & 2 & 0 & 0 & 8 & 0 & 0 \\
\hline 28 & 0 & 1 & 1 & 2 & 0 & 0 & 0 & 2 \\
\hline 29 & 2 & 2 & 1 & 4 & 0 & 0 & 0 & 0 \\
\hline 30 & & & 2 & 0 & 0 & 2 & 0 & 0 \\
\hline 31 & 3 & 2 & 2 & 0 & 0 & 0 & 4 & 2 \\
\hline 32 & 2 & 2 & 2 & 0 & 0 & 2 & 0 & 0 \\
\hline 33 & 3 & 2 & 2 & 0 & 0 & 3 & 2 & 1 \\
\hline 34 & 3 & 2 & 2 & 0 & 0 & 0 & 3 & 1 \\
\hline 35 & & & 2 & 0 & 1 & 2 & 0 & 1 \\
\hline 36 & & & & 0 & 0 & 0 & 0 & 2 \\
\hline 37 & 2 & 2 & 2 & 0 & 1 & 3 & 0 & 2 \\
\hline 38 & & & 2 & 0 & 2 & 0 & 0 & 2 \\
\hline 39 & & & 2 & 0 & 0 & 0 & 10 & 0 \\
\hline 40 & & & 2 & 0 & 0 & 0 & 3 & 1 \\
\hline 41 & 3 & 2 & 2 & 0 & 0 & 0 & 4 & 0 \\
\hline 42 & 3 & 2 & 2 & 0 & 0 & 0 & 4 & 0 \\
\hline 43 & 0 & 1 & 1 & 3 & 0 & 0 & 0 & 1 \\
\hline 44 & 3 & 2 & 2 & 0 & 0 & 0 & 4 & 0 \\
\hline
\end{tabular}




\begin{tabular}{|c|c|c|c|c|c|c|c|c|}
\hline Number & WS & $\begin{array}{l}\text { WS } \\
\text { POS } \\
\text { NEG }\end{array}$ & $\begin{array}{l}\text { TMA } \\
\text { POS } \\
\text { NEG }\end{array}$ & $\begin{array}{l}\text { SCORE } \\
1\end{array}$ & $\begin{array}{l}\text { SCORE } \\
2\end{array}$ & $\begin{array}{l}\text { SCORE } \\
3\end{array}$ & $\begin{array}{l}\text { SCORE } \\
4\end{array}$ & MISSING \\
\hline 45 & 2 & 2 & 2 & 0 & 2 & 0 & 0 & 2 \\
\hline 46 & 3 & 2 & 2 & 0 & 0 & 0 & 4 & 0 \\
\hline 47 & 3 & 2 & 2 & 0 & 0 & 0 & 2 & 2 \\
\hline 48 & 3 & 2 & 2 & 0 & 0 & 0 & 7 & 1 \\
\hline 49 & . & . & 1 & 2 & 0 & 0 & 0 & 0 \\
\hline 50 & 2 & 2 & 2 & 0 & 0 & 2 & 0 & 0 \\
\hline 51 & 2 & 2 & 1 & 2 & 0 & 0 & 0 & 0 \\
\hline 52 & 3 & 2 & 2 & 0 & 0 & 0 & 6 & 0 \\
\hline 53 & 3 & 2 & 2 & 0 & 0 & 0 & 1 & 1 \\
\hline 54 & 2 & 2 & & . & . & . & . & . \\
\hline 55 & . & . & . & . & . & . & . & $\dot{.}$ \\
\hline 56 & 2 & 2 & . & 0 & 0 & 0 & 0 & 2 \\
\hline 57 & 3 & 2 & 1 & 2 & 0 & 0 & 0 & 0 \\
\hline 58 & . & . & 2 & 0 & 1 & 1 & 0 & 0 \\
\hline 59 & 3 & 2 & 2 & 0 & 0 & 0 & 2 & 0 \\
\hline 60 & 3 & 2 & 2 & 0 & 0 & 0 & 4 & 0 \\
\hline 61 & 3 & 2 & 2 & 0 & 0 & 1 & 0 & 1 \\
\hline 62 & 2 & 2 & 1 & 2 & 0 & 0 & 0 & 2 \\
\hline 63 & 2 & 2 & 2 & 1 & 1 & 0 & 0 & 2 \\
\hline 64 & . & . & 2 & 0 & 6 & 0 & 0 & 0 \\
\hline 65 & . & . & 2 & 1 & 4 & 1 & 0 & 0 \\
\hline 66 & . & . & 2 & 0 & 0 & 1 & 2 & 1 \\
\hline 67 & . & . & 2 & 0 & 0 & 0 & 6 & 0 \\
\hline 68 & . & . & 2 & 0 & 0 & 0 & 3 & 1 \\
\hline 69 & . & . & 2 & 0 & 0 & 0 & 4 & 0 \\
\hline 70 & - & . & 2 & 0 & 0 & 0 & 3 & 1 \\
\hline 71 & & . & 1 & 2 & 0 & 0 & 0 & 0 \\
\hline 72 &. & . & 2 & 0 & 0 & 0 & 4 & 0 \\
\hline 73 & 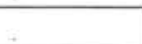 & 3 & 2 & 0 & 0 & 1 & 6 & 1 \\
\hline 74 &. & . & 2 & 0 & 0 & 0 & 2 & 2 \\
\hline 75 & . & . & 2 & 0 & 3 & 1 & 0 & 0 \\
\hline 76 & . & & 1 & 5 & 0 & 0 & ก & 1 \\
\hline 77 & . & & 2 & 0 & 0 & 2 & 2 & 0 \\
\hline 78 & . & . & 2 & 0 & 5 & 1 & 0 & 0 \\
\hline 70 & & & 1 & 2 & 0 & 0 & 0 & 2 \\
\hline 80 & 3 & 2 & & U & U & U & U & 2 \\
\hline
\end{tabular}




\section{Appondix 3}

Individual results of Oestrogen receptor status (ER) from whole sections (WS), tissue

Validation.

\begin{tabular}{|c|c|c|c|c|}
\hline Case number & WS total & TMA total & WS POS NEG & TMA POS NEG \\
\hline 1 & - & 2.63 & & 1 \\
\hline$?$ & 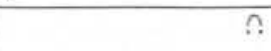 & & 4 & \\
\hline 3 & & 4.5 &. & 2 \\
\hline 4 & 4 & 2.17 & 1 & 1 \\
\hline 5 & 6 & 6 & 2 & 2 \\
\hline 6 & 8 & 5.25 & 2 & 2 \\
\hline 7 & . & & . & $\dot{.}$ \\
\hline 8 & . & 6 & . & 2 \\
\hline 3 & . & 0.00 & . & 2 \\
\hline 10 & . & 3.5 & . & 1 \\
\hline 11 & 5 & 6 & 2 & 2 \\
\hline$i_{2}$ & 5 & 2.5 & 2 & : \\
\hline 13 & 4 & 0 & 1 & 1 \\
\hline 14 & 0 & 0.5 & 1 & 1 \\
\hline$\therefore=$ & 7 & $=$ & $\because$ & $\because$ \\
\hline 16 & 4 & 0 & 1 & 1 \\
\hline 17 & 4 & 3.33 & 1 & 1 \\
\hline 49 & $\therefore$ & 1 & 4 & 1 \\
\hline 19 & 4 & 2 & 1 & 1 \\
\hline 20 & . & 4 & . & 1 \\
\hline 21 & . & 3 & . & 1 \\
\hline 22 & . & & . & . \\
\hline 23 & . & 4.23 & - & 2 \\
\hline 24 & 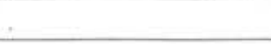 & 8 & . & 2 \\
\hline 25 & . & 0 & . & 2 \\
\hline 26 & . & 5.75 & . & 2 \\
\hline 27 & 0 & 0 & 1 & 1 \\
\hline $2 \overline{5}$ & $\bar{Q}$ & & $\vdots$ & \\
\hline 29 & 0 & 0 & 1 & 1 \\
\hline 30 & . & 6 & . & 2 \\
\hline 34 & 5 & 775 & 3 & 3 \\
\hline 32 & 5 & 5.5 & 2 & 2 \\
\hline 33 & 6 & 6.8 & 2 & 2 \\
\hline$?: 1$ & $\therefore$ & $\therefore$ & 4 & 4 \\
\hline 35 & & 7 & & 2 \\
\hline 36 & . & & & \\
\hline 37 & 4 & 1.25 & 1 & 1 \\
\hline 38 &. & 4.5 & . & 2 \\
\hline 39 & . & 6.6 & . & 2 \\
\hline 40 & & 5.33 & & 2 \\
\hline $4 i$ & 0 & 7.25 & 2 & 2 \\
\hline 42 & 6 & 0.5 & 2 & 1 \\
\hline 43 & 3 & 3.33 & 1 & 1 \\
\hline 45 & 3 & & & $\vdots$ \\
\hline
\end{tabular}




\begin{tabular}{|c|c|c|c|c|}
\hline Case number & WS total & TMA total & WS POS NEG & TMA POS NEG \\
\hline 45 & 8 & 8 & 2 & 2 \\
\hline$\Delta \therefore$ & ? & $\therefore ?$ & $?$ & 2 \\
\hline 47 & 4 & 2.5 & 1 & 1 \\
\hline 48 & 7 & 6.14 & 2 & 2 \\
\hline 49 & & 7 & & 2 \\
\hline 50 & 6 & 5.5 & 2 & 2 \\
\hline 51 & 5 & 2.5 & 2 & 1 \\
\hline 52 & 6 & 2.37 & 2 & 1 \\
\hline 50 & 0 & 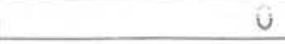 & 2 & i \\
\hline 54 & 7 & & 2 & \\
\hline 55 & . & . & . & . \\
\hline 55 & 4 & & $\mathrm{a}$ & $\therefore$ \\
\hline 57 & 0 & 0 & 1 & 1 \\
\hline 58 & . & 3 & . & 1 \\
\hline$\because$ & I & & $\because$ & \\
\hline 60 & 6 & 7 & 2 & 2 \\
\hline 61 & 3 & . & 1 & . \\
\hline$\therefore 2$ & 7 & 5 & 2 & 2 \\
\hline 63 & 6 & 6.33 & 2 & 2 \\
\hline 64 & . & 7.83 & . & 2 \\
\hline 65 & 4 & 3.2 & 1 & 1 \\
\hline 66 & . & - & . & . \\
\hline 67 & . & 7 & . & 2 \\
\hline 68 & . & 5 & - & 2 \\
\hline 00 & , & $u$ & . & $i$ \\
\hline 70 & . & 5 & & 2 \\
\hline 71 & . & 3 & & 1 \\
\hline 72 & . & 5.33 & & 2 \\
\hline 73 &. & 3.13 & & 1 \\
\hline 74 & . & 5 & & 2 \\
\hline 75 & & $7 \%$ & & $\because$ \\
\hline 76 & . & 6.25 & & 2 \\
\hline 77 & . & 6 & . & 2 \\
\hline 73 & & $7 \%$ & & $?$ \\
\hline 79 & . & 7 & . & 2 \\
\hline 80 & 5 & & 2 & \\
\hline
\end{tabular}

